\title{
Identification and Classification of Radio Pulsar Signals Using Machine Learning
}

DI Pang

West Virginia University, dipang@mix.wvu.edu

Follow this and additional works at: https://researchrepository.wvu.edu/etd

Part of the Artificial Intelligence and Robotics Commons, Data Science Commons, and the Other Astrophysics and Astronomy Commons

\section{Recommended Citation}

Pang, DI, "Identification and Classification of Radio Pulsar Signals Using Machine Learning" (2021). Graduate Theses, Dissertations, and Problem Reports. 10280.

https://researchrepository.wvu.edu/etd/10280

This Dissertation is protected by copyright and/or related rights. It has been brought to you by the The Research Repository @ WVU with permission from the rights-holder(s). You are free to use this Dissertation in any way that is permitted by the copyright and related rights legislation that applies to your use. For other uses you must obtain permission from the rights-holder(s) directly, unless additional rights are indicated by a Creative Commons license in the record and/ or on the work itself. This Dissertation has been accepted for inclusion in WVU Graduate Theses, Dissertations, and Problem Reports collection by an authorized administrator of The Research Repository @ WVU.

For more information, please contact researchrepository@mail.wvu.edu. 


\title{
Identification and Classification of Radio Pulsar Signals Using Machine Learning
}

\author{
Di Pang \\ Dissertation submitted to the \\ Benjamin M. Statler College of Engineering and Mineral Resources \\ at West Virginia University \\ in partial fulfillment of the requirements \\ for the degree of \\ Doctor of Philosophy \\ in \\ Computer Science
}

Katerina Goseva-Popstojanova, Ph.D., Committee Chairperson Xin Li, Ph.D.

Duncan Lorimer, Ph.D. Maura McLaughlin, Ph.D.

Nasser Nasrabadi, Ph.D.

Natalia Schmid, Ph.D.

Lane Department of Computer Science and Electrical Engineering

\author{
Morgantown, West Virginia
}

2021

Keywords: machine learning, clustering, classification, class imbalance, rare events, recommender system, pulsar, radio astronomy 


\section{Abstract}

\section{Identification and Classification of Radio Pulsar Signals Using Machine Learning \\ Di Pang}

Automated single-pulse search approaches are necessary as ever-increasing amount of observed data makes the manual inspection impractical. Detecting radio pulsars using single-pulse searches, however, is a challenging problem for machine learning because pulsar signals often vary significantly in brightness, width, and shape and are only detected in a small fraction of observed data.

The research work presented in this dissertation is focused on development of machine learning algorithms and approaches for single-pulse searches in the time domain. Specifically, (1) We developed a two-stage single-pulse search approach, named SinglePulse Event Group IDentification (SPEGID), which automatically identifies and classifies pulsars in radio pulsar search data. SPEGID first identifies pulse candidates as trial single-pulse event groups and then extracts features from the candidates and trains classifiers using supervised machine learning. SPEGID also addressed the challenges introduced by the current data processing techniques and successfully identified bright and dim candidates as well as other types of challenging pulsar candidates. (2) To address the lack of training data in the early stages of pulsar surveys, we explored the cross-surveys prediction. Our results showed that using instance-based and parameter-based transfer learning methods improved the performance of pulsar classification across surveys. (3) We developed a hybrid recommender system aimed to detect rare pulsar signals that are often missed by supervised learning. The proposed recommender system uses a target rare case to state users' requirements and ranks the candidates using a similarity function which is calculated as a weighted sum of individual feature similarities. Our hybrid recommender system successfully detects both low signal-to-noise ratio $(\mathrm{S} / \mathrm{N})$ pulsars and Fast Radio Bursts (FRBs).

The approaches proposed in this dissertation were used to analyze data from the Green Bank Telescope $350 \mathrm{MHz}$ drift (GBTDrift) pulsar survey and the Arecibo 327 $\mathrm{MHz}$ (AO327) drift pulsar survey and discovered eight pulsars that were overlooked in previous analysis done with existing methods. 


\section{Acknowledgments}

This work was in part supported by NSF award \#1458952. The completion of my dissertation would not have been possible without the financial support.

I would like to express my deepest appreciation to my committee chair and advisor, Dr. Katerina Goseva-Popstojanova for offering me this great opportunity to work with her. During my Ph.D. study, she not only trained me with great patience to become a research scientist, but always gave me guidance and advice when I needed them. I'm also grateful for her strong support of my own ideas and research directions, and her confidence in my abilities. I would like to extend my deepest gratitude to Dr. Maura McLaughlin. Not only did she spent much time answering my questions and providing comments to my papers, more importantly, she has always provided me with encouragement and patience throughout the duration of this project. I would also like to thank many members of the astronomical community, including Drs. Duncan Lorimer, Sarah Burke-Spolaor, Julia Deneva, WVU Pulsar Research Group, and many others. Their willingness to share with their knowledge helped me learn more about pulsar astronomy and make progresses in my research. Moreover, many thanks to the PALFA collaboration for making data available and for helpful discussions.

I would also like to thank my committee members from the WVU Statler College. They always respond to my emails promptly and their passion and devotion have been a source of inspiration to me. I also had great pleasure of working with my lab-mates, including Thomas Devine, Thomas Kyanko, Mohammad J. Ahmad, Denny Hood, Lingwei Chen, etc. Special thanks to Thomas Devine for teaching me about machine learning and Weka when I started working on this project.

Finally, I would like to thank my friends and family especially my brother Yue Pang for their love, support and constant encouragement. 


\section{Contents}

Acknowledgments $\quad$ iii

List of Figures vii

List of Tables xiv

List of Acronyms xii

1 Introduction 1

1.1 Motivation .......................... 1

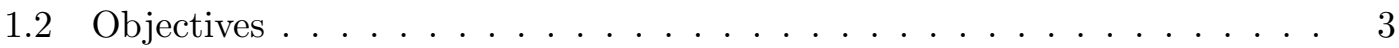

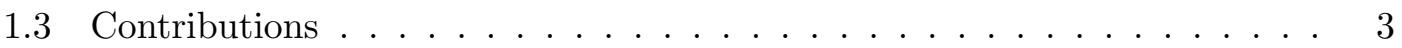

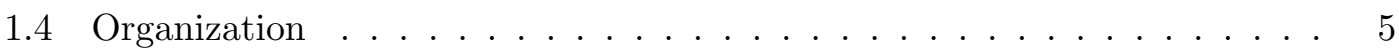

2 Background $\quad 6$

2.1 Pulsar Astronomy Background _. . . . . . . . . . . . . . . 6

2.2 Pulsar Searches Background . . . . . . . . . . . . . . . . 7

2.2.1 Pulsar Search Pipeline . . . . . . . . . . . . . . . 8

2.2.2 Pulsar Search Collaboratory . . . . . . . . . . . . . . . . 10

2.2.3 Radio Pulsar Data Sets . . . . . . . . . . . . . . . . . . . 13

2.3 Machine Learning Background . . . . . . . . . . . . . . . . . 13

2.3.1 Definition of Machine Learning . . . . . . . . . . . . . . . 13

2.3.2 Supervised Machine Learning Algorithms . . . . . . . . . . . . . . 14

2.3.3 Unsupervised Machine Learning Algorithms . . . . . . . . . . . . . 20

2.3.4 Transfer Learning . . . . . . . . . . . . . . . . . . . . 21

2.3.5 Imbalanced Learning . . . . . . . . . . . . . . . . . . . . . . 21

2.3.6 Recommender Systems . . . . . . . . . . . . . . . . . . 23

2.3 .7 Performance Metrics . . . . . . . . . . . . . . . . . . . . 24

2.3.8 Performance Metrics for the Evaluation of Classifications . . . . . 24

2.3.9 Performance Metrics for Evaluation of Recommender Systems . . . 26

3 Related Work $\quad 29$

3.1 Related Work on Periodicity Searches . . . . . . . . . . . . . . . 29

3.2 Related Work on Single-pulse Searches . . . . . . . . . . . . . . . . . . . 30

3.3 Related Work on Transfer Learning . . . . . . . . . . . . . . . . . . . . . . 33

3.4 Related Work on Mining with Rarity . . . . . . . . . . . . . . . . . . . . . 34 
4 SPEGID: Identification and Classification of Pulsar Signals $\quad 37$

4.1 Approach Outline . . . . . . . . . . . . . . . . . 37

4.2 Single-pulse Events . . . . . . . . . . . . . . . . . . . . . . . . 40

4.3 Identification of SPEGs . . . . . . . . . . . . . . . . . . . 42

4.3.1 DBSCAN Clustering . . . . . . . . . . . . . . . 42

4.3.2 Merging Clusters Vertically into SPEGs . . . . . . . . . . . . 47

4.3.3 Calculating the Peak Score for SPEGs . . . . . . . . . . . . . . . 53

4.3.4 Grouping SPEGs Horizontally . . . . . . . . . . . . . . . . 63

4.4 Assessment of the Effectiveness of SPEGID . . . . . . . . . . . . . . 65

4.5 Automatic Classification of SPEGs Using Supervised Machine Learning . 66

4.5.1 Feature Extraction . . . . . . . . . . . . . . . . . . 67 67

4.5.2 Creating the Benchmark Data Set . . . . . . . . . . . . . 67

4.5.3 Tested Machine Learning Algorithms . . . . . . . . . . . . . . . . . 69

4.5.4 Imbalance Consideration . . . . . . . . . . . . . . . . . . 69

4.5.5 Metrics for Evaluation of the Classifications . . . . . . . . . . . 70

4.5.6 Automatic Classification of the Unlabeled Full Data Set . . . . . . 71

4.6 Checking Frequency-time Signatures for Bright SPEGs . . . . . . . . . . . 71

4.7 Searching for Periodicity among Dim SPEGs . . . . . . . . . . . . 71

4.8 Results . . . . . . . . . . . . . . . . . . . . . 75

4.8.1 Results Based on the Benchmark Data Set by SPEG . . . . . . . . 77

4.8.2 Results Based on the Benchmark Data Set by Beam . . . . . . . . 78

4.8.3 Results Based on the Full Data Set . . . . . . . . . . . . . . . 80

4.9 Discovery of a New Pulsar from GBTDrift Survey . . . . . . . . . . . . . 84

4.10 Discussion of the Challenges and Implications . . . . . . . . . . . . . . . 84

4.11 Conclusion . . . . . . . . . . . . . . . . . . 88

5 Pulsar Classification Using Boosting and Deep Learning 89

5.1 Feature Extraction Extended . . . . . . . . . . . . . . . . 89

5.2 Creating Two Benchmark Data Sets . . . . . . . . . . . . . . . 90

5.3 SPEG Classification Using AdaBoost . . . . . . . . . . . . . . . . . . . 92

5.4 SPEG Classification Using Deep Learning . . . . . . . . . . . . . . . . . . 92

5.5 Hyperparameters Tuning . . . . . . . . . . . . . . . . . . . . . . 93

5.6 Performance Metrics . . . . . . . . . . . . . . . . . . . . . 93

5.6.1 Binary Classification versus Probabilistic Prediction . . . . . . . 94

5.7 Classification of SPEGs versus Pulsars . . . . . . . . . . . . . . . 95

5.8 Experimental Results . . . . . . . . . . . . . . . . . . . . 95

5.8.1 GBTDrift Benchmark Classification . . . . . . . . . . . . . 96

5.8.2 PALFA-extended Benchmark Classification . . . . . . . . . . . 100

5.9 Conclusion . . . . . . . . . . . . . . . . . . . . . . . . 104

6 Discovery of Seven Pulsars from the AO327 Survey Using SPEGID 105

6.1 AO327 Drift Pulsar Survey Description _ . . . . . . . . . . . . . 105

6.2 Clusterrank . . . . . . . . . . . . . . . . . . 106

6.2 .1 Clusterrank versus SPEGID . . . . . . . . . . . . 106

6.3 Results . . . . . . . . . . . . . . . . . . . . . 107

6.4 Conclusion . . . . . . . . . . . . . . . . . . . 108 
$\begin{array}{lll}7 & \text { Pulsar Classification Across Surveys } & 117\end{array}$

7.1 Cross-survey Prediction without Transfer Learning . . . . . . . . . . . . . 118

7.1.1 Classifying GBTDrift Using Models Trained on PALFA-extended . 118

7.1.2 Classifying PALFA-extended Using Models Trained on GBTDrift . 119

7.2 Training on a Combined Data Set without Transfer Learning . . . . . . . 119

7.2.1 Classifying GBTDrift Using Models Trained on a Combined Data

Set . . . . . . . . . . . . . . . . 120

7.2.2 Classifying PALFA-extended Using Models Trained on a Combined Data Set . . . . . . . . . . . . . . . . . . 121

7.3 Cross-survey Results with Transfer Learning . . . . . . . . . . . . . . . . . 121

7.3.1 trAdaBoost . . . . . . . . . . . . . . . . . 122

7.3 .2 Fine-tuning SPEGnet . . . . . . . . . . . . . . . . . 122

7.3.3 Hyperparameter Tuning . . . . . . . . . . . . . . . . . . . 122

7.3.4 Classifying GBTDrift Using Transfer Learning . . . . . . . . . . . 123

7.3.5 Classifying PALFA-extended Using Transfer Learning . . . . . . . 124

7.4 Summary of the Results and Discussion . . . . . . . . . . . . . . 125

7.5 Conclusion . . . . . . . . . . . . . . . . 130

8 Detection of Rare Signals Using a Novel Hybrid Recommender System131

8.1 Example of Rare Cases in Pulsar Searches . . . . . . . . . . . . . . . . . 132

8.2 Our Proposed Hybrid Recommender Systems . . . . . . . . . . . . . . . . 134

8.2.1 Similarity Metric Between Feature Values . . . . . . . . . . . . 136

8.2.2 Weighting of Features in our Hybrid Recommender System . . . . 138

8.2 .3 Calculation of Dependence Coefficient . . . . . . . . . . . . . 138

8.2.4 Improving User's Interest Inference Using an Outlier Score . . . . 140

8.3 Experiments . . . . . . . . . . . . . . . . . . . . . . . 141

8.4 Results . . . . . . . . . . . . . . . . . . . . . . . . 142

8.4.1 Detection of Low S/N Pulsars . . . . . . . . . . . . . . . 142

8.4 .2 Detection of FRBs . . . . . . . . . . . . . . . . . 144

8.5 Conclusion . . . . . . . . . . . . . . . . . 146

9 Conclusion and Future Work 148

9.1 Look-back . . . . . . . . . . . . . . . . . . . . . . . . . . . . . . . 148

9.2 Main Contributions . . . . . . . . . . . . . . . . . . . . . . 149

9.3 Future Work . . . . . . . . . . . . . . . . . . . . . . . 150

$\begin{array}{ll}\text { List of Publications } & 151\end{array}$

Bibliography 152 


\section{List of Figures}

2.1 As a pulsar rotate, beams of radiation are emitted at its magnetic poles. Credit: B. Saxton, NRAO/AUI/NSF . . . . . . . . . . . . . . 7

2.2 Diagram of a radio telescope. Credit: http://abyss.uoregon.edu/ js/ glossary/radio_telescope.html (accessed in November 2021). . . . . .

2.3 A graphical representation of a filterbank file. The top panel represents the time series and shows the observed power as a function of time. A filterbank is an array of bandpass filters that separates the input signal into multiple narrow frequency channels, which is shown as the frequency versus time subplot in the middle panel. The bandpass in shown in right panel and it demonstrates the observed power as a function of frequency. In other words, the time series shows the total power in all frequencies and the bandpass shows the power added over all times. Credit: [60] . .

2.4 PRESTO-based single-pulse search pipeline. The outline of PRESTO search shows how the time series date are processed. The top subplot shows the dispersion of pulsar signals due to its interaction with ISM. The bottom panel shows in details how single-pulse search files are created and an example of single-pulse search diagnostic plot. . . . . . . . . . . . .

2.5 Diagnostic plot of signals from a periodic pulsar. This plots including subplots of the time series, pulse profile, the signal strength as a function of phase and observing frequency, the reduced $\chi^{2}$ versus DM, the reduced $\chi^{2}$ versus Period, the reduced $\chi^{2}$ versus P-dot, and the P-dot versus Period. 11

2.6 Known pulsar J1901+0355 detected on MJD 56245 in the PALFA survey. The four subplots include (clockwise from top left): a histogram of the number of single-pulse events versus $\mathrm{S} / \mathrm{N}$, a histogram of the number of single-pulse events versus DM, a scatter plot of $\mathrm{S} / \mathrm{N}$ versus $\mathrm{DM}$ and a scatter plot of the DM versus time for each single-pulse event whose size

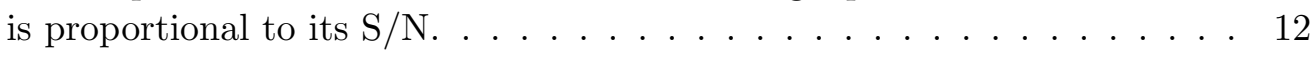

2.7 Decision tree for the weather data set. . . . . . . . . . . . . 16

2.8 Schematic of $2 \mathrm{D}$ convolution. . . . . . . . . . . . . . . . . 19

2.9 Comparision between image classification, objection detection and instance segmentation. Credit: [76]. . . . . . . . . . . . . 20

2.10 Graphical representation of rare class and rare cases. . . . . . . . . . . . 22

2.11 Examples of ROC curves. Each curve represents the performance of a different classifier on the given data set. Credit: [96] . . . . . . . . . . 26

4.1 The outline of our proposed approach SPEGID . . . . . . . . . . . . 38 
4.2 A typical astrophysical pulse detected as of a group of single-pulse events. The bottom and the top left subplots show its appearance in the DM versus time space, and the top right subplot show its $\mathrm{S} / \mathrm{N}$ versus $\mathrm{DM}$ curve. Note that in both the top left and the bottom subplots, the singlepulse events are both plotted proportionally to $\mathrm{S} / \mathrm{N}$ but with different scaling to show the single-pulse events more clearly in the top left subplot. In the top right subplot, we also show both the fitted and expected $\mathrm{S} / \mathrm{N}$ decline that are caused by DM offset. Note that they are both fairly close to the observed $\mathrm{S} / \mathrm{N}$ decline, and to each other as well. . . . . . . . . .

4.3 A zoomed-in part of the diagnostic plot shown in Figure 2.6. The region surrounded by the solid rectangle in the bottom subplot is further enlarged to show the details in the top left subplot, in which three clusters of singlepulse events can be seen. The smaller cluster on the right (surrounded by a dashed rectangle) is zoomed in and shown in the top middle and the top right subplots. This cluster is used to demonstrate DBSCAN clustering in Figure $4.4 \ldots \ldots \ldots \ldots \ldots \ldots$

4.4 An example of DBSCAN clustering applied on the cluster from Figure 4.3. Note that instead of the actual time and DM, time and DM channel indices were used to calculate the distance between single-pulse events. In this example, all single-pulse events are core objects in DBSCAN clustering and the radius of their neighborhood is small enough to avoid including any noise event. Because their neighborhood areas overlap with one another, all single-pulse events are joined together to form one single cluster. It can also be seen for two adjacent single-pulse events, a unit increase in DM channel index roughly corresponds to a unit decrease of time index.

4.5 Known pulsar J1910+0714 detected on MJD 56663 in the PALFA survey. Note that there are many clipped pulses. The top left subplot and the bottom subplot show the $\mathrm{S} / \mathrm{N}$ versus DM space, and the DM versus time space of this whole section respectively; and the other two subplots show those regions around the clipped pulse of interest that is surrounded by the red dashed line rectangle. . . . . . . . . . . . . . . . .

4.6 Merging the clusters in a non-clipped pulse. In the left and middle subplots, the single-pulse events are both plotted proportionally to $\mathrm{S} / \mathrm{N}$ but with different scaling in order to show the distance between single-pulse events more clearly. In the middle subplot, the red circle shows the brightest single-pulse event, and the red solid rectangle shows the expected DM and time span of the pulse. In the right subplot, the red circle shows the brightest single-pulse event and the red dashed line shows its DM, and the blue dashed line shows the expected (i.e., not fitted) $\mathrm{S} / \mathrm{N}$ decline. The black horizontal dashed segment shows the $\mathrm{S} / \mathrm{N}$ threshold $\left(\mathrm{S}_{\text {th }}\right)$ calculated from Equation (4.4). The two red vertical segments show the limit of the $\mathrm{DM}$ offset where the $\mathrm{S} / \mathrm{N}$ decreases below $\mathrm{S}_{\mathrm{th}}$. The arrows show where the pulse breaks into several clusters in DBSCAN clustering because a small radius is used. . . . . . . . . . . . . . . . . . . . 
4.7 Merging the clipped pulse from Figure 4.5. The red rectangle in the middle subplot shows the calculated DM and time ranges. Compared with the left subplot, the single-pulse events in the middle subplot are plotted using a smaller scaling to show more details. In the middle and right subplots, the red circle shows the single-pulse event with the maximum $\mathrm{S} / \mathrm{N}$ among all clusters returned by DBSCAN clustering, from which the calculated DM and time span of the pulse is calculated and shown as the red solid rectangle in the middle subplot. Note that the hypothetical peak event is not the brightest event in the right subplot as all the brighter events are excluded from DBSCAN clustering (i.e., there are less than MinPts events in their $\epsilon$ neighborhood, and they don't fall into the $\epsilon$ neighborhood of any other core point) and are classified as "noise" (they are not really noise events). In the right subplot, the blue dashed line is the expected S/N decline calculated from the hypothetical peak. The two black horizontal dashed segments show the $\mathrm{S} / \mathrm{N}$ threshold $\left(\mathrm{S}_{\text {th }}\right)$ calculated from Equation (4.5). The two red vertical segments shows the limit of the $\mathrm{DM}$ offset where the $\mathrm{S} / \mathrm{N}$ decreases below $\mathrm{S}_{\mathrm{th}}$. By using different $\mathrm{S}_{\mathrm{th}}$ obtained on the two sides, the expected DM and time span is closer to the actual span. . . . . . . . . . . . . . . . . .

4.8 The DM versus time plot for pulsar J1901+02 detected in the PALFA survey. The top subplot shows all single-pulse events within the DM and time range, while the bottom subplot only shows those single-pulse events that belong to SPEGs identified by DBSCAN clustering and merging of clusters. The comparison between the top and bottom subplots illustrates the denoising effect of these two steps. . . . . . . . . . . . . .

4.9 The application of OLS linear regression and WLS linear regression on a clipped pulse. The top left subplot shows the SPEG in the DM versus time space, while the other three plots show the $\mathrm{S} / \mathrm{N}$ versus $\mathrm{DM}$ curve. While the top right subplot shows all single-pulse events within the SPEG, the bottom two plots only keep the brightest event if there is more than one event at the same DM. In the bottom left subplot, the fit lines were obtained through OLS liner regression, and the second fit line is strongly influenced by those dim events, whereas in the bottom right subplot, their influence is significantly reduced by WLS linear regression. The intersection of the middle two fit lines is recorded as the center DM (center $D M$ ), and its value is shown by the black dashed line. It can be seen that center DM obtained from WLS linear regression is much closer to the $\mathrm{DM}$ of the clipped peak. . . . . . . . . . . . . . .

4.10 An example of a wide, not very bright pulse. Given the $\mathrm{DM}$ spacing at the
peak $\mathrm{S} / \mathrm{N}$ is $0.3 \mathrm{pc}^{-1} \mathrm{~cm}^{3}, \mathrm{SL}_{\text {th }}$ calculated by equations (4.8) and (4.9) is 0.03 , and the peak score is 6 . The slopes of the six fit lines calculated from WLS linear regression are (from left to right): $0.140,0.283,0.078,-0.134$, -0.056 and $-0.038 \mathrm{pc}^{-1} \mathrm{~cm}^{3}$. Thus using any slope threshold greater than 0.14 will result in a peak score less than 2 , and cause this pulse to be overlooked. . . . . . . . . . . . . . . . . 58 
4.11 Calculating the peak score of a regular pulse from Figure 4.6. While the left subplot shows all single-pulse events within the SPEG, the right subplot shows only the brightest event if there is more than one event at the same DM. Only these events are used in finding the peak score. The calculated peak score for this pulse is $6 \ldots \ldots \ldots \ldots$

4.12 Calculating the peak score of a clipped pulse from Figure 4.7. While the left subplot shows all events within the SPEG, the right subplot shows only the brightest event if there is more than one event at the same DM. Only these events are used in finding the peak score. The calculated peak score for this pulse is $4 \ldots \ldots \ldots \ldots \ldots \ldots$

4.13 The DM versus time plot for pulsar J1901+02. The top subplot shows all SPEGs with a peak score $\geq 2$. By filtering SPEGs with a peak score $<2$, the number of SPEGs is decreased from 81 (shown in the bottom subplot of Figure 4.8) to 48. The bottom subplot only shows those bright SPEGs with a peak score $\geq 2$ and with a peak $\mathrm{S} / \mathrm{N} \geq 6$ (see Section 4.3.1 for the definition of bright SPEGs). These SPEGs are used to form new SPEG groups in Section 4.3.4. . . . . . . . . . . . . . .

4.14 Examples of astrophysical pulses with different $\mathrm{S} / \mathrm{N}$ versus DM curve shapes (a, c, e, g), and their calculated peak scores (b, d, f, h). These pulses are from the detection of the known pulsar J2003+29 (shown in Figure 4.15) in the PALFA survey. The authenticity of these pulses is confirmed by searching for the underlying periodicity among them and comparing it with the known period of the pulsar (see Section 4.7 for details). The $\mathrm{S} / \mathrm{N}$ versus DM curve in Figure 4.14(g) looks different than expected, presumably due to the weakness of the pulse. . . . . . . . .

4.15 Known pulsar J2003+29 detected on MJD 57039 in the PALFA survey. The three subplots in the top include a histogram of the number of singlepulse events versus $\mathrm{S} / \mathrm{N}$, a histogram of the number of single-pulse events versus DM, a scatter plot of $\mathrm{S} / \mathrm{N}$ versus $\mathrm{DM}$. The middle subplot is a scatter plot of the DM versus time for each single-pulse event where its sise is proportional to its $\mathrm{S} / \mathrm{N}$. The bottom subplot shows only SPEGs with peaks identified. The numbers 1,4 and 52 are the ranks of the SPEGs by their peak $\mathrm{S} / \mathrm{N}$ in descending order. . . . . . . . . . . . . .

$4.16 \mathrm{~S} / \mathrm{N}$ versus DM plots of numbered SPEGs 1, 4 and 52 from Fig 4.15. The shaded area in each plot shows the central part of the SPEG. In the bottom right plot, the central part of SPEG 1 is plotted as a transparent region (the two black solid lines represent its DM range) to show its overlap with that of SPEG 3 more clearly. Note that although the DMs of the peak events differ, the central parts of SPEGs 4 and 52 overlap with that of SPEG 1 and the DM of the peak event of SPEG 1 falls into the full DM ranges of SPEGs 4 and 52. Therefore the three SPEGs are grouped together. 64

4.17 Grouped SPEGs from Figure 4.15. Different colours represent different SPEG groups. The number in parentheses is the group number of each SPEG group. For example, SPEG group 1 contains 17 SPEGs (including SPEGs $1,4,52$, etc.) plotted in black, and SPEG group 2 contains only 1 SPEG (SPEG 15) plotted in red, etc. Each group has at least one bright SPEG, with a peak $\mathrm{S} / \mathrm{N} \geq 6$. 
4.18 Confusion matrix for SPEG classification. . . . . . . . . . . . . . . 70

4.19 Known pulsar J1910+0728 detected on MJD 56663 in the PALFA survey. The bottom subplot shows only SPEGs that have peak score $\geq 2$ and belong to an SPEG group containing at least one SPEG with peak S/N $\geq 6$. The red pulses are astrophysical pulses with an underlying period of $0.325 \mathrm{~s}$, which agrees with the period of this pulsar. . . . . . . . .

4.20 The pulse profile (top) and dynamic spectrum (bottom) of the pulse detected at $9.26 \mathrm{~s}$ in Figure 4.19 with maximum $\mathrm{S} / \mathrm{N}=8.36$, dedispersed to $\mathrm{DM}=283.9 \mathrm{pc} \mathrm{cm}-3$ and summed to 32 frequency channels across the band. The total time plotted is $300 \mathrm{~ms}$. . . . . . . . . . . . . . . 73

4.21 Detection of known pulsar J1935+2025 on MJD 56431 in the PALFA

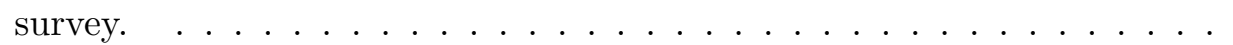

4.22 A Venn diagram showing the results of automatic classification and manual inspection of beams that contained positively classified SPEG(s). Among these 47,042 beams, only 1,299 beams contained at least one positively classified SPEG. Through quick-look manual inspection of these 1,299 beams, we found all 90 known (60 distinct) pulsars included in the benchmark data set correctly classified (CKs), 84 additional known pulsars not included in the benchmark data set (AKs), 1031 false positives (FPs), i.e., non-pulsars incorrectly classified as pulsars), 89 unlikely candidates (UCs), and 5 possible discoveries (PDs). . . . . . . . . .

4.23 One possible discovery. The five SPEGs plotted in red in the bottom subplot are "periodic" at the tolerance level of 0.03. The probability of finding periodicity among five random events at such tolerance is 0.043 . Nevertheless, it is worth pointing out that the candidate SPEGs are made up of many single-pulse events with a peak-like shape found in their S/N versus DM curve, therefore, the probability of them being purely random should be significantly lower. . . . . . . . . . . . . . . .

4.24 Discovery of a pulsar J0539+0007 from the GBTDrift survey. The two SPEGs plotted in red in the bottom subplot are pulses emitted from this pulsar.

5.1 Different performance metrics for pulsar classification models trained and tested on GBTDrift. Notation: RandomForest (RF), AdaBoost (AB), SPEGnet $(\mathrm{SP}) \ldots \ldots \ldots \ldots \ldots$. . . . . . . . . . . . . 97

5.2 The PR and ROC curves of AdaBoost model trained on GBTDrift using 6-fold cross-validation. . . . . . . . . . . . . . . . . . . . 99

5.3 Recall, FPR, and $\mathrm{F}_{4}$ measure of three pulsar classification models within GBTDrift, for different sizes of training data. . . . . . . . . . . . . . 99

5.4 Performance metrics for pulsar classification models trained and tested on PALFA-extended. Notation: RandomForest (RF), AdaBoost (AB), SPEGnet $(\mathrm{SP}) \ldots \ldots \ldots \ldots$. . . . . . . . . . . . . . 101

5.5 PR and ROC curves of RandomForest model trained on PALFA-extended using 6-fold cross-validation. . . . . . . . . . . . . . . . . . . . 102

5.6 True Positive, False Negative, and False Positive values against different peak $S / N$ thresholds for RandomForest model trained and tested on PALFA-extended. . . . . . . . . . . . . . . . . . . . . . 102 
5.7 Recall, FPR, and $\mathrm{F}_{4}$ measure of pulsar classification models within PALFA-extended, for different sizes of training data. . . . . . . . . . . 103

6.1 Single-pulse search diagnostic plot of the discovery observation of pulsar J0327+09. Only one relatively bright pulse candidate (plotted in red) with peak $\mathrm{S} / \mathrm{N}$ of 7.79 is observed at $\mathrm{t} \sim 52.8 \mathrm{~s}$ in the beam. Compared with a standard single-pulse search diagnostic plot, the number of pulses versus $\mathrm{S} / \mathrm{N}$ subplot is omitted because it is not as informative as other subplots, especially for low $\mathrm{S} / \mathrm{N}$ pulsars. The comparison between the top and bottom two subplots shows the denosing effect of SPEGID. . . . . . . 109

6.2 Single-pulse search diagnostic plot of the discovery observation of pulsar J0342+27. Only one relatively bright pulse (plotted in red) with peak $\mathrm{S} / \mathrm{N}$ of 8.96 is observed at $\mathrm{t} \sim 52.0 \mathrm{~s}$ in the beam. . . . . . . . . 110

6.3 Single-pulse search diagnostic plot of the discovery observation of pulsar J1532+00. Only one relatively bright pulse candidate (plotted in red) with peak $\mathrm{S} / \mathrm{N}$ of 9.21 is observed at $\mathrm{t} \sim 30.3 \mathrm{~s}$ in the beam. . . . . . . 111

6.4 Single-pulse search diagnostic plot of the discovery observation of pulsar J2205+27. Four pulse candidates with underlying period of 2.0206 s were observed (plotted in red). The brightest candidate has peak $\mathrm{S} / \mathrm{N}$ of 7.03 is observed at $\mathrm{t} \sim 10.2 \mathrm{~s}$ in the beam. Note not all four candidates may be classified as pulsar by our model. However, because an underlying periodicity is found among these four candidates, their probability of being astrophysical is significantly increased. . . . . . . . . . . . . 112

6.5 Single-pulse search diagnostic plot of the discovery observation of pulsar $\mathrm{J} 1354+24$. Four pulse candidates with underlying period of $0.7794 \mathrm{~s}$ were observed (plotted in red). The brightest pulse candidate has peak $\mathrm{S} / \mathrm{N}$ of 7.93 is observed at $\mathrm{t} \sim 22.7 \mathrm{~s}$ in the beam. . . . . . . . . . . 113

6.6 Single-pulse search diagnostic plot of the discovery observation of pulsar $\mathrm{J} 2211+11$. Three pulse candidates with underlying period of $0.55756 \mathrm{~s}$ were observed (plotted in red). The brightest pulse candidate has peak $\mathrm{S} / \mathrm{N}$ of 7.1 is observed at $\mathrm{t} \sim 25.1 \mathrm{~s}$ in the beam. . . . . . . . . . 114

6.7 Single-pulse search diagnostic plot of the discovery observation of pulsar J0110+11. Eight pulses candidates (plotted in red) with underlying period of $0.43215 \mathrm{~s}$ were observed. The brightest pulse candidate has peak $\mathrm{S} / \mathrm{N}$ of 9.76 is observed at $\mathrm{t} \sim 0.05 \mathrm{~s}$ in the beam. It was missed by Clusterrank most likely because of the strong RFI contamination observed in the beam. The calculated period agrees with the periodicity found in the re-observation.115

6.8 Single-pulse search diagnostic plot of the observation of pulsar J2027+02. Four pulse candidates with underlying period of $1.6916 \mathrm{~s}$ were observed (plotted in red). The brightest pulse candidate has peak $\mathrm{S} / \mathrm{N}$ of 7.16 is observed at $\mathrm{t} \sim 11.3 \mathrm{~s}$ in the beam. This pulsar was later discovered by FAST telescope in August 2019. . . . . . . . . . . . . . . . 116

7.1 $\quad F_{4}$ measure between models trained only on the target GBTDrift data and those trained on the combined source and target training data (i.e., the whole PALFA benchmark data set and up to 20 pulsars from the GBTDrift data). Note that one pulsar corresponds to tens, even hundreds, of training SPEGs. . . . . . . . . . . . . . . . . . . 120 
7.2 Comparison of $F_{4}$ measure between models trained on the target PALFA data and those trained on the combined source and target training data (i.e., the whole GBTDrift benchmark data set and up to 20 pulsars from the PALFA data). Note that one pulsar corresponds to tens, even hundreds of training SPEGs. . . . . . . . . . . . . . . . . . . . . . 121

7.3 Comparison of transfer learning models (i.e., trAdaBoost and fine-tuning SPEGnet) with AdaBoost (trained on the combined data set without transfer learning) and SPEGnet models (trained on limited target data without transfer learning), when GBTDrift was used as target and PALFA-extended as source. Note that one pulsar corresponds to tens, even hundreds of training SPEGs. . . . . . . . . . . . . . . . . . 123

7.4 Comparison of transfer learning models trAdaBoost and fine-tuning SPEGnet with RandomForest (trained on the combined data set without transfer learning) and SPEGnet models (trained on limited target data without transfer learning), when PALFA-extended was used as target and GBTDrift as source. Note that one pulsar corresponds to tens, even hundreds of training SPEGs.

7.5 Histograms of the number of astrophysical SPEGs within in a beam from the GBTDrift and PALFA benchmark data sets. Note that the intervals of the histograms are left-closed (right open), i.e., the intervals of the histograms are: $[1,2),[2,4),[4,8)$, etc. . . . . . . . . . . 128

7.6 Histograms of the $\mathrm{S} / \mathrm{N}$ of the brightest astrophysical SPEG within a beam from the GBTDrift and PALFA benchmark data sets. Note that the intervals of the histograms are left-closed (right open) . . . . . . . . . . . . 129

8.1 Detection of a low S/N pulsar J1957+2831 in the PALFA survey on MJD 57024. The bottom DM versus time subplot only contains pulse candidates identified by our SPEGID algorithm [79]. The pulse candidates plotted in red are confirmed as they have similar DM and underlying periodicity as pulsar J1957+2831. . . . . . . . . . . . . . . . . . . . . 133

8.2 Proposed approach that calculates the weighted similarity between the target $\bar{T}$ and the candidate $\bar{X}$. Note that when we calculate the outlier score of a feature, only positively labeled SPEGs are considered. . . . . . 136

8.3 Number of low S/N pulsars detected versus number of recommendations. 143

8.4 Number of FRBs detected versus number of recommendations. . . . . . . 145 


\section{List of Tables}

2.1 The Weather Data Set with Some Numeric Attributes. Credit: [109] . . . 15

2.2 Confusion matrix for a classification problem. . . . . . . . . . . . . 24

4.1 Features extracted for each SPEG and used by machine learning algorithms for classification. . . . . . . . . . . . . . . . 68

4.2 List of machine learning algorithms used for the classification of SPEGs. . 69

4.3 The probability of finding an underlying periodicity among random singlepulse events. Tolerance level $(\alpha)$ reflects how consistent the time differences (between any two neighboring events) are with the predetermined period. Lower $\alpha$ value indicates higher consistency. Note that depending on the minimum period searched and the length of the data set (we used $268 \mathrm{~s}$ in our experiments), the values in this table may vary slightly. Nonetheless, the trend will be similar. . . . . . . . . . . . . 75

4.4 The results based on the benchmark data set by SPEG. . . . . . . . . . . 77

4.5 The performance metrics based on the benchmark data set by SPEG. . . 78

4.6 The results based on the benchmark data set by beam. . . . . . . . . . . . 79

4.7 The performance metrics based on the benchmark data set by beam. . . . 80

4.8 The results based on the full data set by SPEG. . . . . . . . . . . . . . 80

5.1 Additional Features extracted for each SPEG used by machine learning algorithms for classification. . . . . . . . . . . . . . . . 990

5.2 The number of beams with and without a pulsar and the number of SPEGs identified within them for the GBTDrift and PALFA-extended benchmark data sets. . . . . . . . . . . . . . . . . . . . . . 9 91

5.3 Classification results of different models trained on 100 and tested on 20 pulsars from the GBTDrift benchmark data set using 6 -fold cross validation. 96

5.4 Classification results of different models trained on 100 and tested on 20 pulsars from the PALFA-extended benchmark data set using 6-fold cross validation. . . . . . . . . . . . . . . . . . . . . 101

6.1 Comparison of the observing frequency and bandwidth between AO327 survey and GBT350 survey. . . . . . . . . . . . . . . . . . 108

7.1 Comparison of model performance between within-survey (trained and tested on GBTDrift, also shown in Table 5.3) and cross-survey (trained on PALFA-extended, tested on GBTDrift) predictions. . . . . . . . . . 118 
7.2 Comparison of model performance between within-survey (trained and tested on PALFA-extended, also shown in Table 5.4) and cross-survey (trained on GBTDrift, tested on PALFA-extended) predictions. . . . . . . 119

8.1 List of features extracted for each SPEG and used by the recommender system. . . . . . . . . . . . . . . . . . . . . 134

8.2 Ranking of features for detection of low $\mathrm{S} / \mathrm{N}$ pulsars. This rank is based on feature weights and is given in parentheses if that feature weight accounts for $<5 \%$ of the total weight. . . . . . . . . . . . . . . . 144

8.3 Ranking of features for detection of FRBs. This rank is based on feature weights and is given in parentheses if the feature weight accounts for $<5 \%$ of the total weight. . . . . . . . . . . . . . . 146 


\section{List of Acronyms}

PALFA

GBTDrift

PMPS

HTRU

AO327

ATNF

RRAT

FRB

RFI

DM

PRESTO

SPEG

SPEGID

$\mathrm{S} / \mathrm{N}$

DPG

NFL

RIPPER

PART

SVM

MLP

ANN

CNN

$2 \mathrm{D}$

DBSCAN

PCA

ILSVRC
Pulsar Arecibo L-band Feed Array

Green Bank Telescope Drift-scan

Parkes Multi-beam Pulsar Survey

High Time Resolution Universe

Arecibo all-sky $327 \mathrm{MHz}$

Australis Telescope National Facility

Rotating Radio Transients

Fast Radio Bursts

Radio Frequency Interference

dispersion measure

PulsaRExploration and Search Toolkit

Single-Pulse Event Group

Single-Pulse Event Group IDentification

signal-to-noise ratio

dispersed pulse group

no free lunch

Repeated Incremental Pruning to Produce Error Reduction

partial decision tree

Support vector machine

multilayer perceptron

Artificial Neural Network

Convolutional Neural Network

Two-Dimensional

Density-Based Spatial Clustering of Applications with Noise principle component analysis

ImageNet Large Scale Visual Recognition Challenge 
SMOTE

RUS

$\mathrm{TP}$

FP

TN

FN

FPR

FNR

$\mathrm{F}_{\beta}$

ROC

PR

DCG

IDCG

NDCG

TCA

TJM

DaNN

MMD

NLS

WLS

FLSC

DC

MI

RA

DEC

STEM

NSF
Synthetic Minority Oversampling TEchnique

random undersampling

True Positive

False Positive

True Negative

False Negative

false positive rate

false negative rate

$\mathrm{F}_{\beta}$ Measure

receiver operating characteristic

precision-recall

discounted cumulative gain

ideal discounted cumulative gain

normalized discounted cumulative gain

transfer component analysis

transfer joint matching

Domain Adaptive Neural Network

Maximum Mean Discrepancy

non-linear least squares

weighted least square

Fit Line SCore

dependence coefficienT

Mutual Information

right ascension

declination

science, technology, engineering, and mathematics

National Science Foundation 


\section{Chapter 1}

\section{Introduction}

As a subfield of astronomy, radio astronomy studies natural radio emissions that originate from celestial sources [16]. In 1932, Karl Guthe Jansky discovered natural radio emission from our Galaxy for the first time with a period of 23 hours and 56 minutes, which is the length of the sidereal day (the time it takes the Earth to rotate once relative to the background of the stars). After that, many discoveries have been made in the field of radio astronomy, including the discovery of pulsars in 1967 by Jocelyn Bell and Antony Hewish at Cambridge University [48]. Since then much progress has been made in the field of pulsar astronomy [59]. However, the advancement of modern technologies allowed development of larger and more sensitive instruments, consequently we face new challenges in finding pulsar signals from the large volumes of observed data. In this dissertation, we focus on developing machine learning approaches that aim at addressing these challenges, specifically on detection of radio pulsar signals in the time domain.

\subsection{Motivation}

Pulsars are rapidly rotating, highly magnetized neutron stars with extremely high density [59]. Neutron stars are the remnants of stars that are 8-20 times more massive than the Sun after stars end their lives in an explosion called a supernova. Neutron stars are made almost entirely of neutrons, and they rotate extremely rapidly after their formation due to the conservation of angular momentum. As pulsars rotate, charged 
particles are accelerated along the field lines of the magnetic field and a wide range of electromagnetic radiation from pulsars are observed. The study of radio pulsars provides useful information on a wide range of topics in astronomy and physics, such as neutron star physics, general relativity, the Galactic gravitational potential and magnetic field, the interstellar medium, and so on [59].

Pulsars are discovered through searching the data collected in pulsar surveys. An astronomical survey is a general map or image of a region of the sky during a certain period of time. Pulsar surveys can be either targeted or blind searches. The difference is that targeted searches only observe specific region of the sky, hence less data are collected. However, most pulsars have been discovered in blind searches. Since the discovery of the first pulsar, many pulsar surveys have been carried out aiming to discover more pulsars, such as the Parkes Multi-beam Pulsar Survey (PMPS) [66], the Pulsar Arecibo Lband Feed Array (PALFA) survey [18], the Green Bank Telescope Drift-scan (GBTDrift) pulsar survey [8], the Arecibo $327 \mathrm{MHz}$ (AO327) drift pulsar survey [25], etc. Once the radio data have been collected, the process of separating pulsars from human-generated radio frequency interference (RFI) and noise [59] is referred to as pulsar searching or pulsar candidate selection. It is estimated that there are over 100,000 detectable pulsars in the Milky Way [99]. However, currently there are just over 3,100 known pulsars in the Australia Telescope National Facility (ATNF) pulsar catalog [65].

The goal of pulsar searching is not only finding astrophysical signals in the data, but also identifying credible candidates that are worthy of further investigation (hence the name candidate selection). In early pulsar surveys, pulsars as well as promising candidates were found via the manual inspection of diagnostic plots conducted by domain experts. This was feasible because the number of diagnostic plots was generally very small. Today, as more and more data are collected in pulsar surveys, the number of diagnostic plots of pulsar candidates also increased significantly. Consequently, manual inspection of all the diagnostic plots become impractical. Different strategies have been used to automate the selection of credible candidates from candidate pools. Pulsar candidate selection is a challenging problem as it has strong prioritization of high recall, yet the pulsar search data is extremely imbalanced, i.e., pulsar signals only exist in a small fraction of all the observed data. This is because most pulsars are hundreds or even thousands of light years away, and therefore their signals are usually very dim when they are detected on Earth. Pulsar searching has often been compared to the problem of searching for needles in haystack [64, 28]. In addition to the class imbalance problem 
mentioned above, we face other challenges including but not limited to: a variety of signals with different characteristics caused by their inherent properties and current data processing techniques, building a fully labeled benchmark efficiently, the withinclass imbalance problem caused by rare cases such as low signal-to-noise ratio $(\mathrm{S} / \mathrm{N})$ pulsar signal, etc.

\subsection{Objectives}

The research in this dissertation aims to address the challenges of pulsar detection by proposing novel algorithms and approaches. Ideally, the developed automated singlepulse identification and classification approach should achieve close to $100 \%$ recall and relatively low false positive rate using machine learning.

The machine learning techniques used in this dissertation include unsupervised learning for pulsar identification, peak identification and scoring, feature engineering, supervised machine learning for classification, transfer learning for prediction across surveys, and recommender systems for detection of rare cases.

Specifically, the proposed algorithms and methods should be able to:

- overcome the challenges introduced by current data processing techniques,

- identify both bright and dim pulsar candidates from the diagnostic plots,

- separate pulse candidates from each other, and from RFI and noise as well,

- achieve as high recall as possible with low number of false positives at the stage of classification,

- produce classifiers with good performance by leveraging data from other pulsar surveys even when the training data are limited, and

- find rare cases in the data similar to an example provided by the user.

\subsection{Contributions}

The main algorithmic and methodological contributions of our research include the following:

- We developed a novel single-pulse candidate identification and classification approach, named Single-Pulse Event Group IDentification (SPEGID), which has very 
high recall $(>90 \%)$ and significantly reduces the number of pulse candidates requiring manual inspection (>90\%);

- A unique characteristic of the proposed pulsar identification approach is that it can identify not only bright, but also dim candidates and other types of challenging candidates (such as narrow pulses, pulses with multiple components, etc.);

- Our approach has significant denoising effect on the diagnostic plots, which aids easier and more efficient manual inspection;

- We developed a new peak-scoring algorithm that was able to detect the expected peak in the $\mathrm{S} / \mathrm{N}$ versus DM curve of an astrophysical pulse. This algorithm is able to account for a variety of pulse widths, shapes and brightness levels;

- The proposed algorithms and methods account for various challenges introduced by survey-specific observing parameters and current data-processing techniques. Therefore, our approach is universal and can be used on different pulsar surveys;

- We proposed a new algorithm to identify underlying periodicity among single-pulse search candidates detected at close DM;

- We investigated pulsar classification across surveys for the first time and showed that when there were limited training data, using transfer learning to extract useful information from the source survey improves single-pulse classification in the target survey; and

- We developed a novel hybrid recommender system to address the challenge of within-class imbalance and detect rare signals in the data, which constitutes a novel application of recommender systems.

We have applied the single-pulse search techniques developed during this research to re-analyze data collected in the GBTDrift survey and the PALFA survey and were able to discover eight new pulsars that had been missed by other pulsar searching approaches. Diagnostic plots in which these pulsars were discovered are shown in Chapters 4 and 6 . It can be seen from the diagnostic plots that these pulsars are difficult to find due to one or more of the following reasons: (1) there are only few pulses observed during the observation period; (2) the observed pulses have low $\mathrm{S} / \mathrm{N}$; and (3) there is strong RFI 
in the beam. This proves that our approaches are able to find these challenging pulsar signals that were often overlooked by other existing approaches.

Research work and results of this dissertation were published in two journals "Monthly Notices of the Royal Astronomical Society" [79] and "Publications of the Astronomical Society of the Pacific" [80], and at the highly selective data science conference "8th IEEE International Conference on Data Science and Advanced Analytics (DSAA 2021)" [81]. We implemented the algorithms in PYTHON and made the benchmark data and code available online in the Astrophysics Source Code Library (http://ascl.net/1807.014http://ascl.net/1807.014) and in a GitHub repository (https://github.com/dipangwvu/rare_case_detection).

\subsection{Organization}

The rest of this dissertation is organized as follows. We first describe the background on pulsar searches and machine learning in Chapter 2. We then present an overview of the related works in Chapter 3. Next, we present details of our two-stage single-pulse search approach SPEGID in Chapter 4. In Chapter 5, we discuss the improvement of classification stage of SPEGID using boosting and deep learning. We then present the results of re-analysis of AO327 survey in Chapter 6. We focus on pulsar classification across surveys using transfer learning in Chapter 7. We propose a novel hybrid recommender system as a solutions to the within-class imbalance problem using a hybrid recommender system in Chapter 8. Finally, we present our conclusions and the focus of future work in Chapter 9. 


\section{Chapter 2}

\section{Background}

In this chapter, we first give a brief description of pulsar astronomy. We then provide background on pulsar searches including data processing, periodicity and single-pulse searches, as well as data sets used in this dissertation. Also, we present background on machine learning techniques related to our research, including traditional machine learning (both supervised and unsupervised), transfer learning, imbalanced learning, recommender systems, and the performance metrics used for the evaluation of different learning techniques.

\subsection{Pulsar Astronomy Background}

Stars have a limited lifetime because the amount of hydrogen in their cores is limited. As the hydrogen within the cores of stars is depleted due to nuclear fusion, stars will then expand, cool and become red giants. After that, the path of the life cycle of a start is determined by its mass. While a small star like the Sun will turn into a white dwarf, a massive star will experience a supernova explosion. If the core of the star has a mass between 1.4 and 2-3 times the Sun's mass, the remnant of the explosion will be a neutron star. Stars with even larger masses will result in the formation of a black hole.

Pulsars are a type of neutron stars that have extremely high density, very strong magnetic fields and rapid rotations. As pulsars rotate, charged particles are accelerated along the field lines of the magnetic field and a wide range of electromagnetic radiation from pulsars is observed. If the magnetic poles of a neutron star do not coincide with its rotational axis, the rotation causes the beam to sweep across the sky (as shown in Figure 2.1). If the observer is in the path of the beam, pulses of radiation can be detected and hence the name "pulsar". Rotating radio transients (RRATs) are considered as a 


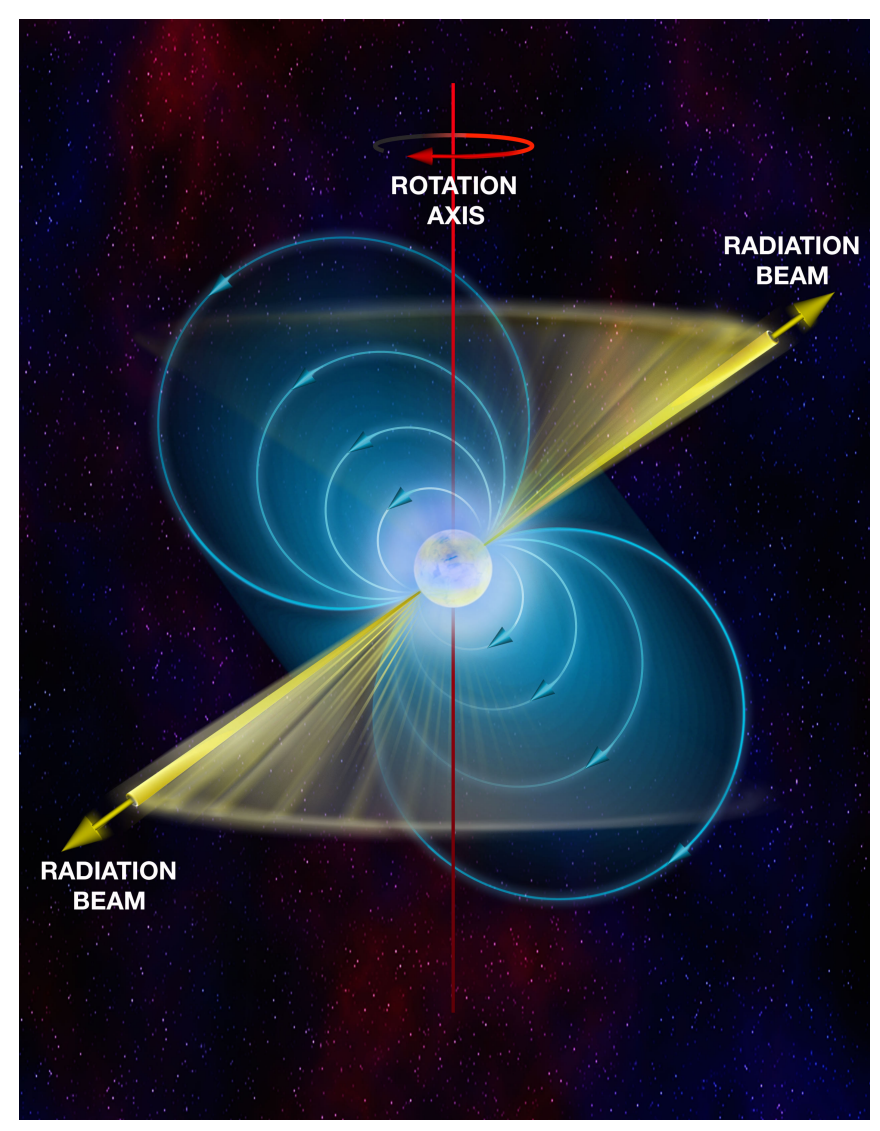

Figure 2.1: As a pulsar rotate, beams of radiation are emitted at its magnetic poles. Credit: B. Saxton, NRAO/AUI/NSF

special type of sporadically emitting pulsars [68]. Therefore the term "pulsar" is used to describe pulsars and RRATs throughout this dissertation.

\subsection{Pulsar Searches Background}

The discovery of pulsar starts from the collection of radio times series using radio telescopes as the one shown in Figure 2.2. Radio waves are a form a light, and they are reflected by the telescope dish and focused to the receiver, where they generate small electrical signals [64]. The raw voltage time series are then digitized and stored as raw telescope data. The raw data is then converted to filterbank files using a digital filter bank, which consists an array of band-pass filters. In this process, which is unsurprisingly called filterbanking, the digital signals are split into a number of frequency channels (shown in Figure 2.3). After that, pulsar signals, whether they are periodic or sporadic, are then searched using pulsar search and analysis software. One example of such soft- 
ware is PulsaR Exploration and Search Toolkit (PRESTO) developed by Scott Ransom [89].

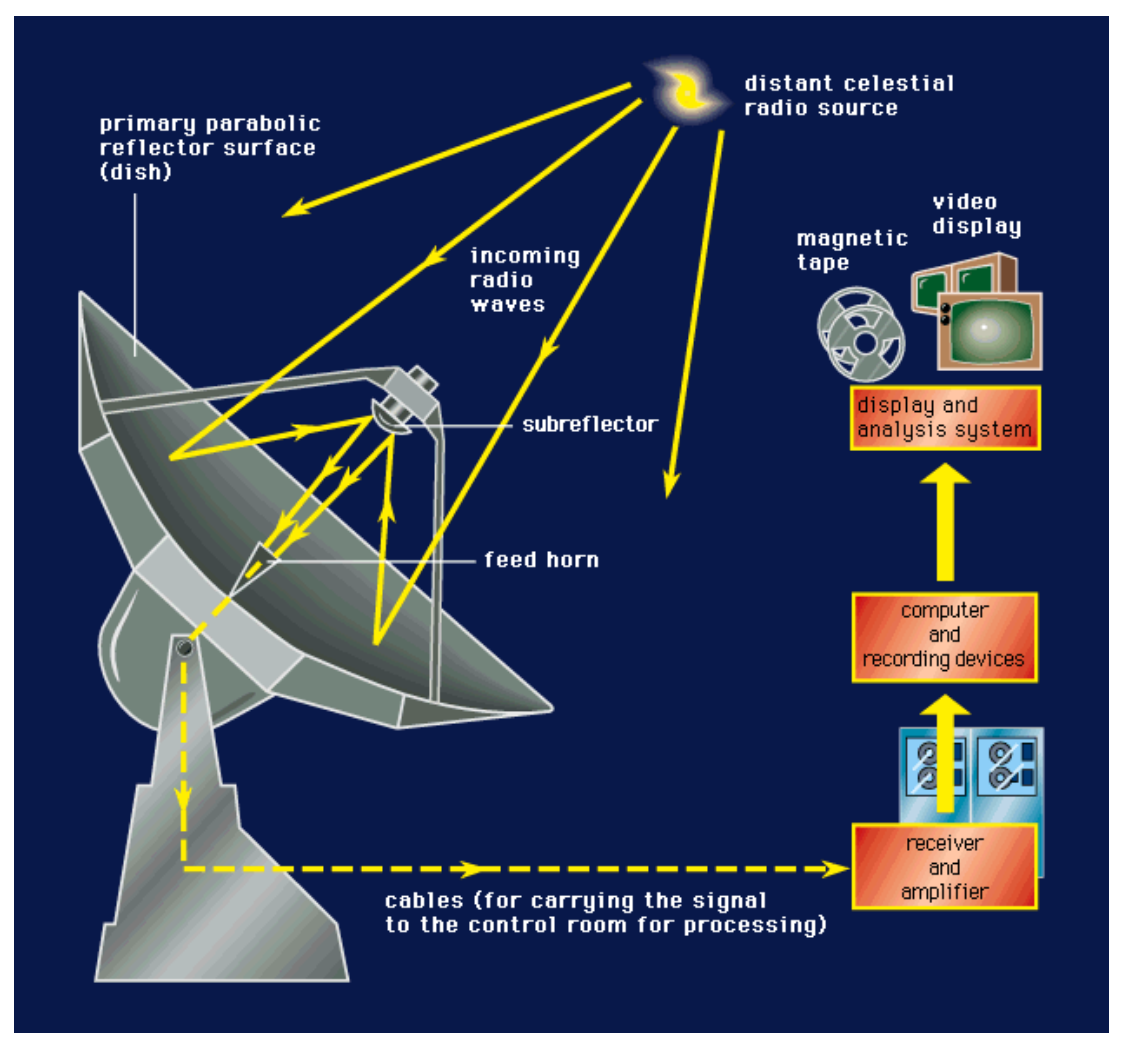

Figure 2.2: Diagram of a radio telescope. Credit: http://abyss.uoregon.edu/ js/ glossary/radio_telescope.html (accessed in November 2021).

\subsubsection{Pulsar Search Pipeline}

The major steps included in PRESTO, as shown in Figure 2.4, are RFI removal, dedispersion, periodicity and single-pulse searches, and inspection of candidates. Removing RFI signals is necessary because they are often much stronger than pulsar signals. Example of RFI removal techniques include clipping, which involves removal of extremely high power signals, and RFI masking, which ignores signals in certain frequency channels and/or certain time intervals. After that, the signals have to be dedispersed.

Dispersion refers to the phenomenon that, because of the interaction between signals and ISM, lower frequency components of signals arrive later than the higher frequency components. Hence the effect of dispersion has to be removed by dedispersion before the diagnostic plots are made [59]. Besides frequency, the magnitude of the delay also depends on dispersion measure (DM, which is defined as the integrated free-electron 


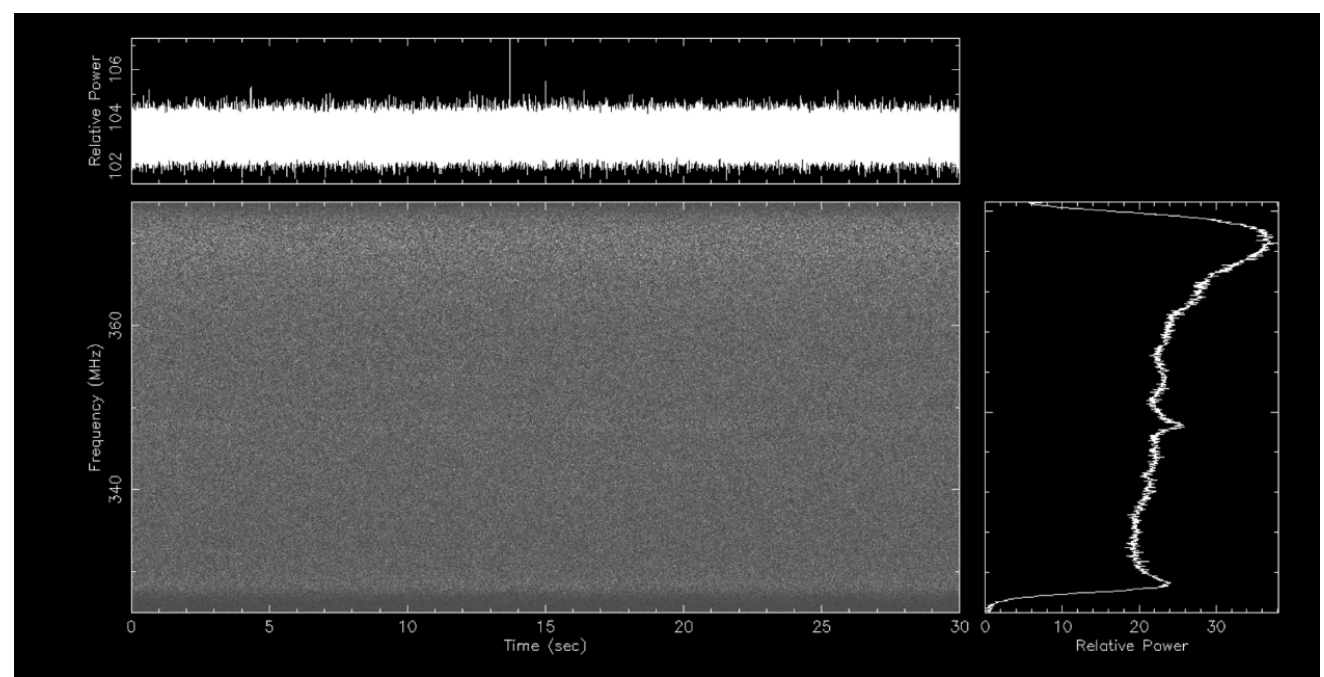

Figure 2.3: A graphical representation of a filterbank file. The top panel represents the time series and shows the observed power as a function of time. A filterbank is an array of bandpass filters that separates the input signal into multiple narrow frequency channels, which is shown as the frequency versus time subplot in the middle panel. The bandpass in shown in right panel and it demonstrates the observed power as a function of frequency. In other words, the time series shows the total power in all frequencies and the bandpass shows the power added over all times. Credit: [60]

column density along the line of sight in units of $\left.\mathrm{pc} \mathrm{cm}^{-3}\right)$. However, at the time of dedispersion, the true DM value of the source is unknown, therefore we must search for pulses across a range of trial DMs. After dedispersion, all frequency channels are added together, for a particular value of DM, to create a time series which is then searched for pulsar signals. Generally speaking, pulsar search approaches are divided into two categories: periodicity searches and single-pulse searches. While they both search the times series data for radio signals, periodicity searches look for periodic signals by first transforming the time series into the frequency domain using Fast Fourier Transforms (FFTs), and then folding the original time series at a period of interest to increase the $\mathrm{S} / \mathrm{N}$. In contrast, single-pulse searches look for bright, individual pulses without applying FFTs or folding [19]. Single-pulse searches are well suited for the discovery of isolated bursts that cannot be found in periodicity searches. Their application has led to the discoveries of RRATs [68] and Fast Radio Bursts (FRBs) [58], which consequently has renewed the interest in single-pulse searches [11]. These pulsar searching procedures are oftentimes implemented as pipeline software, such as PRESTO [89]. Both periodicity and single-pulse search pipelines can produce diagnostic plots for their identified candidates 
Outline of a PRESTO Search ${ }^{[84]}$

\footnotetext{
1) Examine data format (readfile)

2) Search for RFI (rfifind)

3) Make a topocentric, $D M=0$ time series (prepdata and exploredat)

4) FFT the time series (realfft)

5) Identify "birdies" to zap in searches (explorefft and accelsearch)

6) Make zaplist (makezaplist.py)

7) Make De-dispersion plan (DDplan.py)

8) De-disperse (prepsubband)

9) Search the data for periodic signals (accelsearch)

10) Search the data for single pulses (single_pulse_search.py)

11) Sift through the candidates (ACCEL_sift.py)

12) Fold the best candidates (prepfold)

13) Start timing the new pulsar (prepfold and get_TOAs.py)
}
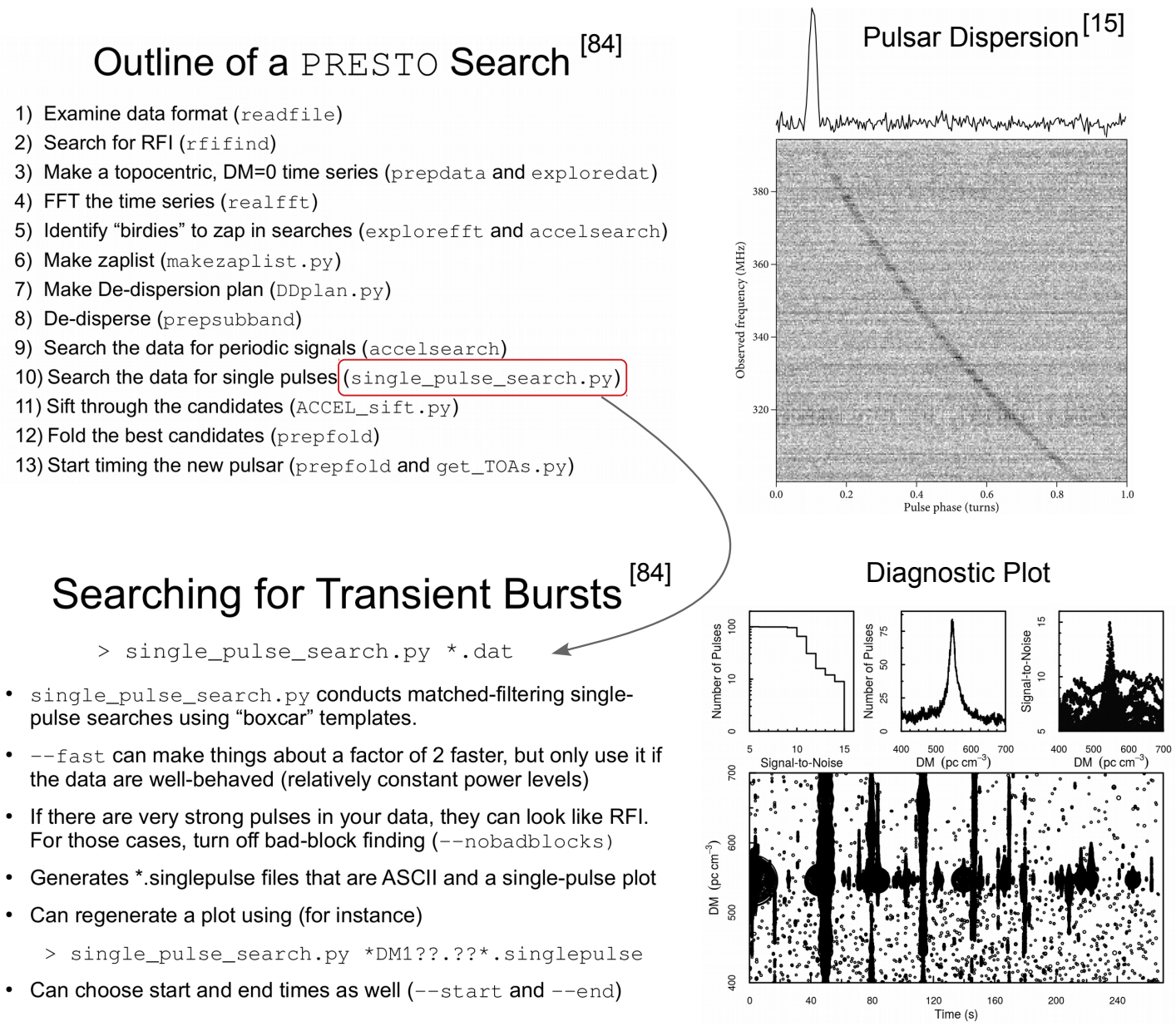

Figure 2.4: PRESTO-based single-pulse search pipeline. The outline of PRESTO search shows how the time series date are processed. The top subplot shows the dispersion of pulsar signals due to its interaction with ISM. The bottom panel shows in details how single-pulse search files are created and an example of single-pulse search diagnostic plot.

of interest. Two examples diagnostic plots for pulsars that are identified in periodicity search and single-pulse search respectively are shown in Figures 2.5 and 2.6. Typically a combination of information shown in these subplots is used to determine whether the candidate is astrophysical or not. In this work, we are particularly interested in finding pulsars through detection of their emission of individual radio pulses.

\subsubsection{Pulsar Search Collaboratory}

Since the discovery of the first pulsar in 1967 by Jocelyn Bell and Antony Hewish at Cambridge University [48], in early pulsar surveys, the diagnostic plots were manually 


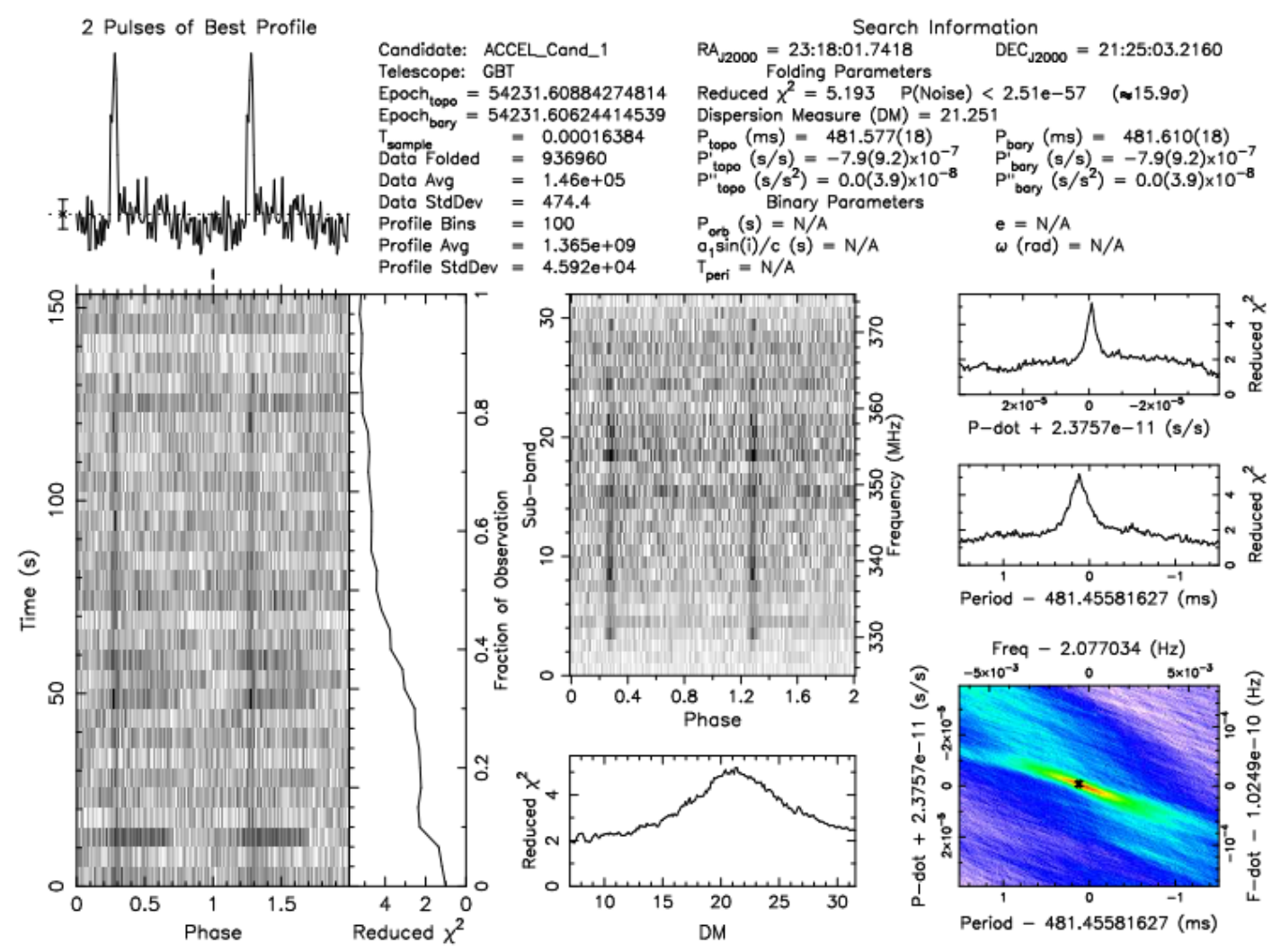

Figure 2.5: Diagnostic plot of signals from a periodic pulsar. This plots including subplots of the time series, pulse profile, the signal strength as a function of phase and observing frequency, the reduced $\chi^{2}$ versus DM, the reduced $\chi^{2}$ versus Period, the reduced $\chi^{2}$ versus P-dot, and the P-dot versus Period.

inspected because the number of diagnostic plots was relatively small. However, recent pulsar surveys collect more and more data because of improved telescope sensitivity, bandwidth and sky coverage and therefore more and more diagnostic plots are generated. For example, Lyon summarized the number of folded candidates in pulsar surveys and concluded there was an obvious upward trend that increased from thousands in 1970s to tens of millions in 2010s [64].

In the face of the large number of diagnostic plots, and to help promote the interest in the science, technology, engineering, and mathematics (STEM) among high school students in the United States, the National Radio Astronomy Observatory and West Virginia University started an NSF-funded program called Pulsar Search Collaboratory in 2008 to train high school students across the country to learn basic pulsar astronomy and help visually inspect the diagnostic plots [92]. Over the years, several new pulsars 

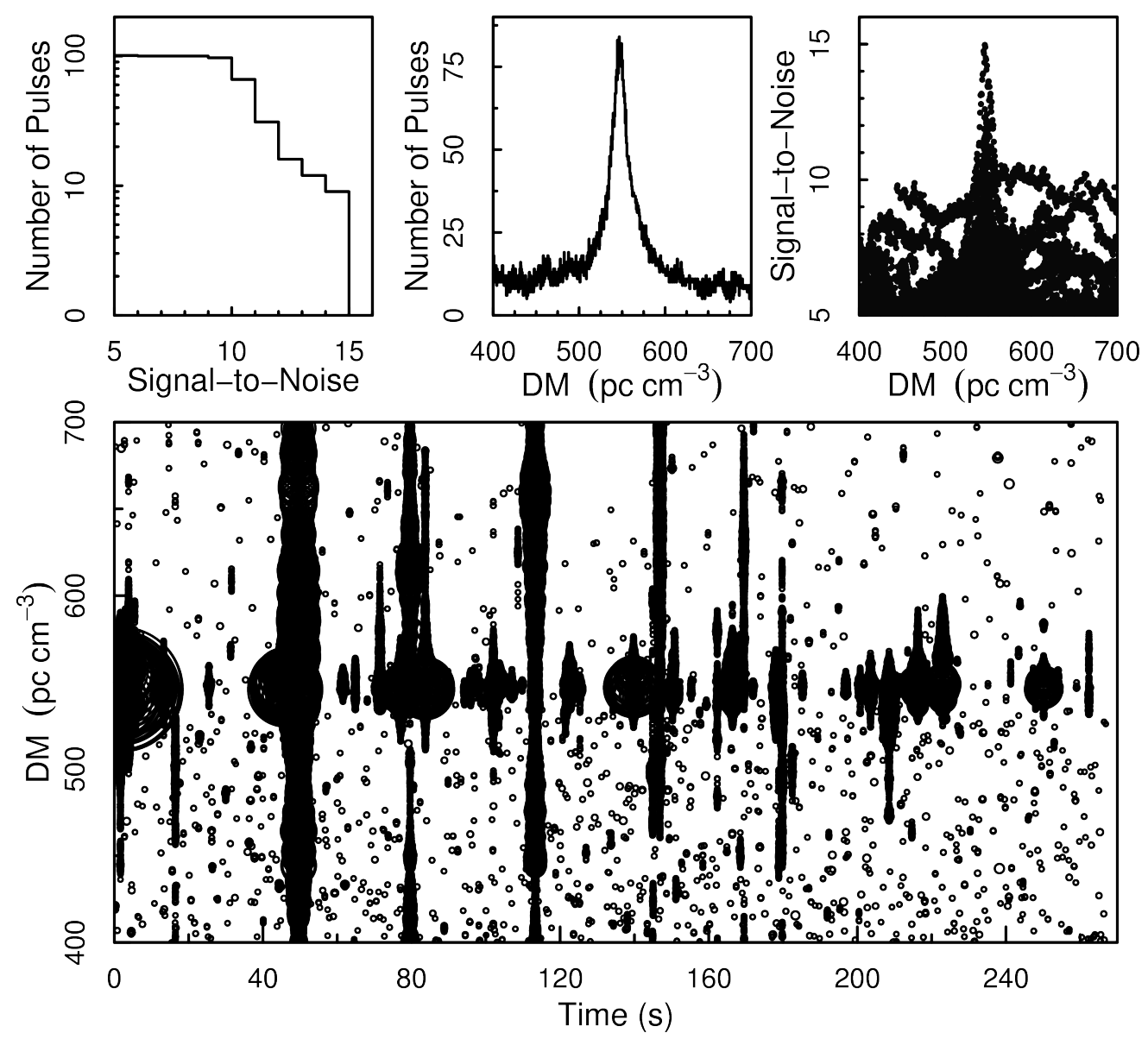

Figure 2.6: Known pulsar J1901+0355 detected on MJD 56245 in the PALFA survey. The four subplots include (clockwise from top left): a histogram of the number of singlepulse events versus $\mathrm{S} / \mathrm{N}$, a histogram of the number of single-pulse events versus DM, a scatter plot of $\mathrm{S} / \mathrm{N}$ versus $\mathrm{DM}$ and a scatter plot of the DM versus time for each single-pulse event whose size is proportional to its $\mathrm{S} / \mathrm{N}$.

have been discovered by the students before they graduate from high school [93]. This program also has demonstrated a positive impact on students' understanding of the nature of scientific inquiry and motivated many of them to pursue STEM career paths [7]. However, as recent pulsar surveys collect more and more data, it has become extremely time-consuming, if not impossible, to manually inspect all the diagnostic plots. Accordingly, many intelligent algorithms have been developed for the application of pulsar searching. 


\subsubsection{Radio Pulsar Data Sets}

In total three data sets are analyzed in the dissertation, which are derived from the PALFA survey, the GBTDrift survey, and the AO327 survey. Many more pulsar surveys can be found in the literature. The PALFA survey is an ongoing long-term pulsar survey started in 2004 [18]. This survey operates at a central frequency of 1.4 GHz. It uses the ALFA 7-beam receiver to search for pulsars close to the Galactic plane [99]. The GBTDrift survey was conducted with the Green Bank Telescope (GBT) from May through August in 2011 when it was immobilized for refurbishing. The receivers collected data at a radio frequency of $350 \mathrm{MHz}$ as the sky passed through the beam of the telescope [8]. The AO327 drift survey is a filler project as it collects data whenever the Arecibo telescope time becomes available, e.g., when scheduled observation cannot be conducted due to technical problems [25]. It collects data over the entire Arecibo sky (with the declination between -1 and 38 degree).

\subsection{Machine Learning Background}

\subsubsection{Definition of Machine Learning}

Nowadays, more and and more data and collected and recorded, not just in astronomy. However, information is hidden in the data, which can be potentially useful. Understanding the data is essential before we can make use of them. The process of automatically or semi-automatically discovering useful patterns in data that is stored electronically is called data mining [109]. The definition of learning and whether a computer can learn are philosophical questions, however, from the practical perspective, Mitchell defined that a computer program is said to learn if its performance at certain class of tasks improves with experience, and such experience is often referred to as training [72]. Similarly, Witten et. al gave an operational definition of learning: "Things learn when they change their behavior in a way that makes them perform better in the future." [109]. They further pointed out, this definition of learning emphasizes performance more than knowledge, which makes the learning more easily tested.

In practice, the purpose of a machine learning scheme is to generate a concept description (that can be understood, discussed, disputed, and applied to actual examples) from a set of instances [109]. As input of the machine learning scheme, each instance is characterized by the values of a series of predetermined features (also know as attributes). Therefore the data set that contains all the instances is represented by a 
matrix of instances versus features. Additionally, the input can also be unstructured data such as a group of images, text documents, or a time sequence. Learning in different applications can be divided into four categories: (i) classification learning when the goal is to classify the instances into different categories; (ii) association learning when the goal is to identify the association among features; (iii) clustering when the goal is to find groups formed by similar instances in the data; (iv) numeric prediction when the goal is to predict a numeric value. During the learning process, the concept is learned and the concept description is produced. Moreover, the concept description produced by different machine learning schemes can be different forms, e.g., tables, linear models, trees, rules, instance-based representation, clusters, etc. Alternatively, machine learning schemes can be divided as supervised learning, unsupervised learning, semi-supervised learning and reinforcement learning [74].

\subsubsection{Supervised Machine Learning Algorithms}

Both classification learning and numeric prediction are considered as supervised learning because each training instance comes with an actual outcome which provides the learning scheme supervision during the learning process. Given a set of labeled input-output pairs $\left\{\left(\mathbf{x}_{i}, y_{i}\right)\right\}_{i=1}^{N}$, the goals is to learn a function that maps inputs $\mathbf{x}$ to outputs $y$. Here $D$ is referred to as training set that contains $N$ training examples. If the response variable $y$ is categorical or nominal, the learning task is called classification. A classification example of deciding whether to play tennis based on the weather is shown in Table 2.1. On the other hand, when $y$ is real-valued, the learning task is numeric prediction, also known as regression.

Pulsar searching can be considered as a classification problem whose goal is to classify each pulsar diagnostic plots as containing pulsar signals or not. Nonetheless, unlike many typical classification problem, the goal of pulsar searching is to not only separate pulsar signals from non-pulsar signals (i.e., classification), but also to select credible candidates that are worthy of further investigation (e.g., re-observation which collects more data of the candidates by observing them for a longer period). Hence the problem is often referred to as pulsar candidate selection.

In the field of machine learning, the "no free lunch" (NFL) theorem states that two supervised machine learning algorithms (i.e., learners) on average exhibit equivalent performance across all possible problems $[110,40]$. In other words, no single machine learning algorithm is universally the best-performing algorithm for all problems. Due to 
the lack of research in single-pulse searches using machine learning at the time, it was necessary to test a wide range of machine learning algorithms on this problem. Therefore, in this dissertation, we mainly investigated the following machine learning algorithms: standard tree learner, direct rule learner, hybrid rule-and-tree learner, ensemble learner, support vector machine, artificial neural networks, and ensemble learning to classify single-pulse search candidates.

Table 2.1: The Weather Data Set with Some Numeric Attributes. Credit: [109]

\begin{tabular}{lllll}
\hline Outlook & Temperature & Humidity & Windy & Play \\
\hline Sunny & 85 & 85 & false & no \\
Sunny & 80 & 90 & true & no \\
Overcast & 83 & 86 & false & yes \\
Rainy & 70 & 96 & false & yes \\
Rainy & 68 & 80 & false & yes \\
Rainy & 65 & 70 & true & no \\
Overcast & 64 & 65 & true & yes \\
Sunny & 72 & 95 & false & no \\
Sunny & 69 & 70 & false & yes \\
Rainy & 75 & 80 & false & yes \\
Sunny & 75 & 70 & true & yes \\
Overcast & 72 & 90 & true & yes \\
Overcast & 81 & 75 & false & yes \\
Rainy & 71 & 91 & true & no \\
\hline
\end{tabular}

As data structure, a tree can have roots, branches and leaves. In data mining, trees can be used to summarize patterns found in data and establish classification or regression systems. For example, one decision tree can learned from the weather data set as shown in Figure. 2.7. J48 is a JAVA implementation of the C4.5 decision tree algorithm [87] and it constructs a decision tree using the divide-and-conquer technique. Specifically, attributes are tested sequentially and the instances are split up into subsets based upon the results of each test. These tests start from the attribute that results in subsets with the least total information, and continues with the attribute that results in subsets with the second least total information, and so on. If all instances at a node have the same classification (i.e., information is zero), the development of this part of the tree will stop. One advantage of the decision tree is that it can be easily interpreted by humans.

Rules is another common way of representing knowledge. A rule is comprised of two parts, the antecedent (i.e., a series of tests) and the consequent (the class), following a basic "if antecedent then consequent" structure. JRip, which is a JAVA implementation 


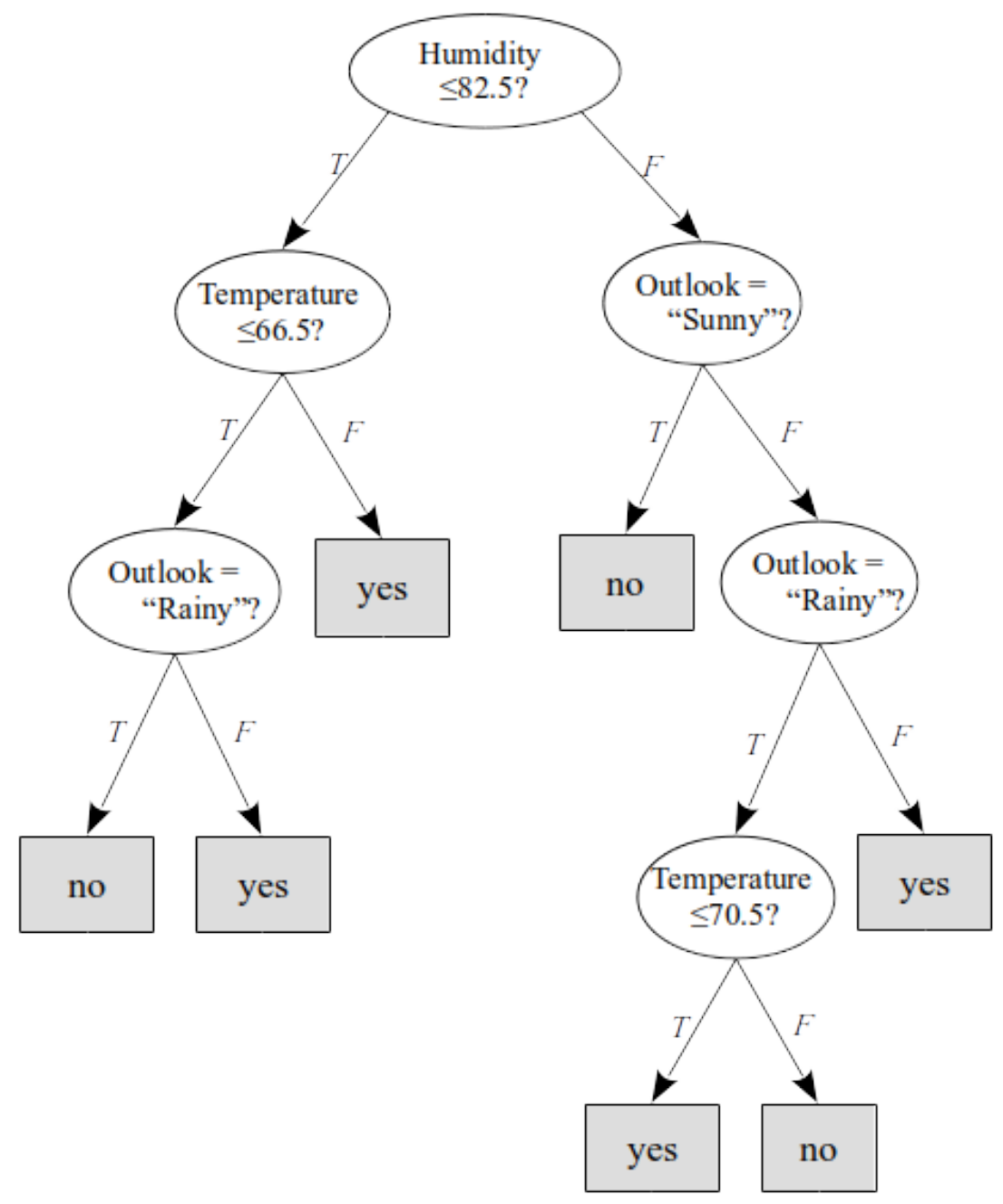

Figure 2.7: Decision tree for the weather data set.

of the RIPPER (Repeated Incremental Pruning to Produce Error Reduction) algorithm [15], is a direct rule learner that outputs learned knowledge as rules. JRip uses a separateand-conquer technique to identify rules covering instances from a specific class, separate them out, and continue on the remaining instances. To increase the overall accuracy of the learned rule set, the RIPPER algorithm uses incremental reduced-error pruning to generate rules and further revises them using a pruning data set to achieve global optimization. The learned rules can then be used to classify new instances. Like a decision tree, rules can also be easily interpreted.

PART is a hybrid rule-and-tree learner [36]. Like JRip, PART produces classification rules that are readily interpretable. However, unlike JRip, PART generates each rule by first building a partial decision tree, whose leaf with the largest coverage is made into a rule. After that, these covered instances are removed and more rules are created for the 
remaining instances, until all instances are covered. In other words, PART combines the divide-and-conquer technique for building a decision tree and the separate-and-conquer technique for producing rules. Obtaining rules from partial decision trees (instead of a fully explored one) avoids the problem of overpruning in a basic rule induction learner and also saves computation time.

Ensemble learning is also referred to as a committed method as it learns a weighted combination of multiple base models. Ensemble of weak learner is able to produce much stronger generalization ability than that of an individual learner. Nonetheless, the base learner does not have to be "weak learners" as in practice using not-so-weak learners often improves the performance of the ensemble [113]. Ensemble learning can be achieved in multiple ways. Among them the representative methods include bagging, boosting and stacking. Bagging refers to bootstrap aggregating. It first generates different training sets via bootstrapping and use them to fit different models, then combines the prediction of the models as the final outcome, which helps reduce variance and overfitting. RandomForest is a variant of the bagging method, which constructs a multitude of decision trees and outputs the average of their classification as the final prediction [9]. The key point is to reduce the correlation among the base learners (decision trees) by constructing each decision tree based on a randomly chosen subset of features extracted from a randomly chosen subset of instances. As a result, the variance of prediction would be greatly reduced and thus RandomForest models usually have good predictive accuracy. However, using multiple trees generally causes the loss of interpretability. Boosting is a machine learning approach built on the idea of creating a highly accurate prediction rule by combining many relatively weak and inaccurate rules. As an ensemble method, boosting improves the learning of a given algorithm by training weak learners sequentially, with each trying to correct its predecessor. AdaBoost is the first practical boosting algorithm developed by Freund and Schapire [37]. It boosts the accuracy of a weak learner through carefully adjusting the weights of training instances. If an instance is correctly classified, its weight will be decreased; otherwise its weight will be increased. Therefore, training instances that are hard to classify will become more important in the next iteration. In other words, boosting alters the distribution of the training data by changing the weights of the examples; hence it could be considered as a type of advanced sampling technique [105]. The final output is a weighted majority hypothesis in which the weight of each weak hypothesis is a function of its accuracy, and the overall generalization error will be significantly decreased. Different from bagging that produces an ensemble of homo- 
geneous classifiers, stacking focuses on building an ensemble of heterogeneous classifiers with a meta-learner. In other words, the outputs of the first-level classifiers are used as features for the second-level classifier [2].

Support vector machine (SVM) is a machine learning algorithms that can produce nonlinear class boundaries using linear models by transforming the data into a higher dimensional space using nonlinear mapping. After that, the algorithm tries to identify the maximum-margin hyperplane that separates different classes in the transformed space and simultaneously has the largest distance from the nearest data points (i.e., support vectors) on both sides. This maximum-margin hyperplane corresponds to a non-linear decision boundary in the original data space. Because SVMs are able to construct complex decision boundaries, they often produce classifiers with high accuracy. Generally speaking, SVM outputs weights of the features, and the weight of a specific feature indicates its importance in classification. However, decisions made by SVM are usually difficult to interpret from a human perspective. Furthermore, training SVM often takes a long time, especially when the data set is large. Another possible drawback is that overfitting may happen when the model becomes too complex. SMO is a type of SVM that implements the sequential minimal optimization algorithm for the training of the support vector classifier [86].

Deep learning adopts the hierarchy of concepts (i.e., a concept is defined in terms of its relation to simpler concept), therefore allows the learning of complicated concepts by building them out of simpler ones. Unlike most machine learning algorithms that are capable of discovering the mapping from features to output, deep learning solves the central problem in representation learning by introducing representations that are expressed in terms of other, simpler representations [41]. This way, deep learning is good at identifying the factors of variation that explain the observed data and hence alleviates the significance of feature design and is also robust to noise. The representative example of a deep learning model is multilayer perceptron (MLP). MLP is a class of feed forward Artificial Neural Network (ANN) [74]. ANNs identify non-linear decision boundaries of data by including many perceptrons (nodes or artificial neurons) that are organized into multiple layers in the network. Each perceptron uses a non-linear activation function. Such layering allows the building of complex concept and makes MLP well suited for solving complex learning problems. However, MLP models are usually difficult to interpret and they usually require a large amount of training data.

Over the years, the performance of deep learning models have improved consistently. 


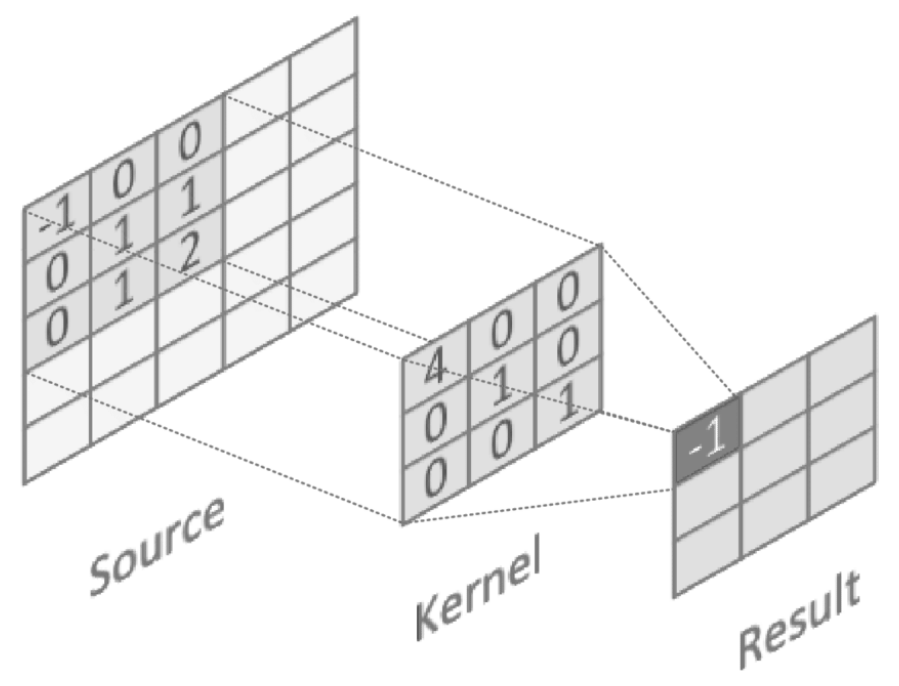

Figure 2.8: Schematic of 2D convolution.

Yet it was until 2012, when a convolutional neural network (CNN) won the ImageNet Large Scale Visual Recognition Challenge (ILSVRC) by a wide margin, the interest in deep learning increased significantly [54]. One advantage of convolution is it leverages sparse interactions, parameter sharing and equivariant representations. More specifically, the convolution operation of two functions is denoted as

$$
s(t)=\int x(a) w(t-a) d a,
$$

or

$$
s(t)=(x * w)(t),
$$

where $\mathrm{x}$ is often referred to as the input and $\mathrm{w}$ is referred as the kernel. Just like ANN loosely models the neurons in a biological brain, the architecture of CNN emulates a part of the brain called primary visual cortex. Neurophysiologists observed that neurons in the early visual system of cats actively responded to very specific patterns of light, but hardly to other patterns [41]. Similarly, the front layers of CNN often detect small meaningful features such as edges which the back layers use to build more complicated concepts [112]. Note that convolution with one single kernel can only extract one type features, therefore in CNN the convolution often involves multiple applications of convolution in parallel. An example of $2 \mathrm{D}$ convolution is shown in Figure. 2.8. In recently years, CNNs have achieved many successes in object recognition, detection, and segmentation. Examples are shown in Figure. 2.9. Note that compared to object recognition (also known as image classification), object detection is more difficult as it requires localization of objects first, 


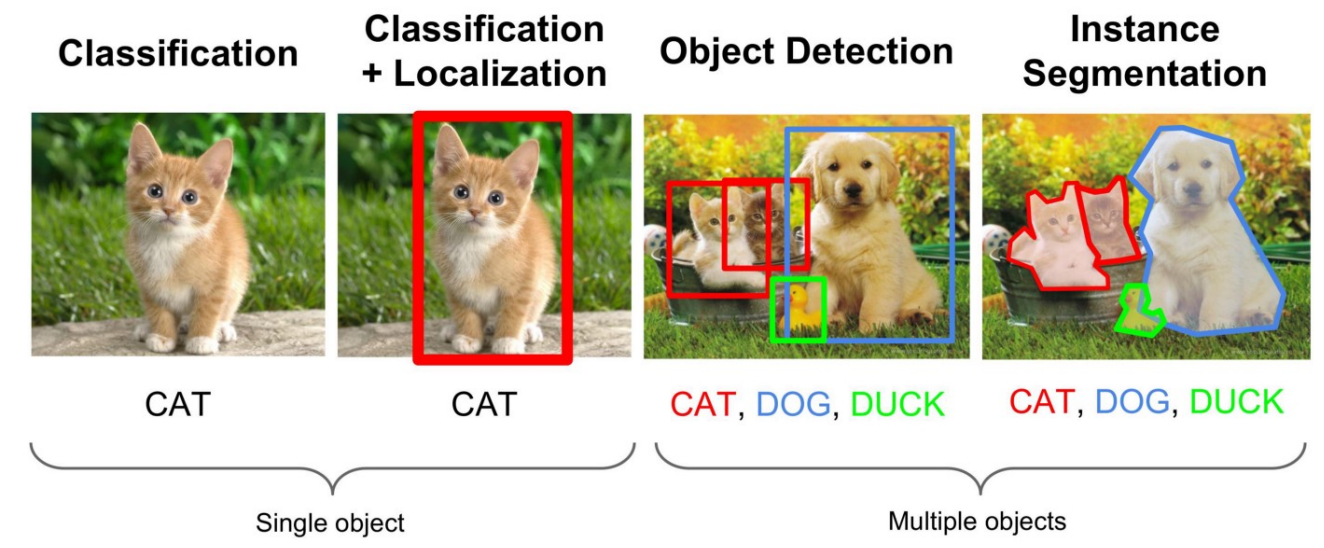

Figure 2.9: Comparision between image classification, objection detection and instance segmentation. Credit: [76].

which is often realized by combining CNNs with region proposals. More details about object detection models using CNN can be found in [98, 46].

\subsubsection{Unsupervised Machine Learning Algorithms}

Unsupervised learning is also known as descriptive learning. In this problem, data points are not associated with labels, and the goal of learning is to find structures of the data such as clusters or latent variables. A latent variable is a variable that is not directly measured but rather inferred from other variables that are directly measured.

One representative unsupervised learning is clustering, which involves grouping data objects with similar properties together [45]. Clustering is very useful in finding previously unknown groups in a data set. The similarity (and therefore, dissimilarity) between data objects is usually determined by the distance measure derived from their attributes. Clustering algorithms are divided into two categories: partitioning (non-hierarchical) and hierarchical. While partitioning methods separate data objects into a number of clusters, hierarchical methods link the cluster pairs successively and thus form a nested hierarchy among clusters [90]. Examples of partitioning methods include K-means clustering and Density-Based Spatial Clustering of Applications with Noise (DBSCAN), whereas examples of hierarchical methods include agglomerative and divisive clustering.

Another application of unsupervised learning is discovering latent variables. This is often used to reduce the dimensionality of high dimensional data hence is often referred to as dimensionality reduction. The idea is to project the data into a lower dimensional space of latent factors that capture most of the variability of the data. The applica- 
tion includes data visualization and noise reduction. Common dimensionality reduction techniques include principle component analysis (PCA), auto-encoder, etc.

\subsubsection{Transfer Learning}

An interesting observation of machine learning is that a dumb algorithm with a lot of training data oftentimes outperforms a clever algorithm with modest amounts of data [29]. However, in practice collecting training data is often expensive or difficult. Therefore, using data from that can be more easily collected from different domains can help produce better models. On the other hand, traditional machine learning assumes that training data, on which the model are built, have the same input feature space and the same (marginal and conditional) distribution as the testing data. When this assumption is not met, the results of the trained model can be degraded. As a result, transfer learning has been studied to improve the transferring of information from a related domain to the target domain [78, 108].

Transfer learning can be categorized in different ways. For example, based on whether the target task is the same as the source task, transfer learning can be divided into: (i) inductive transfer learning (the target task is different from the source task); (ii) transductive transfer learning (the target task is the same as the source task); (iii) unsupervised transfer learning (the target task is different from but related to the source task) [78]. Based on the form of information transfer, transfer learning can be divided into: (i) instance-based; (ii) feature-based; (iii) parameter-based; (iv) relational-based [108]. In recent years, as deep learning achieved significant improvements in many applications, deep transfer learning has also been studied to reduce the amount of training data and training time in the target domain [100].

\subsubsection{Imbalanced Learning}

Machine learning problems often involve learning from imbalanced data, which is often problematic for many standard learning algorithms as they generally assume that all the classes in the training data are equally represented [21]. As a result, classifiers tend to perform much better on the majority class than on the the majority class $[105,106]$. However, in reality rare classes (and rare cases) usually provide more information than common classes (and common cases) hence are often more interesting.

Specifically, when the number of instances belonging to the majority class significantly exceeds that of instances belonging to the minority (i.e., rare) class, the data set 
is considered to be imbalanced [106]. This type of imbalance is defined as class imbalance, or more specifically between-class imbalance. On the other hand, if a small subset of instances form a sub-concept within a class, they are called rare cases and this type of imbalance is called within-class imbalance.

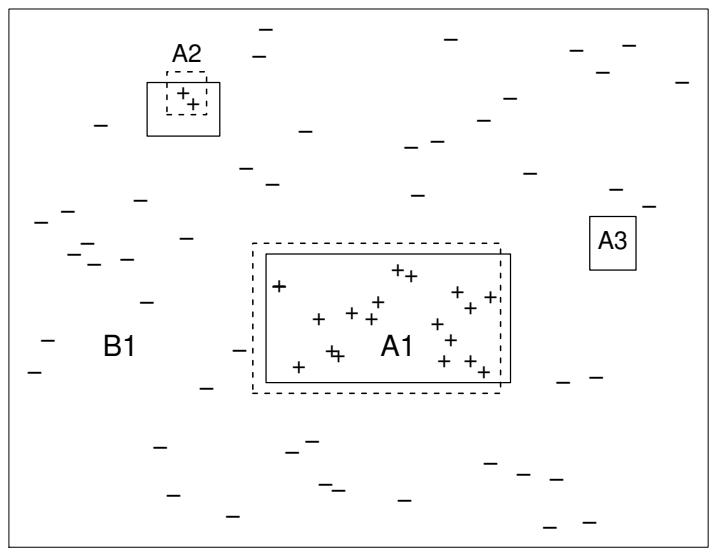

Figure 2.10: Graphical representation of rare class and rare cases.

An illustration of these two types of imbalance problems is shown Figure. 2.10 [105]. In this figure, A represents the minority (positive) class, and B represents the majority (negative) class. The true decision boundaries are shown as the solid lines while the learned boundaries are shown as the dashed lines. More specifically, A1 corresponds to the relatively common instances, A2 corresponds rare cases learned from the training examples, and A3 represents the missed rare cases due to the lack of similar examples in the training data. Thus, the positive class contains subconcepts that cover small groups of rare cases (i.e., A2 and A3) that are different from the majority of positive training examples. A group of the rare cases form a separate disjunct, which usually has a higher error rate caused by noise and small training set size. Due to the lack of training examples, both rare classes and rare cases make supervised learning more difficult. Accordingly, the development of algorithms that are robust to different types of imbalance has been studied and properly termed as "imbalanced learning" [106]. It should be noted that within-class imbalance caused by rare cases is not as extensively studied as between-class imbalance. Additionally, the problem gets even more difficult when the within-class imbalance is coupled with class overlapping and noise [21].

Different types of imbalanced learning techniques have been developed, such as: (i) using sampling methods to modify the class distribution of the data. Representative work includes random oversampling, random undersampling, Synthetic Minority 
Oversampling TEchnique (SMOTE), ect.; (ii) assigning different costs for misclassifying particular data examples, i.e., cost-sensitive methods. Representative work includes applying misclassification costs to the data set as a form of dataspace weighting, incorporating cost-sensitive functions or features directly into classification paradigms, etc.; (iii) using active learning, which is a family of machine learning methods that query the data instances to be labeled for training by an expert or oracle; (iv) using one-class learning, which is designed to learn a concept using mainly a single class of examples rather than learning to distinguish instances of the positive and negative classes. More details of the techniques can be found in [47].

\subsubsection{Recommender Systems}

Recommender systems refer to software tools and techniques that can provide users suggestions for items to be used [91]. The development of recommender system technology has been driven by the increasing importance of the Web as a medium for electronic and business transactions. By providing users with personalized suggestions, recommender systems are helpful in alleviating the problem of "information overload". Today recommender systems have been successfully used to help user find items that may be of interest to them, from movies, electronics, houses to academic papers [2]. In practice, the recommendation problem can be formulated either as a prediction problem in which the goal is to predict the rating value for a user-item combination, or as a ranking problem, in which the goal is to recommend the top k-items for a particular user. Based on the type of data that they deal with, recommender systems are mainly categorized as collaborative filtering, content-based recommender systems, knowledge-based recommender systems, and hybrid systems [2].

Collaborative filtering methods make recommendations to the active user based on the user-item interactions of other users with similar tastes [82]. In contrast, contentbased methods use the attribute information about the items and recommend items that are similar to those the user liked in the past [69]. Knowledge-based methods, similar to content-based systems, also make recommendations based item attributes. However, a significant amount of knowledge is incorporated in finding the similarity between items. Based on the user interactive mode and the corresponding knowledge bases used to facilitate the interaction, knowledge-based recommender systems can further be categorized into: (i) constraint-based recommender systems, in which users specify their requirements or constraints on the item attributes, and a set of rules is used to match the 
user requirements with item attributes. (ii) case-based recommender systems, in which specific cases are given by users to represent their interests or preferences, and similarity metrics defined on the item attributes are used to discover matching items [10]. One important characteristic of cased-based recommender systems is that the returned recommendations can be pruned using directional critiques, which are often specified as constraints of attributes of special interest [2].

Different types of recommender systems use different sources of data, and have different advantages and disadvantages. For example, collaborative filtering systems require a significant amount of data to make accurate recommendations, hence they perform poorly for new users and new items, which is referred to as the cold-start problem [2]. By contrast, knowledge-based systems suffer less from this issue as they are not dependant on historical data. In order to combine strengths of different methods and avoid their weakness, hybrid recommender systems have been designed. One example is a meta-level hybrid system called "collaboration via content", in which a content-based system was first used to learn each user's profile as a vector of discriminative features and the recommendation was then made in a collaborative way [97].

\subsubsection{Performance Metrics}

\subsubsection{Performance Metrics for the Evaluation of Classifications}

To measure and compare the effectiveness of the classification, we calculated several performance metrics that are derived from the confusion matrix shown in Table. 2.2 [109].

Table 2.2: Confusion matrix for a classification problem.

\begin{tabular}{lll}
\hline & Predicted positive & Predicted negative \\
\hline Actual positive & True positive $(\mathrm{TP})$ & False negative $(\mathrm{FN})$ \\
Actual negative & False positive $(\mathrm{FP})$ & True negative $(\mathrm{TN})$ \\
\hline
\end{tabular}

The meanings of the four terms in the confusion matrix are as follows: (i) True Positive (TP): the number of SPEGs that are correctly classified as pulsars; (ii) False Positive (FP): the number of SPEGs that are incorrectly classified as pulsars; (iii) True Negative (TN): the number of SPEGs that are correctly classified as non-pulsars; (iv) False Negative (FN): the number of SPEGs that are incorrectly classified as non-pulsars.

A good learner produces classifiers with a high number of true positives, i.e., a low number of false negatives. Moreover, a low false positive number is also preferred as it 
reduces the effort required for manual inspection. For each classifier, we calculated the following performance metrics.

(i) Recall: the number of positive training instances correctly classified over the total number of positive training instances. Recall is also referred to as the true positive rate (TPR).

$$
\text { Recall }=\frac{\mathrm{TP}}{\mathrm{TP}+\mathrm{FN}}
$$

(ii) False negative rate (FNR): the percentage of positive training instances misclassified as belonging to the negative class.

$$
\mathrm{FNR}=\frac{\mathrm{FN}}{\mathrm{FN}+\mathrm{TP}}
$$

(iii) Precision: the number of positive training instances classified correctly over the total number of instances classified as members of the positive class.

$$
\text { Precision }=\frac{\mathrm{TP}}{\mathrm{TP}+\mathrm{FP}}
$$

(iv) False positive rate (FPR): the percentage of negative training instances misclassified as belonging to the positive class.

$$
\mathrm{FPR}=\frac{\mathrm{FP}}{\mathrm{FP}+\mathrm{TN}}
$$

The performance metrics mentioned above are commonly adopted in learning with balanced class distribution. However, when working with imbalanced learning, the following three performance metrics are strongly recommended: (i) receiver operating characteristic (ROC) curve, (ii) precision-recall (PR) curve, and (iii) $\mathrm{F}_{\beta}$ - Measure [96].

ROC curve summarizes the performance of classifiers over a range of true positive rates and false positive rates and therefore can be used measure the performance of a classifier at different thresholds. On the other hand, when the goal is to improve recall without hurting precision, the $\mathrm{PR}$ curve can provide a more informative picture of a learner's performance than the ROC curve [22]. The area under ROC and PR curves are commonly used to compare the performance of different classifiers. $\mathrm{F}_{\beta}$ - Measure is family of metrics that characterize the trade-off between TP, FP, and FN. It outputs a single value that represents the how well a classifier performs in the presence of rare classes. In Equation 2.7, the value of $\beta$ reflects the relative importance of precision and recall. Higher $\beta$ value indicates indicate stronger preference for high recall over high precision. When $\beta$ is not specified, $\mathrm{F}_{1}$ score is assumed.

$$
\mathrm{F}_{\beta}=\frac{\left(1+\beta^{2}\right) \times \text { Precision } \times \text { Recall }}{\beta^{2} \times \text { Precision }+ \text { Recall }}
$$




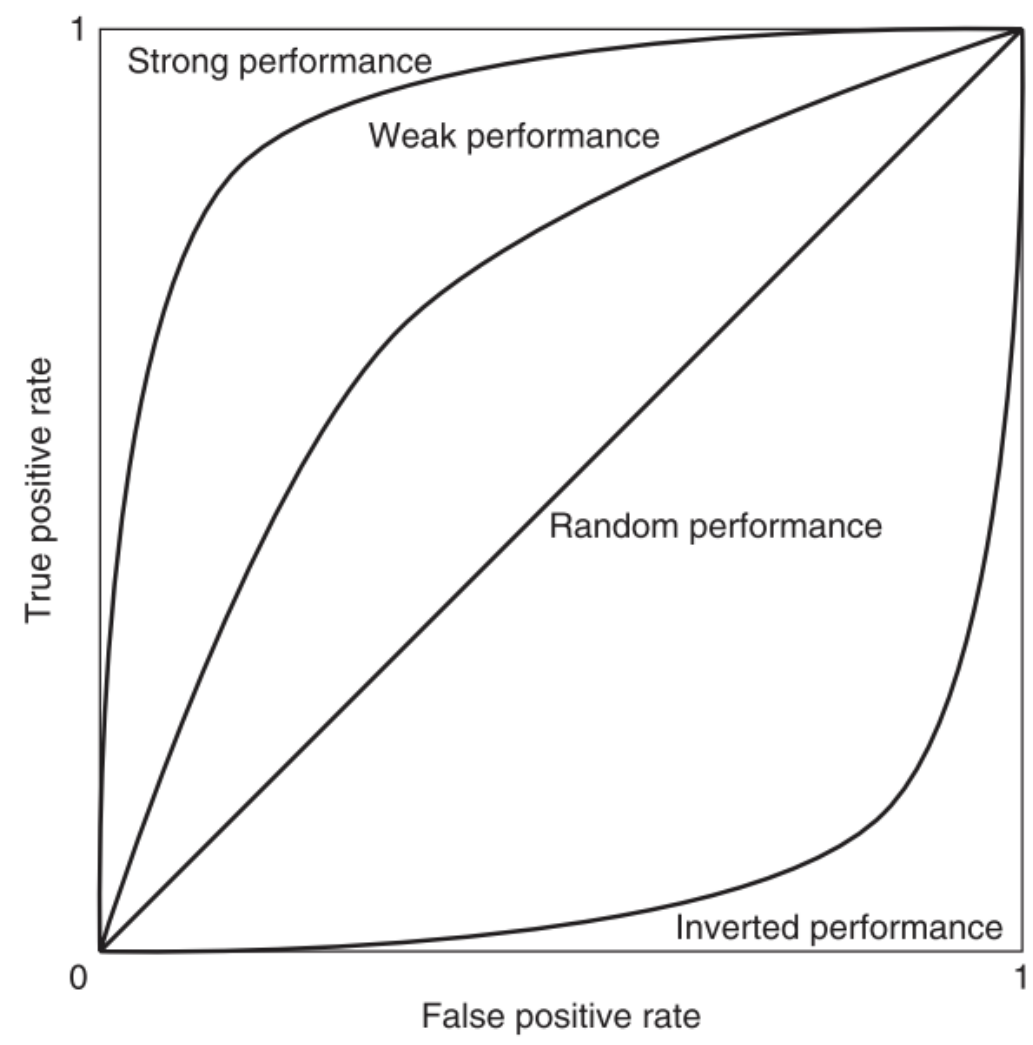

Figure 2.11: Examples of ROC curves. Each curve represents the performance of a different classifier on the given data set. Credit: [96]

\subsubsection{Performance Metrics for Evaluation of Recommender Systems}

For for evaluation of recommender systems, accuracy is still one of the most fundamental measures. Accuracy can be used to evaluate either the predicted ratings or the top-k ranking. Furthermore, other secondary measures such as coverage, novelty, diversity, etc. also provide complementary information. When the goal is to detect rare cases, because the use only insects the the top-k items rather than all the items, the true usefulness of the recommender system is better reflected by ranking-based measures evaluations. The ordering of test items predicted by the recommender system is compared with the ground-truth ranking via correlation or utility [2]. While the most commonly used rank correlation coefficients are Spearman rank correlation coefficient, Kendall rank correlation coefficient, etc., the utility-based methods measure the utility of a particular ranking by the relevance of recommended items, which should be high in the recommended list. More specifically, the ground-truth rating is used in combination with the recommender system's ranking to define the utility function of an item $j$ to the 
user $u$ :

$$
F(u, j)=\frac{\max \left\{r_{u j}-C_{u}, 0\right\}}{2^{\left(v_{j}-1\right) / \alpha}} .
$$

Here the numerator is the rating-based utility, in which $r_{u j}$ is the (hidden) ground-truth value of the rating and $C_{u}$ is a break-even (neutral) rating value for user $u$, and the denominator is the ranking-based utility of the item, in which $v_{j}$ is the rank of the item in the recommendation list, and $\alpha$ is a half-life parameter. Equation (2.8) shows that increased rank of an item results in exponentially decay of its utility, which indicates strong preference of the top few items. The sum of the utility of all items in the test set (namely $I_{u}$ ), is defined as the R-score:

$$
\operatorname{R} \text {-score }(u)=\sum_{j \in I_{u}} F(u, j) \text {. }
$$

Although the rank of an item $v_{j}$ can be any value from 1 to $\mathrm{n}$ (where $\mathrm{n}$ is the total number of items), in practice, the size of the recommended list is often limited to a predetermined values $L$. Therefore, items ranking below $L$ are considered having zero utility to the user. Consequently, the R-score of the recommended list of size $L$ is:

$$
\mathrm{R} \text {-score }(u)=\sum_{j \in I_{u}, v_{j} \leq L} F(u, j) .
$$

In a scenario where items ranking below $L$ may also be of interest to the user, exponential day in the utility can be too restrict. Therefore, a milder discount rate, namely the discounted cumulative gain (DCG), has been proposed as the following:

$$
D C G=\sum_{j \in I_{u}} \frac{g_{u j}}{\log _{2}\left(v_{j}+1\right)} .
$$

where $g_{h j}$ is the utility (or gain) of the item $j$ to the user $u$ which is typically set to an exponential function of the relevance of the item for the user as follows:

$$
g_{u j}=2^{r e l_{u j}}-1
$$

For the case of implicit feedback data sets, based on whether an item is consumed by the user, the relevance is assigned as 1 or 0 . Similarly, when the discounted cumulative gain is computed over a recommendation list of size $L$ :

$$
D C G=\sum_{j \in I_{u}, v_{j} \leq L} \frac{g_{u j}}{\log _{2}\left(v_{j}+1\right)} .
$$

To compare DCG over different data sets, the normalized discounted cumulative gain (NDCG) is defined as ratio of DCG and the ideal discounted cumulative gain (IDCG, 
i.e., the discounted cumulative gain of the ground-truth rankings):

$$
N D C G=\frac{D C G}{I D C G} .
$$

One advantage of NDCG over rank-correlation coefficients or ROC is its ability to distinguishing higher-ranked items from lower-ranked opponents [2]. 


\section{Chapter 3}

\section{Related Work}

This chapter presents a survey of the literature related to our work. The first section presents pulsar classification methods used in periodicity searches, the second section reviews single-pulse search approaches, the third section reviews related work on transfer learning, and the last section describes related work on data mining with rarity.

\subsection{Related Work on Periodicity Searches}

As mentioned in Chapter 1, detection of pulsars in periodicity searches is essentially an image classification problem as each diagnostic plot only relates to an individual pulsar candidate. Consequently, automated pulsar searching approaches, which ranging from scoring algorithms to convolutional neural networks (CNN), were first developed in periodicity searches as it is more straight forward compared with single-pulse searches.

Due to the large number of candidates, Faulkner et al. [33] developed a graphical candidate selection tool called REAPER to aid the classification of candidates. However, this approach simply removed all candidates with $\mathrm{S} / \mathrm{N}<8$, and all other candidates were manually examined. Later, Keith et al. [53] proposed a candidate scoring method based on a series of heuristics that test for pulsar-like signal of the signal. Using this candidate selection technique, they were able to discover 28 pulsars that were initially missed in the Parkes Multibeam Pulsar Survey (PMPS) data.

Eatough et al. [30] presented the first method to automatically identify credible candidates from pulsar surveys using an artificial neural network (ANN) with one hidden layer. The ANN was trained on a data set that includes 259 known pulsar signals and 1625 non-pulsar signals. The trained ANN was able to identify about $92 \%$ of pulsars in a test sample of $\sim 2.5$ million pulsar candidates. Later, Bates et al. [5] and Morello 
et al. [73] also proposed their pulsar candidate classification methods using ANN with different feature designs respectively.

The advancement of field image classification also resulted in improvements of pulsar classification periodicity searches, one technique that is especially worth noting is CNN. Unlike the above-mentioned ANNs, CNN is able to automatically extract meaningful features from the diagnostic plots and therefore eliminates the necessity of complicate feature design.

Zhu et al. [114] presented a two-level artificial intelligence (AI) program that classify pulsar candidates based on features extracted from the time versus phase plot and frequency versus phase plot and hand-crafted features extracted from other 1D subplots. Their trained model was able to rank all the pulsars within the top $1 \%$ of all candidates.

Recently, Guo et al. [43] enhanced the performance of CNN-based pulsar identification by comining a deep convolution generative adversarial network (DCGAN) with a support vector machine (SVM). In their framework, the DCGAN was used as a sample generation (to overcome class imbalance problem) and feature learning model, whereas the SVM was used for the classification of candidates. Their results showed the accuracy was improved.

\subsection{Related Work on Single-pulse Searches}

While machine learning approaches have been extensively applied in periodicity searches, machine learning techniques concerning single-pulse detection were lacking until recently [26]. This is because detection of pulsars in single-pulse search diagnostic plots, like other object detection problems, consists of two stages, i.e., pulsar candidates in the diagnostic plots need to be localized first before they can be correctly classified. Furthermore, the brightness of astrophysical pulses, as well as RFI may vary significantly in different observed beams. Therefore, single-pulse searches impose a challenge, especially for detection of dim pulsars in the presence of RFI.

Cordes and McLaughlin [19] first described the theoretical background for detecting isolated radio pulses, which considered issues pertaining single-pulse searches such as the effects of propagation through intervening ionized media including dispersion, scattering, and scintillation. They suggested a single-pulse search pipeline consisting of dedispersion, match filtering, thresholding and diagnostics. Since then the pulsar candidates were manually inspected, although many efforts had been invested to reduce the RFI in the diagnostic plots $[23,52]$. 
Like periodicity searches, ranking was first adopted in single-pulse searches before any automated method was developed. Compared with manually inspecting all candidates one by one, a more efficient approach is to first rank the candidates and then based on their astrophysical features only examine the best candidates manually. A representative approach of this type is RRATtrap, proposed by Karako et al. [50]. In their algorithm, single-pulse events at similar DMs and times were first placed into groups. Each group was then assigned a rank using a set of predetermined rules related to its astrophysical features. Finally, only diagnostic plots that included groups with ranks higher than a threshold were inspected. This approach was able to reduce the number of diagnostic plots that required manual examination by an order of magnitude. However, because Radio Frequency Interference (RFI) and true pulsar signals can be similar in appearance and the shapes of pulsar signals can vary significantly, it is difficult to find a set of efficient, predetermined rules that universally work.

Devine et al. [26] presented the first machine learning approach to single-pulse searches. This approach first identified pulsar signal candidates by finding dispersed pulse groups (DPGs) via inspecting how their $\mathrm{S} / \mathrm{N}$ varied with $\mathrm{DM}$ using a peak-identification algorithm called Recursive Algorithm for Peak IDentification (RAPID). It then classified DPGs automatically using supervised machine learning. This approach greatly reduced the number of candidates that needed to be examined manually. However, the implementation only made use of the composite $\mathrm{S} / \mathrm{N}$ versus DM subplot for an entire observation, meaning that dimmer pulses could be hidden by brighter pulses or RFI and hence overlooked.

Recently, Michilli et al. [70] developed a machine learning classifier, namely Singlepulse Searcher (SPS), to discriminate astrophysical signals from RFI with high-speed and accuracy. Their approach grouped single-pulse events that are close in the DM-time space to for a pulse candidate, and classify the candidates using Gaussian-Hellinger Very Fast Decision Tree. However, like RRATtrap, the closeness of single-pulse events are also based on predetermined time and DM intervals. We will show in this proposal that this type of grouping approaches are inherently not robust to classify diagnostic plots generated by current data processing pipeline. Furthermore,

In order to successfully apply machine learning in single-pulse searches, several additional challenges have to be addressed. The first challenge is related to ubiquitous RFI signals, which vary drastically in properties and brightness. Furthermore, some RFI signals look like astrophysical pulses, making it difficult to distinguish them. Second, the 
automated approach should be able to detect a variety of pulses. This is because astrophysical pulses can vary significantly in brightness, width, and shape, sometimes even for pulses from the same source. Third, data processing techniques lead to additional challenges. For example, variable spacing of trial DMs [19] and clipping [89] can change the appearance of pulses in the $\mathrm{S} / \mathrm{N}$ versus DM space, as well as in the DM versus time space, making their identification more complicated. Lastly, the large number of candidates calls for an efficient machine learning approach with low false positive rate and even lower false negative rate. In summary, there is still much to improve in automated single-pulse search approaches.

Note that deep learning has been applied to searches in the frequency-time domain [17], but these searches are limited to moderately bright pulses which can be detected across the frequency band. Time-domain searches and hence our approaches are aimed at finding not only bright, but also dim pulses (which would not be detectable in frequency versus time domain plots), hence making our work complementary to the work of [17]. On the other hand, note that in order to detect narrow pulses, the sampling time of the time series is often set at the level of tens of microseconds. Therefore, the resolution of the DM versus time subplot is so high that conventional object detection approaches used in computer vision are not appropriate for this problem.

It is worth noting that single-pulse searches in the frequency-time domain, as have recently been used to discover FRBs, use similar image classification techniques to pulsar periodicity searches [104]. Their main similarities and differences are as follows.

- In both periodicity searches and frequency-time single-pulse searches candidates are generated automatically. Each candidate is independent from other candidates and therefore the detection of astrophysical signals is equivalent to the classification of the diagnostic plots. As a result, candidate selection in periodicity searches and frequency-time searches can be considered as a standard image classification problem. Accordingly, many image classification techniques (including Convolutional Neural Networks) can be applied [114, 17].

- The spectral information of astrophysical signals is very helpful for discriminating candidates from frequency-time searches from RFI or thermal noise. However, only bright signals (with $\mathrm{S} / \mathrm{N}$ greater than roughly 8 ) are detectable across the multiple frequency channels necessary for this discrimination [17]. Our work is particularly aimed at finding both bright and dim pulses, making our approaches complementary. 
- A large number of candidates (in the order of millions) are generated in periodicity searches [73, 63] and frequency-time FRB searches [17]. On the other hand, in time-domain single-pulse search approaches (including SPEGID), the diagnostic plot of each beam can be considered as a candidate plot (from which single-pulse candidates can be identified). Therefore, the number of candidates in time-domain single-pulse search approaches is much smaller (on the order of tens or hundreds of thousands).

\subsection{Related Work on Transfer Learning}

Traditional machine learning assumes that training data and test data having the same input feature space and the same data distribution. When there is significant difference between the training and test data, the performance of models often degrades. In other cases, obtaining training data may be difficult and/or expensive. Therefore, transfer learning, which can help solve the problems of degraded performance and limited training data simultaneously, has been successfully applied to many fields [78, 108]. Transfer learning refers to the process of improving the learning of target predictive functions in the target domain by transferring information from a related source domain. Transfer learning can be categorized as homogeneous transfer learning, where the input feature space of the source domain and the target domain is the same (i.e., $X_{S}=X_{T}$ ), or heterogeneous transfer learning in cases where $X_{S} \neq X_{T}$. Furthermore, homogeneous transfer learning can be divided into four categories: instance-based, feature-based, parameter-based, and relational-based [108].

Dai et al. [20] developed an instance-based transfer learning framework named trAdaBoost which is based the boosting learning algorithm [37]. trAdaBoost uses a small amount of labeled in the target domain to leverage labeled data in the source domain to build models with high performance. Later, La et al. [55] improved the weighting strategy of the boosting algorithms and their approach could use the training data more effectively.

Pan et al. [77] proposed a feature-based transfer learning method called transfer component analysis (TCA), for domain adaptation. TCA learns transfer components that do not cause significant distribution change across the domains. In other words, data distributions in different domains are close to each other in the subspace spanned by these transfer components. Long et al. [56] developed the transfer joint matching (TJM) approach to achieve feature matching and instance reweighting in a unified opti- 
mization problem. Like TCA, TJM reduced the domain difference by constructing new features representation that is invariant to the distribution difference across domains. Nonetheless, it is worth noting domain adaptation explicitly assumes that the data from the source and target domains are of different marginal probabilities $\left(P\left(X_{S}\right) \neq P\left(X_{T}\right)\right)$, but similar conditional probabilities $\left(P\left(Y_{S} \mid X_{S}\right) \approx P\left(Y_{T} \mid X_{T}\right)\right)$ [42] [44].

Recently, deep learning has received increasing attention as it is able to learn highlevel features automatically. However, one drawback of deep learning is that it often needs a lot more training data than traditional machine learning methods to avoid overfitting [41]. Therefore, deep transfer learning has been developed accordingly. Yosinski et al. [111] presented the first study on tranferability of features from each layer of a neural network. They found that the generalization performance of a deep neural network can be improved with transferring features and fine-tuning. Ghifary et al. [39] proposed a Domain Adaptive Neural Network (DaNN) which incorporated the Maximum Mean Discrepancy (MMD) measure as a regularization of the cost function to reduce the distribution mismatch between the source and target domains in the latent space. The idea of adding adaptation layers and an additional domain confusion loss to to learn a domain invariant representation was later adopted in many neural network architectures $[101,57]$.

Transfer learning has been successfully applied in several areas of astronomy. Gupta et al. [44] combined domain adaptation with active learning to classify supernova Ia. Their approach used neural networks as the learning algorithm, and Kernel Mean Matching as the domain adaptation technique to leverage information from the source data set. They found that the best strategy was to combine domain adaptation with poolbased active learning with margin sampling. Ackermann et al. [1] used transfer learning to detect galaxy mergers. They first trained the deep CNN on the IMAGENET data set and then train the network with the galaxy merger data. Their result showed that transfer learning outperformed random initialization, and their classifier significantly outperformed previous methods for automated visual detection of galaxy mergers. However, to the best of our knowledge, transfer learning has not yet been used for single-pulse searches in the time domain.

\subsection{Related Work on Mining with Rarity}

Class imbalance is a common problem in supervised machine learning. Without any imbalance treatment, machine learning models tend to err in favor of the majority 
class and classify all instances as "non-pulsars". While such models exhibit very high accuracy, they have no practical use due to the high false negative rate. It is known that data collected in pulsar surveys are extremely imbalanced as only a small fraction of the observed beams contain pulsar signals, which form the very class that we are interested in. To overcome the class imbalance problem, different resampling methods, including sampling, data fragmentation, etc. have been developed [31].

The class imbalance has not received much attention in the field of pulsar searching until recently. In periodicity searches, [73] used oversampling of the pulsar class to overcome the class imbalance of the training set. Guo et al. [43] used the deep convolution generative adversarial network to generate samples generation to overcome class imbalance problem. In single-pulse searches, Devine et al. [26] tested three different imbalance treatments including oversmapling, SMOTE [14] and undersampling on their benchmark data. Their results showed that SMOTE outperformed other resampling approaches for imbalance treatment. SMOTE often outperforms oversampling by introducing new, nonreplicated minority class examples that results in additional generalization.

However, we will show in this proposal that in pulsar searches, there is not only class imbalance, but also within-class imbalance [105]. While the former has been well recognized in previous studies, the latter has been less acknowledged and results in greater challenges. Within-class imbalance are caused by rare cases within the positive class, which are examples that are infrequently observed and define a small region of the instance space. The subconcept represented by rare cases are often more difficult to learn due to the small training set size.

Rare cases, as they are different from the majority of the instances in the same class, tend to form sub-clusters during the learning process, where classification errors tend to be concentrated most heavily [21, 105]. The problem of small disjuncts was firstly found in rule-based learning, and corresponds to rules that only cover a small group of data points, but now it is known to be caused by sub-clusters within classes [21].

To over this problem, different approaches have been proposed. Weiss [105] summarized techniques that had been used to improve the mining with rare cases, such as adjusting of the bias of a learner, employing boosting, placing rare cases into separate classes, etc. Das et al. [21] presented an updated review of research on data irregularities (distribution-based as well as feature-based) which not only included class imbalance, small disjuncts, but class skew, missing features, and absent (non-existing or undefined) features as well. In addition, they pointed out that different data irregularities were 
often observed to be violated together by data sets, hence their interrelation and cooccurrences of the data irregularities were also discussed. Notably, they mentioned that overlapping could result in poor learning in the vicinity of the region of overlap. This problem has received relatively little attention in the literature. More details about on mining with rare cases can be found in these two survey papers and references therein.

In practice, in all categories of pulsar searches (including periodicity searches and frequency-time and time-domain single-pulse searches), astrophysical signals are found only in a small fraction of candidates. For time-domain single-pulse searches, our SPEGID only produced one diagnostic plots from which single pulse signals can be identified. Generally speaking, a pulsar (including both known and new discoveries) with one or more pulses could be found in every 100 to 500 diagnostic plots (i.e., beams). For periodicity searches, each candidate is obtained by folding a dedispersed time series at a specific period. Therefore, for each beam, tens or even hundreds of candidates are generated by folding at different periods of interest [63]. Because pulsar signals are only observed in a small percentage of beams, and periodic signals can only be detected at certain specific periods, the imbalance ratio between pulsar class and non-pulsar class in periodicity searches can be extremely small. For example, the periodicity search candidates produced in a subset of the PMPS data had a class imbalance of roughly 1:11,000 [62]. The imbalance ratio for FRB detections in single-pulse search data is even lower as FRB events are even rarer [17]. Consequently, it is hard to form a consensus on an optimal imbalance ratio when creating a benchmark data set. Different imbalance ratios have been used to create different benchmark data sets for different categories of pulsar searches (e.g., 1:75 for the High Time Resolution Universe (HTRU) periodicity search benchmark data [73], and 1:1 for the FRB deep learning benchmark data sets [17]). To alleviate the adversarial effect of the imbalance on the model performance, most studies included the imbalance treatment if the benchmark data were highly skewed $[73,26,6,79]$. Likewise, in this study, the training data (not the test data) were treated with SMOTE or random undersampling (RUS) to obtain 1:1 ratio of astrophysical SPEGs to non-astrophysical SPEGs. Additionally, the effect of the imbalance ratio on models' testing performance can be mitigated by selecting adequate performance metrics. 


\section{Chapter 4}

\section{SPEGID: Identification and Classification of Pulsar Signals}

This chapter presents a novel, two-stage approach, named Single-Pulse Event Group IDentification (SPEGID), which combines unsupervised and supervised machine learning to automatically identify and classify single pulses in radio pulsar search data ${ }^{1}$. In the first stage, we identify astrophysical pulse candidates in the data as trial single-pulse event groups (SPEGs) by clustering single-pulse events and merging clusters that fall within the expected DM and time span of astrophysical pulses. We also present a new peak scoring algorithm, to identify astrophysical peaks in $\mathrm{S} / \mathrm{N}$ versus DM curves. Furthermore, we group SPEGs detected at a consistent DM as they are likely emitted by the same source. In the second stage, we first create a fully labeled benchmark data set derived from the PALFA survey by selecting a subset of data with SPEGs identified (using stage 1 procedures), extract their features and manually labeled individual SPEGs. We then use the benchmark to train classifiers via supervised machine learning. Next, using the best trained classifier, we automatically classify unlabeled SPEGs identified in the full data set. To aid the examination of dim SPEGs, we develop an algorithm that searches for an underlying periodicity among grouped SPEGs.

\subsection{Approach Outline}

Figure 4.1 shows the outline of our proposed two-stage approach in a flowchart, with numbers in parentheses indicating the corresponding section in which the details are described. Briefly speaking, the first stage of our approach, namely SPEGID, includes

\footnotetext{
${ }^{1}$ The work presented in this chapter was published in [79].
} 


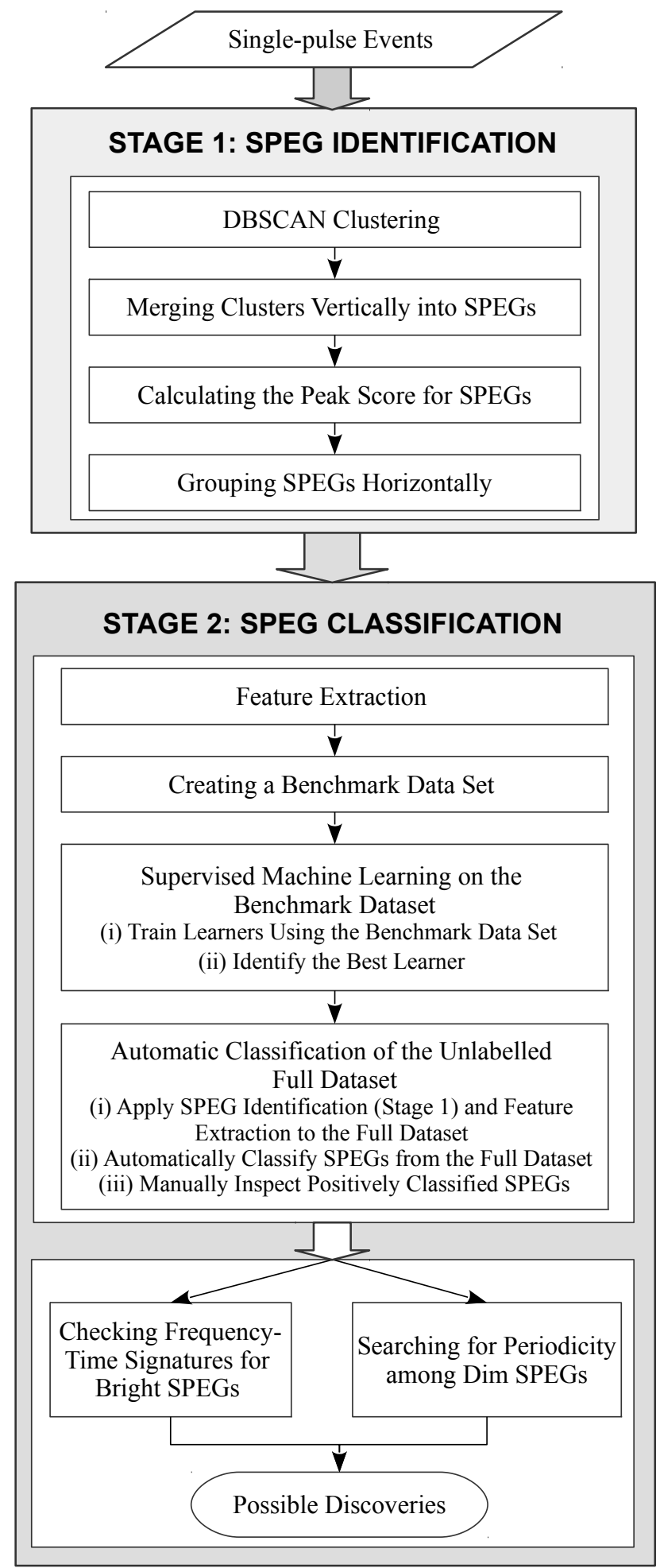

Figure 4.1: The outline of our proposed approach SPEGID. 
the identification of pulse candidates as SPEGs that are made up of related single-pulse events, the characterization of the $\mathrm{S} / \mathrm{N}$ versus DM curve of SPEGs and the recognition of the association among them. One benefit of stage 1 is that it removes most noise events and some RFI and, therefore, significantly denoises the DM versus time space of the diagnostic plots. Consequently, the manual inspection of the denoised diagnostic subplots (with SPEGs identified) would require much less effort compared to the original DM versus time subplots. However, when the number of diagnostic plots is large, manual inspection would still be tedious and time-consuming. Therefore, instead of manual classification, we use the output from the first stage as input to the second stage to automatically classify the identified SPEGs as pulsar and non-pulsar events.

The novel aspects of SPEGID include the following:

1. We successfully identified pulse candidates as SPEGs in the DM versus time subplots by first applying Density Based Spatial Clustering of Applications with Noise (DBSCAN) (i.e., unsupervised learning) [32] on single-pulse events and then merging the clusters based on the relations among their S/N, width, DM offset (i.e., the absolute difference between the trial DM and the true DM) [19] and time drift. Note that clustering and merging of clusters are two integral parts for the identification of pulse candidates as SPEGs.

2. We developed a new peak scoring algorithm which is capable of identifying the peak in the $\mathrm{S} / \mathrm{N}$ versus DM curve of pulses/SPEGs with different brightness, width, and shapes. This algorithm also accounts for variable spacing of trial DMs.

3. We also grouped SPEGs that appeared at a consistent DM but different times together as these groups would have a higher probability of having an astrophysical origin.

4. We extended the feature set to characterise both individual SPEGs and the SPEG groups, and as in previous study [26] used supervised machine learning algorithms in combination with treatment for imbalanced data to automatically classify SPEGs as pulsars and non-pulsars.

5. Last but not least, we calculated the probabilities of a group of (three to five) purely randomly distributed single-pulse events that were found to have an underlying periodicity. Additionally, we confirmed the astrophysical origin of dim SPEG candidates by searching for an underlying periodicity among them, which helped to differentiate dim SPEGs/pulses from RFI and noise SPEGs. 
The code of SPEGID was implemented in PYTHON and made available in the Astrophysics Source Code Library. ${ }^{2}$

\subsection{Single-pulse Events}

The single-pulse search data set used in this research was processed by the PRESTO code single_pulse_search.py [89], in which the spacing of the trial DM values (that were used to dedisperse the data) increases at higher DMs. After matched filtering, only singlepulse events with $\mathrm{S} / \mathrm{N}$ above 5 were recorded. Figure 4.2 shows a typical astrophysical pulse detected as a group of related single-pulse events. As it can be seen in the top left zoomed DM versus time subplot, there is a slight variance in time between neighboring single-pulse events. For this reason, we must also understand the expected spread of single-pulse events in time in order to group them correctly. In fact, for two adjacent single-pulse events separated by one trial DM step, this difference in time is typically one time sample, given the way that DM channels are spaced and downsampling factors are chosen (see Section 4.3.1). Meanwhile, as shown in the top right S/N versus DM subplot, the observed $\mathrm{S} / \mathrm{N}$ of the pulse decreases as the DM offset $(\delta \mathrm{DM})$ from the true DM increases. This relation is characterised by Equation (4.1) [19]:

$$
\frac{\mathrm{S}(\delta \mathrm{DM})}{\mathrm{S}}=\frac{\sqrt{\pi}}{2} \zeta^{-1} \operatorname{erf} \zeta
$$

where $\mathrm{S}(\delta \mathrm{DM}) / \mathrm{S}$ is the ratio of observed $\mathrm{S} / \mathrm{N}$ to true peak $\mathrm{S} / \mathrm{N}$, erf is the error function, and $\zeta$ is given by

$$
\zeta=6.91 \times 10^{-3} \delta \mathrm{DM} \frac{\Delta \nu}{W \nu^{3}} .
$$

In Equation (4.2), $\Delta \nu$ is the total bandwidth in MHz, $W$ is the pulse width (FWHM) in milliseconds, and $\nu$ is the central observing frequency in GHz. Note that equations (4.1) and (4.2) assume a Gaussian pulse. With these two equations, given the $\mathrm{S} / \mathrm{N}$ and the width $W$ of an astrophysical pulse, we can calculate the expected threshold for the DM offset $(\delta \mathrm{DM})$ that will make the observed $\mathrm{S} / \mathrm{N}$ decrease to the noise level (i.e., $\mathrm{S} / \mathrm{N}=$ 5). An example is shown in the top right subplot in Figure 4.2. In this subplot, we show both the expected and fitted $\mathrm{S} / \mathrm{N}$ decline. In both cases, the DM at which the $\mathrm{S} / \mathrm{N}$ peaks is considered as the true DM. We calculated the expected $\mathrm{S} / \mathrm{N}$ decline using the peak $\mathrm{S} / \mathrm{N}$ and the pulse width obtained from matched filtering, which is a close approximation to the actual pulse width. On the other hand, to calculate the fitted $\mathrm{S} / \mathrm{N}$ decline, we

\footnotetext{
${ }^{2}$ http://ascl.net/1807.014http://ascl.net/1807.014
} 

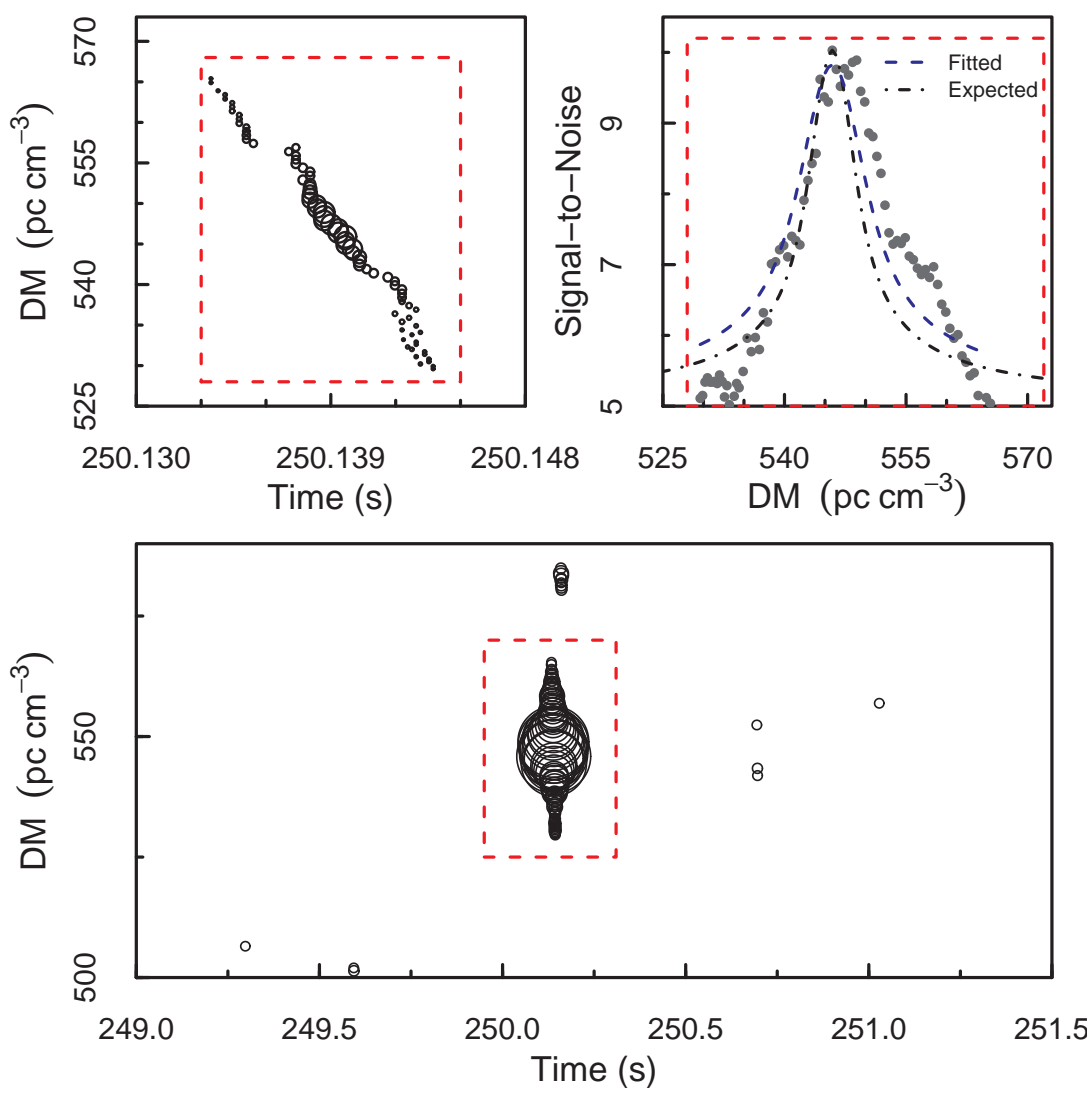

Figure 4.2: A typical astrophysical pulse detected as of a group of single-pulse events. The bottom and the top left subplots show its appearance in the DM versus time space, and the top right subplot show its $\mathrm{S} / \mathrm{N}$ versus $\mathrm{DM}$ curve. Note that in both the top left and the bottom subplots, the single-pulse events are both plotted proportionally to $\mathrm{S} / \mathrm{N}$ but with different scaling to show the single-pulse events more clearly in the top left subplot. In the top right subplot, we also show both the fitted and expected $\mathrm{S} / \mathrm{N}$ decline that are caused by DM offset. Note that they are both fairly close to the observed $\mathrm{S} / \mathrm{N}$ decline, and to each other as well. 
first used a non-linear least squares (NLS) regression to obtain the fitted peak S/N and $W$ of the pulse and then calculated the fitted $\mathrm{S} / \mathrm{N}$ decline using the same equations. It can be seen that the expected $\mathrm{S} / \mathrm{N}$ decline is fairly close to the fitted values even though the observed peak $\mathrm{S} / \mathrm{N}$ and the approximate pulse width were used. Furthermore, by assuming a time drift of one sample for each DM step, we can also find the expected time extent of this pulse. In fact, we use this idea to merge clusters that likely originate from the same pulse, as described in Section 4.3.2.

\subsection{Identification of SPEGs}

\subsubsection{DBSCAN Clustering}

Clustering is an unsupervised learning method that involves grouping data objects with similar properties together [45]. It is very useful in finding previously unknown groups in a data set. The similarity (and therefore, dissimilarity) between data objects is usually determined by the distance measure derived from their attributes. Clustering algorithms are divided into two categories: partitioning (non-hierarchical) and hierarchical. While partitioning methods separate data objects into a number of clusters, hierarchical methods link the cluster pairs successively and thus form a nested hierarchy among clusters [90]. Since related single-pulse events are typically close in DM and time, partitioning clustering methods can be used to identify pulse candidates and separate them from each other. Specifically, we applied DBSCAN clustering [32] in the DM versus time space and thus partitioned the single-pulse events into signals and noise by measuring their density. We demonstrate below how DBSCAN clustering works on single-pulse events using one astrophysical pulse from Figure 4.3 (which displays part of the diagnostic plot shown in Figure 2.6) as an example. We used scikit-learn's implementation of DBSCAN clustering in PYTHON [83].

DBSCAN clustering requires two parameters: the density threshold (MinPts) of a core object and the radius $(\epsilon)$ of its neighborhood. If an object has at least MinPts of adjacent objects in its $\epsilon$-neighborhood, then this object is considered a core object. Core objects and their neighborhood are considered as dense regions and form clusters. If the dense regions of two core objects overlap, they are joined together to form one single cluster. On the other hand, objects that are neither core objects themselves, nor fall into the neighborhood of any core object, are classified as noise [32]. It should be emphasized that although the shape of the neighborhood is controlled by the distance 

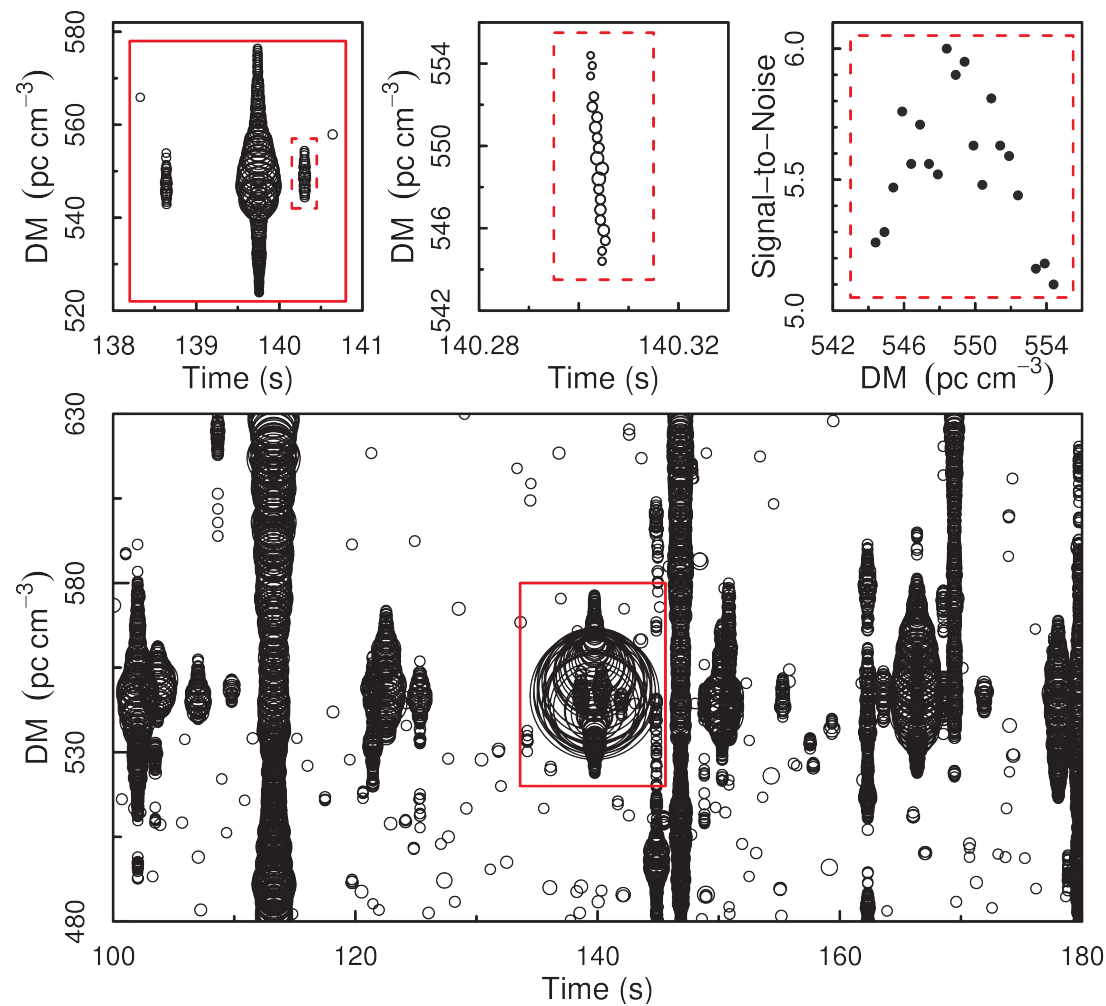

Figure 4.3: A zoomed-in part of the diagnostic plot shown in Figure 2.6. The region surrounded by the solid rectangle in the bottom subplot is further enlarged to show the details in the top left subplot, in which three clusters of single-pulse events can be seen. The smaller cluster on the right (surrounded by a dashed rectangle) is zoomed in and shown in the top middle and the top right subplots. This cluster is used to demonstrate DBSCAN clustering in Figure 4.4.

metric between objects (for example, if Euclidean distance is used, the shape of the neighborhood will be circular), the shape of the clusters (as amalgamated core points with their neighborhood) can be arbitrary.

We chose to use Euclidean distance in our application, with time and DM channel indices as our dimensionless units (see Figure 4.4). (Even though other distance metrics could be used, they do not seem to have any particular advantage). Figure 4.4 shows the small cluster of single-pulse events from the top left subplot of Figure 4.3. A closer examination shows that for two adjacent single-pulse events, as the DM increases by one DM spacing, the time decreases by approximately one sample. This is an artefact of the dedispersion in which higher DM channels have later start times; these pulses actually arrive at the same time in different DM channels. Our distance metric will allow for 
these slopes in the DM versus time plane.

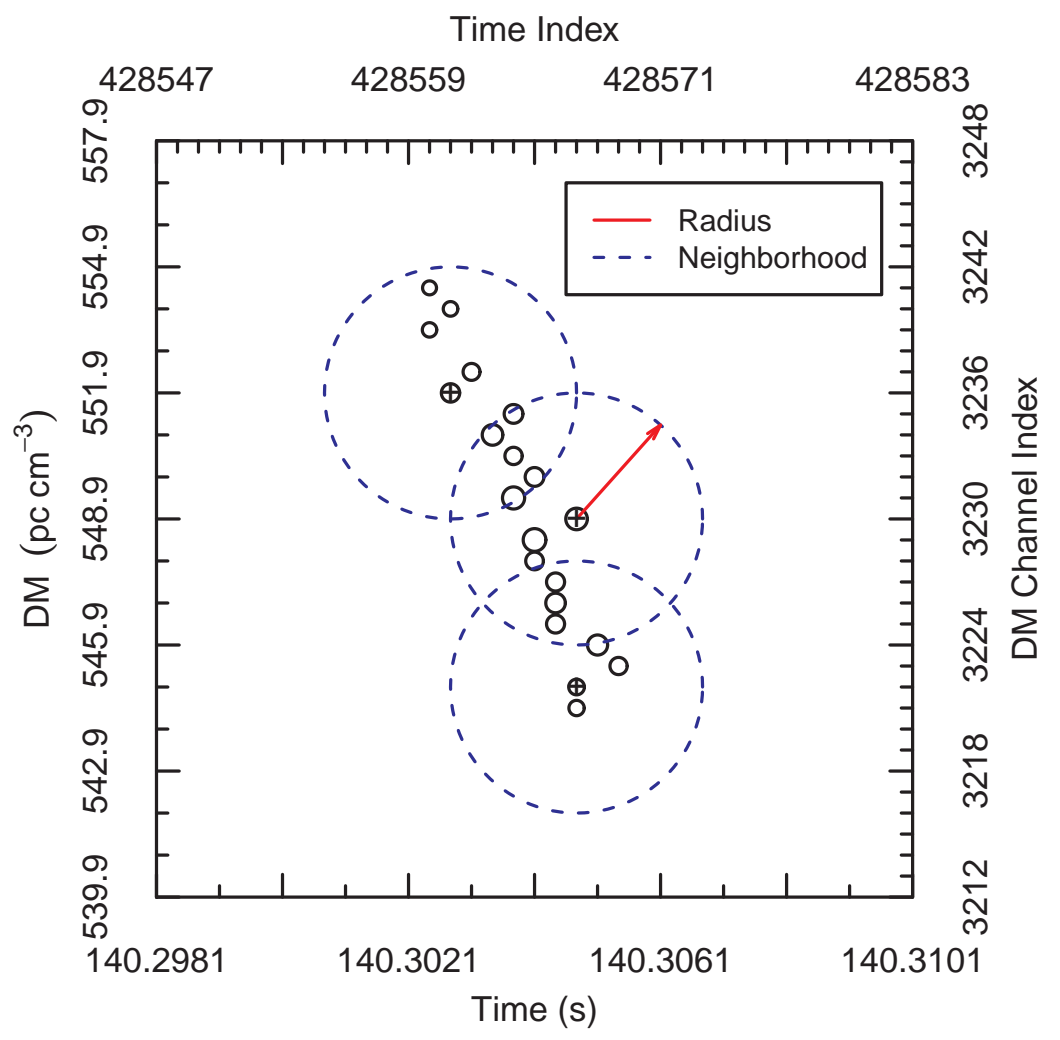

Figure 4.4: An example of DBSCAN clustering applied on the cluster from Figure 4.3. Note that instead of the actual time and DM, time and DM channel indices were used to calculate the distance between single-pulse events. In this example, all single-pulse events are core objects in DBSCAN clustering and the radius of their neighborhood is small enough to avoid including any noise event. Because their neighborhood areas overlap with one another, all single-pulse events are joined together to form one single cluster. It can also be seen for two adjacent single-pulse events, a unit increase in DM channel index roughly corresponds to a unit decrease of time index.

The effect of DBSCAN clustering on single-pulse events is shown in Figure 4.4, using a density threshold MinPts $=5$ and a radius $\epsilon=6$ for the neighborhood. It can be seen that the single-pulses events successfully form a single cluster without including any noise event. Next, we describe the reasons behind choosing these particular values for the parameters MinPts and $\epsilon$.

MinPts value selection. Unlike the bright, wide pulses which are detectable in many DM channels, narrow and/or dim pulses can only be detected in a few DM channels 
(especially when the DM spacing is large). In extreme cases, we have observed pulses having less than $2 \mathrm{pc} \mathrm{cm}^{-3}$ span in the DM domain. Such pulses would be missed by DBSCAN clustering if the DM spacing is large. One way to solve this is to use denser DM spacing in the data processing step of the pipeline. In the absence of that, our current best strategy is to choose a small MinPts value, so that we can detect most pulses when DM spacing is small and as many pulses as possible when DM spacing becomes larger. For this reason, we used 5 as the optimal value for MinPts so we could identity as many narrow and/or dim pulses as possible. Note that it would be difficult to confirm the expected $\mathrm{S} / \mathrm{N}$ versus $\mathrm{DM}$ shape with less than five events.

$\epsilon$ value selection. Ideally speaking, if the single-pulse events form a diagonal line in the DM versus time space, we should be able to find 5 single-pulse events within a $2 \sqrt{2}$ radius of a core object. However, because the same DM and time spacing was used for a range of DMs, the 1-to-1 relation between time and DM channel indices is only approximate for the entire DM range. As shown in Figure 4.4, single-pulse events oftentimes do not form a diagonal line. Therefore, it is often observed that the maximum time drift between two adjacent single-pulse events is much larger than one time sample, especially in bright and wide pulses that are made of many single-pulse events. Due to possible large time difference and/or the occasional non-detection of single-pulse events in certain DM channels, we found that $\epsilon=6$ was optimal for discriminating pulses from other pulses and/or noise, while still allowing for larger than expected distances.

Generally speaking, the parameters of DBSCAN clustering MinPts and $\epsilon$ can be modified to suit surveys with different observational setups and/or different research goals. For example, if the predetermined DM spacing is really dense (or in a less likely case, if narrow pulses are not of interest), the density threshold can be increased in order to filter out noise clusters that are made up of less than MinPts single-pulse events. If a larger MinPts is selected, a larger radius $\epsilon$ should be used accordingly.

For beams in which consistent RFI is detected during the observation duration or many pulses from a very bright pulsar are detected, many single-pulse events will be generated. When DBSCAN clustering is applied to such beams, a large number of single-pulse event clusters will usually be formed. Investigating all these clusters not only requires significant computing power, it is also unnecessary. This is because in the former case, single-pulse event clusters with low maximum $\mathrm{S} / \mathrm{N}$ are more likely from RFI than real astrophysical signals, whereas in the latter case, the identification of those bright pulses is sufficient for the detection of the pulsar. In other words, when the cluster 
density is high, we only need to consider those relatively bright clusters. As a result, after running DBSCAN clustering, we calculated the cluster density as the number of clusters divided by the product of the number of trial DM channels $\left(\mathrm{N}_{\mathrm{DM}}\right)$ and the observation duration $\left(t_{\mathrm{obs}}\right)$ :

$$
\text { ClusterDensity }=\frac{\mathrm{N}_{\text {cluster }}}{\mathrm{N}_{\mathrm{DM}} \times t_{\mathrm{obs}}} .
$$

Note that the cluster density of a beam is normalised over the observation duration hence it is independent of the observation duration. Such statement could not be made for the number of clusters as it is not uncommon for two observations to have different durations (even within the same survey). Specifically, we define the clusters to be bright or $\operatorname{dim}$ as follows:

1. If the cluster density is less than 0.01 per channel per second, all clusters (with maximum $\mathrm{S} / \mathrm{N} \geq 5$ ) are further investigated. Clusters with maximum $\mathrm{S} / \mathrm{N} \geq 6$ are considered as bright clusters, and the rest are considered as dim clusters;

2. If the cluster density is between 0.01 and 0.02 per channel per second, only clusters with maximum $\mathrm{S} / \mathrm{N} \geq 5.5$ are further investigated. Clusters with maximum $\mathrm{S} / \mathrm{N}$ $\geq 6.5$ are considered as bright clusters, and the rest are considered as dim clusters;

3. If the cluster density is greater than 0.02 per channel per second, only clusters with maximum $\mathrm{S} / \mathrm{N} \geq 6$ are further investigated. Clusters with maximum $\mathrm{S} / \mathrm{N} \geq 7$ are considered as bright clusters, and the rest are considered as dim clusters.

Considering that a typical PALFA observation has an observation length of $268 \mathrm{~s}$, and the number of trial DM channels is often greater than 5,000, we only ignore single-pulse event clusters with peak $\mathrm{S} / \mathrm{N}<5.5$ when the total number of clusters exceeds 13,400 $(268 \times 5,000 \times 0.01)$, and clusters with peak $\mathrm{S} / \mathrm{N}<6$ when the total number of clusters exceeds $26,800(268 \times 5,000 \times 0.02)$. Our preliminary experimentation showed that: (i) For the majority of beams, usually much fewer clusters were found, therefore all clusters would be further investigated; (ii) For beams in which consistent RFI or very bright pulsars were observed, ignoring the dim clusters helped to decrease the running time of our algorithms significantly (which was necessary due to the large number of beams in our data set), yet minimised the possibility of missing potential pulsar discoveries. Note that because cluster density is independent of the observation duration and we selected fairly conservative threshold values for the sake of reasonable computation time, Equation (4.3) and the threshold values given above can be used for other surveys with different observation lengths. 
The definition of bright clusters will be referred to later, when clusters are merged into SPEGs in Section 4.3.2. SPEGs that contain at least one bright cluster will be considered as bright SPEGs and used to form SPEG groups in Section 4.3.4.

\subsubsection{Merging Clusters Vertically into SPEGs}

By applying DBSCAN clustering, we are able to separate pulses from other pulses and/or noise. However, instead of grouping all single-pulse events from the same astrophysical pulse into one SPEG, DBSCAN clustering (with the parameters given in Section 4.3.1) sometimes resulted in "broken" SPEGs (i.e., single-pulse events from one astrophysical pulse were grouped into multiple clusters). This was because of the unusually large distance between adjacent single-pulse events, and is mainly due to the following two reasons.

The first reason that can cause a larger than expected distance is clipping. Often, in pulsar search algorithms, samples with very high intensity are "clipped", or replaced by the median values of the time series. This can affect both bright pulses and RFI, and is used by the PRESTO prepsubband command used in the PALFA pipeline. As a result, we often observed two different types of pulses in single-pulse search output: regular (non-clipped) pulses (see Figure 4.2) and clipped pulses (shown in Figure 4.5). As can be seen in Figure 4.5, clipping has removed the brightest single-pulse events from the $\mathrm{DM}$ versus time subplot and, correspondingly, peaks from the $\mathrm{S} / \mathrm{N}$ versus $\mathrm{DM}$ subplot, which leads to "broken" astrophysical pulses. If the broken parts of a pulse are not merged together, no peak-like shape would be detected in the S/N versus DM subplot and the signal would be interpreted as RFI. This is especially problematic for FRBs or RRATs that emit a small number of bright pulses during an observation.

The second reason is the unusually large time drift between two adjacent single-pulse events, which is commonly observed in bright and wide pulses that are made of many single-pulse events. This can cause these pulses to break into multiple clusters during DBSCAN clustering, as shown in Figure 4.6.

Theoretically, using a much larger radius in DBSCAN clustering can solve the problem of "broken" SPEGs. However, one of the requirements set for our approach was that it should be able to separate pulses even when the time difference between them is small. Thus, instead of using a much larger radius in DBSCAN clustering that would inevitably group such pulses together, we introduced an extra step of merging the clusters vertically after DBSCAN clustering was completed. 

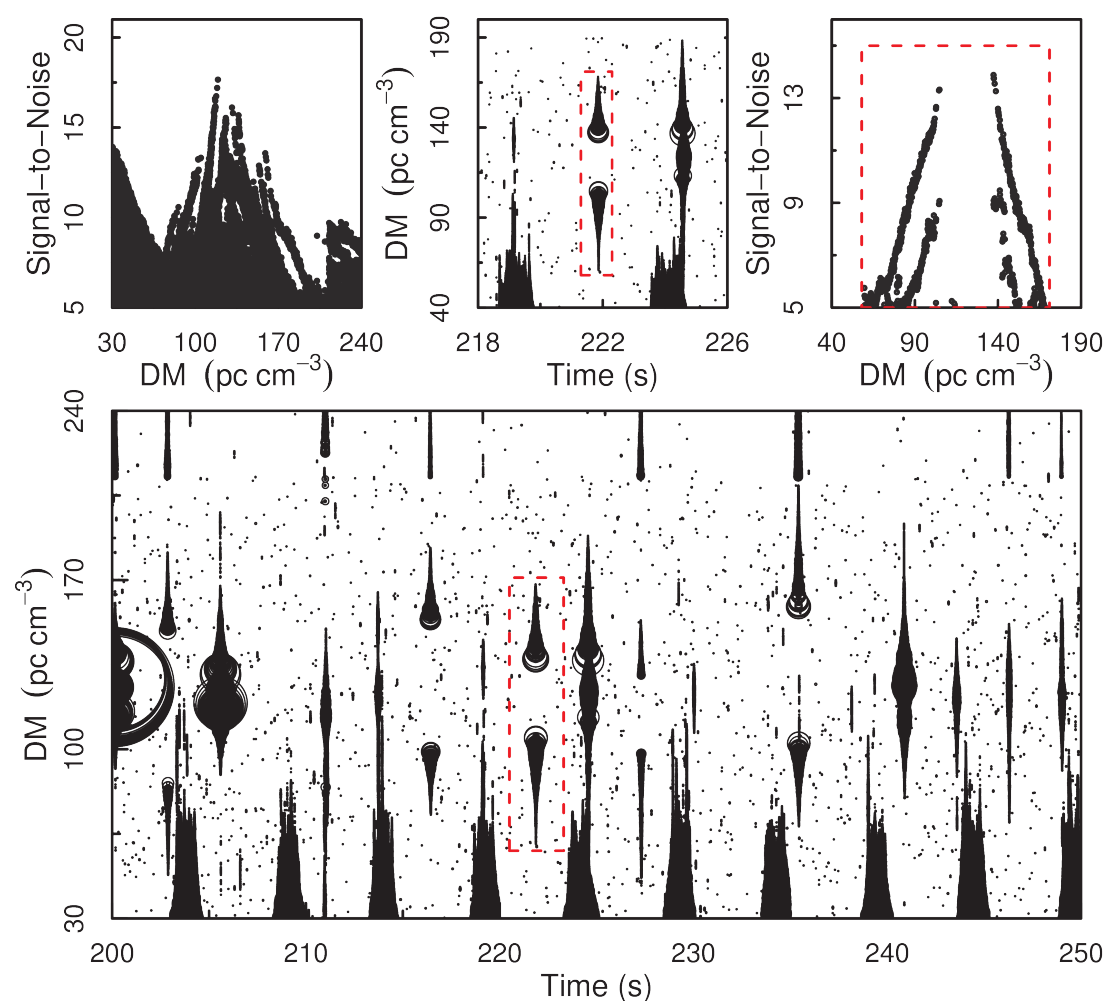

Figure 4.5: Known pulsar J1910+0714 detected on MJD 56663 in the PALFA survey. Note that there are many clipped pulses. The top left subplot and the bottom subplot show the $\mathrm{S} / \mathrm{N}$ versus $\mathrm{DM}$ space, and the DM versus time space of this whole section respectively; and the other two subplots show those regions around the clipped pulse of interest that is surrounded by the red dashed line rectangle.

As described in Section 4.2, given the peak $\mathrm{S} / \mathrm{N}$ and width $W$ of a pulse, we can calculate the expected maximum DM offset that would cause the observed $\mathrm{S} / \mathrm{N}$ to fall below 5, and hence the expected extent of an astrophysical pulse in DM and time. Correspondingly, the merging of clusters can be conducted as follows. After DBSCAN clustering, if a dimmer cluster is identified within the expected DM and time span of a brighter cluster, then the dimmer cluster needs to be merged with the brighter one. The merged cluster as a whole is considered as detection of a single pulse. This suggests that merging should start from the brightest cluster and continue in descending order of maximum $\mathrm{S} / \mathrm{N}$. However, there are several additional challenges that needed to be addressed, which are listed below. While the first three challenges mainly relate to non-clipped/regular wide pulses, the last two only relate to clipped pulses.

First of all, for most non-clipped pulses that have a narrow DM extent, merging 
is unnecessary because extremely large time drift between adjacent single-pulse events is unlikely to be observed among them. Therefore, using $\epsilon=6$ in DBSCAN clustering provides enough robustness for slightly larger time drift and the occasional non-detection of single-pulse events in certain DM channels.

Second, while calculating the expected $\mathrm{S} / \mathrm{N}$ decline is fast, calculating the fitted $\mathrm{S} / \mathrm{N}$ decline greatly increases the complexity and run time of our algorithm. This is because to find the accurate DM and time span of an astrophysical pulse, parameters including the true peak $\mathrm{S} / \mathrm{N}$, the width, and DM all have to be first obtained through NLS regression of equations (4.1) and (4.2) before they can be plugged into the same formulas to calculate the fitted $\mathrm{S} / \mathrm{N}$ decline. On the other hand, for most bright and wide pulses that are detected as many single-pulse events, the difference between the expected $\mathrm{S} / \mathrm{N}$ decline and the fitted $\mathrm{S} / \mathrm{N}$ decline is fairly small, as for the example shown Figure 4.2. Furthermore, in case of a pulse that forms multiple single-pulse event clusters in DBSCAN clustering (as the one shown in Figure 4.6), theoretically, the SPEG may
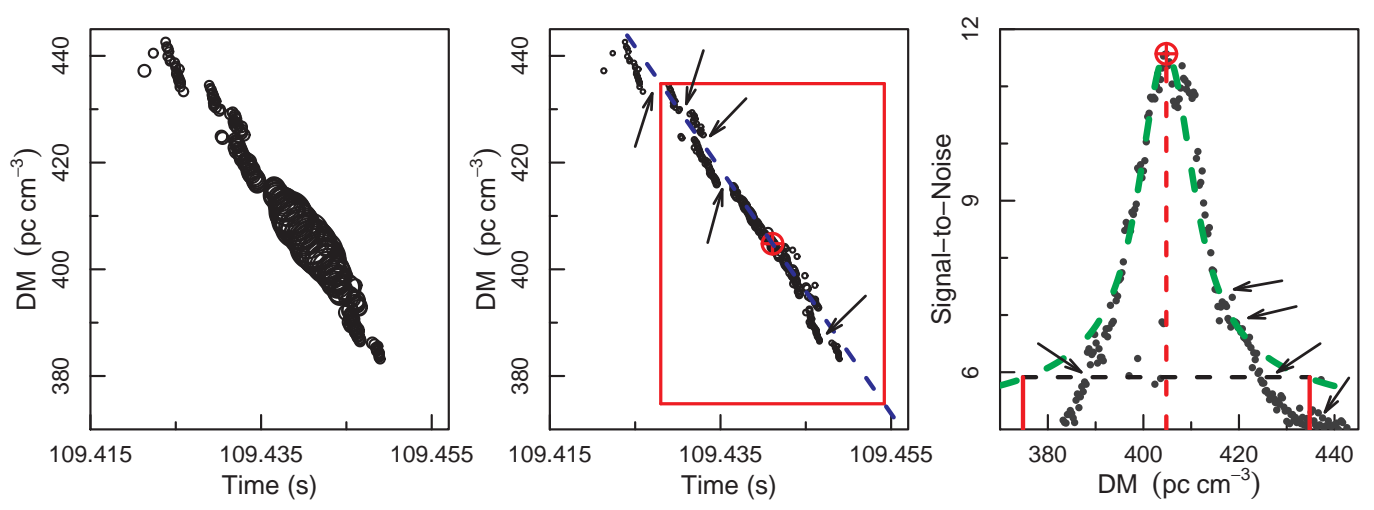

Figure 4.6: Merging the clusters in a non-clipped pulse. In the left and middle subplots, the single-pulse events are both plotted proportionally to $\mathrm{S} / \mathrm{N}$ but with different scaling in order to show the distance between single-pulse events more clearly. In the middle subplot, the red circle shows the brightest single-pulse event, and the red solid rectangle shows the expected DM and time span of the pulse. In the right subplot, the red circle shows the brightest single-pulse event and the red dashed line shows its DM, and the blue dashed line shows the expected (i.e., not fitted) S/N decline. The black horizontal dashed segment shows the $\mathrm{S} / \mathrm{N}$ threshold $\left(\mathrm{S}_{\mathrm{th}}\right)$ calculated from Equation (4.4). The two red vertical segments show the limit of the DM offset where the $\mathrm{S} / \mathrm{N}$ decreases below $\mathrm{S}_{\text {th }}$. The arrows show where the pulse breaks into several clusters in DBSCAN clustering because a small radius is used. 
break at any part, thus it is unlikely that the fitted peak $\mathrm{S} / \mathrm{N}$, width, and $\mathrm{DM}$ would be significantly more accurate than the observed values. If so, the fitted $\mathrm{S} / \mathrm{N}$ decline would not be better than its expected counterpart either. Therefore, having in mind the large number of beams in our full data set, we only calculated the expected $\mathrm{S} / \mathrm{N}$ decline and used it to find the expected DM and time span of the pulse.

Third, in practice, using 5 as the threshold $\left(\mathrm{S}_{\text {th }}\right)$ for the $\mathrm{S} / \mathrm{N}$ decline more often than not results in an unnecessarily large DM offset. This is mainly because when the $\mathrm{S} / \mathrm{N}$ decreases closely to the noise level (i.e., $\mathrm{S} / \mathrm{N}=5$ ) due to large DM offset, the observed $\mathrm{S} / \mathrm{N}$ begins to decrease much faster than the expected $\mathrm{S} / \mathrm{N}$ decline, as shown in the right subplot in Figure 4.6. This is true even for the fitted $\mathrm{S} / \mathrm{N}$ decline (see the top right subplot in Figure 4.2). Such difference can be even larger for brighter pulses. Therefore, instead of using a fixed value of 5 , we derived the following empirical formula (through trial and error) to calculate a dynamic threshold $\mathrm{S}_{\mathrm{th}}$ :

$$
\mathrm{S}_{\mathrm{th}}=0.4 \log _{2}(\text { peak } \mathrm{S} / \mathrm{N})+4.5
$$

This dynamic threshold meets the following criteria: (i) $\mathrm{S}_{\text {th }}$ should be smaller than peak $\mathrm{S} / \mathrm{N}$. For any peak $\mathrm{S} / \mathrm{N} \geq 5.49$, Equation (4.4) always returns $\mathrm{S}_{\text {th }}$ that is lower than peak $\mathrm{S} / \mathrm{N}$. Clusters with peak $\mathrm{S} / \mathrm{N}<5.49$ are rarely the starting point of merging because merging is not needed for dim pulses as they are unlikely to break into multiple clusters in DBSCAN clustering, or such clusters are made of single-pulse events that are detected at large DM offsets and therefore should be merged with brighter clusters detected at smaller DM offsets; (ii) $\mathrm{S}_{\text {th }}$ should be greater than 5 in oder to make the calculated DM and time span become smaller, and closer to the actual span; (iii) $\mathrm{S}_{\text {th }}$ should be higher for brighter pulses (i.e., higher peak $\mathrm{S} / \mathrm{N}$ ), thus it should increase as peak $\mathrm{S} / \mathrm{N}$ increases but at a much slower pace, which explains the introduction of the non-linear log relation. The parameters in Equation (4.4) were selected by manually varying the values until they worked well for the majority of non-clipped pulses in our preliminary exploration.

An example of using the threshold obtained from Equation (4.4) to calculate the DM and time span is shown in Figure 4.6. As can be seen in the middle subplot, the calculated DM and time span is smaller in contrast to using $\mathrm{S} / \mathrm{N}=5$ as the threshold. In the right subplot in Figure 4.6, a small section of the pulse in the high DM region is not merged and forms a separate SPEG because the observed S/N decline has a longer tail in the high DM region. Nevertheless, the merged SPEG does include the majority of single-pulse events derived from the pulse, which is good enough for the subsequent steps 
including peak scoring, feature extraction, and automatic classification. Since the S/N of the remaining section monotonically decreases as DM increases, no peak-like shape would be found in this single-pulse event cluster, and therefore it would be filtered in the next step in Section 4.3.3. Note that $\mathrm{S}_{\text {th }}$ is only used to calculate a more accurate expected DM range in the merging step. It does not serve as a criteria to further filter any cluster. It is also worth mentioning that, for really bright pulses, the threshold calculated by Equation (4.4) can be a little conservative (i.e., the calculated DM and time span is smaller than the actual span) that all clusters originating from the same pulse may not be merged by the one containing the brightest single-pulse event. Nevertheless, this is not a problem because the remaining clusters will appear and merge like a clipped pulse, which is described next. Consequently, in SPEGID, we included one extra step of removing duplicate SPEGs (SPEGs having the same brightest single-pulse event) after merging.

The fourth challenge is due to the fact that, in a clipped pulse, the peak $\mathrm{S} / \mathrm{N}$ and its $\mathrm{DM}$, as well as the pulse width, are all unknown, because the brightest single-pulse event is missing. As mentioned earlier, calculating the fitted $\mathrm{S} / \mathrm{N}$ decline would significantly increase the complexity and run time of our algorithm. Without fitting, the best way to calculate an approximate DM and time span for the clipped pulse is to use the attributes of the brightest single-pulse event of the remaining brightest cluster. Because this singlepulse event is the closest to the actual clipped peak event, the DM and time span calculated from the former would also be close to that from the latter. However, the S/N decline calculated from the remaining brightest single-pulse event (using Equation (4.4)) tends to decrease too fast towards the side where the pulse was clipped, and too slow towards the other side. This constitutes the last (i.e., fifth) challenge of the merging step.

Finally, when a pulse is clipped, clipping causes the cluster containing the remaining brightest single-pulse event to end abruptly in the $\mathrm{S} / \mathrm{N}$ versus DM space. To make the calculated DM and time span closer to the actual span, we adjusted Equation (4.4) to further extend the DM and time extents towards the clipped side and shorten them towards the other side, which is equivalent to shifting the calculated DM and time span towards the clipped side. Specifically, for a clipped pulse, $S_{\text {th }}$ is calculated using the following Equation:

$$
\mathrm{S}_{\mathrm{th}}= \begin{cases}\mathrm{S}_{\mathrm{th}}=0.35 \log _{2}(\text { peak } \mathrm{S} / \mathrm{N})+4.5 & \text { on the clipped side } \\ \mathrm{S}_{\mathrm{th}}=0.50 \log _{2}(\text { peak } \mathrm{S} / \mathrm{N})+4.5 & \text { on the other side }\end{cases}
$$


As in Equation (4.4), the parameters in Equation (4.5) were selected by a trial and error process until they worked well for the majority of clipped pulses in our preliminary exploration. Figure 4.7 shows an example of merging a clipped pulse. As can be seen in the middle subplot, the clusters are successfully merged to form a single SPEG. The right subplot shows that using asymmetric DM and time extents is necessary because the SPEG is no longer symmetric to the hypothetical peak.
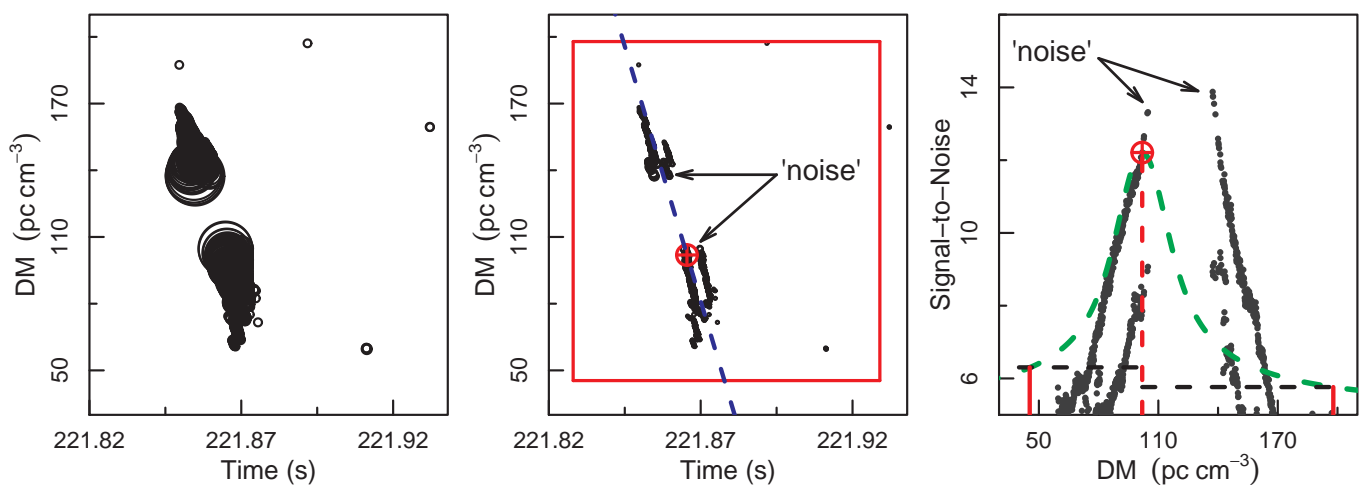

Figure 4.7: Merging the clipped pulse from Figure 4.5. The red rectangle in the middle subplot shows the calculated DM and time ranges. Compared with the left subplot, the single-pulse events in the middle subplot are plotted using a smaller scaling to show more details. In the middle and right subplots, the red circle shows the single-pulse event with the maximum $\mathrm{S} / \mathrm{N}$ among all clusters returned by DBSCAN clustering, from which the calculated DM and time span of the pulse is calculated and shown as the red solid rectangle in the middle subplot. Note that the hypothetical peak event is not the brightest event in the right subplot as all the brighter events are excluded from DBSCAN clustering (i.e., there are less than MinPts events in their $\epsilon$ neighborhood, and they don't fall into the $\epsilon$ neighborhood of any other core point) and are classified as "noise" (they are not really noise events). In the right subplot, the blue dashed line is the expected S/N decline calculated from the hypothetical peak. The two black horizontal dashed segments show the $\mathrm{S} / \mathrm{N}$ threshold $\left(\mathrm{S}_{\text {th }}\right)$ calculated from Equation (4.5). The two red vertical segments shows the limit of the DM offset where the $\mathrm{S} / \mathrm{N}$ decreases below $\mathrm{S}_{\text {th }}$. By using different $\mathrm{S}_{\text {th }}$ obtained on the two sides, the expected DM and time span is closer to the actual span.

Although we only demonstrate the effect of merging clusters into SPEGs using data from the PALFA survey, equations (4.1), (4.2), (4.4) and (4.5) are applicable to data from other surveys with no (or minimal) changes of the parameters' values. Specifically, 
in equations (4.1) and (4.2), the central observing frequency $(\nu)$ and the total bandwidth $(\Delta \nu)$ are observational setup parameters specific to each survey, whereas the DM offset $(\delta \mathrm{DM})$, the pulse width $(W)$ and observed $\mathrm{S} / \mathrm{N}$ are all obtained in data processing. Therefore, once the observation parameters are given, equations (4.1) and (4.2) can be applied to data collected in any survey. Note, however, that equations (4.1) and (4.2) only describe the expected (symmetric, bell-shaped) S/N versus DM curve of astrophysical pulses [19]. As this is generally not true for RFI, the calculated DM and time span of RFI may not be as accurate. With respect to equations (4.4) and (4.5), the criteria we used to formulate these equations should be followed regardless of the observation parameters. It is important to mention that we tested the equations (4.1), (4.2), (4.4) and (4.5) with no modification (except for the values of $\nu$ and $\Delta \nu$ ) on data from the Green Bank Telescope (GBT) Drift-scan survey [50], and they turned out to work equally well.

Notice that after merging the clusters vertically, we removed SPEGs that peak at $\mathrm{DM}<=2 \mathrm{pc} \mathrm{cm}-3$, which is a general practice to remove RFI [50]. As illustrated in Figs. 4.6 and 4.7, the combination of a small neighborhood radius and merging algorithm successfully identified astrophysical pulses as SPEGs, even if they are broken in the DM versus time space. Therefore, in the rest of the proposal, we use "SPEG" and "pulse" interchangeably to refer to both the merged SPEG as a whole and the single-pulse events within. Furthermore, as shown in Figure 4.8, our approach effectively eliminates noise that was made up of less than 5 single-pulse events.

\subsubsection{Calculating the Peak Score for SPEGs}

Through DBSCAN clustering of single-pulse events and merging clusters vertically, we obtained a list of SPEGs, among which astrophysical pulses can be identified. According to Equation (4.2), the $\mathrm{S} / \mathrm{N}$ values of astrophysical pulses peak at the true $\mathrm{DM}$ and fall off as the DM offset increases. Searching for this signature within SPEGs can help to discriminate astrophysical pulses against noise and RFI. In related works, different peak identification algorithms were proposed to identify such trends [50, 26]. However, the main challenge of peak identification lies in the variety of the $\mathrm{S} / \mathrm{N}$ versus DM curves of the pulses (due to varied pulse widths and brightness). Our peak scoring algorithm is inspired by the Gaussian shape of the $\mathrm{S} / \mathrm{N}$ versus DM curve, and two other peak identification algorithms $[50,26]$. A unique characteristic of our algorithm is that it accounts for the fact that different DM spacings are used in different DM ranges, which has been 

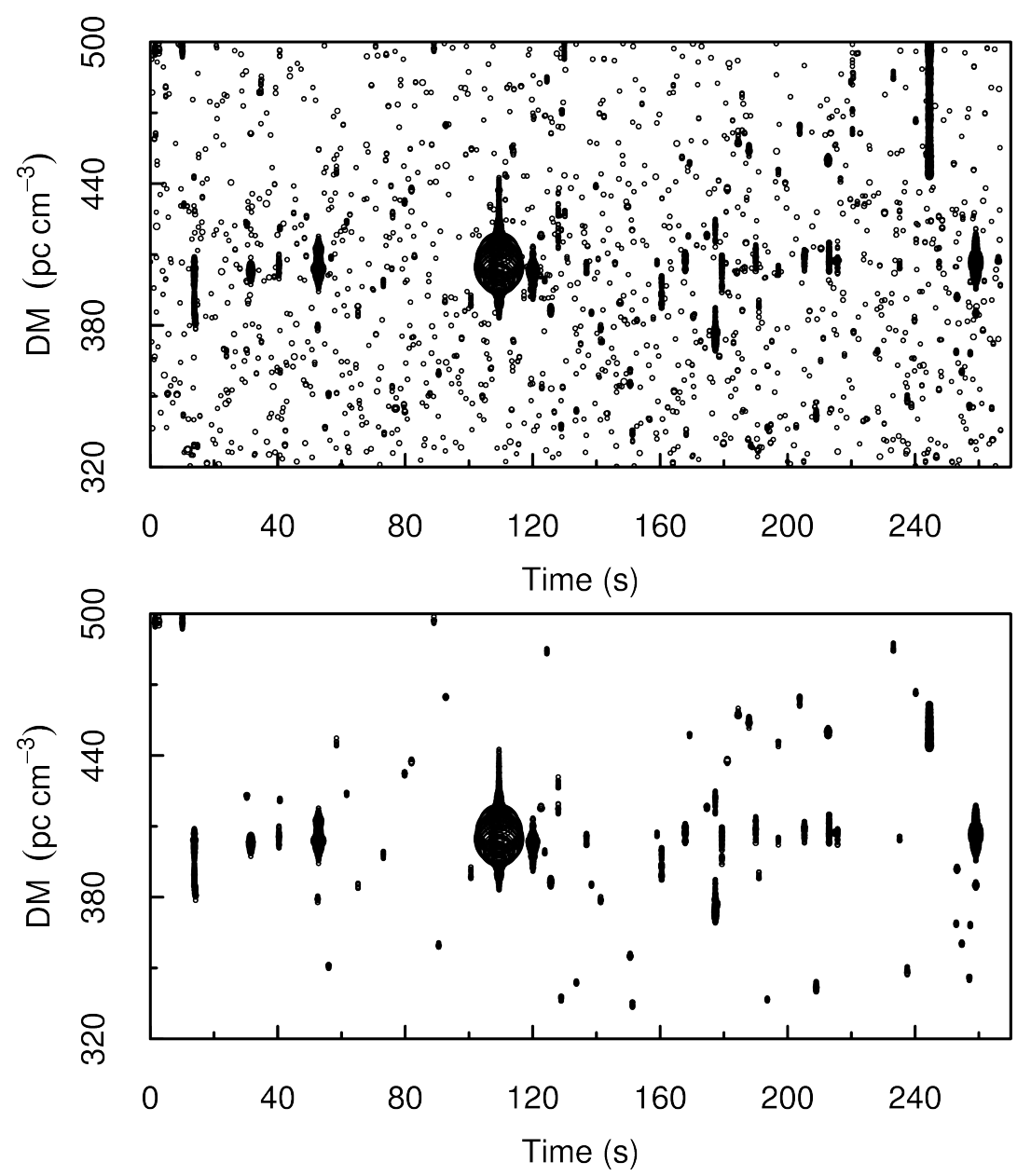

Figure 4.8: The DM versus time plot for pulsar J1901+02 detected in the PALFA survey. The top subplot shows all single-pulse events within the DM and time range, while the bottom subplot only shows those single-pulse events that belong to SPEGs identified by DBSCAN clustering and merging of clusters. The comparison between the top and bottom subplots illustrates the denoising effect of these two steps.

ignored so far. This is achieved by making the slope threshold dependent on the DM spacing, which proves to be effective in finding dim and/or wide pulses. Furthermore, our peak scoring algorithm is not only capable of identifying the peak in $\mathrm{S} / \mathrm{N}$ versus DM curves of various shapes, it also returns a score that characterises the shape of a curve. The peak score conveys additional information about the relative "steepness" of the $\mathrm{S} / \mathrm{N}$ versus DM curve, which is used as one of the features in the automatic classification step (see Section 4.5.1).

Our peak scoring algorithm calculates the peak score for each SPEG in the following way: 
1. If the brightest single-pulse event within the SPEG does not have any neighboring event within 5 adjacent DM channels on both sides, the SPEG is then considered as clipped, and it is treated differently from a non-clipped SPEG;

2. Because equations (4.1) and (4.2) predict a symmetric S/N versus DM curve for astrophysical pulses, we propose two symmetry indices, $\mathrm{SI}_{\mathrm{DM}}$ and $\mathrm{SI}_{\mathrm{S} / \mathrm{N}}$, to characterise the symmetry of the S/N versus DM curve of an SPEG. These two symmetry indices are used as two of the many features that help to distinguish astrophysical SPEGs from non-astrophysical SPEGs. $\mathrm{SI}_{\mathrm{DM}}$ and $\mathrm{SI}_{\mathrm{S} / \mathrm{N}}$ are calculated as follows:

$$
\mathrm{SI}_{\mathrm{DM}}=\frac{\min (\operatorname{maxDM}-\text { peakDM, peakDM }-\operatorname{minDM})}{\max (\operatorname{maxDM}-\text { peakDM, peakDM }-\operatorname{minDM})},
$$

where maxDM is the maximum DM of the SPEG, peakDM is the DM of the brightest single-pulse event and minDM is the minimum DM of the SPEG, and

$$
\mathrm{SI}_{\mathrm{S} / \mathrm{N}}=\frac{\min \left(\Sigma S / N_{\text {left }}, \Sigma S / N_{\text {right }}\right)}{\max \left(\Sigma S / N_{\text {left }}, \Sigma S / N_{\text {right }}\right)}
$$

where $\Sigma S / N_{\text {left }}$ is the sum of the $\mathrm{S} / \mathrm{N}$ of all single-pulse events (within the SPEG) to the left of the brightest single-pulse event, and $\Sigma S / N_{\text {right }}$ is the corresponding sum of all single-pulse events (within the SPEG) to the right. Note that both $\mathrm{SI}_{\mathrm{DM}}$ and $\mathrm{SI}_{\mathrm{S} / \mathrm{N}}$, defined by equations (4.6) and (4.7) respectively, have a range between 0 and 1. The higher these two indices are, the more symmetric the SPEG would be. In contrast, RFI SPEGs that do not follow the law described in Equation (4.1) typically appear to be monotonically decreasing (or increasing) in the $\mathrm{S} / \mathrm{N}$ versus DM space. Therefore, they usually have symmetry indices close to 0 and can be differentiated from astrophysical SPEGs which are usually more symmetric;

3. For a non-clipped SPEG, $\mathrm{SI}_{\mathrm{DM}}>0.20$ and $\mathrm{SI}_{\mathrm{S} / \mathrm{N}}>0.25$ ensure that the brightest single-pulse event of the SPEG is in the middle and the $\mathrm{S} / \mathrm{N}$ versus DM curve is balanced to a certain degree. On the other hand, for a clipped SPEG, only $\mathrm{SI}_{\mathrm{S} / \mathrm{N}}>0.10$ is required because clipping often results in less balanced $\mathrm{S} / \mathrm{N}$ versus DM curve. SPEGs (whether clipped or not) that have even lower symmetry indices are considered as RFI and are excluded from further investigation. In practice, these threshold values are so small that only extremely asymmetric SPEGs (that are unlikely to be astrophysical pulses) were eliminated. In other words, these threshold values are applicable to surveys with different observational setups;

4. Inspired by the roughly Gaussian shape of the $\mathrm{S} / \mathrm{N}$ versus DM curve, we divided a non-clipped SPEG into six sections, with three sections on each side of the 
brightest single-pulse event. In the case of a clipped SPEG, as it is missing the peak, each side was divided into two sections instead. The same rule also applies to non-clipped SPEGs with less than three single-pulse events on either side;

5. Within each section $i$, we applied weighted least square (WLS) linear regression between $\mathrm{S} / \mathrm{N}$ and DM and recorded the slope of the regression line as FLS (Fit Line Slope). The $\mathrm{S} / \mathrm{N}$ of each single-pulse event was used as its weight. We chose WLS linear regression over ordinary least squares (OLS) linear regression which was used in RAPID [26] because the former is more immune to some low $\mathrm{S} / \mathrm{N}$ noise events that are occasionally included into SPEGs. The comparison between OLS linear regression and WLS linear regression is demonstrated through their application on the clipped pulse shown in Figure 4.9. It can be seen that the fit lines obtained from WLS linear regression more accurately reflect the actual shape of the $\mathrm{S} / \mathrm{N}$ versus DM curve. For a clipped pulse, we also recorded the DM of the intersection of the two middle fit lines as the center DM (centerDM), the hypothetical DM of the clipped peak;

6. For each $\mathrm{SPEG}$, we define a slope threshold $\mathrm{SL}_{\text {th }}$ for its fit lines as follows:

$$
\mathrm{SL}_{\mathrm{th}}=\max \left(0.01 / \mathrm{DM}_{\text {spacing }}, 0.01 \mathrm{pc}^{-1} \mathrm{~cm}^{3}\right)
$$

where $\mathrm{DM}_{\text {spacing }}$ is the $\mathrm{DM}$ spacing where the $\mathrm{S} / \mathrm{N}$ peaks and it is defined as:

$$
\mathrm{DM}_{\text {spacing }}=\mathrm{DM}_{\mathrm{n}+1}-\mathrm{DM}_{\mathrm{n}}
$$

where $\mathrm{DM}_{\mathrm{n}}$ is the $\mathrm{DM}$ where the $\mathrm{S} / \mathrm{N}$ peaks, and $\mathrm{DM}_{\mathrm{n}+1}$ is $\mathrm{DM}$ of the next trial $\mathrm{DM}$ channel. Therefore $\mathrm{SL}_{\text {th }}$ is DM-spacing-dependent. We made the slope threshold dependent on DM spacing because we believe for a fit line to be considered as steep, its $\mathrm{S} / \mathrm{N}$ should on average display a minimum change of 0.01 for a DM change of one DM spacing. (Note that we further set a lower bound of 0.01 for $\mathrm{SL}_{\text {th }}$ for $\mathrm{DM}_{\text {spacing }}>1 \mathrm{pc}^{-1} \mathrm{~cm}^{3}$, which is equivalent to requiring a minimum $\mathrm{S} / \mathrm{N}$ change of 0.01 for a DM change of $1 \mathrm{pc}^{-1} \mathrm{~cm}^{3}$.) Even smaller change in $\mathrm{S} / \mathrm{N}$ over one DM step indicates a flat $\mathrm{S} / \mathrm{N}$ versus $\mathrm{DM}$ curve, therefore a score of 0 is assigned to the fit line. We selected such a small $\mathrm{SL}_{\mathrm{th}}$ because a larger threshold would cause dim and/or very wide pulses to be overlooked. An example of a wide, not very bright pulse is shown in Figure 4.10. It can be seen that the slopes of its fit lines are fairly small. 

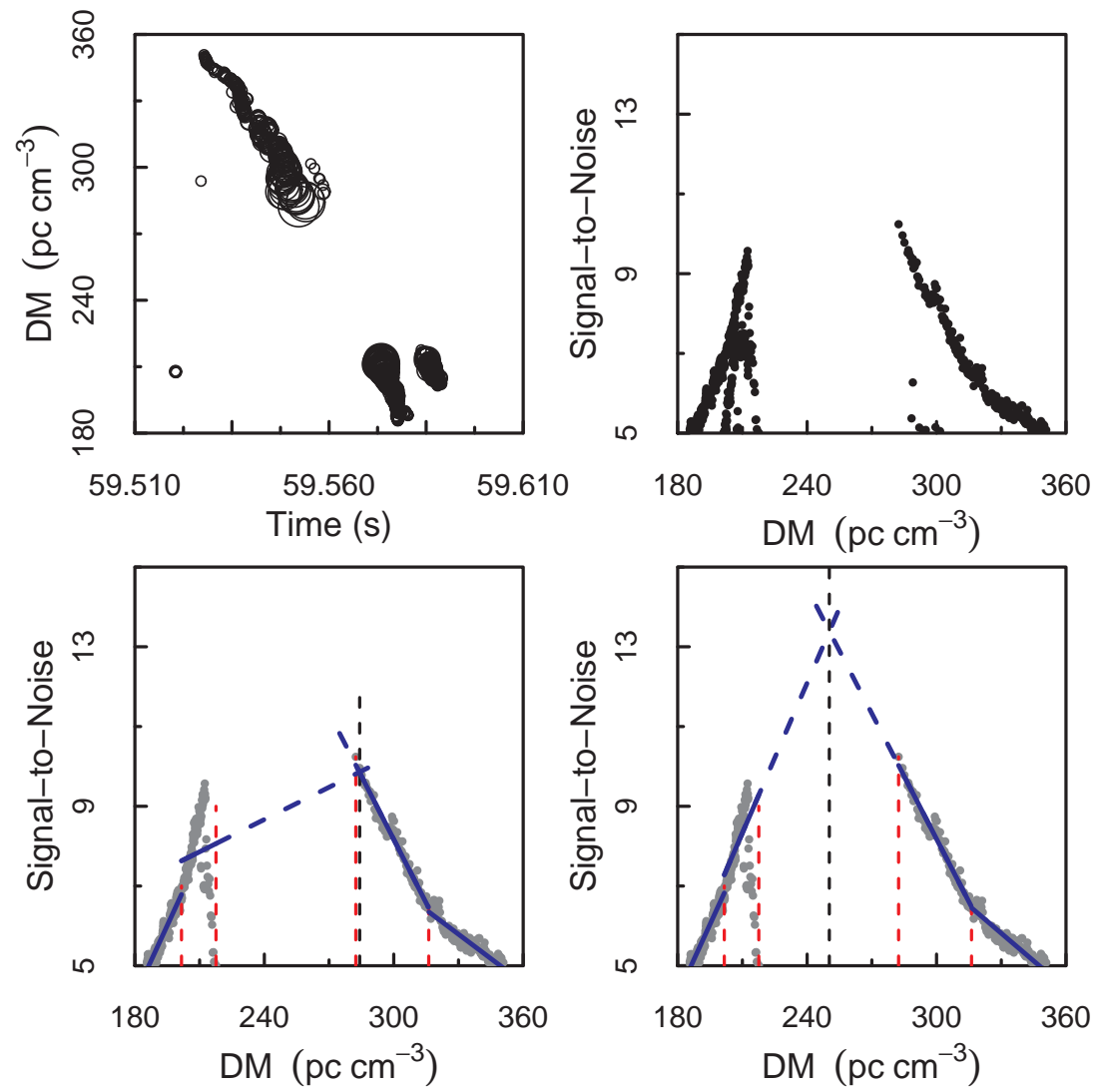

Figure 4.9: The application of OLS linear regression and WLS linear regression on a clipped pulse. The top left subplot shows the SPEG in the DM versus time space, while the other three plots show the $\mathrm{S} / \mathrm{N}$ versus DM curve. While the top right subplot shows all single-pulse events within the SPEG, the bottom two plots only keep the brightest event if there is more than one event at the same DM. In the bottom left subplot, the fit lines were obtained through OLS liner regression, and the second fit line is strongly influenced by those dim events, whereas in the bottom right subplot, their influence is significantly reduced by WLS linear regression. The intersection of the middle two fit lines is recorded as the center DM (centerDM), and its value is shown by the black dashed line. It can be seen that centerDM obtained from WLS linear regression is much closer to the DM of the clipped peak.

7. For each section $i$, we compared the slope of the regression line $\mathrm{FLS}_{\mathrm{i}}$ with $\mathrm{SL}_{\mathrm{th}}$

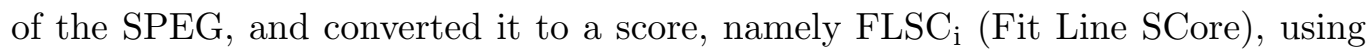



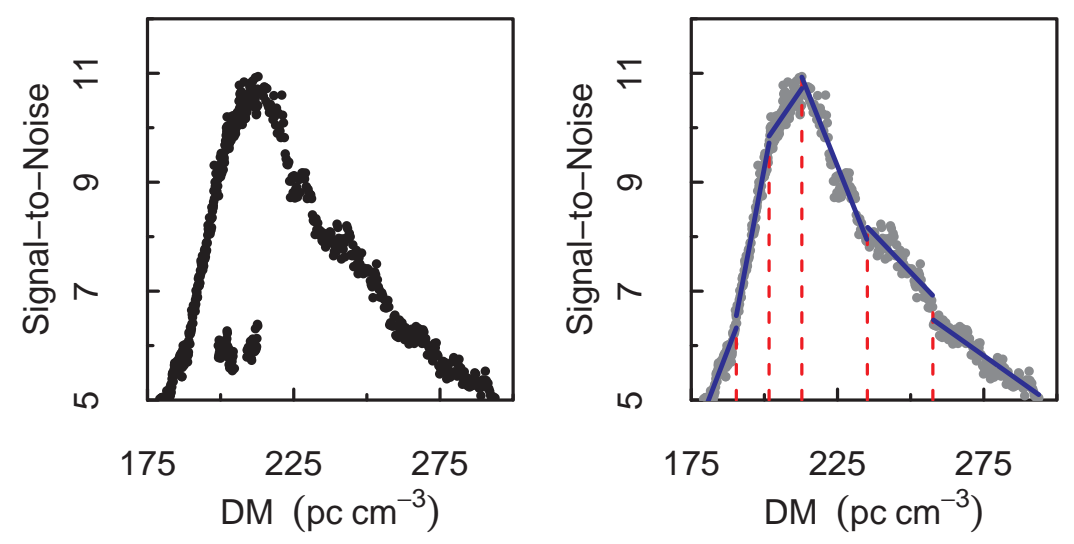

Figure 4.10: An example of a wide, not very bright pulse. Given the DM spacing at the peak $\mathrm{S} / \mathrm{N}$ is $0.3 \mathrm{pc}^{-1} \mathrm{~cm}^{3}, \mathrm{SL}_{\mathrm{th}}$ calculated by equations (4.8) and (4.9) is 0.03 , and the peak score is 6 . The slopes of the six fit lines calculated from WLS linear regression are (from left to right): $0.140,0.283,0.078,-0.134,-0.056$ and $-0.038 \mathrm{pc}^{-1} \mathrm{~cm}^{3}$. Thus using any slope threshold greater than 0.14 will result in a peak score less than 2 , and cause this pulse to be overlooked.

Equation (4.10):

$$
\mathrm{FLSC}_{\mathrm{i}}=\left\{\begin{array}{cl}
-1, & \text { if } \mathrm{FLS}_{\mathrm{i}}<\mathrm{SL}_{\mathrm{th}} \\
1, & \text { if } \mathrm{FLS}_{\mathrm{i}}>\mathrm{SL}_{\mathrm{th}} \\
0, & \text { otherwise }
\end{array}\right.
$$

8. Finally, we calculated the peak score of the SPEG using Equation (4.11):

$$
\text { Peak Score }=\sum_{i=1}^{n / 2} \text { FLSC }_{i}-\sum_{i=n / 2+1}^{n} \text { FLSC }_{i} \text {, }
$$

where $n$ is the total number of sections that the pulse is divided into (i.e., 6 for a regular pulse and 4 for a clipped pulse). The idea behind Equation (4.11) is that because the $\mathrm{S} / \mathrm{N}$ versus DM curve of an astrophysical pulse is roughly Gaussian, ideally its slope should be positive on the left half of the curve and negative on its right half. Therefore, if a peak is found in an SPEG, Equation (4.11) would return a positive score; otherwise, the returned peak score would be 0 or negative.

Typically, a regular bright pulse has a peak score of 6 (as shown in Figure 4.11), and a clipped pulse has a peak score of 4 (as shown in Figure 4.12). In practice, we found that astrophysical pulses usually have a peak score of at least 2 . Therefore, by 

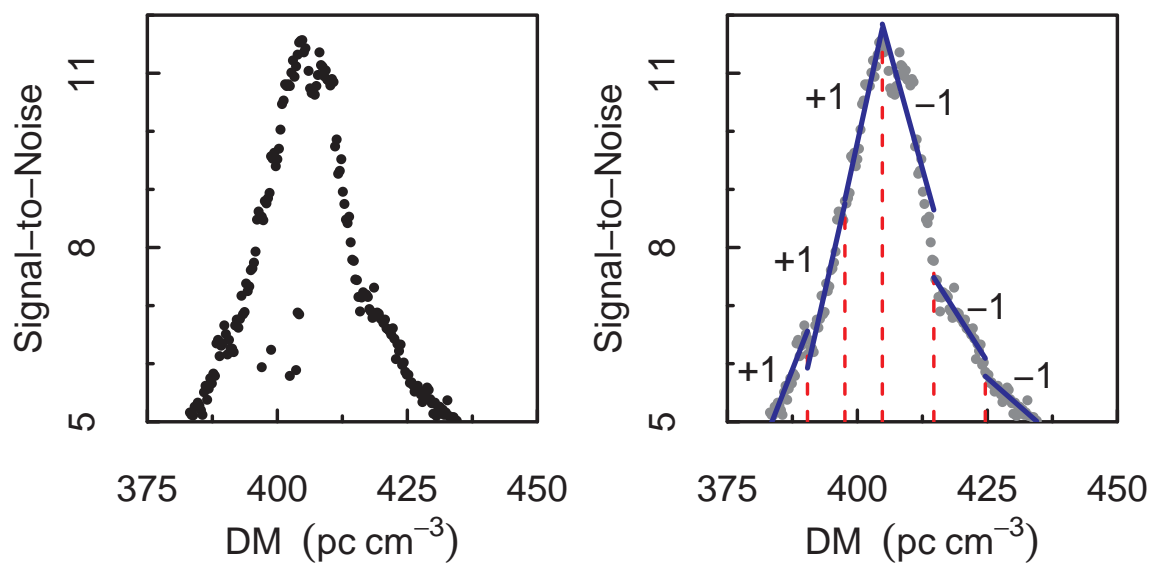

Figure 4.11: Calculating the peak score of a regular pulse from Figure 4.6. While the left subplot shows all single-pulse events within the SPEG, the right subplot shows only the brightest event if there is more than one event at the same DM. Only these events are used in finding the peak score. The calculated peak score for this pulse is 6 .

removing all SPEGs with peak score less than 2, we further denoised an observed beam. An example of such denoising effect is shown in Figure 4.13.
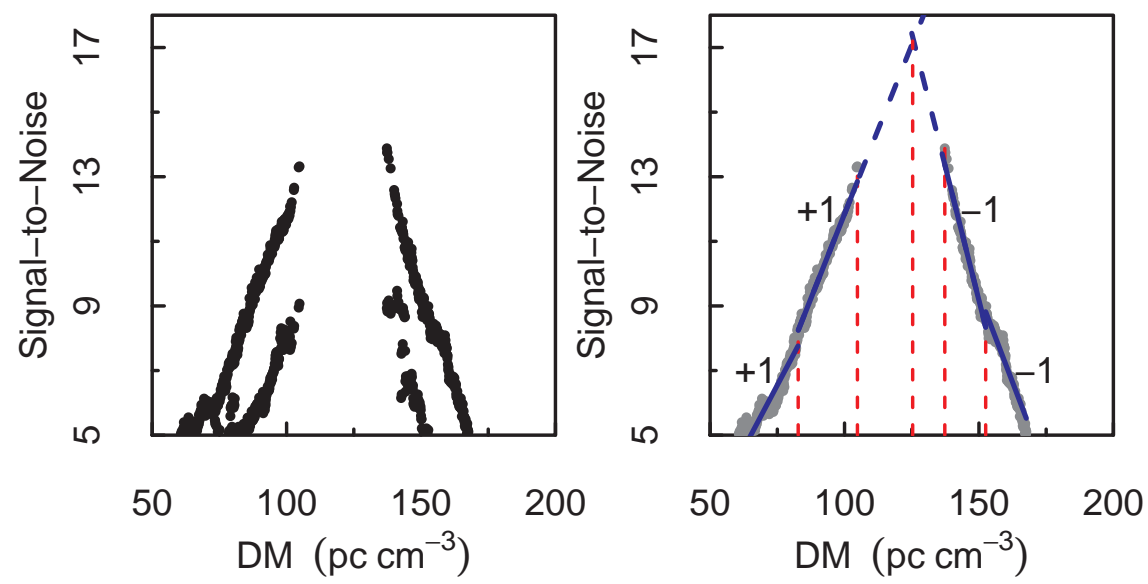

Figure 4.12: Calculating the peak score of a clipped pulse from Figure 4.7. While the left subplot shows all events within the SPEG, the right subplot shows only the brightest event if there is more than one event at the same DM. Only these events are used in finding the peak score. The calculated peak score for this pulse is 4 .

Compared with RAPID [26], we made the following improvements in the peak scoring algorithm of SPEGID:

1. RAPID identifies peaks in the $\mathrm{S} / \mathrm{N}$ versus DM subplot as DPGs, while in SPEGID 

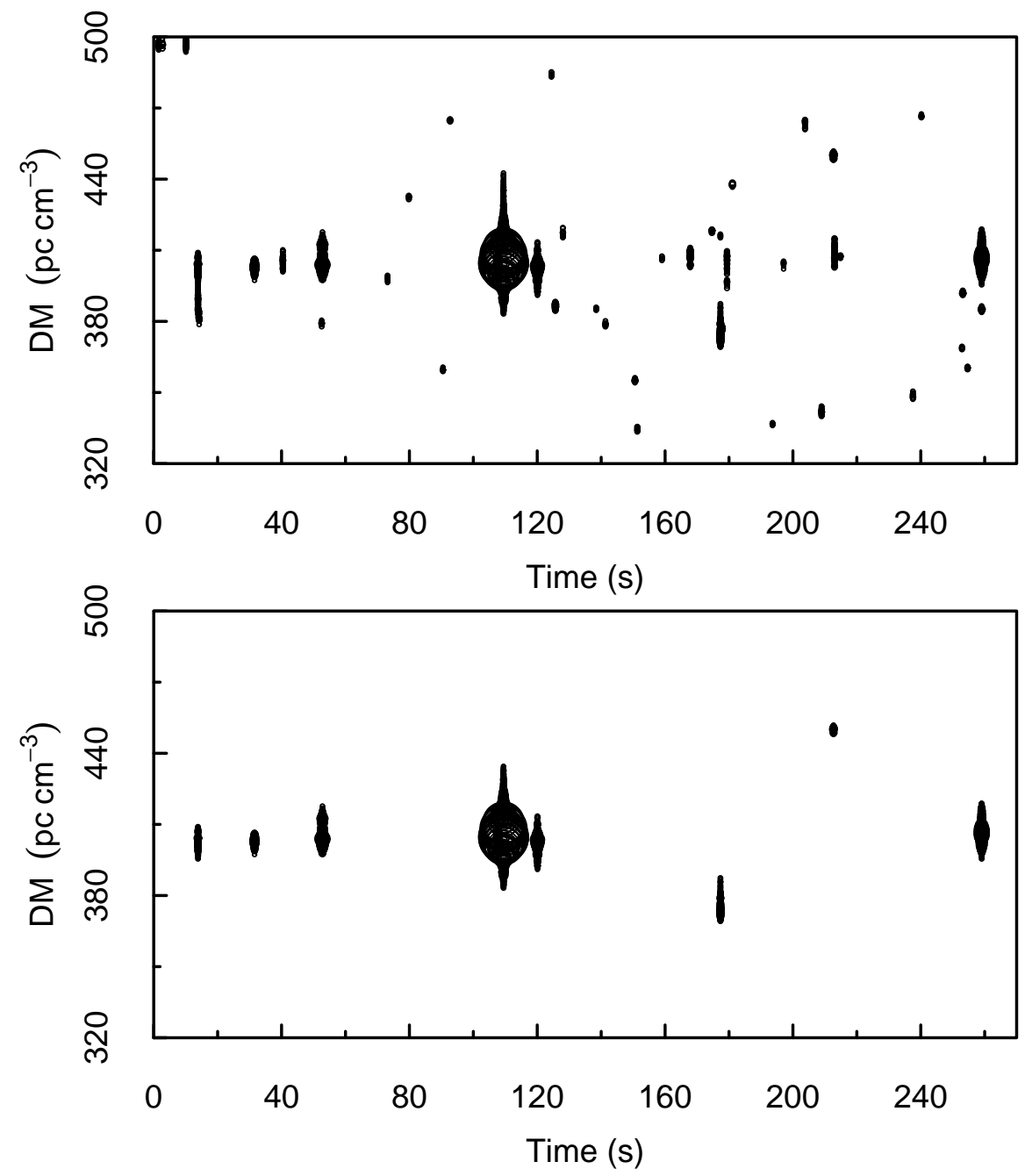

Figure 4.13: The DM versus time plot for pulsar J1901+02. The top subplot shows all SPEGs with a peak score $\geq 2$. By filtering SPEGs with a peak score $<2$, the number of SPEGs is decreased from 81 (shown in the bottom subplot of Figure 4.8) to 48. The bottom subplot only shows those bright SPEGs with a peak score $\geq 2$ and with a peak $\mathrm{S} / \mathrm{N} \geq 6$ (see Section 4.3.1 for the definition of bright SPEGs). These SPEGs are used to form new SPEG groups in Section 4.3.4.

the identification of SPEGs is conducted in the DM versus time space (see Sections 4.3.1 and 4.3.2) and, therefore, separated from calculation of the peak score.

2. In SPEGID, we used a lower, DM-spacing-dependent slope threshold. This allowed us to detect wide (Figure 4.14(a)) and/or dim (Figure 4.14(c, e, g)) pulses despite variable DM spacing of trial DMs was used in different DM ranges.

3. In SPEGID, the number of single-pulse events within each section $i$ is not fixed, 

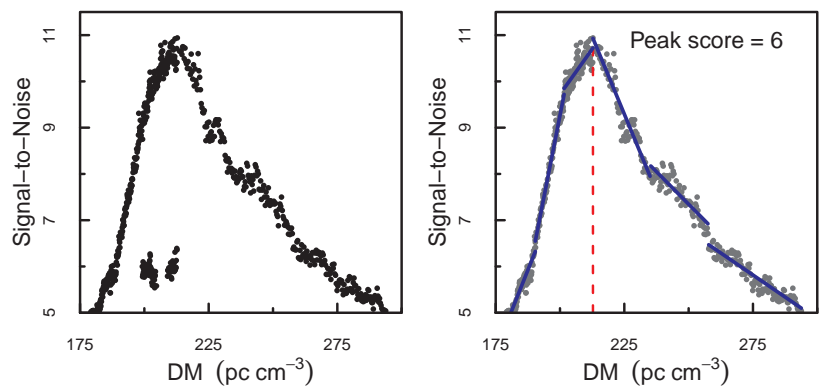

(a)
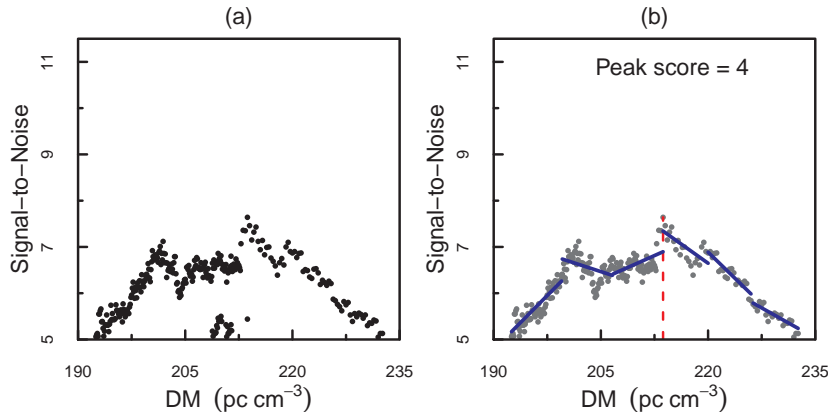

(c)
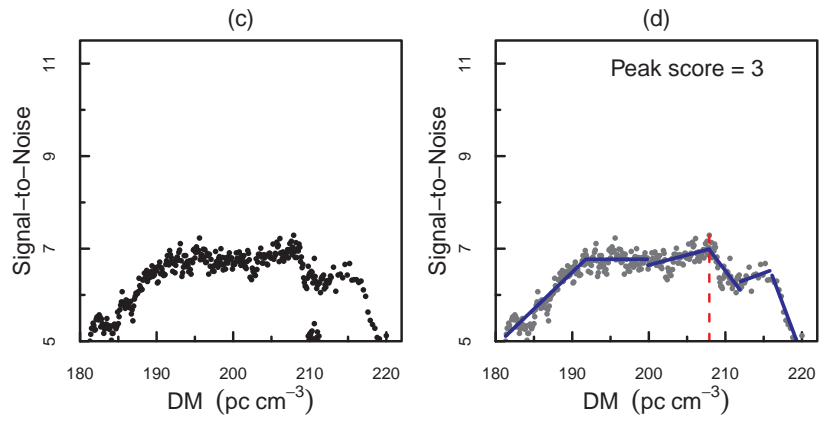

(e)

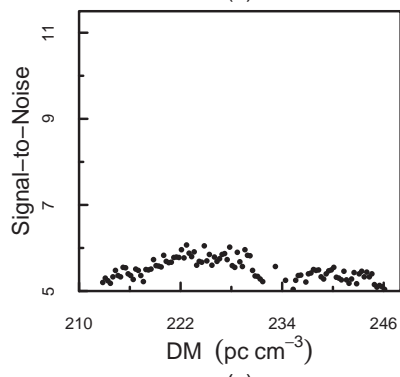

(f)

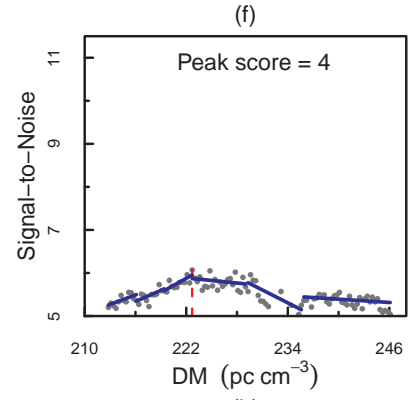

(g)

(h)

Figure 4.14: Examples of astrophysical pulses with different $\mathrm{S} / \mathrm{N}$ versus DM curve shapes (a, c, e, g), and their calculated peak scores (b, d, f, h). These pulses are from the detection of the known pulsar J2003+29 (shown in Figure 4.15) in the PALFA survey. The authenticity of these pulses is confirmed by searching for the underlying periodicity among them and comparing it with the known period of the pulsar (see Section 4.7 for details). The S/N versus DM curve in Figure 4.14(g) looks different than expected, presumably due to the weakness of the pulse. 


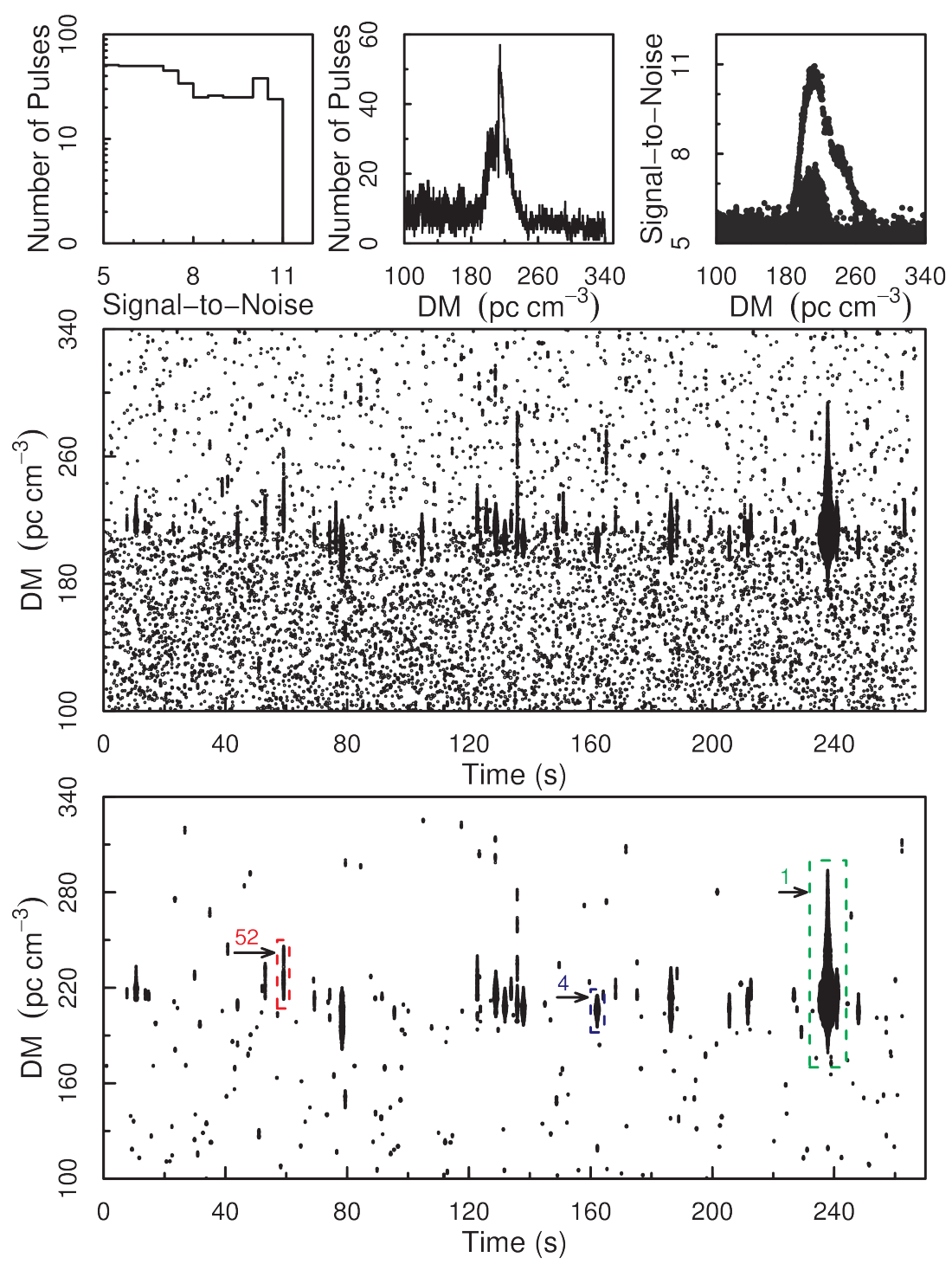

Figure 4.15: Known pulsar J2003+29 detected on MJD 57039 in the PALFA survey. The three subplots in the top include a histogram of the number of single-pulse events versus $\mathrm{S} / \mathrm{N}$, a histogram of the number of single-pulse events versus DM, a scatter plot of $\mathrm{S} / \mathrm{N}$ versus DM. The middle subplot is a scatter plot of the DM versus time for each single-pulse event where its sise is proportional to its $\mathrm{S} / \mathrm{N}$. The bottom subplot shows only SPEGs with peaks identified. The numbers 1, 4 and 52 are the ranks of the SPEGs by their peak $\mathrm{S} / \mathrm{N}$ in descending order.

which is essential for identifying peaks in SPEGs that consist of only a few singlepulse events.

4. Compared with OLS linear regression which was used in RAPID, using WLS linear 
regression makes SPEGID more immune to possible low $\mathrm{S} / \mathrm{N}$ noise events that are occasionally included in SPEGs.

5. A pulse does not always appear as a single Gaussian peak in the DM versus time space, as those shown in Figure 4.14(c, g). RAPID may split such a pulse into more than one DPG. In contrast, SPEGID treats this pulse as a whole SPEG (through the merging step, described in Section 4.3.2) without breaking it, thus can identify the peak-like shape in the $\mathrm{S} / \mathrm{N}$ versus $\mathrm{DM}$ curve of the pulse.

6. While RAPID only returns whether a peak was found or not, the peak score adds additional information which is used as a feature in classification stage (see Section 4.5).

\subsubsection{Grouping SPEGs Horizontally}

If multiple pulses are detected from the same sky position, a consistent DM will increase the chance that they are astrophysical. Therefore, we developed a method to group SPEGs appearing at a consistent DM. This association between pulses was not considered in any known single-pulse search approach [50, 26].

The horizontal grouping of SPEGs is conducted as follows:

1. To account for the fact that the measured DM might vary slightly from pulse to pulse, we define the "central part" of an SPEG as the subset of all single-pulse events falling between the highest and the lowest DM events that have an S/N greater than $90 \%$ of the peak $\mathrm{S} / \mathrm{N}$. The DM range of this central part is then used to group SPEGs.

2. Starting from the brightest SPEG, we compare the DM range of its central part with that of the rest dimmer SPEGs. If the central DM range of the brightest SPEG overlap with that of any dimmer SPEG, and if the DM of the peak S/N of the brightest SPEG fall within the dimmer SPEG's full DM range, then the two SPEGs are considered as appearing at a consistent DM and be placed into the same group. The first condition ensures that the $\mathrm{S} / \mathrm{N}$ versus DM curves of the two SPEGs peak at close DMs. The second condition ensures that the periodicity among SPEGs within the same group can be calculated through single-pulse events detected in the DM channel where the S/N of the brightest SPEG peaks (see Section 4.7).

3. This grouping process is continued until the remaining brightest ungrouped SPEG is no longer considered as a "bright" SPEG (see Section 4.3.1). 
4. Dim SPEGs that are not grouped with any bright SPEG are excluded from further investigation.

Note that the purpose of horizontal grouping is to characterise the association among SPEGs. Just like clustering does not guarantee that every noise event will be excluded from all astrophysical pulses, horizontal grouping alone does not guarantee that all SPEGs from the same source will be placed into the same group, nor that all SPEGs placed in the same group originate from the same source. Nevertheless, horizontal group-
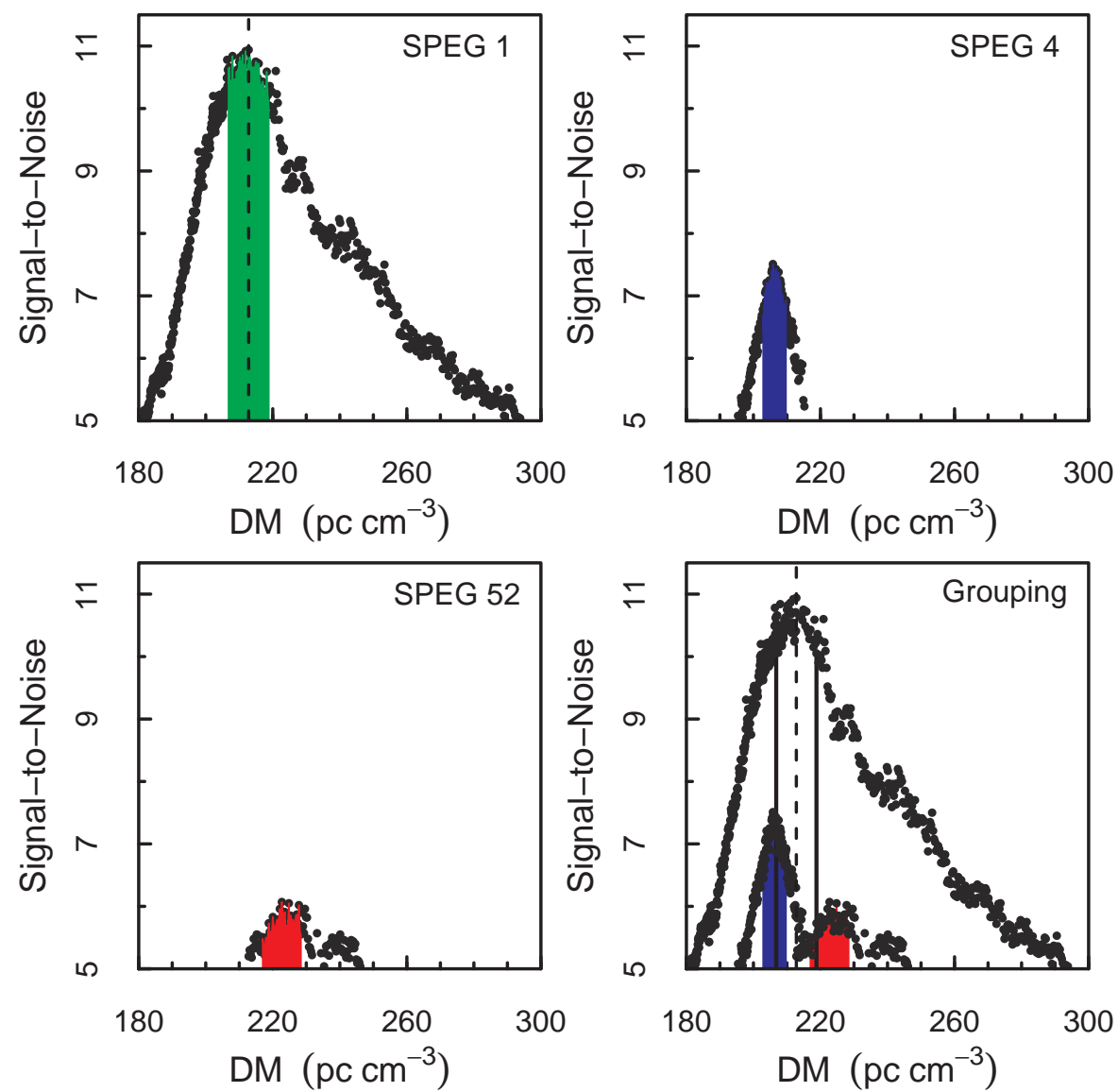

Figure 4.16: S/N versus DM plots of numbered SPEGs 1, 4 and 52 from Fig 4.15. The shaded area in each plot shows the central part of the SPEG. In the bottom right plot, the central part of SPEG 1 is plotted as a transparent region (the two black solid lines represent its DM range) to show its overlap with that of SPEG 3 more clearly. Note that although the DMs of the peak events differ, the central parts of SPEGs 4 and 52 overlap with that of SPEG 1 and the DM of the peak event of SPEG 1 falls into the full DM ranges of SPEGs 4 and 52. Therefore the three SPEGs are grouped together. 
ing of SPEGs provides additional features from SPEG groups. These features, combined with features extracted from individual SPEGs, are likely to improve the automatic classification of SPEGs using supervised machine learning.

Figure 4.15 is a diagnostic plot of the known pulsar J2003+29 detected in the PALFA survey. Compared with the middle subplot where all single-pulse events are shown, the bottom subplot demonstrates again the denoising effect by only displaying SPEGs with peaks identified (i.e., with peak score $\geq 2$ ). We show how horizontal grouping works on SPEGs in Figure 4.16 using the three numbered SPEGs from Figure 4.15. The resultant SPEG groups are displayed in Figure 4.17, with each SPEG group plotted in a different colour.

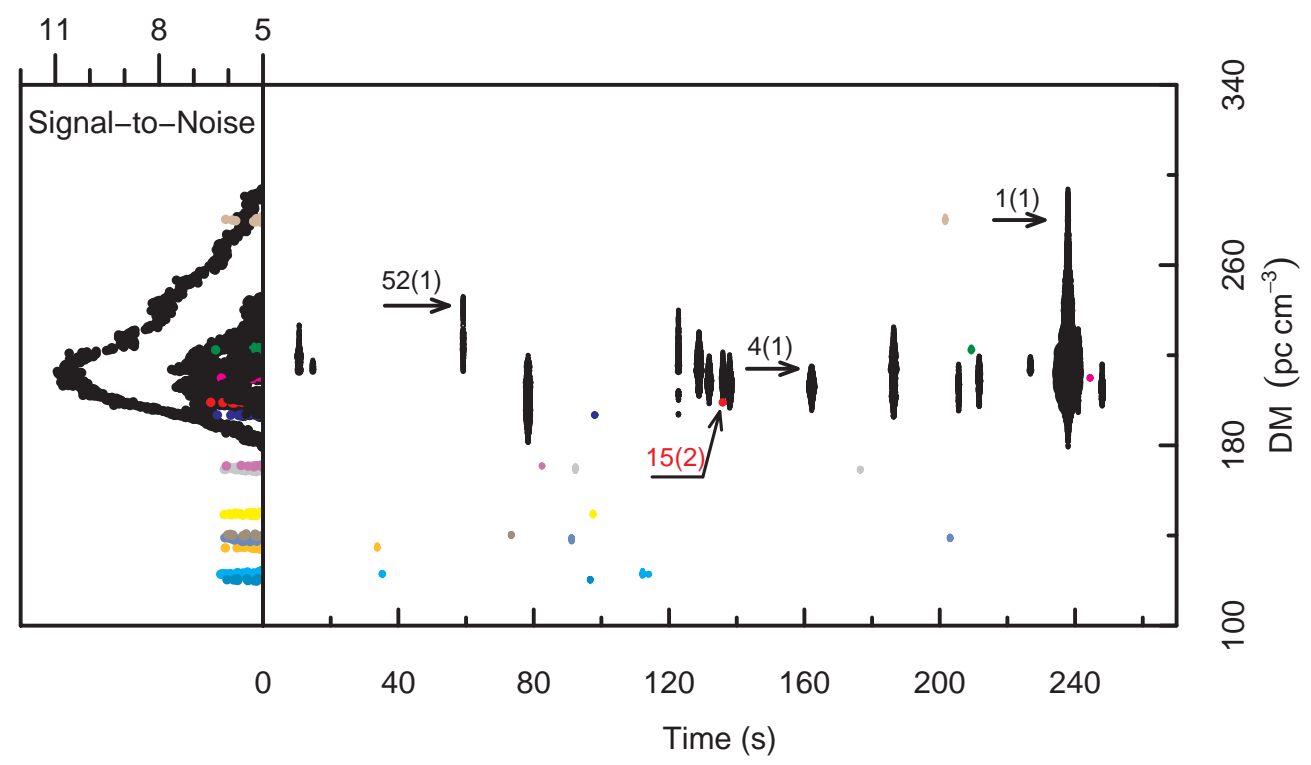

Figure 4.17: Grouped SPEGs from Figure 4.15. Different colours represent different SPEG groups. The number in parentheses is the group number of each SPEG group. For example, SPEG group 1 contains 17 SPEGs (including SPEGs 1, 4, 52, etc.) plotted in black, and SPEG group 2 contains only 1 SPEG (SPEG 15) plotted in red, etc. Each group has at least one bright SPEG, with a peak $\mathrm{S} / \mathrm{N} \geq 6$.

\subsection{Assessment of the Effectiveness of SPEGID}

By applying SPEGID on a beam, we removed isolated noise events and identified pulse candidates as SPEGs through DBSCAN clustering and merging of clusters; we also confirmed the peak-like shape in the $\mathrm{S} / \mathrm{N}$ versus DM curve of SPEGs and filtered out those with peak score less than 2; furthermore, we considered the association between 
SPEGs by horizontally grouping SPEGs appearing at a consistent DM into the same group. These procedures combined together exhibited significant denoising effect in the DM and time space and made astrophysical pulses more noticeable. As a result, the processed diagnostic plots could be examined with much less effort. In fact, we manually inspected these plots for all the beams in the benchmark data set (see Section 4.5.2) and were able to recognise pulsars more easily than in the original diagnostic plots. However, as mentioned earlier, manual inspection can be both tedious and time consuming because of the large number of diagnostic plots. Besides, with ever-increasing data in modern surveys, manually inspecting all diagnostic plots becomes impractical. Hence, automatic classification of the identified SPEGs is necessary, as done in the second stage of our approach.

\subsection{Automatic Classification of SPEGs Using Supervised Machine Learning}

To achieve automatic classification of SPEGs using supervised machine learning, the algorithms are required to acquire the concept of "pulsar" from training examples. This is an example of concept learning [72], which refers to the task of inferring the general definition of a certain concept from training examples that are labeled as members (i.e., pulsars) or nonmembers (i.e., non-pulsars) of the concept. Generally speaking, the purpose of concept learning is to find a target function (or target concept) that can correctly map unseen instances to a Boolean value based on their features (or attributes). Given a set of training examples, learning happens when the learner tries to estimate the target function with a proper hypothesis. Hence concept learning can be viewed as the exercise of searching through the hypothesis space [72] for one that best approximates the target concept.

In supervised machine learning, as stated in the "no free lunch" (NFL) theorem, two supervised machine learning algorithms (i.e., learners) on average exhibit equivalent performance across all possible problems $[110,40]$. Recall that concept learning can be seen as searching for the hypothesis that best approximates the target concept; yet, different hypothesis representations (e.g., linear function, decision tree, rule, artificial neural networks, etc) implicitly define different hypothesis spaces. Therefore, it is necessary to test a wide range of different learners, especially having in mind the lack of research on automatic classification in single-pulse searches. 
In the second stage of our approach, to carry out the automatic classification of SPEGs, we first create a fully labeled benchmark data set, which is used to find the best learner and train a classifier with it thereafter. This trained classifier is then used to automatically classify the SPEGs identified from the unlabeled full data set using SPEGID. Next, we describe the second stage in detail.

\subsubsection{Feature Extraction}

Before supervised learning techniques can be applied, we need to extract features from SPEGs, which are listed in Table 4.1. These features are obtained from three different sources: one feature of the beam, twelve features of individual SPEGs, and five features of the SPEG group. By doing so, our list of features reflect not only the characteristics of individual SPEGs, but also possible association among them. Although SPEG groups (from which five features are extracted) include both bright and dim SPEGs, only bright SPEGs (see Section 4.3.1) are further classified.

Note that $\chi^{2}$ of $\mathrm{S} / \mathrm{N}$ versus DM is usually used to measure how well the shape of the observed pulse in the $\mathrm{S} / \mathrm{N}$ versus $\mathrm{DM}$ space fits the ideal, theoretical shape of a dedispersed pulse given by Equation (4.1). However, $\chi^{2}$ was not included in our feature list due to the following two reasons: (i) The fitting may take a long time and it does not converge for every SPEG, making it impractical for the large number of SPEGs identified in our data set; (ii) Previous study showed that $\chi^{2}$ was not a distinguishing feature (i.e., a good predictor) [26].

\subsubsection{Creating the Benchmark Data Set}

In supervised machine learning, the learners have to be trained on a fully labeled training data set before they can be used to classify new (unseen) data. For that purpose, we carefully created a fully labeled benchmark data set that consists of 90 beams that included detections of 60 distinct pulsars or RRATs identified in the PALFA survey. These pulsars or RRATs are recorded in either the ATNF pulsar Catalog [65] or the PALFA New Pulsars List. ${ }^{3}$ Our selection criterion was to include as many representative diagnostic plots as possible based on their appearances. Because the appearance of a pulsar may vary in different detections, for some pulsars, diagnostic plots of different detections were included. In addition to the 90 beams with known pulsars, our benchmark includes 900 beams with no known pulsars or RRATs found in them. If counted

\footnotetext{
${ }^{3}$ http://www .naic .edu/ palfa/newpulsars/http://www.naic.edu/ palfa/newpulsars/
} 
Table 4.1: Features extracted for each SPEG and used by machine learning algorithms for classification.

\begin{tabular}{|c|c|c|}
\hline & Feature & Description \\
\hline 1 & ClusterDensity & $\begin{array}{l}\text { Density of clusters in the DM versus time space of this particular } \\
\text { beam, defined by Equation (4.3) }\end{array}$ \\
\hline 2 & PeakS/N & Maximum $\mathrm{S} / \mathrm{N}$ of the SPEG \\
\hline 3 & PulseWidth & $\begin{array}{l}\text { Width of the brightest single-pulse event within the SPEG, obtained } \\
\text { from matched filtering }\end{array}$ \\
\hline 4 & CentreDM & $\begin{array}{l}\text { For a regular SPEG, the DM of the peak S/N; for a clipped SPEG, } \\
\text { the determined central DM, as described in Section } 4.3 .3\end{array}$ \\
\hline 5 & DMWidth & $\begin{array}{l}\text { SPEG's width in DM (maximum DM of the SPEG minus minimum } \\
\text { DM) }\end{array}$ \\
\hline 6 & TimeWidth & $\begin{array}{l}\text { SPEG's width in time (maximum time of the SPEG minus minimum } \\
\text { time) }\end{array}$ \\
\hline 7 & Clipped & $\begin{array}{l}\text { center value representing whether the SPEG is clipped, described in } \\
\text { Section } 4.3 .3\end{array}$ \\
\hline 8 & $S I_{\mathrm{DM}}$ & $\begin{array}{l}\text { Numerical value measuring the symmetry of the SPEG by DM, de- } \\
\text { fined by Equation }(4.6)\end{array}$ \\
\hline 9 & $S I_{\mathrm{S} / \mathrm{N}}$ & $\begin{array}{l}\text { Numerical value measuring the symmetry of the SPEG by S/N, de- } \\
\text { fined by Equation (4.7) }\end{array}$ \\
\hline 10 & PeakScore & Peak score of the SPEG, defined by Equation (4.11) \\
\hline 11 & SPEGRank & Rank of the SPEG by PeakS/N within the beam \\
\hline 12 & WidthRatio & Ratio of TimeWidth over PulseWidth \\
\hline 13 & SizeRatio & $\begin{array}{l}\text { Ratio of number of single-pulse events in the SPEG over number of } \\
\text { DM channels in which single-pulse events are detected }\end{array}$ \\
\hline 14 & GroupMaxS/N & Peak S/N of brightest SPEG in the group \\
\hline 15 & GroupRank & Rank of SPEG group by GroupMaxS/N within the beam \\
\hline 16 & GroupMedian $S / N$ & Median of the peak S/N of all SPEGs in the group \\
\hline 17 & BrightRecurTimes & Number of bright SPEGs in the group \\
\hline 18 & RecurTimes & Total number of SPEGs in the group \\
\hline
\end{tabular}


by SPEG, our benchmark data set includes 6,386 positively labeled SPEGs and 127,812 non-pulsar SPEGs, all of which were manually labeled.

\subsubsection{Tested Machine Learning Algorithms}

With a goal to select the best performing learner, we test six learners (used in previous study [26]) on the benchmark data set: JRip, J48, PART, RandomForest, SMO (Sequential Minimal Optimization) and MLP (Multilayer perceptron), as listed in Table 4.2. All six learners are implemented in Waikato Environment for Knowledge Analysis (WEKA), which is a popular suite of machine learning software [35]. The six learners represent knowledge in different forms and exhibit different degrees of interpretability [109]. Descriptions of these algorithms can be found in Chapter. 2.

Table 4.2: List of machine learning algorithms used for the classification of SPEGs.

\begin{tabular}{cc}
\hline Learner & Type \\
\hline JRip [15] & Rule \\
J48 [87] & Tree \\
PART [36] & Rule + Tree \\
RandomForest [9] & Ensemble Tree \\
SMO [86] & Support Vector Machine \\
MLP [74] & Artificial Neural Network \\
\hline
\end{tabular}

We use five-fold cross-validation to evaluate these learners on the benchmark data set. Specifically, the benchmark data set is first divided into five folds using stratified sampling (i.e. each fold contained an equal number of 18 beams with pulsars detected and 180 beams with no pulsar detected). Every time, different four folds are used to train the learner to build a classifier and the fifth fold is used to test the performance of the classifier. The aggregated results of the five folds (reported in Sections 4.8.1 and 4.8.2) are then used to select the best learner, based on the F-measure (also known as $\mathrm{F}_{1}$ Score ).

\subsubsection{Imbalance Consideration}

In our benchmark data set, only 90 out of 990 beams contain astrophysical pulses. Furthermore, these 90 beams contain 6,386 positively labeled SPEGs, which account for even smaller proportion of the 134,198 SPEGs identified in the whole benchmark data set. With a strongly imbalanced distribution of classes like this, many learners can perform poorly regarding the classification of new observations, especially for the minority class 
(i.e., pulsar class), which is exactly the class of interest. Consequently, we used Synthetic Minority Oversampling TEchnique (SMOTE) to generate a balanced benchmark data set [14] because previous study showed that it outperformed other approaches for imbalance treatments [26]. SMOTE increases the size of the minority class by creating synthetic instances for each sample from the minority class, and specifically, by adding small random perturbations toward its closest real instances (SPEGs) in the feature space to avoid overfitting. When we tested the learners based on the benchmark data set, we only applied SMOTE to SPEGs belonging to the pulsar class in the four folds that were used for training, while the fifth (i.e., testing) fold was used as it was.

\subsubsection{Metrics for Evaluation of the Classifications}

To measure and compare the effectiveness of learners, we calculated several performance metrics that are derived from the confusion matrix shown in Figure 4.18 [109].

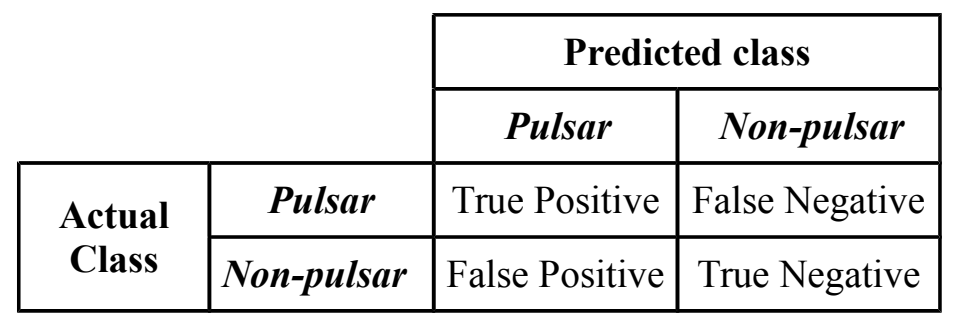

Figure 4.18: Confusion matrix for SPEG classification.

The meanings of the four terms in the confusion matrix are as follows: (i) True Positives (TP): the number of SPEGs that are correctly classified as pulsars; (ii) False Positives (FP): the number of SPEGs that are incorrectly classified as pulsars; (iii) True Negatives (TN): the number of SPEGs that are correctly classified as non-pulsars; (iv) False Negatives (FN): the number of SPEGs that are incorrectly classified as non-pulsars. (v) F-measure $\left(F_{1}\right)$ : the harmonic mean of the precision and recall, also referred to as $\mathrm{F}_{1}$ Score.

$$
\text { F-measure }=2 \times \frac{\text { Precision } \times \text { Recall }}{\text { Precision }+ \text { Recall }}
$$

Because F-measure is high only if both the precision and recall are high, we used Fmeasure to select the best learner. 


\subsubsection{Automatic Classification of the Unlabeled Full Data Set}

Once the best learner is identified using the benchmark data set, it can be used to classify unlabeled instances. The automatic classification of unlabeled full data set is summarised as follows: (i) We train the best learner on the whole benchmark data set to produce the best classifier (i.e., model); (ii) We apply SPEGID on the full data set to identify SPEGs and extract features from them; (iii) We use the best classifier to automatically classify these SPEGs; (iv) The diagnostic plots of beams containing one or more positively classified SPEGs (by the classifier) are manually inspected to confirm whether these SPEGs candidates are astrophysical.

\subsection{Checking Frequency-time Signatures for Bright SPEGs}

The SPEG candidates automatically classified as belonging to pulsar class (i.e., TP and FP) must be examined manually. When multiple bright SPEGs are detected at a consistent DM, their source can be easily confirmed. However, it is not the case for sources with a few dim SPEG candidates being detected. In practice, we found that if the brightest SPEG candidate has a peak $\mathrm{S} / \mathrm{N} \geq 8$, its astrophysical origin can be verified through inspecting its frequency-time structure. For example, in Figure 4.19 the brightest SPEG at $9.26 \mathrm{~s}$ has a peak $\mathrm{S} / \mathrm{N}$ of 8.36. By examining its frequency-time signature shown in Figure 4.20, we confirmed that this pulse was astrophysical, and its attributes agreed with those of the known pulsar J1910+0728.

\subsection{Searching for Periodicity among Dim SPEGs}

As mentioned in Section 4.3.4, previous single-pulse search approaches examined each pulse candidate individually without taking into account the association among the candidates $[50,26]$. For dim SPEGs with $\mathrm{S} / \mathrm{N}<8$, it is difficult to verify whether they are astrophysical pulses or not through the examination of their frequency-time signature. For this reason, we developed an approach to search for an underlying periodicity among a group of SPEGs by looking for a common divisor among the gaps of arrival times. Given a group of SPEGs, we search for an underlying periodicity among them in the following way:

1. The underlying periodicity is searched among single-pulse events that belong to any of the SPEGs; 


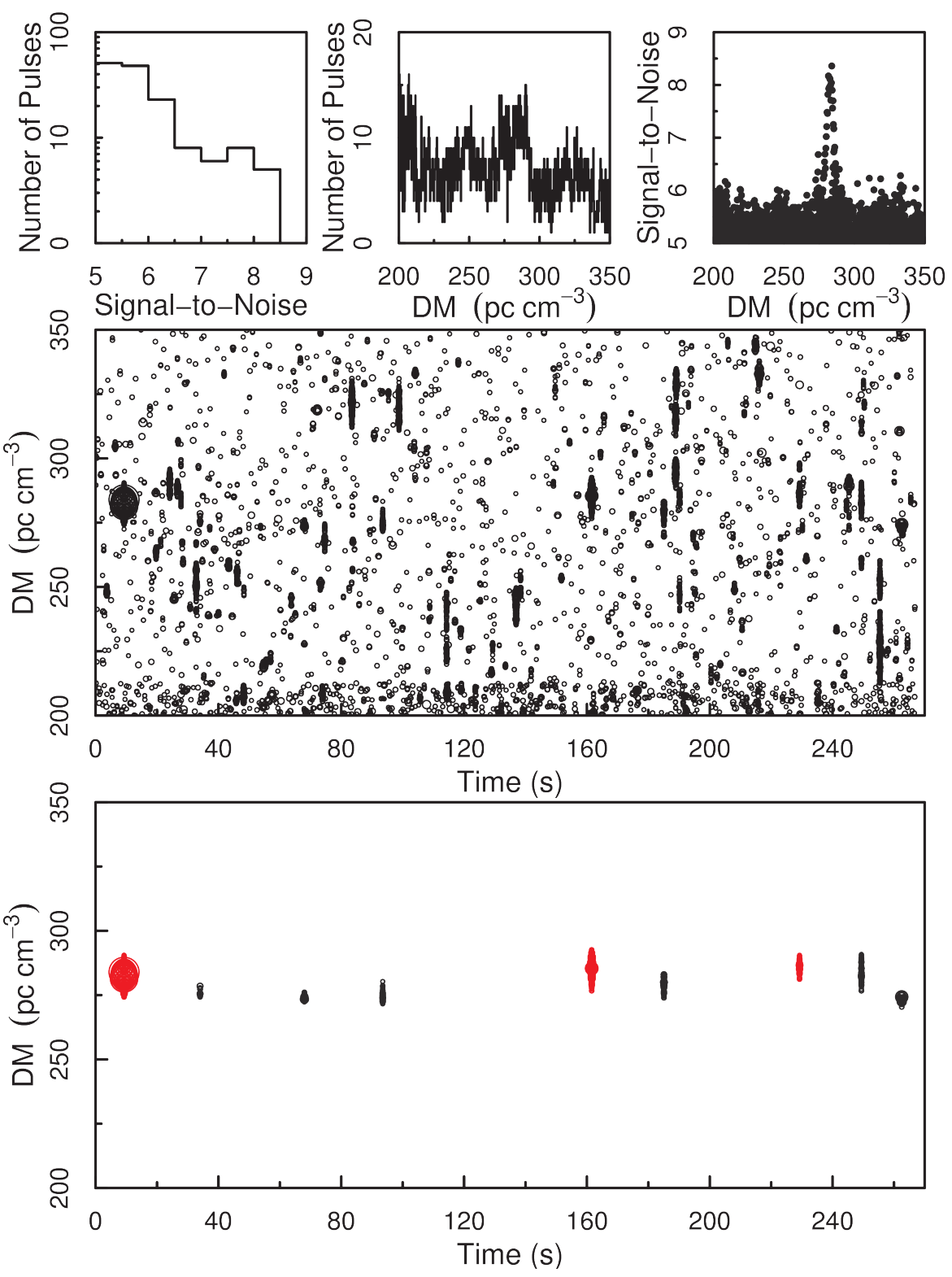

Figure 4.19: Known pulsar J1910+0728 detected on MJD 56663 in the PALFA survey. The bottom subplot shows only SPEGs that have peak score $\geq 2$ and belong to an SPEG group containing at least one SPEG with peak $\mathrm{S} / \mathrm{N} \geq 6$. The red pulses are astrophysical pulses with an underlying period of $0.325 \mathrm{~s}$, which agrees with the period of this pulsar.

2. The underlying periodicity is searched in the DM channel in which the $\mathrm{S} / \mathrm{N}$ of the brightest SPEG (within the group) peaks;

3. The underlying periodicity is searched only when there are $n \geq 3$ single-pulse events that satisfy the previous two constraints; 


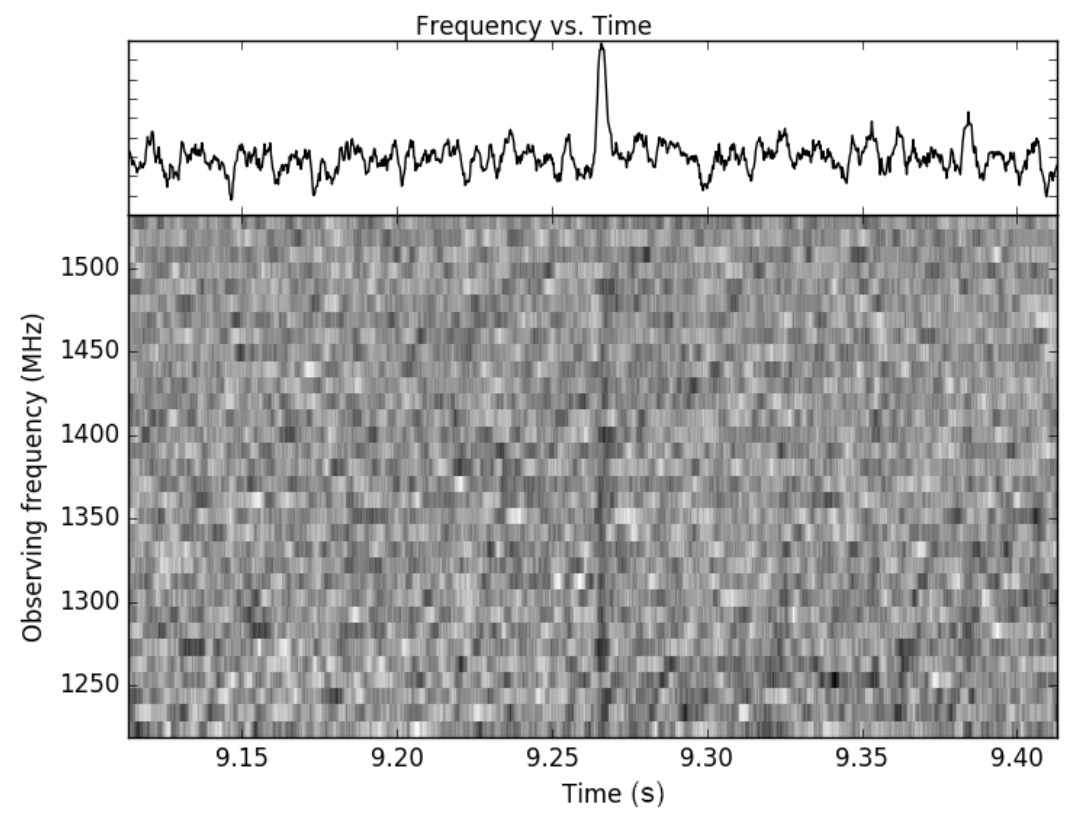

Figure 4.20: The pulse profile (top) and dynamic spectrum (bottom) of the pulse detected at $9.26 \mathrm{~s}$ in Figure 4.19 with maximum $\mathrm{S} / \mathrm{N}=8.36$, dedispersed to $\mathrm{DM}=283.9 \mathrm{pc} \mathrm{cm}^{-3}$ and summed to 32 frequency channels across the band. The total time plotted is 300 ms.

4. Suppose these $n$ single-pulse events are detected at $t_{1}, t_{2}, \ldots, t_{\mathrm{n}}$ respectively, first, we find the time difference $\Delta t_{\mathrm{i}, \mathrm{i}+1}$ between two neighboring single-pulse events as follows:

$$
\Delta t_{\mathrm{i}, \mathrm{i}+1}=t_{\mathrm{i}+1}-t_{\mathrm{i}} \quad(1 \leq \mathrm{i}<\mathrm{n}),
$$

then we find the minimum time difference:

$$
\Delta t_{\min }=\min \left(\Delta t_{\mathrm{i}, \mathrm{i}+1}\right)
$$

5. Once $\Delta t_{\min }$ is determined, a series of period candidates $\left(T_{\mathrm{k}}\right)$ can be obtained by equation (4.15):

$$
T_{\mathrm{k}}=\frac{\Delta t_{\min }}{\mathrm{k}} \quad(k=1,2,3, \ldots),
$$

and all period candidates no less than $0.05 \mathrm{~s}$ are searched. Setting a lower bound for the period candidate is necessary. Otherwise an underlying period will eventually be found among any single-pulse event group. Our preliminary experimentation showed that $0.05 \mathrm{~s}$ was small enough to find the underlying periodicity among astrophysical pluses emitted from known pulsars; 
6. To determine whether a period candidate $T_{\mathrm{k}}$ is valid for two neighboring singlepulse events, we introduce the concept of the tolerance level $(\alpha)$ and compare it with $\beta$, the remainder of $\Delta t_{\mathrm{i}, \mathrm{i}+1}$ divided by $T_{k}$ :

$$
\beta=\left(\left(\frac{\Delta t_{\mathrm{i}, \mathrm{i}+1}}{T_{k}}\right) \bmod 1\right) .
$$

If $\beta<\alpha$, or $\beta>1-\alpha$, then this period candidate $T_{\mathrm{k}}$ between these two events is considered valid. We typically set $\alpha$ between 0.01 and 0.05 , and lower $\alpha$ value indicates more precise periodicity;

7. If the period candidate $T_{\mathrm{k}}$ is valid for all neighboring event pairs, then an underlying periodicity (with a period of $T_{\mathrm{k}}$ ) is found among these $\mathrm{n}$ single-pulse events.

8. If none of the period candidates is valid for these $\mathrm{n}$ events, then we search for an underlying periodicity in all subsets with $n-1$ events using the same procedure described from (iii) to (vii);

9. The searching stops either when there are less than three events within the group, or when a valid period is found among all single-pulse events within the group.

As can be seen in Table 4.3 (which shows the probability of finding an underlying periodicity among a group of random single-pulse events), when four or more SPEGs are detected at a consistent DM, the probability of them being random events decreases significantly if a periodicity among them is found.

From the results listed in in Table 4.3, we can make the following conclusions:

1. A periodicity will almost certainly be found among three single-pulse events;

2. When a highly precise period is found among four or more single-pulse events, or a less precise period is found among five or more single-pulse events, these events are more likely to be astrophysical signals than random noise.

An example of a pulsar with only a series of fairly dim pulses being detected is shown in Figure 4.21. The top three and the middle subplots make up the standard diagnostic plot, in which all single-pulse events within the DM range are plotted. It can be seen that it is difficult, if not impossible, to identify the astrophysical pulses (plotted in red in the bottom subplot) when this plot is manually inspected. In contrast, the bottom subplot only shows SPEGs that have peak score $\geq 2$ and belong to SPEG groups containing at least one SPEG with peak $\mathrm{S} / \mathrm{N} \geq 6$, in which the astrophysical pulses can be more easily 
Table 4.3: The probability of finding an underlying periodicity among random singlepulse events. Tolerance level $(\alpha)$ reflects how consistent the time differences (between any two neighboring events) are with the predetermined period. Lower $\alpha$ value indicates higher consistency. Note that depending on the minimum period searched and the length of the data set (we used $268 \mathrm{~s}$ in our experiments), the values in this table may vary slightly. Nonetheless, the trend will be similar.

\begin{tabular}{lr|rrr}
\hline & Number of events & 3 & 4 & 5 \\
Tolerance level $(\alpha)$ & & & \\
\hline 0.01 & 0.955 & 0.113 & 0.00161 \\
\hline 0.05 & 0.992 & 0.833 & 0.183 \\
\hline
\end{tabular}

identified. Our approach identified ten SPEGs in this diagnostic plot at the DM of 181.1 pc $\mathrm{cm}^{-3}$. However, even the brightest SPEG only has a peak $\mathrm{S} / \mathrm{N}$ of 6.58 , which makes checking its frequency-time signature to confirm its astrophysical nature difficult (see Section 4.6). Nonetheless, we found an underlying periodicity among these ten SPEGs with period of $0.080133 \mathrm{~s}$ (at $\alpha=0.02$ ). Because the position of the beam is close to the known pulsar J1935+2025 at the DM of $182 \mathrm{pc} \mathrm{cm}^{-3}$ with a period of $0.080118 \mathrm{~s}$, we conclude that these ten pulses were from the pulsar J1935+2025.

Note that we included recurrence times (instead of whether the SPEG belonged to a periodic SPEG group or not) as a feature for classification (see Table 4.1). This is because the probability of finding periodicity among three or four single-pulse events is high, and when the number of SPEGs within an SPEG group is large, calculating periodicity becomes time consuming. Hence we only searched for periodicity among SPEG groups that contained one or more positively classified SPEGs. If an underlying periodicity was identified, we then visually inspected the SPEGs and recorded the most promising candidates for future follow-up observation. In contrast, SPEG groups that consisted of mostly dim SPEGs with no underlying periodicity found among them, were classified as RFI.

\subsection{Results}

In this section, we first evaluate the performance of the six learners on the benchmark data set, using the metrics described in Section 4.5.5, with the goal of finding the best learner. Then we present the classification results of running the model (which is obtained 


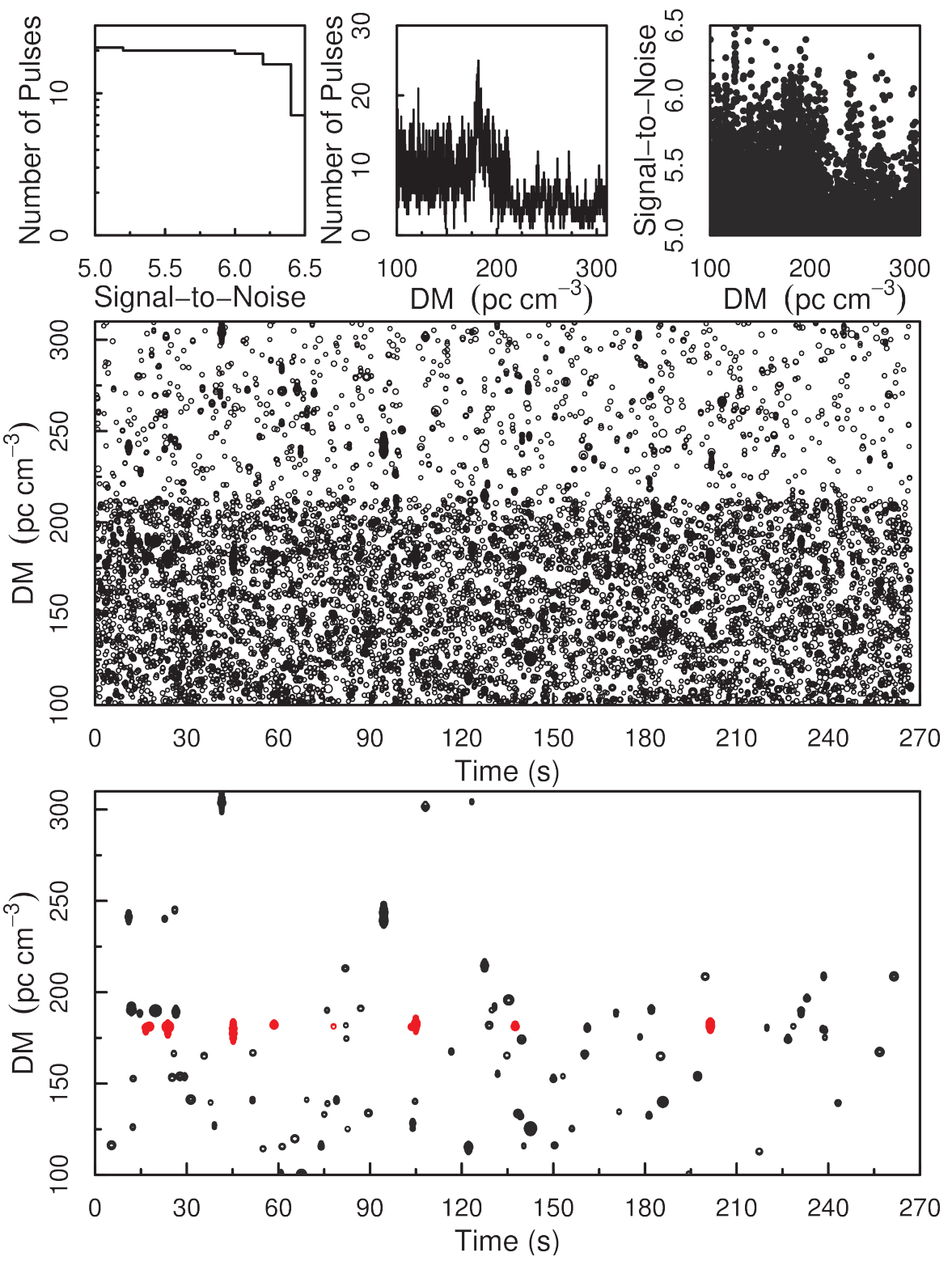

Figure 4.21: Detection of known pulsar J1935+2025 on MJD 56431 in the PALFA survey.

by training the best learner on the whole benchmark data set) on the unlabeled full data set consisting of 47,042 independent beams that are observations of 33,536 unique positions from the PALFA survey.

Note that we measured the learners' performance both by SPEG/pulse and by beam. Typically, the confusion matrix (and the corresponding performance metrics) based on SPEGs is likely to differ from the confusion matrix based on beams. This is because multiple pulses are often detected from the same pulsar, and these pulses would be identified by SPEGID as separate SPEGs. Because we manually inspected the beams 
Table 4.4: The results based on the benchmark data set by SPEG.

\begin{tabular}{llrrrr}
\hline Classifier & Treatment & TP & FN & FP & TN \\
\hline JRIP & none & 5787 & 599 & 216 & 127596 \\
J48 & none & 5855 & 531 & 659 & 127153 \\
PART & none & 6154 & 232 & 221 & 127591 \\
RandomForest & none & 6029 & 357 & 54 & 127758 \\
SMO & none & 3585 & 2801 & 327 & 127485 \\
MLP & none & 5614 & 772 & 140 & 127672 \\
& & & & & \\
JRIP & SMOTE & 6136 & 250 & 425 & 127387 \\
J48 & SMOTE & 6107 & 279 & 561 & 27251 \\
PART & SMOTE & 6142 & 244 & 399 & 127413 \\
RandomForest & SMOTE & 6191 & 195 & 212 & 127600 \\
SMO & SMOTE & 5842 & 544 & 4658 & 123154 \\
MLP & SMOTE & 6057 & 329 & 772 & 127040 \\
\hline
\end{tabular}

that contained one or more positively classified SPEGs (by our trained model), as long as one of these SPEGs was correctly classified, we could find the pulsar in the beam. In other words, the misclassification of one or more SPEGs during the automatic classification process does not necessarily result in missing the pulsar automatically. On the other hand, misclassification of some SPEGs is inevitable in both automatic classification and manual inspection. Therefore, we selected the best learner based on the results by beam rather than by SPEG, as our goal was to find as many pulsars (rather than SPEGs) as possible.

\subsubsection{Results Based on the Benchmark Data Set by SPEG}

Table 4.4 shows the confusion matrix of the six learners tested on the benchmark data set, and Table 4.5 shows the corresponding performance metrics. From these two tables, we can make the following observations:

1. Without imbalance treatment, five of the six learners performed reasonably well. SMO was the worst classifier that missed a large portion of SPEGs in the pulsar class;

2. With SMOTE treatment, the Recall of five of the six learners increased, while that of PART showed little change;

3. When measured by F-measure, RandomForest with SMOTE treatment proved to 
Table 4.5: The performance metrics based on the benchmark data set by SPEG.

\begin{tabular}{lrrrrrr}
\hline Classifier & Treatment & Recall & FNR & Precision & FPR & F-measure \\
\hline JRIP & none & 0.906 & 0.094 & 0.964 & 0.002 & 0.934 \\
J48 & none & 0.917 & 0.083 & 0.899 & 0.005 & 0.908 \\
PART & none & 0.964 & 0.036 & 0.965 & 0.002 & 0.965 \\
RandomForest & none & 0.944 & 0.056 & 0.991 & 0.000 & 0.967 \\
SMO & none & 0.561 & 0.439 & 0.916 & 0.003 & 0.696 \\
MLP & none & 0.879 & 0.121 & 0.976 & 0.001 & 0.925 \\
& & & & & & \\
JRIP & SMOTE & 0.961 & 0.039 & 0.935 & 0.003 & 0.948 \\
J48 & SMOTE & 0.956 & 0.044 & 0.916 & 0.020 & 0.936 \\
PART & SMOTE & 0.962 & 0.038 & 0.939 & 0.003 & 0.950 \\
RandomForest & SMOTE & 0.969 & 0.031 & 0.967 & 0.002 & 0.968 \\
SMO & SMOTE & 0.915 & 0.085 & 0.556 & 0.036 & 0.692 \\
MLP & SMOTE & 0.948 & 0.052 & 0.887 & 0.006 & 0.917 \\
\hline
\end{tabular}

be the best learner overall. This agrees with previous study [26].

\subsubsection{Results Based on the Benchmark Data Set by Beam}

As mentioned before, the confusion matrix (and the corresponding performance metrics) based on beams may differ from that based on SPEGs because multiple pulses are often detected from the same pulsar. In order to investigate potential differences, we show the confusion matrix and the performance metrics of the same experiments based on beams in Tables 4.6 and 4.7, respectively.

Compared with the results by SPEGs, similar trends can be seen here. However, there are several different trends worth mentioning, which are described next.

When measured by beam, the six learners showed comparable Recall (hence the FNR) to the results based on SPEGs. However, the FPR of the six learners based on beams increased significantly. Such increase in FPR indicates that SPEGs that were incorrectly classified as pulsars were distributed among many beams.

With SMOTE treatment, the FNR of all six learners decreased to below $5 \%$, but the FPR increased more significantly. Consequently, the F-measure of all six learners with SMOTE treatment decreased. We still used SMOTE because, in case of pulsar classification, the learner with a lower FNR is preferred (when they have comparable FPR).

Among the six learners with SMOTE treatment, RandomForest showed the high- 
Table 4.6: The results based on the benchmark data set by beam.

\begin{tabular}{llrrrr}
\hline Classifier & Treatment & TP & FN & FP & TN \\
\hline JRIP & none & 83 & 7 & 22 & 878 \\
J48 & none & 82 & 8 & 32 & 868 \\
PART & none & 84 & 6 & 38 & 862 \\
RandomForest & none & 81 & 9 & 3 & 897 \\
SMO & none & 41 & 49 & 27 & 873 \\
MLP & none & 81 & 9 & 29 & 871 \\
& & & & & \\
JRIP & SMOTE & 84 & 6 & 53 & 847 \\
J48 & SMOTE & 86 & 4 & 98 & 802 \\
PART & SMOTE & 88 & 2 & 84 & 816 \\
RandomForest & SMOTE & 86 & 4 & 18 & 882 \\
SMO & SMOTE & 88 & 2 & 687 & 213 \\
MLP & SMOTE & 87 & 3 & 193 & 707 \\
\hline
\end{tabular}

est F-measure. Since all six learners had over $93 \%$ recall when SMOTE treatment was applied, the difference in F-measure mainly originated from the difference in precision. Notably, RandomForest had a 2.2\% lower recall compared to PART and SMO, but a significantly higher precision (i.e., $82.7 \%$ compared to $51.2 \%$ and $11.4 \%$ ). This means that with slightly lower recall (i.e., slightly higher FNR), among the positively classified instances RandomForest produced significantly more true positive than false positive instances. RandomForest showed the highest precision (82.7\%) and the lowest FPR $(2.0 \%)$ most likely because it mitigated the problem of overfitting by building multiple decorrelated learning trees and outputting the average of their classification as the final prediction. In contrast, overfitting is commonly seen in decision tree methods and artificial neural networks as they tend to fit the training data very well [85]. Using multiple trees makes the RandomForest classifier lose the interpretability properties (compared to J48 and PART for example), but this is not a problem since we are more concerned with the predictive power of the classifiers.

If $9.3 \% \mathrm{FPR}$ was considered acceptable, one could argue that PART is the best classifier. As for SMO, the extremely high FPR (76.3\% when measured by beam) is likely due to the fact that some dim RFI and/or noise look very similar to astrophysical pulses. Consequently, not only it is hard to differentiate them through manual inspection, the algorithm could also have difficulties identifying effective support vectors that decide the maximum-margin hyperplane that separates the instances in the feature space. 
Table 4.7: The performance metrics based on the benchmark data set by beam.

\begin{tabular}{lrrrrrr}
\hline Classifier & Treatment & Recall & FNR & Precision & FPR & F-measure \\
\hline JRIP & none & 0.922 & 0.078 & 0.790 & 0.024 & 0.851 \\
J48 & none & 0.911 & 0.089 & 0.719 & 0.036 & 0.804 \\
PART & none & 0.933 & 0.067 & 0.689 & 0.042 & 0.792 \\
RandomForest & none & 0.900 & 0.100 & 0.964 & 0.003 & 0.931 \\
SMO & none & 0.456 & 0.544 & 0.603 & 0.030 & 0.519 \\
MLP & none & 0.900 & 0.100 & 0.736 & 0.032 & 0.810 \\
& & & & & & \\
JRIP & SMOTE & 0.933 & 0.067 & 0.613 & 0.059 & 0.740 \\
J48 & SMOTE & 0.956 & 0.044 & 0.467 & 0.109 & 0.628 \\
PART & SMOTE & 0.978 & 0.022 & 0.512 & 0.093 & 0.672 \\
RandomForest & SMOTE & 0.956 & 0.044 & 0.827 & 0.020 & 0.887 \\
SMO & SMOTE & 0.978 & 0.022 & 0.114 & 0.763 & 0.203 \\
MLP & SMOTE & 0.967 & 0.033 & 0.311 & 0.214 & 0.470 \\
\hline
\end{tabular}

Table 4.8: The results based on the full data set by SPEG.

\begin{tabular}{llrr}
\hline Classifier & Treatment & Total & Positive \\
\hline RandomForest & SMOTE & $7,255,112$ & 24,426 \\
\hline
\end{tabular}

Based on the highest F-measure (i.e., both recall and precision being high) and lowest FPR, we selected RandomForest with SMOTE as the best learner to be applied on the unlabeled full data set. By examining the four pulsars that were misclassified by RandomForest with SMOTE treatment, we found that three of them were dim pulsars with less than five pulses detected, while the fourth pulsar was detected at the lowest DM $\left(8.0 \mathrm{pc} \mathrm{cm}^{-3}\right)$ among all known pulsars in the benchmark. In other words, these pulsars were missed because they are different from the other pulsars included in the benchmark data set.

\subsubsection{Results Based on the Full Data Set}

Before the trained model can be used to classify the unlabeled full data set, we first processed the full data set using SPEGID to identify SPEGs and extract features from them. Then, we used the best model (obtained by training the selected best learner RandomForest with SMOTE treatment - on the whole benchmark data set) to classify the SPEGs identified in the unlabeled full data set. The results are listed in Table 4.8.

From Table 4.8 it can be seen that in the SPEG identification stage, from the 47,042 


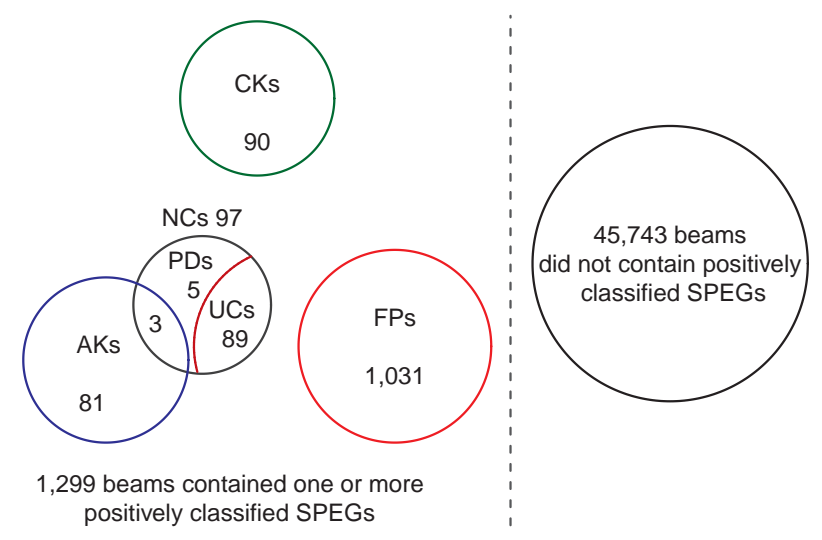

Figure 4.22: A Venn diagram showing the results of automatic classification and manual inspection of beams that contained positively classified SPEG(s). Among these 47,042 beams, only 1,299 beams contained at least one positively classified SPEG. Through quick-look manual inspection of these 1,299 beams, we found all 90 known (60 distinct) pulsars included in the benchmark data set correctly classified (CKs), 84 additional known pulsars not included in the benchmark data set (AKs), 1031 false positives (FPs), i.e., non-pulsars incorrectly classified as pulsars), 89 unlikely candidates (UCs), and 5 possible discoveries (PDs).

beams in the full data set, our proposed approach identified 7,255,112 SPEGs with peak score $\geq 2$. Out of these, 24,426 SPEGs (from 1,299 beams) were classified as pulsars.

Since it was impractical and unnecessary to examine all 24,426 positively classified SPEGs, we manually examined the diagnostic plots of the 1,299 beams from which these 24,426 SPEGs were identified and only present the statistics based on beams here. (Because the same pulsar can be detected in multiple beams, unless we point it out specifically, here we use "pulsar" to refer to the detection of a pulsar within a beam.) Through quick-look manual inspection, the 1299 beams that contained positively classified SPEG candidates were distributed across four categories as follows: 90 known pulsars (60 distinct) included in the benchmark data set were correctly classified (CKs); 81 additional known pulsars not included in the benchmark data set were correctly classified (AKs); 1031 false positives, i.e., non-pulsars incorrectly classified as pulsars (FPs); and 97 new candidates of interest ( $\mathrm{NCs}$ ).

The list of NCs mainly consisted of SPEG groups that were made up of a few fairly dim SPEGs (see Figs. 4.21 and 4.23), which is not surprising as bright pulsars are 
much easier to detect and consequently it is more likely that they have already been discovered. With the aid of techniques described in Sections 4.6 and 4.7, out of the 97 NCs we confirmed 3 additional AKs (making the total number of AKs increase to 84). We also recorded 5 promising candidates as Possible Discoveries (PDs) and classified the rest 89 beams as Unlikely Candidates (UCs). The results are presented in a Venn diagram shown in Figure 4.22.

From these results, the following conclusions can be made:

1. Compared to the total number of 47,042 beams in our data set, our approach reduced the number of beams that required manual inspection to 1,299 (i.e., by over $97 \%$ );

2. Our classifier successfully found all 90 beams containing (60 distinct) known pulsars in the benchmark data set;

3. Our classifier was able to find 84 additional beams containing 49 distinct known pulsars. Among these 49 pulsars, 17 were already included in the benchmark data set. In other words, our classifier found 32 additional distinct known pulsars that were not included in the benchmark date set. Among these 84 beams, 3 beams contained dim detections of known pulsars that could easily be missed by manual inspection. This would be really problematic if these dim detections were the only observations of unknown pulsars. One such example was shown earlier in Fig 4.21;

4. By comparing our discovery list with the PALFA New Pulsars List, we concluded that our classifier was able to detect all known pulsars (that were found by other single-pulse search approaches) in our data set;

5. There were a number of dim periodic candidates that could be pulsars. We included the best five of those as PDs for further exploration. One such example is shown in Figure 4.23. It can be difficult to find these dim candidates through manual inspection. In 2018, we conducted follow-up observation of these promising candidates. Unfortunately, the RFI was very strong and we did not find signals that had the periodicity as candidates in current data. Furthermore, note that SPEGID identified over 4000 five-SPEG groups from those 1120 beams. However, because none of these five-SPEG groups contained any positively classified SPEG (by our trained model), they were classified as UCs and FPs during manual inspection.

6. In total, we found that our single-pulse search approach was able to find 92 out 

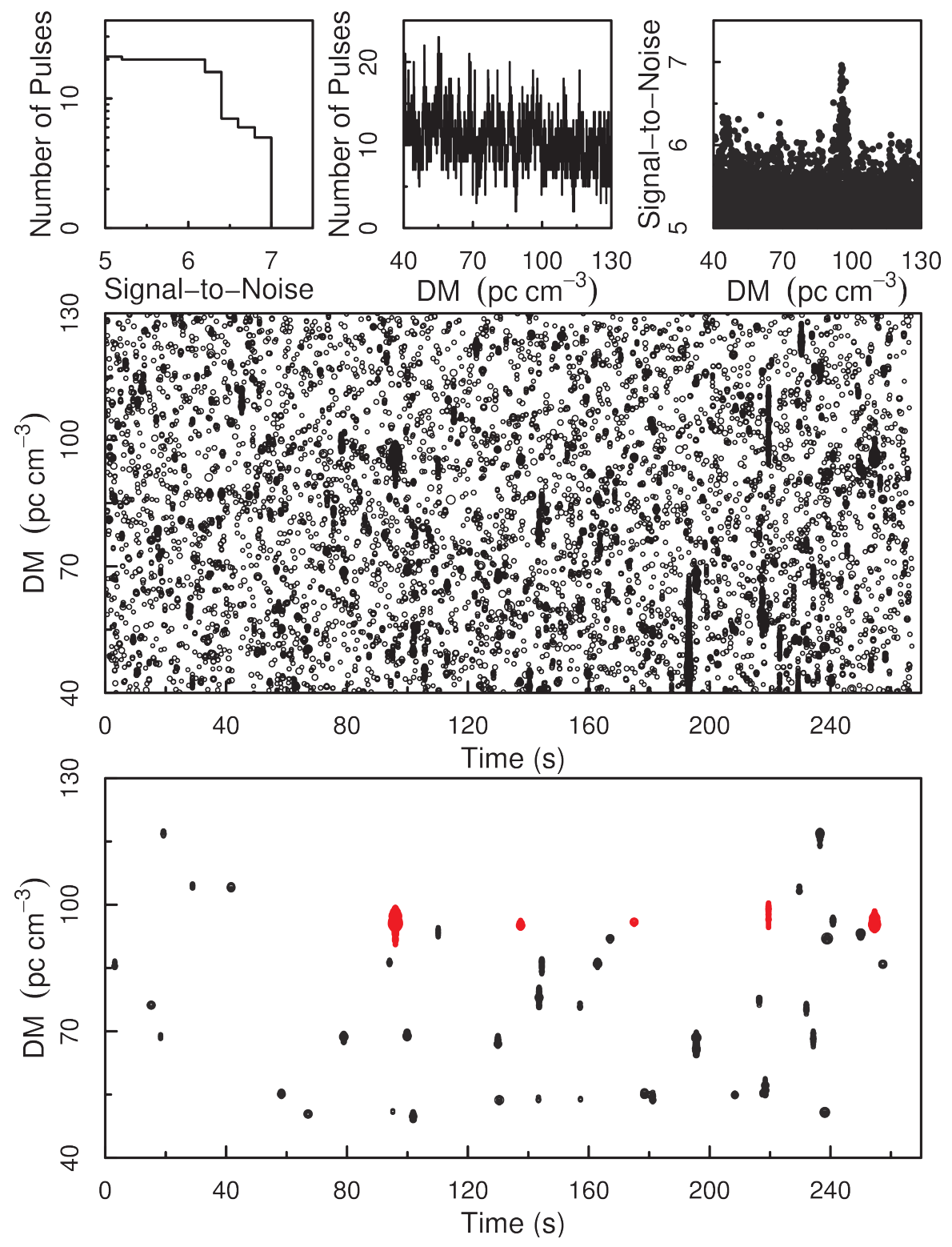

Figure 4.23: One possible discovery. The five SPEGs plotted in red in the bottom subplot are "periodic" at the tolerance level of 0.03 . The probability of finding periodicity among five random events at such tolerance is 0.043 . Nevertheless, it is worth pointing out that the candidate SPEGs are made up of many single-pulse events with a peak-like shape found in their $\mathrm{S} / \mathrm{N}$ versus $\mathrm{DM}$ curve, therefore, the probability of them being purely random should be significantly lower.

of the 284 (i.e., 32.4\%) known pulsars (that were discovered in both single-pulse search and periodicity search approaches) in beams that were positioned within $15^{\prime}$ of their known locations; 
7. During manual inspection, we also found 361 RRAT-like candidates (SPEG groups with one or two good pulse candidates at the same DM with maximum $\mathrm{S} / \mathrm{N}>7$ ) from those 1120 beams that were classified as UCs and FPs. It is known that RRATs usually have few pulses detected within a short observation period. When the RRAT-like candidates are neither bright enough nor can a period be found among SPEGs, it becomes difficult to confirm their astronomical origin through the two approaches described in Sections 4.6 and 4.7. Correspondingly, due to the lack of confirmed examples in the benchmark data set, these RRAT-like candidates were classified as non-pulsars by our classifier.

\subsection{Discovery of a New Pulsar from GBTDrift Survey}

By applying our approach to the GBTDrift survey data, we discovered a pulsar with only two astrophysical pulses observed. Therefore, the underlying periodicity could only be confirmed in the follow-up observation as three or more pulses are needed for calculation of the periodicity.

\subsection{Discussion of the Challenges and Implications}

The automated approach to radio pulsar detection presented in this chapter showed significant improvement compared with previous study [26] if measured by predictive power. More specifically, compared with the best classifier of DPGs, our best classifier of SPEGs exhibited significant increase in F-measure (from $71.6 \%$ to $96.8 \%$ ). (Here Fmeasure by SPEG is used because F-measure by beam was not provided in [26].) We believe this is mainly because we correctly identified individual pulses as separate SPEGs in the DM and time space using DBSCAN clustering and merging the clusters. Therefore, compared with RAPID which identified DPGs in the composite S/N versus DM subplot for an entire observation, we could extract more distinctive features of astrophysical pulses to separate them from RFI and noise.

Applying DBSCAN clustering to identify related single-pulse event groups in the DM versus time space seems natural and fairly simple at first sight because single-pulse events appear to form denser areas. However, in practice, simply applying DBSCAN clustering could end up either grouping unrelated single-pulse events together or splitting related single-pulse events into many clusters because of the following two reasons: (i) Pulses have a wide range of time and DM extents, depending on the pulse width, shape, and 
GBT350drift_54275_0539+0007
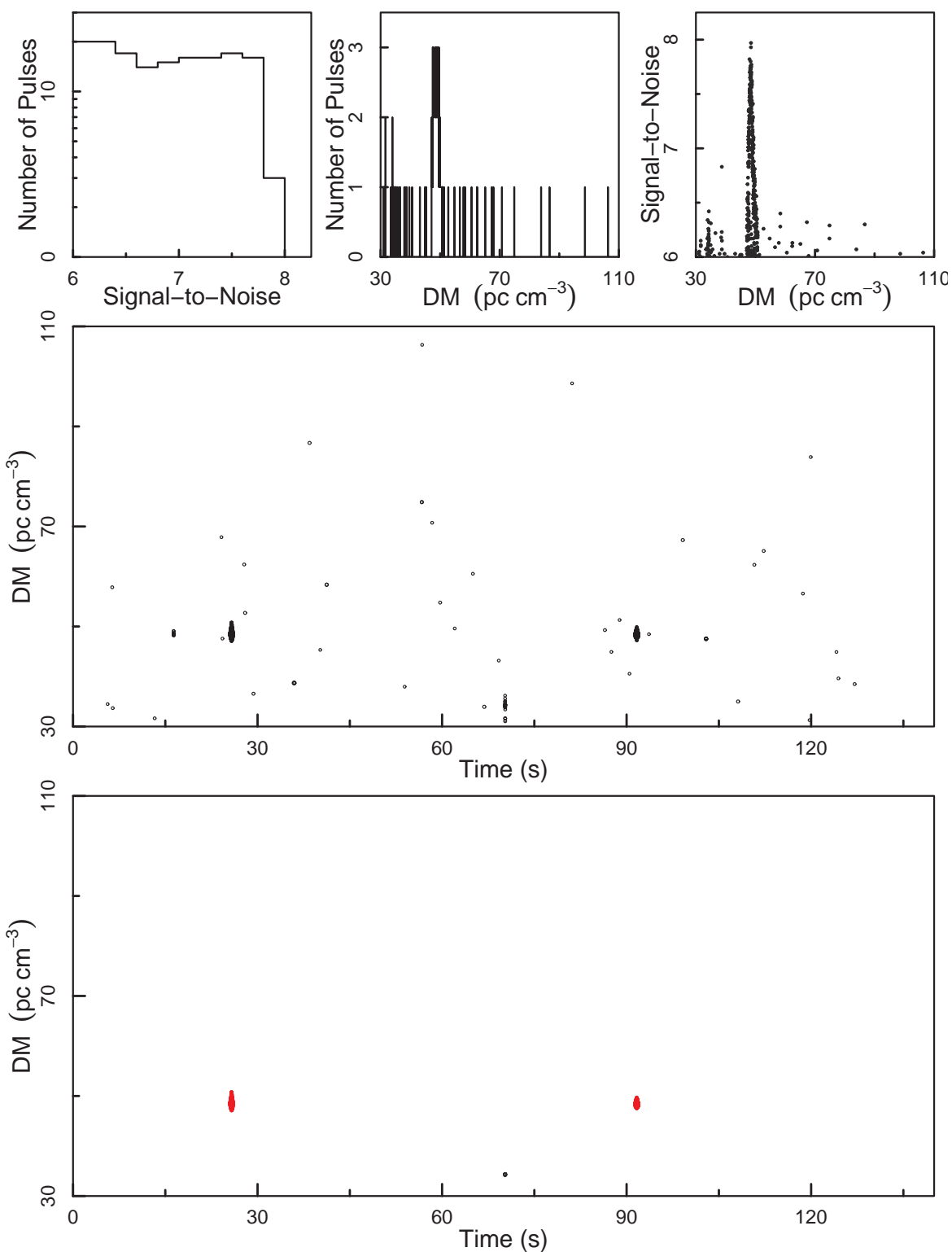

Figure 4.24: Discovery of a pulsar J0539+0007 from the GBTDrift survey. The two SPEGs plotted in red in the bottom subplot are pulses emitted from this pulsar.

DM. These different time and DM extents require different cluster radii. On the one hand, for dim and/or narrow pulses which usually have a small time and DM extent, including any noise event would have a more significant influence on the overall features of the resultant SPEG, necessitating a smaller radius. Furthermore, a small radius is also required to separate pulses that are detected closely in time; on the other hand, because bright, wide pulses will be detected over larger time and DM extents than their dim, 
narrow counterparts, a larger radius is needed to overcome possible large time difference between adjacent single-pulse events; (ii) Data processing techniques can also change the distance between single-pulse events. For example, clipping can lead to larger distances between remaining single-pulse events; furthermore, the distance likely varies with DM. To address these challenges, we used an adequately small radius and introduced the merging of clusters thereafter.

Note that DBSCAN clustering allowed us to find the single-pulse events that were used to calculate the expected DM and time span in the merging step. Moreover, when we used equations (4.1) and (4.2) to calculate the expected DM and time span, we faced the discrepancy between the theoretical $\mathrm{S} / \mathrm{N}$ decline and the observed $\mathrm{S} / \mathrm{N}$ decline, and the problem of clipped pulses as well. We solved these two challenges by using empirical formulas (equations (4.4) and (4.5)) to calculate $\mathrm{S}_{\text {th }}$ (instead of using a fixed value of 5) and obtained satisfactory expected DM and time ranges. Thus both DBSCAN clustering and merging the resultant clusters are necessary for the identification of SPEGs.

Furthermore, SPEGs may vary significantly in brightness, width and shapes. In order to identify the peak-like shape within SPEGs in the S/N versus DM space, we developed a new peak scoring algorithm. Using a low, DM-spacing-dependent threshold for the Fit-Line-Slope allowed us to detect wide and/or dim pulses, as well as pulses of various shapes. However, this also increased the number of non-astrophysical SPEGs.

On the computational side, although DBSCAN clustering, merging the clusters, and peak scoring combined resulted in a significant denoising effect in the DM versus time space, the number of SPEGs that needed to be classified was still high. Therefore we defined the noise level for each beam and only classified relatively bright SPEGs. Furthermore, we excluded the $\chi^{2}$ of the $\mathrm{S} / \mathrm{N}$ versus DM from our feature list because the fitting takes a long time and does not always converge for every pulse, making it impractical to use on large data sets.

In this chapter we selected RandomForest with SMOTE treatment as the best learner because of its very high recall and precision, and very low FPR. It should be noted that all six classifiers had over 93\% recall when SMOTE treatment was applied, but the other five classifiers had significantly lower precision and higher FPR. RandomForest showed the highest precision and lowest FPR most likely because, compared with other learners, it reduced the problem of overfitting. The fact that RandomForest with SMOTE was also selected as the best learner for the classification stage in previous study [26] (which was based on the GBTDrift survey) provides some generalizability of the finding 
that RandomForest in combination with SMOTE performs well for automated pulsar classification. Testing the learners considered in this work and other additional learners on different surveys would help further exploring the generalizability this finding.

A close examination of our results based on the benchmark data set provides some ideas for further improvement of the classification performance. We found that three of the four pulsars missed by RandomForest with SMOTE treatment had less than five fairly dim pulses detected, and the fourth pulsar had a very low DM. The inductive learning hypothesis states that "any hypothesis found to approximate the target function well over a sufficiently large set of training examples will also approximate the target function well over other unobserved examples" [72]. This suggests that our current benchmark data set does not contain enough dim pulsars. In order to improve the classifier's ability to discover dim pulsars, more dim pulsars (once they are discovered), or marginal detections of bright pulsars (once they are found, as those shown in Figs. 4.21 and 4.23), should be included into the benchmark data set and used for training. This agrees with the generally accepted notion that machine learning is not one-shot process of building a data set and applying a learner, but rather an iterative process that includes applying the learner, analysing the results, modifying the data and/or the learner, and repeating [29]. Therefore, we believe that the results can further be improved by creating a more representative benchmark data set, constructing more distinctive features, conducting more hyperparameter optimization, and so on.

The fact that we used periodicity to confirm the astrophysical nature of pulses when three or more pulses were detected at the same DM was helpful for detection of pulsar candidates consisting of a series of fairly dim pulses. However, it remains challenging to discriminate faint, isolated pulses from noise.

Finally, it is important to emphasize that SPEGID is applicable to pulsar surveys of different observational setups, as long as the survey-specific parameters (i.e., the central observing frequency $(\nu)$ and the total bandwidth $(\Delta \nu))$ are modified accordingly. For example, although in this chapter we only presented the results based on data from the PALFA survey, we also tested SPEGID on data from GBT Drift-scan (GBTDrift) survey (without changing any of the parameters except for $\nu$ and $\Delta \nu$ ) and received similar performance. All one needs is to run the procedures of stages 1 and 2 on the survey in hand, preferably using a benchmark data set with pulsar examples from the same survey to train the learner. However, as mentioned earlier, the values of parameters to some degree depend on the research goals. For that reason, parameters' values may 
be slightly modified, whereas changing the forms of equations (4.1), (4.2), (4.4) and (4.5) is not necessary.

\subsection{Conclusion}

In this chapter, we presented a novel, automated two stage single-pulse search approach that improves the speed and accuracy of single-pulse search analysis. In the first stage, SPEGID identified astrophysical pulse candidates as SPEGs through clustering the single-pulse events and merging the clusters. In the second stage, we used supervised machine learning approach to classify SPEGs to pulsar and non-pulsar classes. For that purpose, a benchmark data set containing 60 representative pulsars (detected in 90 beams) was created. Our machine learning experiments on the benchmark data set showed that the classifier based on RandomForest with SMOTE imbalance treatment have the best performance with respect to F-measure. We also discovered a new pulsar from the GBTDrift survey data using the approach described in this chapter. 


\section{Chapter 5}

\section{Pulsar Classification Using Boosting and Deep Learning}

In this chapter, we further investigate the classification stage of the single-pulse search approach SPEGID. Specifically, boosting and deep learning are used in singlepulse searches for the first time, with previous best approach RandomForest used as the baseline learner. We compare the performance of these three learners on benchmark data sets from two different pulsar surveys, namely the GBTDrift survey and the PALFA survey ${ }^{1}$.

\subsection{Feature Extraction Extended}

In Chapter 4, we extracted features from individual SPEGs, and also considered the relation among SPEGs by horizontally grouping them based on their peak DM because pulses that peak at a close DM are likely emitted from the same object. In this chapter, we also group SPEGs vertically. This is because RFI, which typically (but not always) $[12,84]$ peaks at zero DM, can extend over a wide range of DMs; therefore, checking whether there is any brighter SPEG detected at lower DM and similar time helps to distinguish RFI from astrophysical signals, which is commonly used in the process of manual inspection. The extended set of features extracted from SPEGs are listed in Table 5.1. with the five newly added features examining the existence of SPEGs at similar time but lower DM.

\footnotetext{
${ }^{1}$ The work presented in this chapter was published in [80].
} 
Table 5.1: Additional Features extracted for each SPEG used by machine learning algorithms for classification.

\begin{tabular}{lll}
\hline \multicolumn{2}{c}{ Feature } & Description \\
\hline 19 & $S P E G s_{\mathrm{RFI}}$ & Number of SPEGs appearing at close time and lower DM. \\
20 & minDM $_{\mathrm{RFI}}$ & Minimum DM of $S P E G s_{\mathrm{RFI}}$. \\
21 & $S P E G s_{\mathrm{BrRFI}}$ & Number of brighter SPEGs appearing at close time and lower DM. \\
22 & peakDM $M_{\mathrm{BrRFI}}$ & Peak DM of the brightest $S P E G s_{\mathrm{BrRFI}}$ \\
23 & peakS $/ N_{\mathrm{BrRFI}}$ & Peak S/N of the brightest $S P E G s_{\mathrm{BrRFI}}$. \\
\hline
\end{tabular}

\subsection{Creating Two Benchmark Data Sets}

To build pulsar classification models using supervised machine learning and to investigate transfer learning across two different surveys, we created two fully labeled benchmark data sets: one from the GBTDrift survey and another from the PALFA survey. The differences between the two surveys (GBTDrift and PALFA), from which the two benchmark data sets were derived, can be summarized as follows:

- The PALFA survey was conducted at $1.4 \mathrm{GHz}$, whereas the GBTDrift survey was conducted at $350 \mathrm{MHz}$. It is known that pulsar flux densities are generally higher at lower frequencies, but at these frequencies, pulsar survey sensitivity is also reduced by the dispersion smearing, higher sky temperature, and scattering. Therefore, while the GBTDrift survey was optimized for finding bright, nearby pulsars with low observing frequency, most recent large-scale pulsar surveys, including the PALFA survey, are conducted at around $1.4 \mathrm{GHz}[8]$.

- The telescope gain with the ALFA system is higher than that of the GBT. Specifically, the gains of the Arecibo Observatory are $\sim 10 \mathrm{~K}$ and $\sim 8 \mathrm{~K}$, whereas the telescope gain of GBT is only $\sim 2 \mathrm{~K} \mathrm{Jy}^{-1}[61]$.

- Observations conducted in the PALFA survey had a much higher bandwidth (322 $\mathrm{MHz}$ ) than those conducted in the GBTDrift survey (50 MHz).

- The RFI environment at Arecibo is much worse than that at the GBT, which sits within the National Radio Quiet Zone. The GBTDrift survey was conducted in northern summer of 2007 when the azimuth track of the GBT underwent a repair. During this survey, the observations were made when the sky drifted through the telescope beam. Although there was significant on site construction, it has 
been shown that the GBTDrift survey data was not significantly affected by RFI contamination [61].

The summary info of the two benchmark data sets is given in Table 5.2. The name PALFA-extended indicates that we carefully included more dim pulsars into the benchmark data set that were in the previous PALFA benchmark data set [79]. From here on in this proposal, unless explicitly specified, we use GBTDrift and PALFA-exteneded to refer to the two benchmark data sets, not the two surveys. For each benchmark data set, we selected the same number of beams with astrophysical SPEGs and beams without astrophysical SPEGs (i.e., 120 and 1,200, respectively). To ensure that pulsars in each benchmark data set are representative of the pulsar population in the corresponding survey, we selected pulsars with a wide range of DM and $\mathrm{S} / \mathrm{N}$ values. Specifically, the DM of pulsars in GBTDrift ranged from 8 to $245 \mathrm{pc} \mathrm{cm}^{-3}$, and the peak $\mathrm{S} / \mathrm{N}$ of the brightest pulse ranged from 6.3 to 111. Similarly, the DM of pulsars in PALFA-extended ranged from 8 to $614 \mathrm{pc} \mathrm{cm}^{-3}$, and the peak $\mathrm{S} / \mathrm{N}$ of the brightest pulse ranged from 6.5 to 79 .

Table 5.2: The number of beams with and without a pulsar and the number of SPEGs identified within them for the GBTDrift and PALFA-extended benchmark data sets.

\begin{tabular}{|l|r|r|}
\hline Classification Candidates & GBTDrift & PALFA-extended \\
\hline Beams with pulsar & 120 & 120 \\
\hline Beams without pulsar & 1,200 & 1,200 \\
\hline Astrophysical SPEGs & 4,306 & 10,898 \\
\hline Non-astrophysical SPEGs & 46,624 & 230,549 \\
\hline
\end{tabular}

On average, 39 SPEGs were found in a GBTDrift beam, while 183 SPEGs were found in a PALFA beam. Because the observation length of a PALFA beam is only twice as long as that of a GBTDrift beam, this indicates the PALFA beam is generally more noisy and/or has more RFI. Note that the astrophysical and non-astrophysical SPEGs are the instances that are classified by the models (i.e., used for training and testing of the learners). All positive (i.e., astrophysical) and negative (i.e., non-astrophysical) instances were manually labeled when these two benchmark data sets were created.

In the following section, we describe within-survey SPEG classification using two different machine learning techniques: AdaBoost and deep learning. RandomForest, combined with SMOTE treatment to overcome the problem of class imbalance, was 
identified as the best learner in our previous chapter as it had the highest $\mathrm{F}_{1}$ measure [79] and therefore is used as the baseline method in this chapter.

\subsection{SPEG Classification Using AdaBoost}

Boosting is a machine learning approach built on the idea of creating a highly accurate prediction rule by combining many relatively weak and inaccurate rules. As an ensemble method, boosting improves the learning of a given algorithm by training weak learners sequentially, with each trying to correct its predecessor. AdaBoost is the first practical boosting algorithm developed by Freund and Schapire [37]. It boosts the accuracy of a weak learner through carefully adjusting the weights of training instances. If an instance is correctly classified, its weight will be decreased; otherwise its weight will be increased. Therefore, training instances that are hard to classify will become more important in the next iteration. In other words, boosting alters the distribution of the training data by changing the weights of the examples; hence it could be considered as a type of advanced sampling technique [105]. The final output is a weighted majority hypothesis in which the weight of each weak hypothesis is a function of its accuracy, and the overall generalization error will be significantly decreased.

At first sight, applying cost-sensitive AdaBoost variants to SPEG classification seems to be appropriate as false negatives are considered much more problematic than false positives. However, research has shown that the use of the original AdaBoost algorithm with a shifted decision threshold and calibrated probability estimates is preferred [75]. Therefore, in this chapter, we used the original AdaBoost algorithm with the Classification and Regression Trees (CART) as the base learner. As for class imbalance treatment, we found that AdaBoost worked best when it was combined with RUS treatment of the majority class.

\subsection{SPEG Classification Using Deep Learning}

Deep learning adopts the hierarchy of concepts (i.e., a concept is defined in terms of its relation to simpler concept), therefore allows the learning of complicated concepts by building them out of simpler ones. Unlike most machine learning algorithms that are capable of discovering the mapping from features to output, deep learning solves the central problem in representation learning by introducing representations that are expressed in terms of other, simpler representations [41]. This way, deep learning is good 
at identifying the factors of variation that explain the observed data and hence alleviates the significance of feature design and is also robust to noise. In [79] we applied multilayer perceptrons (MLPs), a type of feedforward neural networks, on SPEG classification. The trained model performed poorly compared to the RandomForest model. We believe that MLPs did not perform well because we used a shallow neural network, with only one hidden layer.

In this work, we develop deep feedforward neural networks with different numbers of layers and hidden units, selected the one that performs the best, and named it SPEGnet. Specifically, for the GBTDrift benchmark data set we found that the optimal neural network architecture had four hidden layers, with 200, 100, 50, and 20 hidden units respectively, and with a dropout rate of 0.3. The whole network was trained for 50 epochs with a learning rate of 0.0001. For the PALFA-extended benchmark data set, to achieve similar performance, three more layers had to be added to the network. The resultant network therefore had seven hidden layers, with 200, 200, 100, 100, 50, 50 and 20 hidden units respectively, but the optimal training parameters remained unchanged. For both data sets, adding more layers increased the computing complexity significantly, but does not further improve the learning performance. Like RandomForest, SPEGnet worked best when combined with the SMOTE treatment of the minority class.

\subsection{Hyperparameters Tuning}

The hyperparameters were tuned by dividing the 1320 beams into 6 stratified folds (each fold contains 20 beams with known pulsars and 200 beams with no pulsars detected) and then using nested 6 -fold cross-validation. The outer loop ran over all 6 folds with one fold reserved for testing, and the inner loop performed a standard 5-fold cross-validation in order to select the best model hyperparameters [67].

\subsection{Performance Metrics}

Generally speaking, the most commonly used performance metrics for pulsar searching models are Recall, Precision, FPR, F-measure and G-Mean (which is defined as the geometric mean of Recall and Specificity $\left.{ }^{2}\right)[73,63,6,17]$. Due to the inherent class imbalance of pulsar searching data, metrics that depend on the imbalance ratio of positive to negative examples in the test data, such as Accuracy, are not suitable for

\footnotetext{
${ }^{2} \mathrm{G}-\mathrm{mean}=\sqrt{\frac{\mathrm{TP}}{\mathrm{TP}+\mathrm{FN}} \times \frac{\mathrm{TN}}{\mathrm{TN}+\mathrm{FP}}}$
} 
comparing classifier performances [73]. In contrast, as shown in Equation (2.3), Recall is only derived from statistics of the minority (positive) class. While Precision (given with Equation (2.5)) is affected by the number of false positives, it is independent of the number of true negatives (majority class); hence, Precision is not significantly affected by the imbalance ratio of the test data. Consequently, F-measure which is derived from Recall and Precision is commonly used to compare the model performances even when they are trained and tested on data with different imbalance ratios [22]. As shown in Equation (2.6), FPR is proportional to the number of false positives. However, FPR is also inversely proportional to the total number of negative examples within the test data. Therefore it can be more easily affected by the imbalance ratio. If the number of negative samples is very large (as in an highly imbalanced data set), the number of true negatives (TN) in the FPR denominator would probably be very high and make this metric artificially smaller. Note that, periodicity searches and frequency-time searches aimed at discovering FRBs often deal with millions of candidates and therefore require models to have much lower FPR than time-domain single-pulse searches.

In previous chapter, we the used $\mathrm{F}_{1}$ measure, which commonly used as performance metric in many machine learning problems, as the performance metric for pulsar classification models. Due to the strong preference on high recall (i.e., low false negative rate) in pulsar searches, we investigate the general formula of the F-measure to find a better performance metric for pulsar classification models.

In the previous chapter, we used the commonly used $\mathrm{F}_{1}$ measure (defined by Equation 4.12) to select the best pulsar classification model One limitation of $F_{1}$ measure is that it gives equal importance to recall and precision, which is not always desired for extremely skewed data sets. For the full definition of F-measure (also known as $\mathrm{F}_{\beta}$, defined in Equation 2.7), the value of $\beta$ can be adjusted to reflect the relative importance of precision and recall. Given that in pulsar detection a high recall is highly preferred even at the cost of relatively high FPR, in this work we explored different values of $\beta$ and decided that for pulsar classification models $\mathrm{F}_{4}$ measure was a better performance metric than $\mathrm{F}_{1}$ measure.

\subsubsection{Binary Classification versus Probabilistic Prediction}

It is worth pointing out that in many pulsar searching studies $[62,26,63,17,70]$, a binary classification was used to separate pulsar signals from non-pulsar signals. Thus, the models simply assign a label of 0 or 1 to each candidate. The performance metrics 
were also selected based on this idea. Alternative option to binary classification would be to use probabilistic prediction that outputs the confidence of each candidate being astrophysical and use this information to rank the candidates. That way, more credible candidates could be inspected first. In addition, different probability thresholds could be chosen, which would decide the total number of candidates to be inspected by human resources that are always limited in reality. In addition, ROC and Precision-Recall curves can be plotted and used to identity the best model.

\subsection{Classification of SPEGs versus Pulsars}

It is important to note that multiple SPEGs are often observed from the same pulsar during an observation. In fact, the detection of a pulsar is based upon the correct classification of its emitted pulses (i.e., SPEGs). In the most extreme case, only one SPEG needs to be correctly classified in order to detect a pulsar. In other words, for pulsars with multiple observed SPEGs, misclassifying some of the SPEGs does not necessarily result in missing of the pulsar. On the other hand, for pulsars with only a few SPEGs detected, misclassifying even one SPEG significantly increases that probability.

While the machine learning models were trained and tested on SPEGs as mentioned in section 5.2, we report the performance of models based on pulsars (i.e., beams) because they are of much greater practical importance to the astronomers than the performance measured by SPEGs. When comparing these results with results of other works it should be kept in mind that the performance measured by pulsars would generally appear worse than when it is measured by SPEGs.

\subsection{Experimental Results}

In this section, we first show the within-survey classification results using two new models based on AdaBoost and SPEGnet (i.e., the best performing deep feedforward neural network) and compare them with RandomForest (which was the best classifier in the previous chapter) as the baseline model. Then, we present the across surveys prediction, both without and with transfer learning. In the former we used benchmark data from the same survey for both training and testing, while in the latter we treated one benchmark data set as the source and the other as the target. 


\subsubsection{GBTDrift Benchmark Classification}

Table 5.3 and Figure 5.1 show the classification results for GBTDrift. Remember that the classification is done at SPEG level, but if not otherwise specified we report the performance metrics at pulsar level (see Section 5.7). To determine which F-measure is appropriate for the problem of pulsar detection, we calculated F-measures for different $\beta$ values and present the results in Figure 5.1. (Table 5.3 shows the results of training on 100 pulsars, which correspond the rightmost three points in Figure 5.3.)

Table 5.3: Classification results of different models trained on 100 and tested on 20 pulsars from the GBTDrift benchmark data set using 6-fold cross validation.

\begin{tabular}{|l|c|c|c|}
\hline Metric & RandomForest & AdaBoost & SPEGnet \\
\hline Recall (SPEG) & $0.871 \pm 0.001$ & $0.958 \pm 0.004$ & $0.920 \pm 0.004$ \\
\hline Recall & $0.906 \pm 0.001$ & $0.985 \pm 0.001$ & $0.974 \pm 0.002$ \\
\hline FPR & $0.017 \pm 0.002$ & $0.080 \pm 0.001$ & $0.074 \pm 0.002$ \\
\hline$F_{1}$ & $0.872 \pm 0.002$ & $0.708 \pm 0.002$ & $0.717 \pm 0.003$ \\
\hline$F_{4}$ & $0.902 \pm 0.001$ & $0.942 \pm 0.001$ & $0.934 \pm 0.001$ \\
\hline
\end{tabular}

As can be seen from Table 5.3, for GBTDrift, AdaBoost and SPEGnet resulted in significant improvements of the Recall values (at both SPEG and pulsar level) compared to the baseline results produced by RandomForest. Specifically, AdaBoost outperformed SPEGnet and RandomForest, providing around $1.1 \%$ and $7.9 \%$ better Recall values at the pulsar level, respectively. Meanwhile, all three models had FPR smaller than 8\%, which would reduce the number of candidates requiring manual inspection by a factor of over 10. Note again, we report the performance metrics based on beams because they provide a more realistic view for practical use. However, as discussed in subsection 5.7, that leads to higher FPR than when results are reported based on SPEGs. In addition, in our approach only one diagnostic plot is produced for each beam, which further reduces the total number of diagnostic plots compared with the convention of producing multiple diagnostic plots for different DM ranges. Consequently, our approach uses a significantly smaller number of diagnostic plots compared to periodicity searches, and perhaps FRB searches in high RFI environments, for which tens of diagnostic plots are generated for each pointing (or pseudo-pointing) [93]. Keeping in mind that our goal was to find not only the bright pulsars in our data, but also dim pulsars with low $\mathrm{S} / \mathrm{N}$, for our experiments the above mentioned FPR values are acceptable. In general, the acceptable 

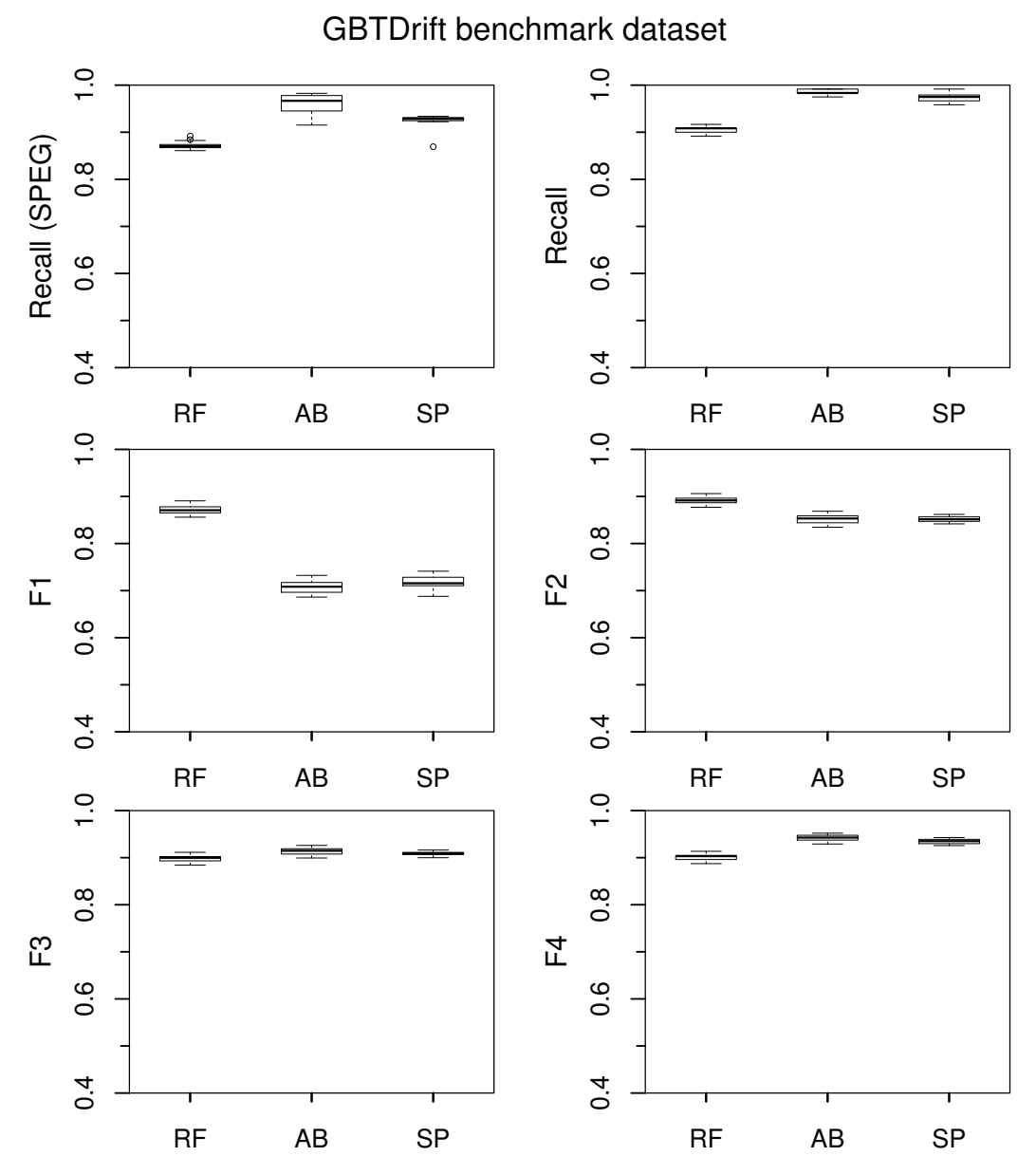

Figure 5.1: Different performance metrics for pulsar classification models trained and tested on GBTDrift. Notation: RandomForest (RF), AdaBoost (AB), SPEGnet (SP). 
FPR will depend on factors such as the data rate, storage capability, RFI environment, person-power available to inspect the candidates, and the time available for candidates' follow-up.

Given our emphasis on high Recall, the AdaBoost model would be considered the best among the three, and the SPEGnet model the second best. However, if measured in terms of $F_{1}$ measure $(\beta=1)$, which gives the same weight to the Recall and Precision, RandomForest would have been considered as the best model, even though it had the lowest Recall. As can be seen in Figure 5.1, $\mathrm{F}_{4}$ measure correctly reflected the importance of high Recall over low FPR, and showed that AdaBoost outperformed SPEGnet, and both outperformed RandomForest. Therefore, we conclude that $\mathrm{F}_{4}$ measure reflects the desired performance in the radio pulsar detection area better than $\mathrm{F}_{1}$ and should be used as the selection criterion for the best performing model.

The AdaBoost model was able to find almost all pulsar beams in the GBTDrift benchmark with high precision, therefore it was selected as the best model. In practice, however, the number of beams collected in a pulsar survey can be tens or even hundreds of times as large as the GBTDrift benchmark. In those cases, limited resources can be assigned to the manual inspection of beams containing positively classified instances. To illustrate the trade-offs between different performance metrics [22], we modified our code to use AdaBoost model to produce probabilistic predictions and calibrated the predicted probabilities using Platt Scaling [75]. The Precision-Recall (PR) and Receiver Operator Characteristic (ROC) curves of AdaBoost model are shown in Figure 5.2. The different Precision, Recall, and FPR values were obtained using different thresholds for predicted probabilities to decide above which SPEGs should be classified as astrophysical. (Note that AdaBoost model predicted the probability of each SPEG being astrophysical, but the curves in Figure 5.2 show the performance metrics based on beams not individual SPEGs.) As can be seen in Figure 5.2, the PR curve is located in the upper-righthand corner, which indicates the good performance of the AdaBoost model. (Previous studies showed that for highly skewed data sets, like pulsar searching data, the PR curve provides a more informative picture of a learner's performance than the ROC curve [22].) In the future, we plan to use probabilistic prediction and compare all models using the PR curve. Note that it is possible for the performance of the three models to be ranked differently at different precision levels.

We also examined how the size of training data affected these three models for GBTDrift, with the results shown in Figure 5.3. Note that the smaller training data sets 

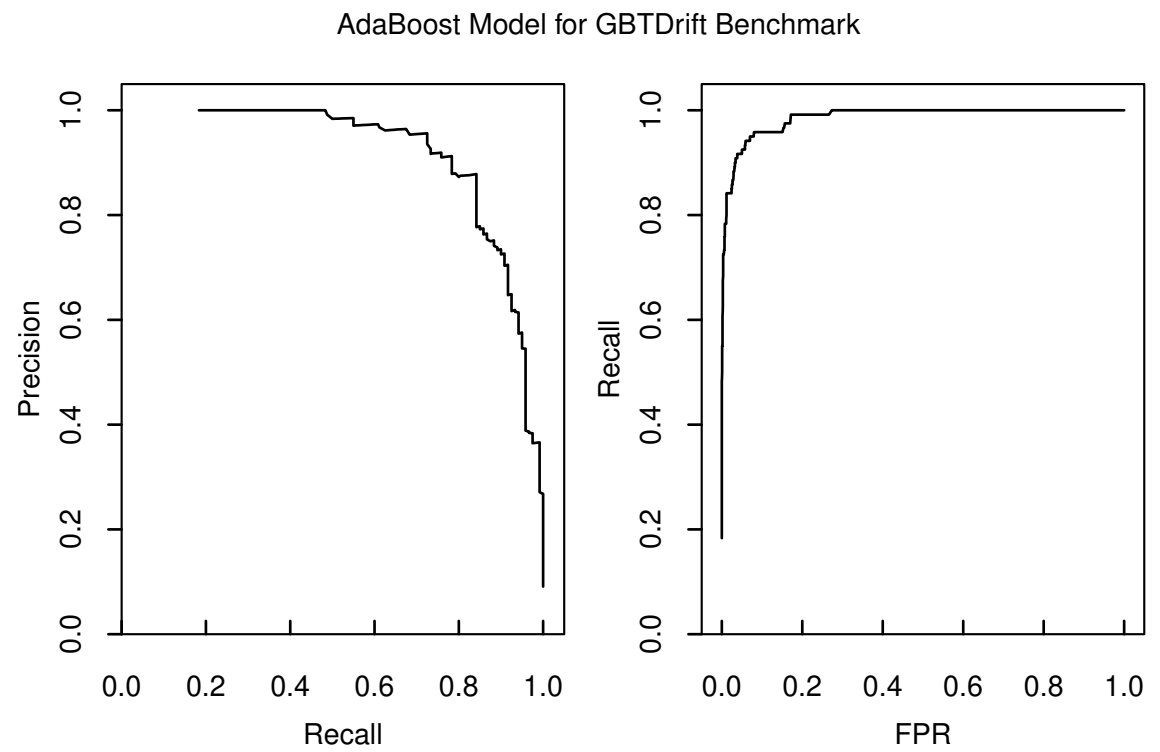

Figure 5.2: The PR and ROC curves of AdaBoost model trained on GBTDrift using 6-fold cross-validation.
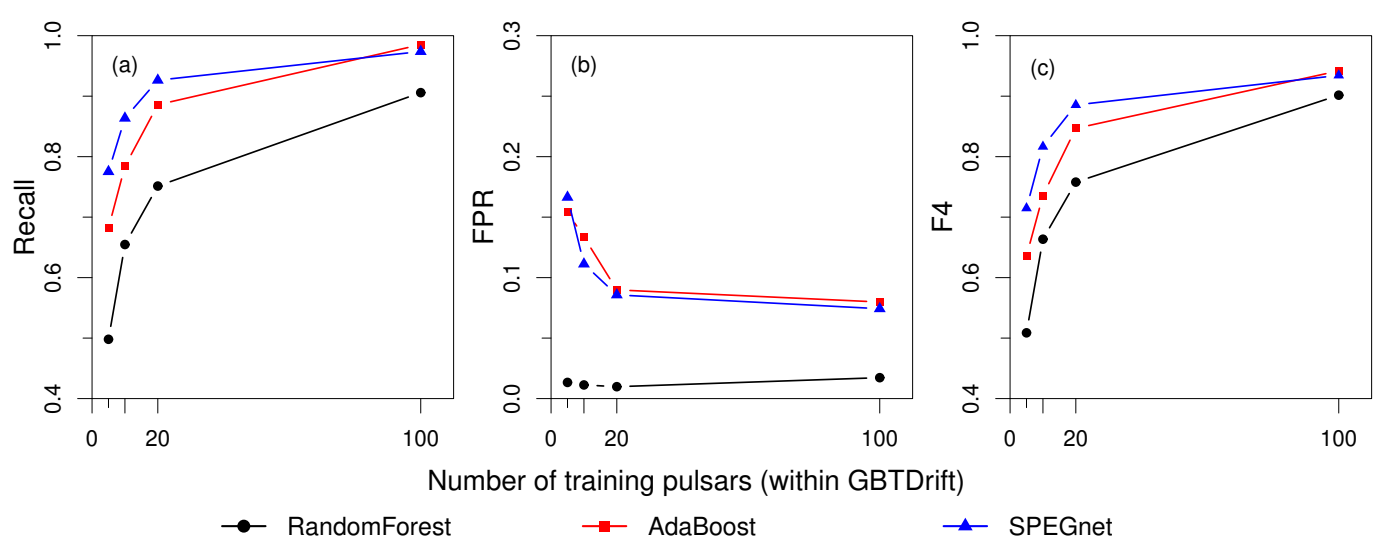

Figure 5.3: Recall, FPR, and $\mathrm{F}_{4}$ measure of three pulsar classification models within GBTDrift, for different sizes of training data. 
were created by randomly sampling from the full benchmark data set and the experiments were repeated 100 times. It can be seen that AdaBoost and SPEGnet models had higher Recall values (Figure 5.3(a)), but also higher FPR values (Figure 5.3(b)) than RandomForest. Nevertheless, the results presented in Figure 5.3(c) showed that the performance of all models measured in term of $\mathrm{F}_{4}$ measure was consistent with when they were measured in terms of Recall. This indicates that the influence of high Recall outweighed the influence of relatively high FPR. As the size of the training data increased, the Recall values of all three models increased. Meanwhile, the FPR values of AdaBoost and SPEGnet models consistently decreased, which shows that both algorithms suffered severely from overfitting when the training data were limited. As more data were included, the problem of overfitting was mitigated. In contrast, the FPR of RandomForest model increased as the algorithm was trained on more data. This shows that RandomForest was less susceptible to overfitting [9] and it generated overly simplistic models when trained on limited training data. When the number of training pulsars reached 20, the SPEGnet model had an $\mathrm{F}_{4}$ measure of 0.915 ; further increase of the amount of training data improved the $\mathrm{F}_{4}$ measure only slightly.

\subsubsection{PALFA-extended Benchmark Classification}

The within-survey classification results for PALFA-extended are shown in Table 5.4 and Figure 5.4. As can be seen in Table 5.4, AdaBoost and SPEGnet models had around $3 \%$ higher Recall, but their FPR was much higher (around 15\%) than the RandowmForest model. It should be noted that including more low $\mathrm{S} / \mathrm{N}$ pulsars into the benchmark data set decreased the Recall of the RandomForest model compared with our previous results [79]. Unlike the GBTDrift results, even when the $\mathrm{F}_{4}$ measure was used as performance metric, RandomForest would still be considered the best model, though the performance differences among the three models were much smaller than when the $\mathrm{F}_{1}$ measure was used for performance evaluation.

The PR and ROC curves of the RandomForest model trained on the PALFAextended benchmark are shown in Figure 5.5. Similarly as in Figure 5.2, the PR curve is located in the upper-right-hand corner, which indicates the good performance of the RandomForest model. Compared with our previous study [79], the introduction of more low $\mathrm{S} / \mathrm{N}$ pulsars into the PALFA-extended benchmark resulted in the decrease of the Recall of RandomForest model. To clearly show how the performance of the RandomForest model was affected by the low S/N pulsars, in Figure 5.6 we present the True Positive, 
Table 5.4: Classification results of different models trained on 100 and tested on 20 pulsars from the PALFA-extended benchmark data set using 6-fold cross validation.

\begin{tabular}{|l|c|c|c|}
\hline Metric & RandomForest & AdaBoost & SPEGnet \\
\hline Recall (SPEG) & $0.906 \pm 0.000$ & $0.894 \pm 0.023$ & $0.948 \pm 0.017$ \\
\hline Recall & $0.916 \pm 0.001$ & $0.945 \pm 0.012$ & $0.948 \pm 0.011$ \\
\hline FPR & $0.058 \pm 0.002$ & $0.158 \pm 0.010$ & $0.145 \pm 0.012$ \\
\hline$F_{1}$ & $0.733 \pm 0.006$ & $0.537 \pm 0.015$ & $0.560 \pm 0.023$ \\
\hline$F_{4}$ & $0.890 \pm 0.001$ & $0.867 \pm 0.001$ & $0.877 \pm 0.001$ \\
\hline
\end{tabular}

PALFA-extended benchmark dataset
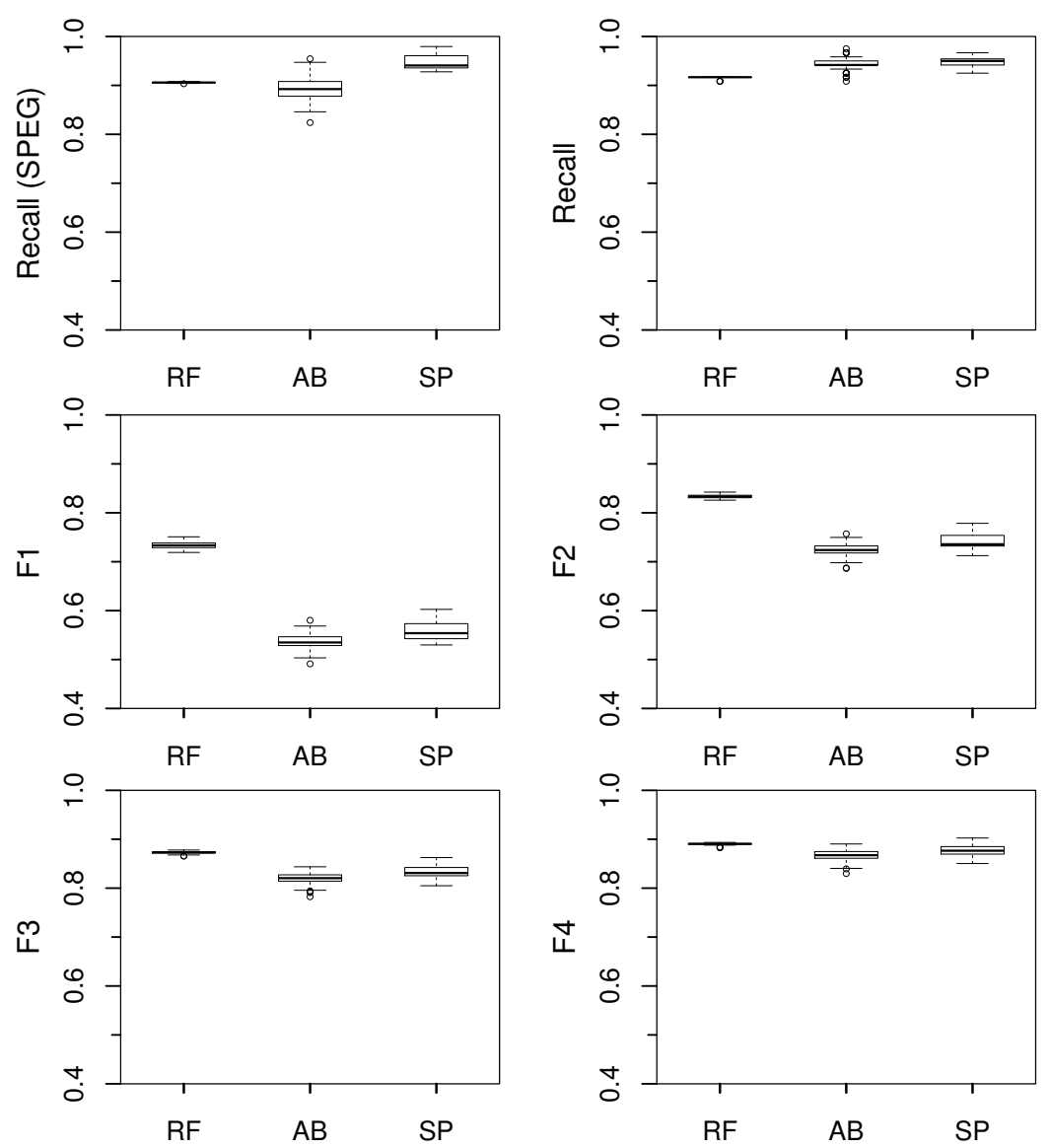

Figure 5.4: Performance metrics for pulsar classification models trained and tested on PALFA-extended. Notation: RandomForest (RF), AdaBoost (AB), SPEGnet (SP). 

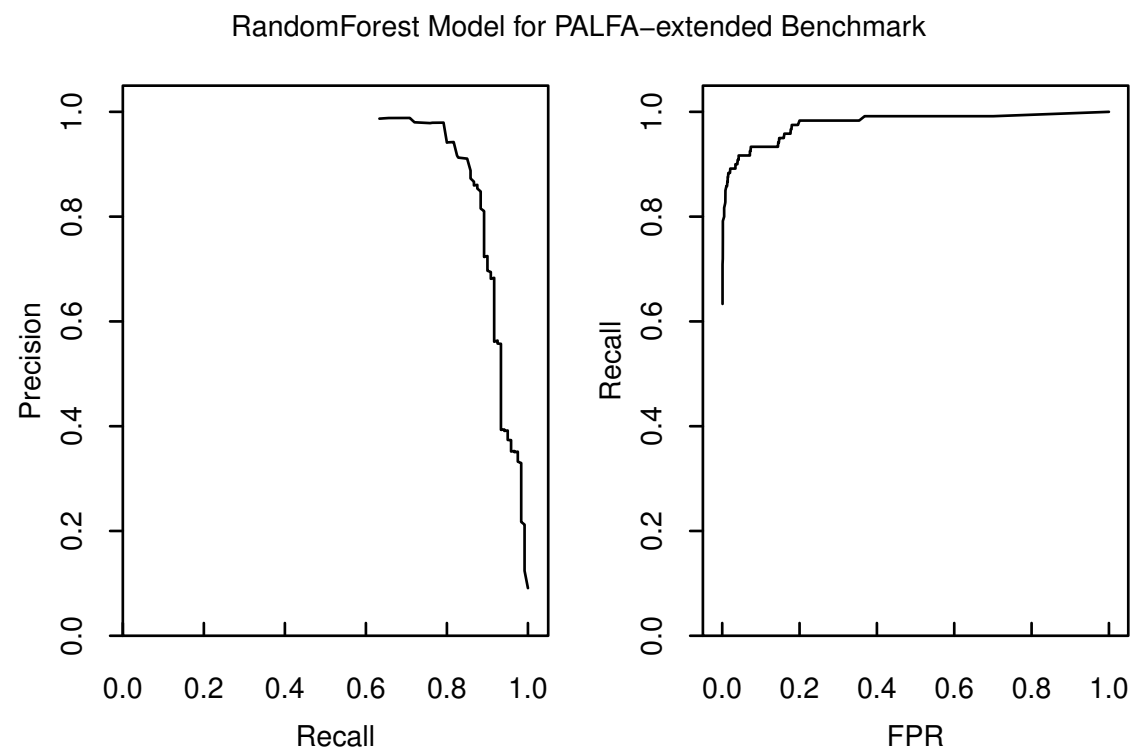

Figure 5.5: PR and ROC curves of RandomForest model trained on PALFA-extended using 6 -fold cross-validation.

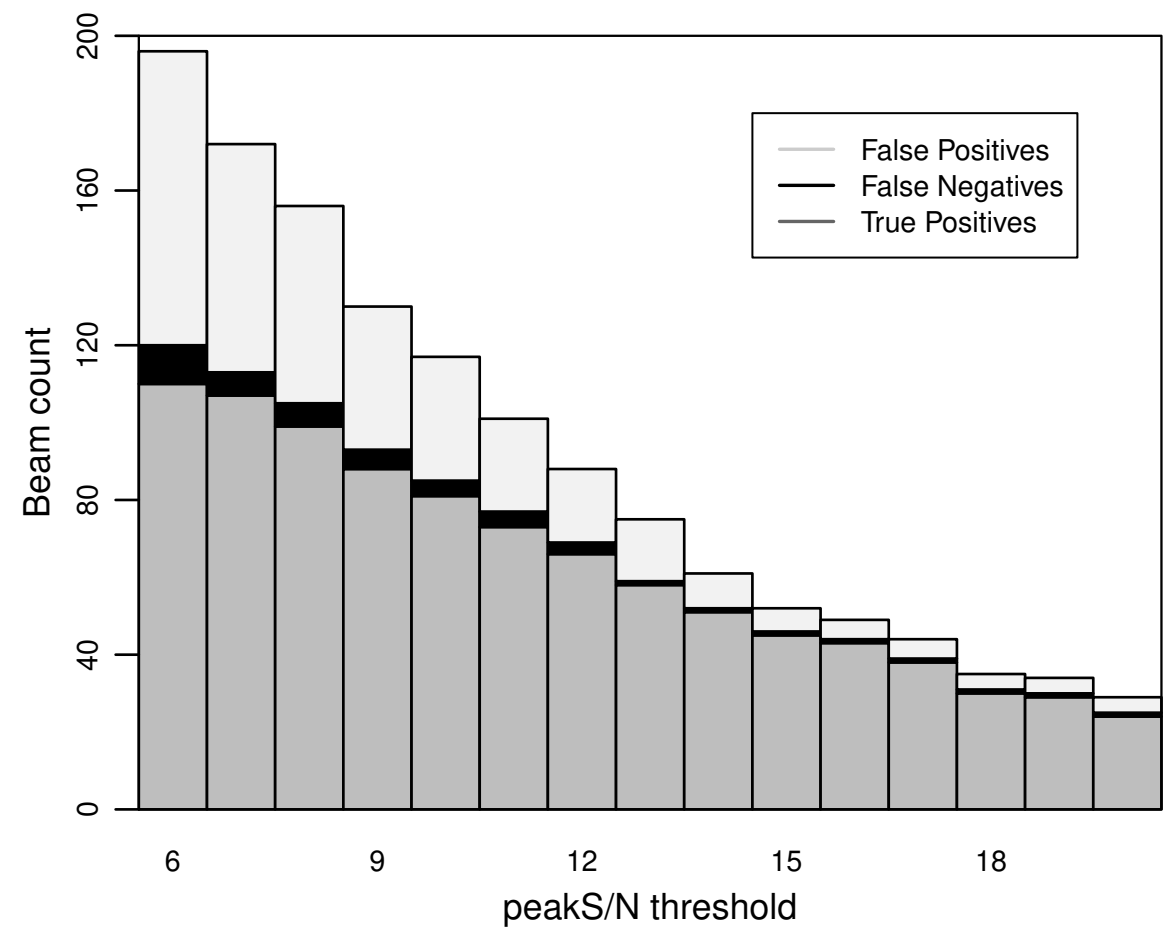

Figure 5.6: True Positive, False Negative, and False Positive values against different peak $S / N$ thresholds for RandomForest model trained and tested on PALFA-extended. 
False Negative, and False Positive values against the "peakS/N" threshold. As can be seen in Figure 5.6, both False Negatives and False Positives increased when the "peak$\mathrm{S} / \mathrm{N}$ " threshold decreased from 7 to 6 . This shows that it is more difficult to separate low $\mathrm{S} / \mathrm{N}$ pulsar signals from RFI and noise.
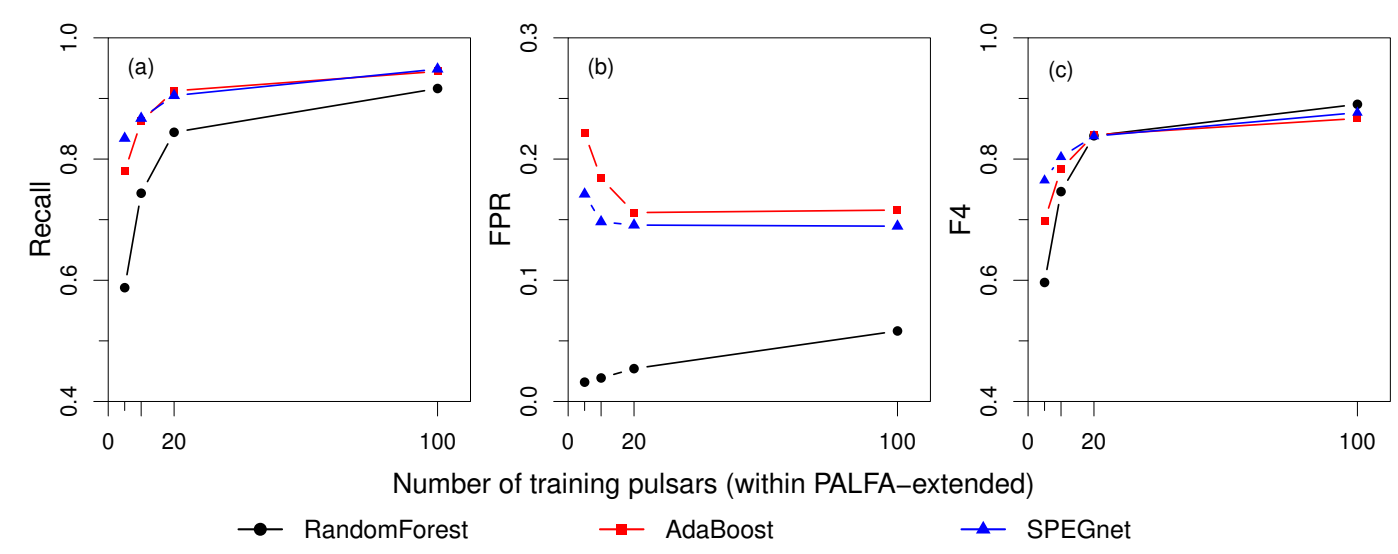

Figure 5.7: Recall, FPR, and $\mathrm{F}_{4}$ measure of pulsar classification models within PALFAextended, for different sizes of training data.

We also examined how the size of training data affected the performance of these three models. As shown in Figure 5.7, the Recall values of AdaBoost and SPEGnet models were consistently higher than those of the RandomForest models (Figure 5.7(a)), but they also had much higher FPR (Figure 5.7(b)). As the size of the training data increased, the Recall of all three models increased, and the FPR of AdaBoost and SPEGnet decreased while the FPR of RandomForest increased slightly. As can be seen in Figure 5.7(c), the $\mathrm{F}_{4}$ measure of all models improved with increasing the amount of training data. When the training data were limited to less than 20 pulsars, SPEGnet outperformed AdaBoost, and both outperformed RandomForest. When the number of training pulsars reached 20, the performance (measured in terms of $\mathrm{F}_{4}$ ) of all three models was very similar. Further increase of the amount of training data improved the $\mathrm{F}_{4}$ measure only slightly. (Note that Table 5.4 and Figure 5.4 show the results of training on 100 pulsars, corresponding to the rightmost three points in Figure 5.7.)

Compared with the within GBTDrift classification results, the PALFA-extended classification results generally had lower Recall, higher FPR and thus lower $\mathrm{F}_{4}$ measures. 


\subsection{Conclusion}

We found that compared to the $\mathrm{F}_{1}$ measure, commonly used as performance metric in many machine learning problems, the $\mathrm{F}_{4}$ measure was a better performance metric for pulsar classification models due to the strong preference on high recall (i.e., low false negative rate). In general, depending on the data set (i.e., survey) used and the amount of training data, different models performed the best. Compared with the within GBTDrift classification results, the PALFA-extended classification results generally showed lower recall, higher FPR and hence lower $\mathrm{F}_{4}$ measures. 


\section{Chapter 6}

\section{Discovery of Seven Pulsars from the AO327 Survey Using SPEGID}

In the previous chapter, we showed that the ability of our pulsar searching approach to detect low $\mathrm{S} / \mathrm{N}$ pulsars in the data was improved due to the following two reasons: (i) including enough low $\mathrm{S} / \mathrm{N}$ pulsars in the benchmark data; (ii) predicting the probability of each candidate being astrophysical and using a low probability threshold for candidates that will be manually inspected. Because we obtained good results on our benchmark data (especially on the GBTDrift benchmark), we decided to apply our single-pulse search approach to more real data and further test its ability of finding pulsars in other pulsar surveys.

\subsection{AO327 Drift Pulsar Survey Description}

In this chapter, we use single-pulsar search data from the Arecibo $327 \mathrm{MHz}$ (AO327) drift pulsar survey. The AO327 survey is a filler project as it collects data whenever the Arecibo telescope time becomes available, e.g., when scheduled observation cannot be conducted due to technical problems [25]. It collects data over the entire Arecibo sky (with the declination between -1 and 38 degree). A lower frequency than the PALFA survey was selected because pulsars often exhibit a power-law spectra such that their signals decrease significantly as the frequency increases $(\mathrm{S} \propto \alpha$, with average $\alpha=-1.7$ [25]). The AO327 survey started collecting data in 2010 when the telescope platform was under repair and the telescope movement was restricted. The observing bandwidth was $57 \mathrm{MHz}$ using the Mock spectrometer. At $327 \mathrm{MHz}$, the AO beam has a width of $15^{\prime}$ and therefore has a maximum transit time of 1 minute. As a result, each beam is processed to 
have a span of $60 \mathrm{~s}$, with $30 \mathrm{~s}$ overlapping between successive beams. At the dedispersion stage, 6,358 trial DM values between 0 and $1095 \mathrm{pc} \mathrm{cm}^{-3}$ were used. Single-pulse search data were generated using the PRESTO pipeline [89]. All single-pulse events with S/N $\geq 5$ were saved.

\subsection{Clusterrank}

Single-pulse search results for AO327 were first analyzed using a single-pulse search algorithm called "Clusterrank", which resulted in discovery of 22 pulsars and RRATs until 2016 [24]. Clusterrank first identifies pulse candidates using predetermined DM and time gaps. If the DM and time differences between two single pulses are smaller than those predetermined gaps, they are considered as belonging to the same cluster. Clusterrank selects $1 \mathrm{pc} \mathrm{cm}^{-3}$ as the maximum DM gap (which was the largest DM spacing between adjacent trial DM values.), and $19 \mathrm{~ms}$ as the maximum time gap. If there are at least 50 events in the final cluster, then the cluster will be considered as a valid pulse candidate. Clusterrank generates four diagnostic plots with different DM ranges. After that, Clusterrank applies least-square fitting to the $\mathrm{S} / \mathrm{N}$ vs DM curve based on the theoretical Gaussian shape of an astrophysical pulse [19] (see Equations 4.1 and 4.2).

These equations show how an increased offset in DM causes a decrease in the observed $\mathrm{S} / \mathrm{N}$. Next, $\mathrm{R}^{2}$ was used as the score measuring the goodness of fit [51], which was considered a better test statistic than the reduced $\chi^{2}$ or the root mean square residual of the least-squares fit. The diagnostic plots are examined based on the highest $\mathrm{R}^{2}$ value of the clusters in descending order. If a diagnostic plot does not contain any candidate with $\mathrm{R}^{2}$ value of at least 0.8 , it will then be ignored. To reduce the number of RFI candidates, Clusterrank also set the score of a candidate to 0 if the best-fit pulse width is negative or if the best-fit DM is less than $1 \mathrm{pc} \mathrm{cm}^{-3}$. For beams with many candidates, to reduce the number of false positives, a modification of the Bonferroni correction to the familywise error rate was used. The Bonferroni correction adjusts probability values caused by the increased risk of a type I error when making multiple statistical tests. More details can be found in [3].

\subsubsection{Clusterrank versus SPEGID}

Compared with RRATtrap [50], Clusterrank is able to detect pulses that are clipped into two separate parts during the RFI removal stage. However, Clusterrank could result 
in false negatives belonging to three types [24]. The first type are pulses that produced a cluster of less than 50 events. This is often observed in pulses detected at high DMs because the DM spacing between adjacent DM channels increases as DM increases in PRESTO. Moreover, this may also be problematic for very narrow and/or dim pulses as they usually are only detected in a few DM channels. The second type is a pulse fitted with a score less than 0.8 , which has been observed in pulses with multiple peaks. The third type is caused by the Bonferroni correction when many RFI cluster are present in the observed beam.

In contrast, in our single-pulse search approach SPEGID, we set the threshold for the number of events in a pulse candidate must have to five. Consequently, our approach is able to identify both narrow and dim pulse candidates. Furthermore, we extract a series of features from the pulse candidates and use supervised machine learning models to classify them instead of using a single test statistic. Therefore, our pulsar classification models are able to find pulses that may have different characteristics than regular pulses such as very narrow pulses, pulses with more than one component, etc. Additionally, our approach considers the association between candidates that are detected at a close DM, which has been proved beneficial for finding dim pulsars with multiple pulses detected. To evaluate how our pulsar searching approach would perform on real data, we used our two-stage single-pulse search approach SPEGID to re-analyze the AO327 survey data.

\subsection{Results}

The data set we analyzed contains 297,674 beams observed between November 2013 to June 2019. It has in total 297,000 GB of single-pulse search files generated by PRESTO. The observation frequency $\left(f_{\text {center }}\right)$ and bandwidth $(\Delta \nu)$ are really close to the GBT Drift scan survey (GBTDrift), as shown in Table. 6.1. Therefore, instead of creating a new benchmark data from AO327 data, we decide to use pulsar classification model trained the GBTDrift benchmark data to classify candidates identified in the AO327 data. We decide to use AdaBoost model as it outperformed the other two models on the GBTDrift benchmark data.

We found that our model was not only able to find all pulsars that had previously been discovered by Clusterrank, but also found 80 candidates for follow-up observations. After the re-observation, seven candidates were confirmed as pulsars (or RRATs). These pulsars newly discovered by our approach can be grouped into the following three categories: 


\begin{tabular}{lrr}
\hline \hline Survey & AO327 & GBT350 \\
\hline$f_{\text {center }}(\mathrm{MHz})$ & 327 & 350 \\
$\Delta \nu(\mathrm{MHz})$ & 57 & 50 \\
\hline
\end{tabular}

Table 6.1: Comparison of the observing frequency and bandwidth between AO327 survey and GBT350 survey.

- Pulsars with one narrow but relatively bright pulse observed (with peak S/N between 7.5 and 9.5), as shown in Figures 6.1, 6.2, 6.3;

- Pulsars that have multiple dim pulses with an underlying periodicity (with peak $\mathrm{S} / \mathrm{N}$ between 6 and 7.5) detected at close DM, as shown in Figures 6.4, 6.5, 6.6;

- Pulsars with multiple relatively bright pulses observed (with maximum peak S/N greater than 9) in a beam heavily contaminated by RFI, as shown in Figure 6.7.

Furthermore, we also found one pulsar (J2027+02, shown in Figure 6.8) in our data that had been missed by Clusterank but was later discovered in another pulsar survey before our re-analysis. Therefore, it could no longer be classified as discovery but rather "redetection".

\subsection{Conclusion}

The results prove that our pulsar searching approach is able to find pulsars that are often difficult to find by both automated detection and manual inspection. We expect including more negative training examples such as RFI from the AO327 survey will likely further reduce the number of false positives. On the other hand, due to the unfortunate collapse of the Arecibo telescope, it becomes difficult to carry out continued follow-up observation. Nonetheless, the Five hundred meter Aperture Spherical Telescope (FAST), which became fully operational in 2020, enables the measurement of timing-derived parameters and better constraints on emission mechanisms that will allow us to learn how these objects relate to other pulsars and what is responsible for their unusual emission. 


\section{p1693.Strip43.00100.D032758+0935_0001 DM: 93.68 Period: NA S}
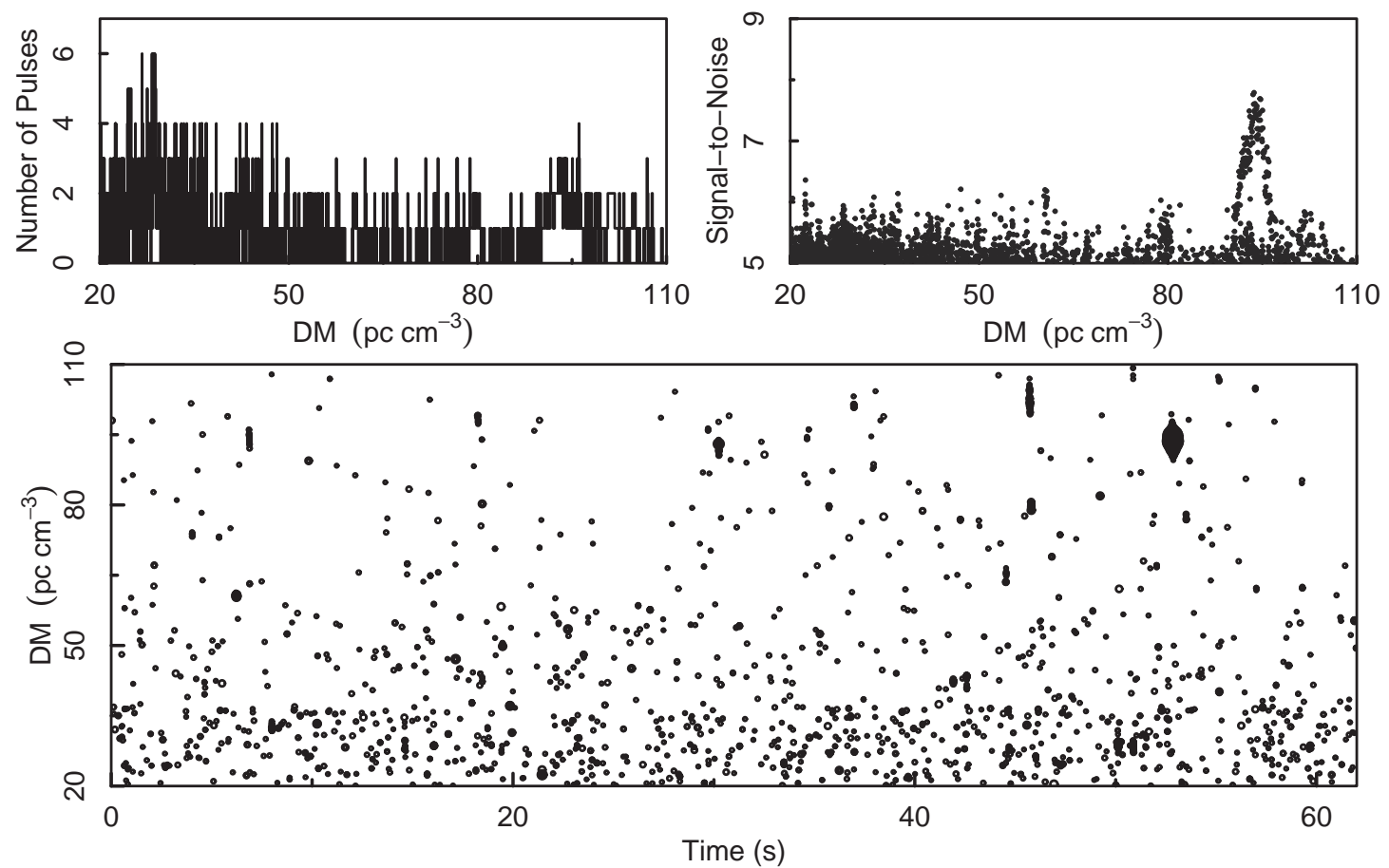

Pulse Candidates Identified by SPEGID
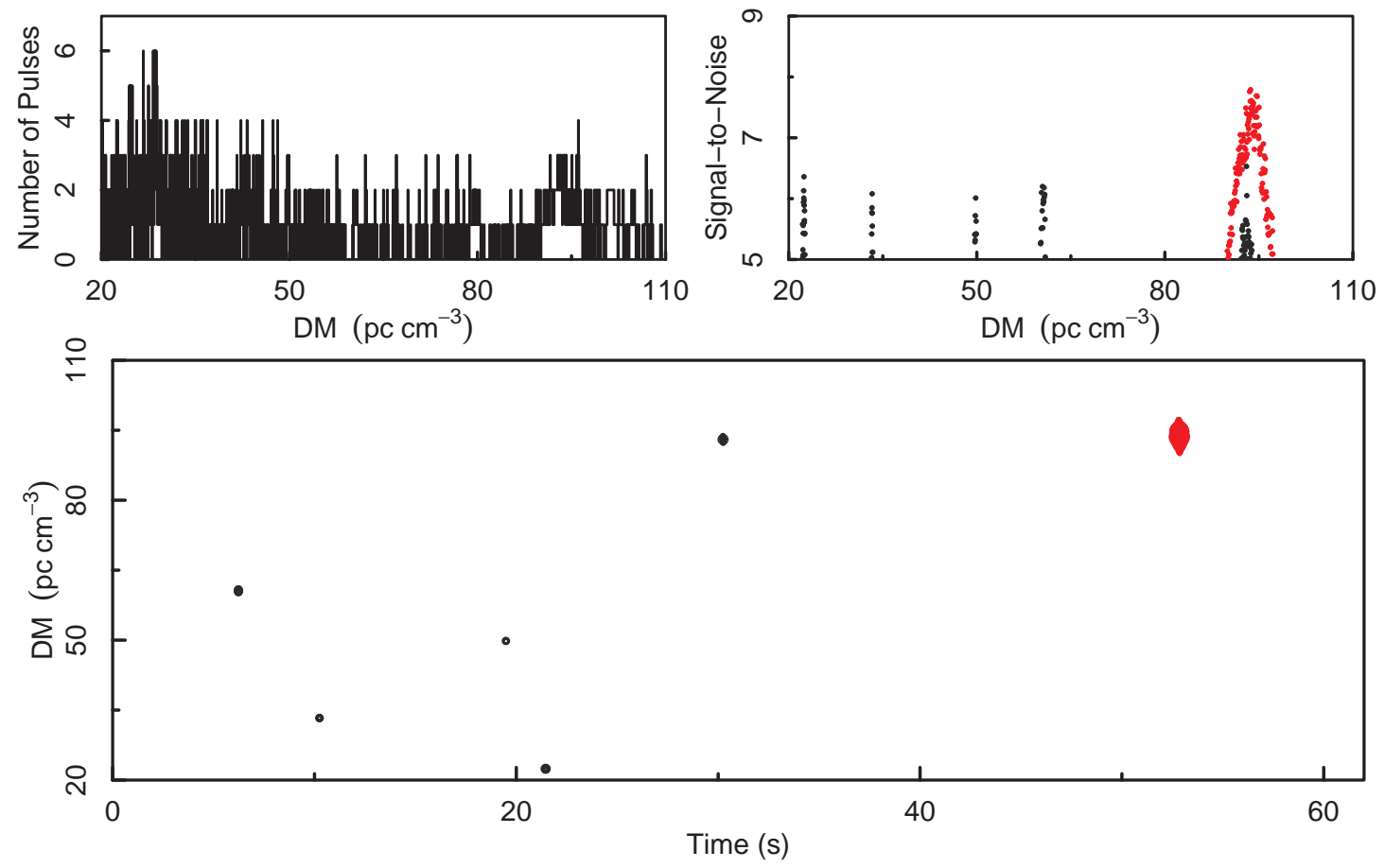

Figure 6.1: Single-pulse search diagnostic plot of the discovery observation of pulsar J0327+09. Only one relatively bright pulse candidate (plotted in red) with peak S/N of 7.79 is observed at $\mathrm{t} \sim 52.8 \mathrm{~s}$ in the beam. Compared with a standard single-pulse search diagnostic plot, the number of pulses versus $\mathrm{S} / \mathrm{N}$ subplot is omitted because it is not as informative as other subplots, especially for low $\mathrm{S} / \mathrm{N}$ pulsars. The comparison between the top and bottom two subplots shows the denosing effect of SPEGID. 
4bit_puppi_56971_Strip30_0809.0002.D034240+2757_0001 DM: 58.94 Period: NA S
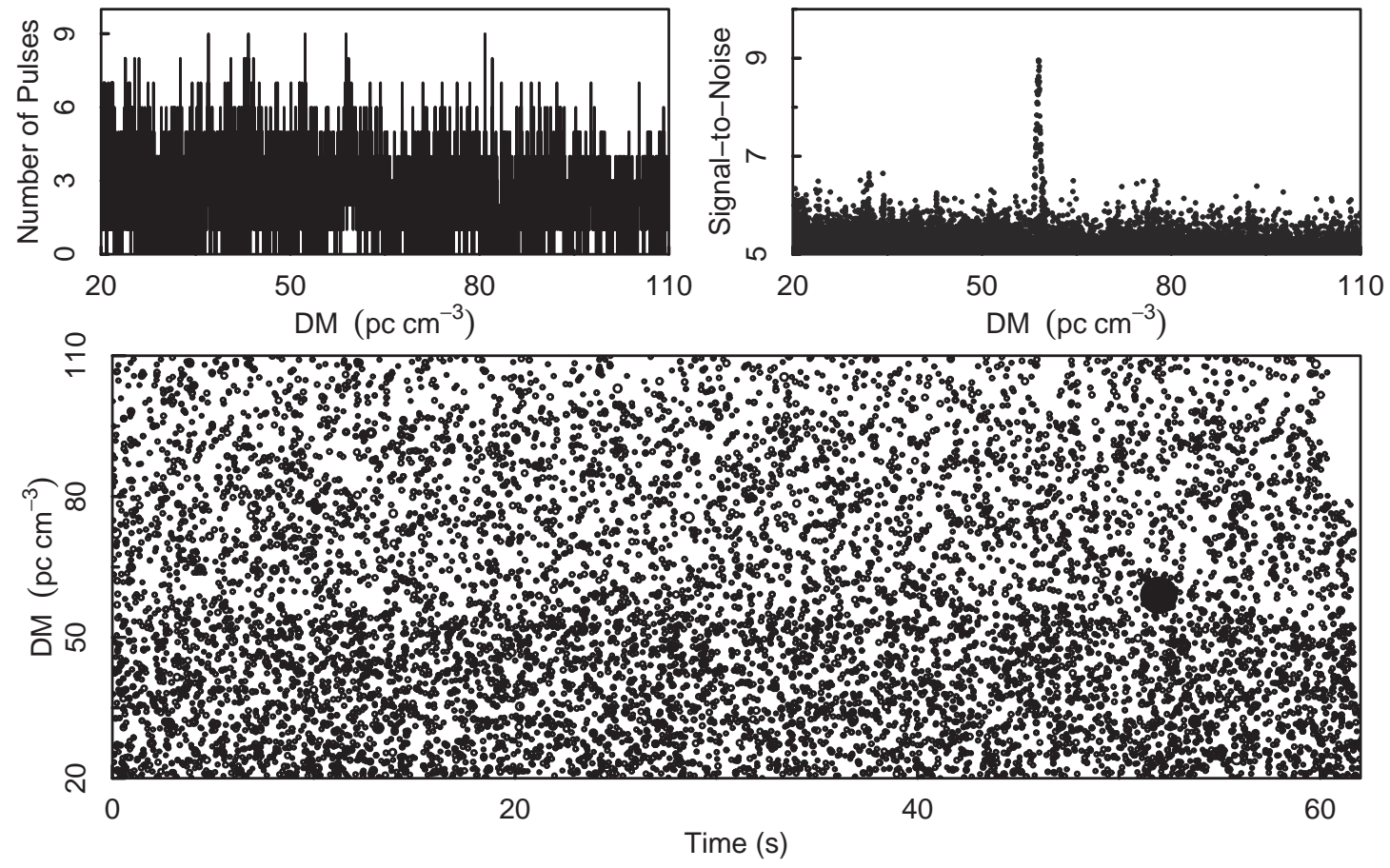

Pulse Candidates Identified by SPEGID
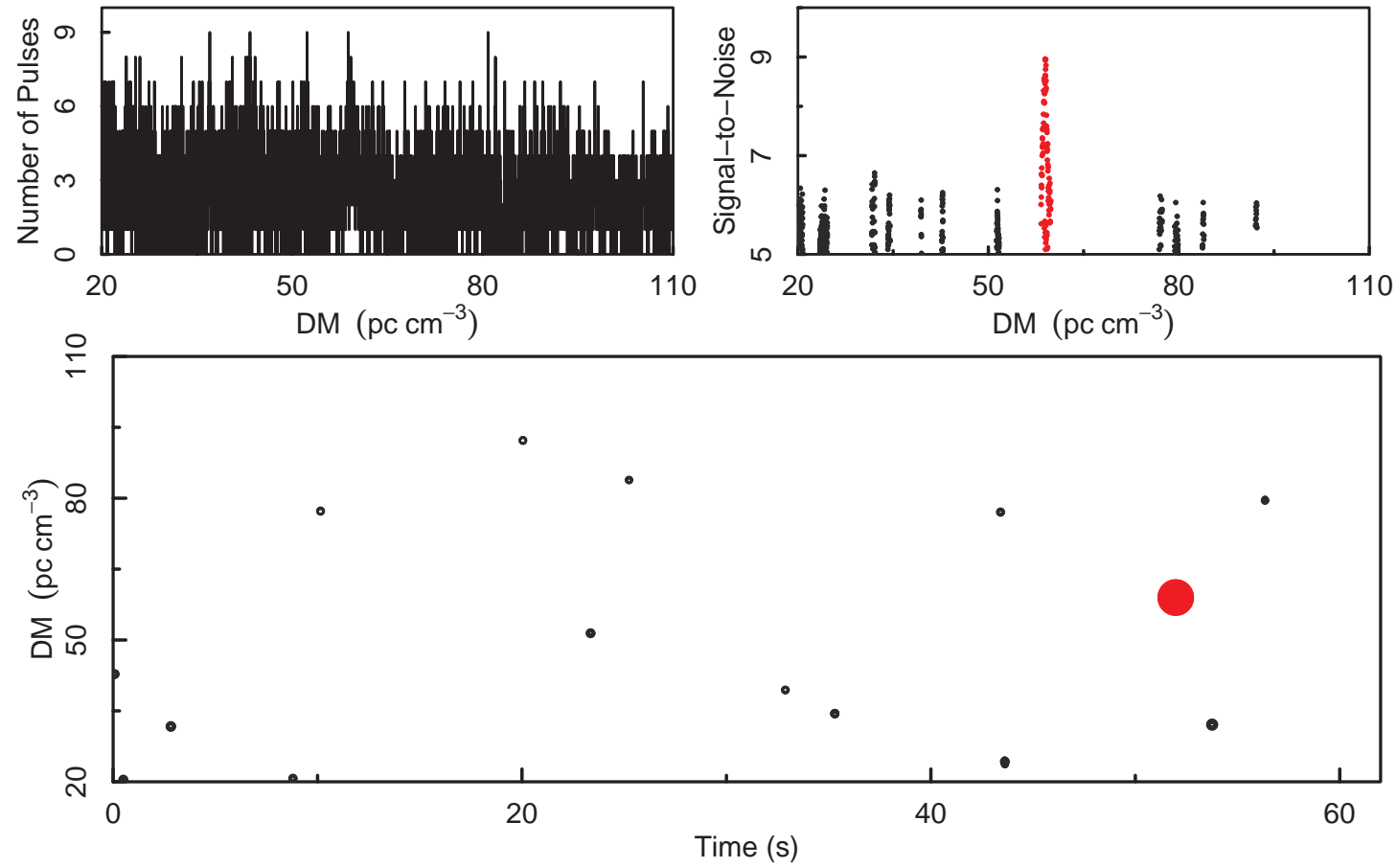

Figure 6.2: Single-pulse search diagnostic plot of the discovery observation of pulsar $\mathrm{J} 0342+27$. Only one relatively bright pulse (plotted in red) with peak $\mathrm{S} / \mathrm{N}$ of 8.96 is observed at $\mathrm{t} \sim 52.0 \mathrm{~s}$ in the beam. 
4bit_puppi_57732_Strip126_0262.0001.D153240+0042_0001 DM: 12.25 Period: NA s
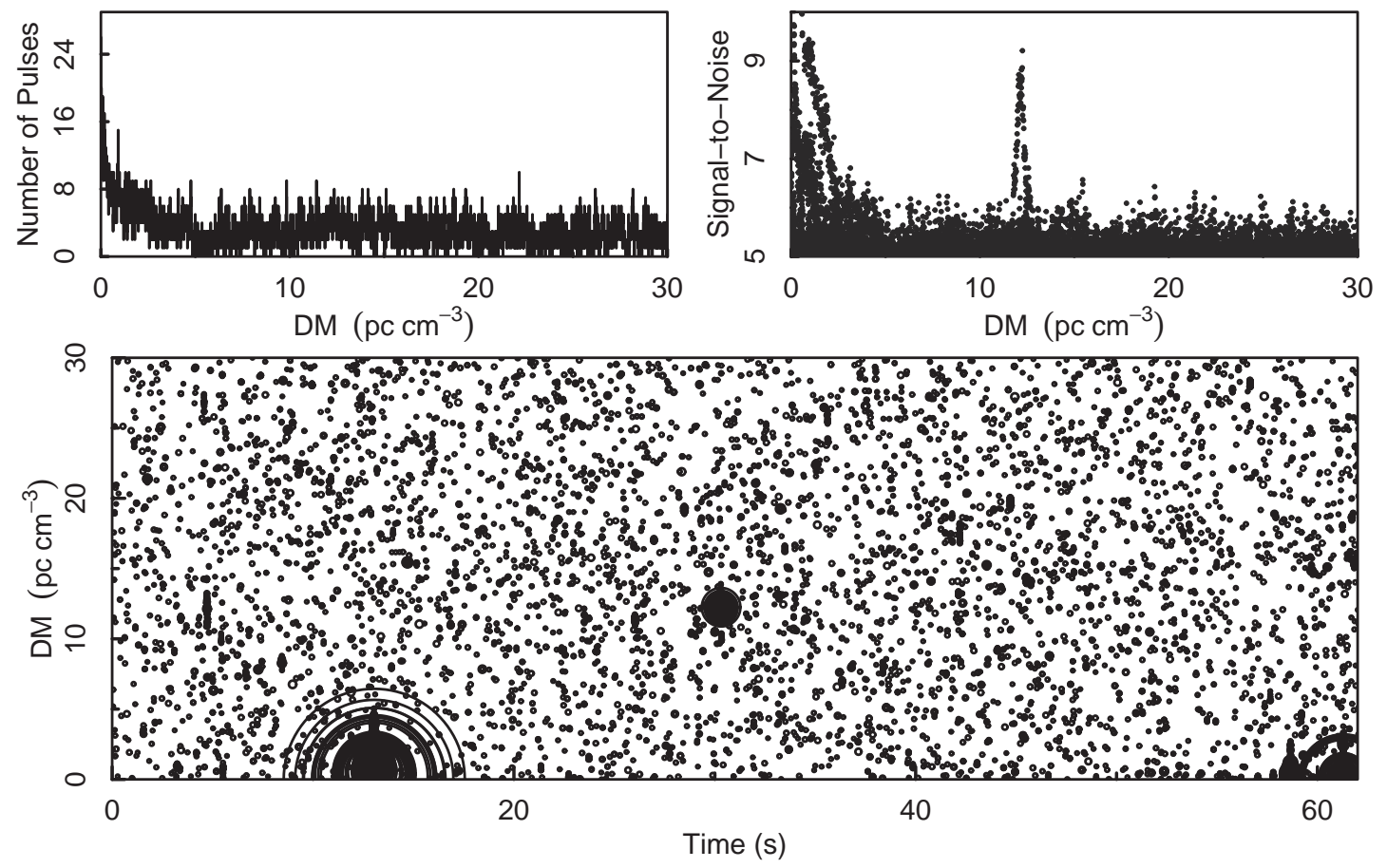

Pulse Candidates Identified by SPEGID
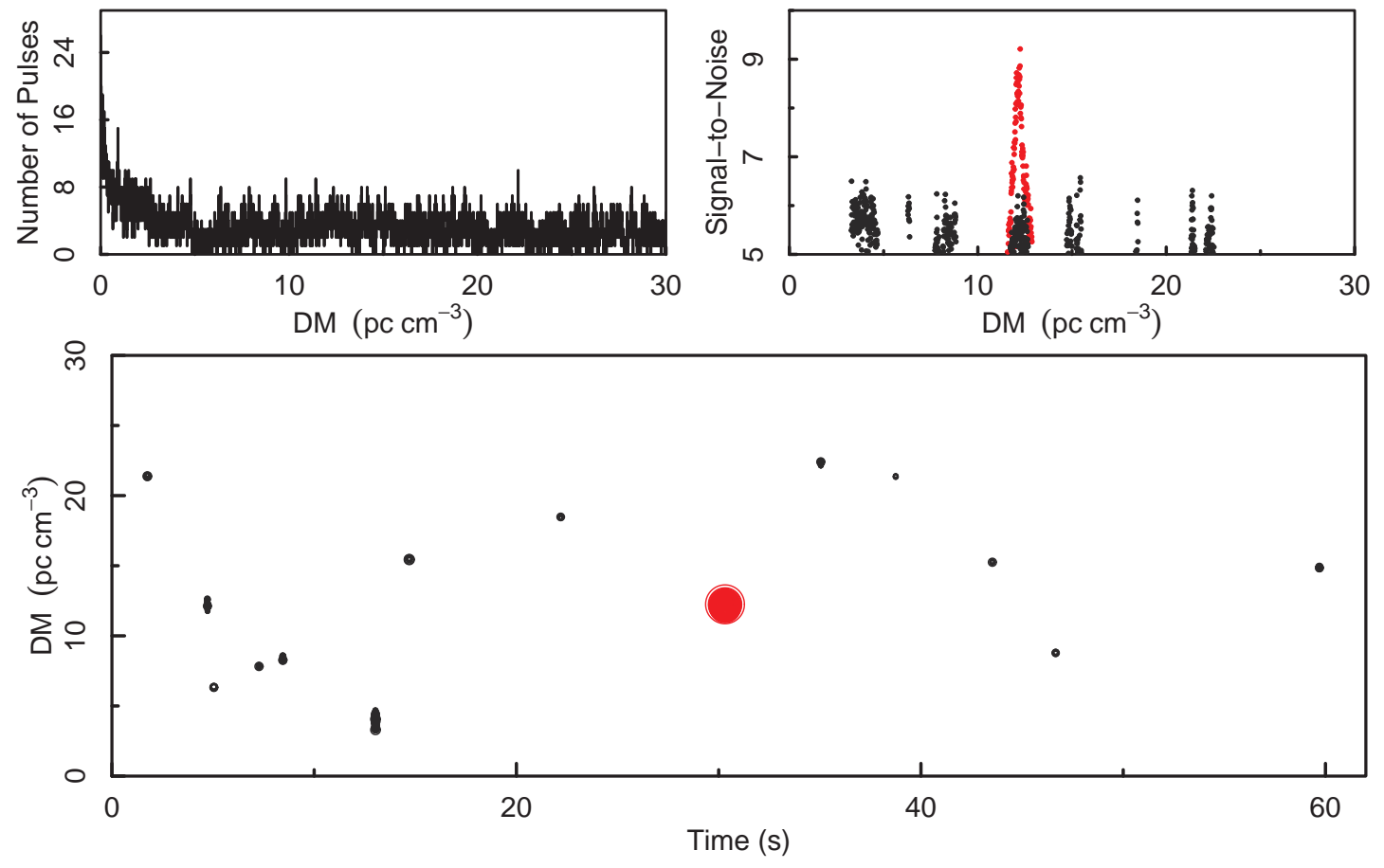

Figure 6.3: Single-pulse search diagnostic plot of the discovery observation of pulsar $\mathrm{J} 1532+00$. Only one relatively bright pulse candidate (plotted in red) with peak S/N of 9.21 is observed at $\mathrm{t} \sim 30.3 \mathrm{~s}$ in the beam. 
4bit_puppi_57145_Strip29_1185.0003.D220509+2742_0001 DM: 73.58 Period: $2.0206 \mathrm{~s}$
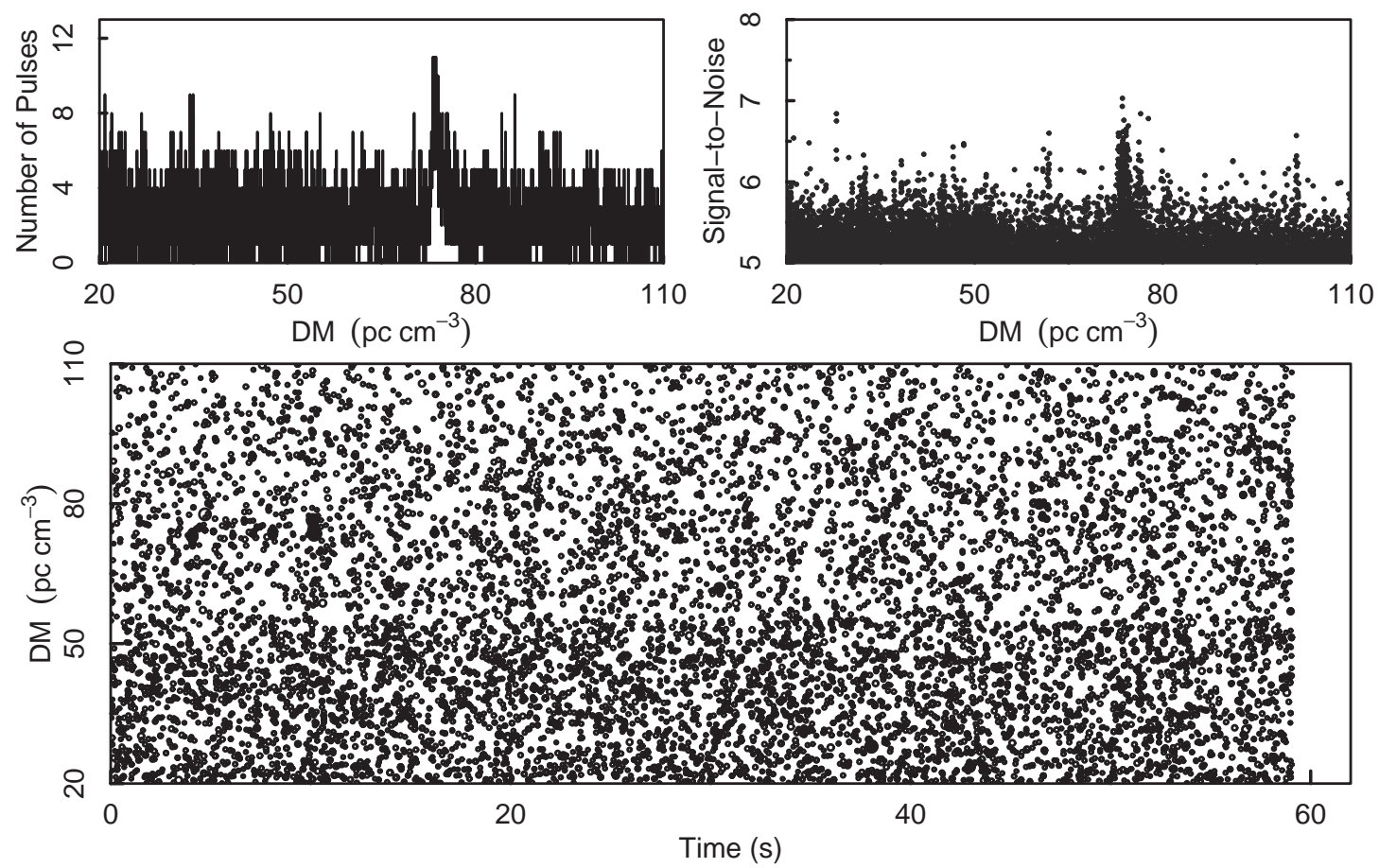

Pulse Candidates Identified by SPEGID
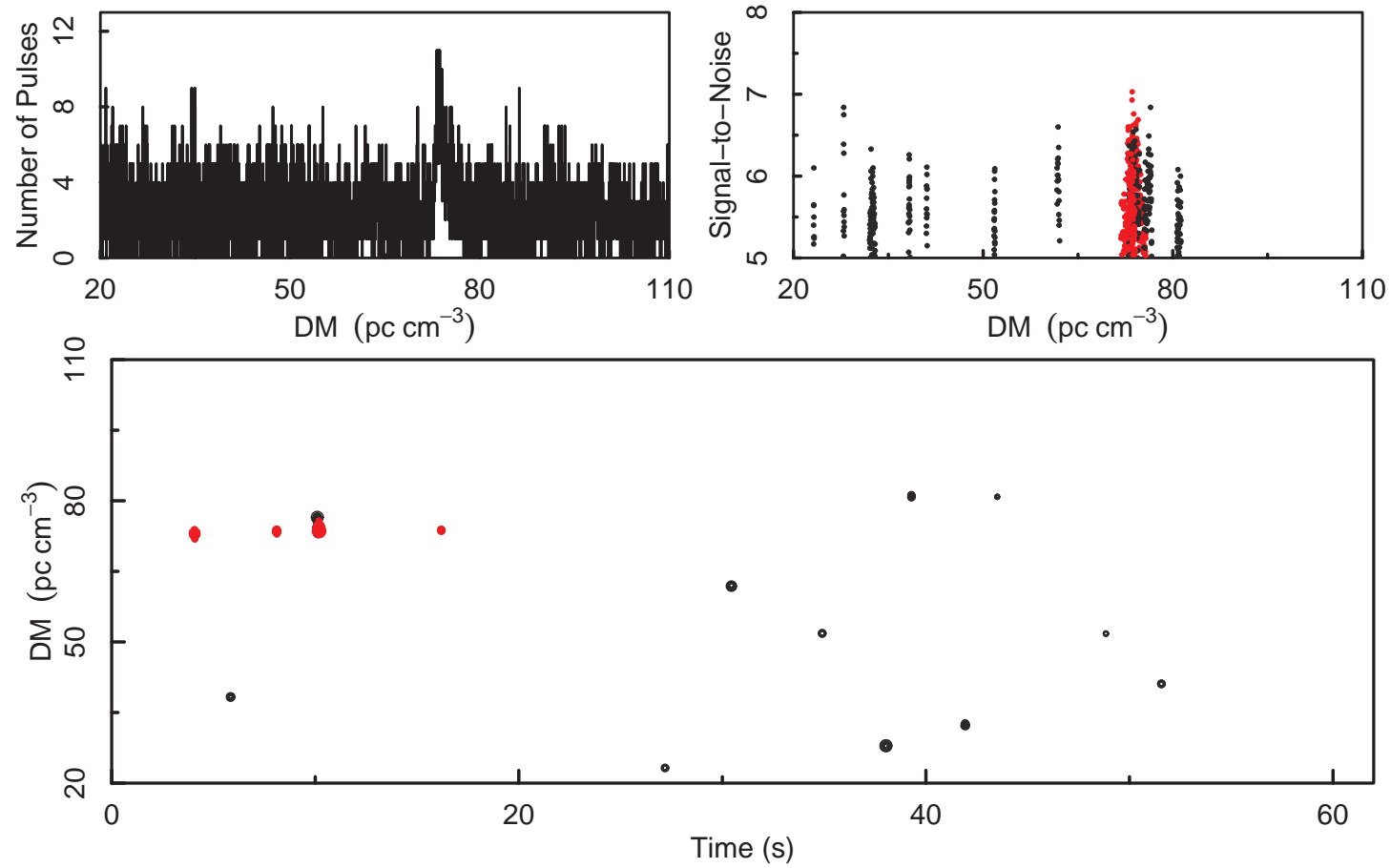

Figure 6.4: Single-pulse search diagnostic plot of the discovery observation of pulsar J2205+27. Four pulse candidates with underlying period of $2.0206 \mathrm{~s}$ were observed (plotted in red). The brightest candidate has peak $\mathrm{S} / \mathrm{N}$ of 7.03 is observed at $\mathrm{t} \sim 10.2$ $\mathrm{s}$ in the beam. Note not all four candidates may be classified as pulsar by our model. However, because an underlying periodicity is found among these four candidates, their probability of being astrophysical is significantly increased. 
4bit_puppi_58296_Strip16_0178.0001.D135440+2452_0001 DM: 20.07 Period: $0.7794 \mathrm{~s}$
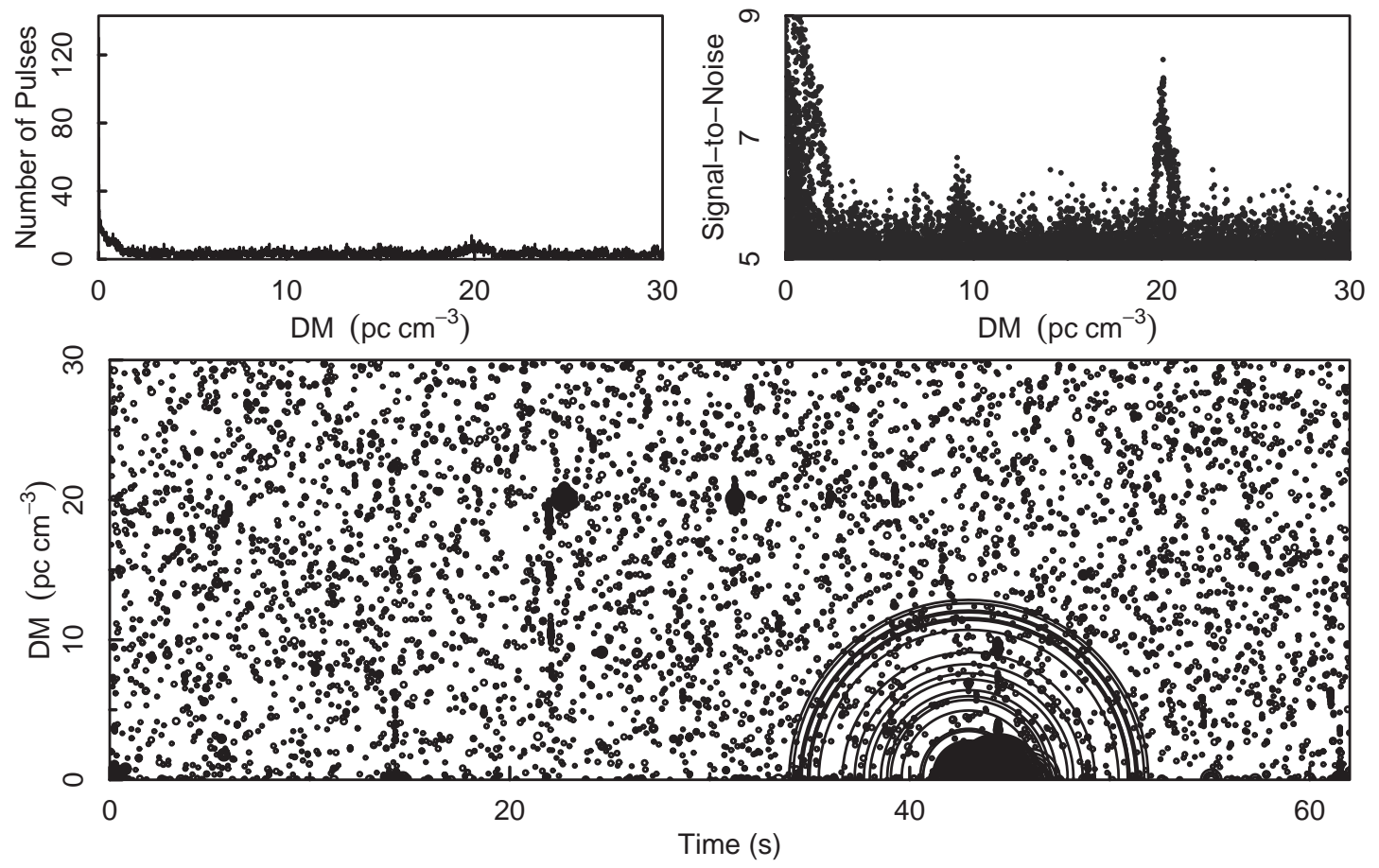

Pulse Candidates Identified by SPEGID
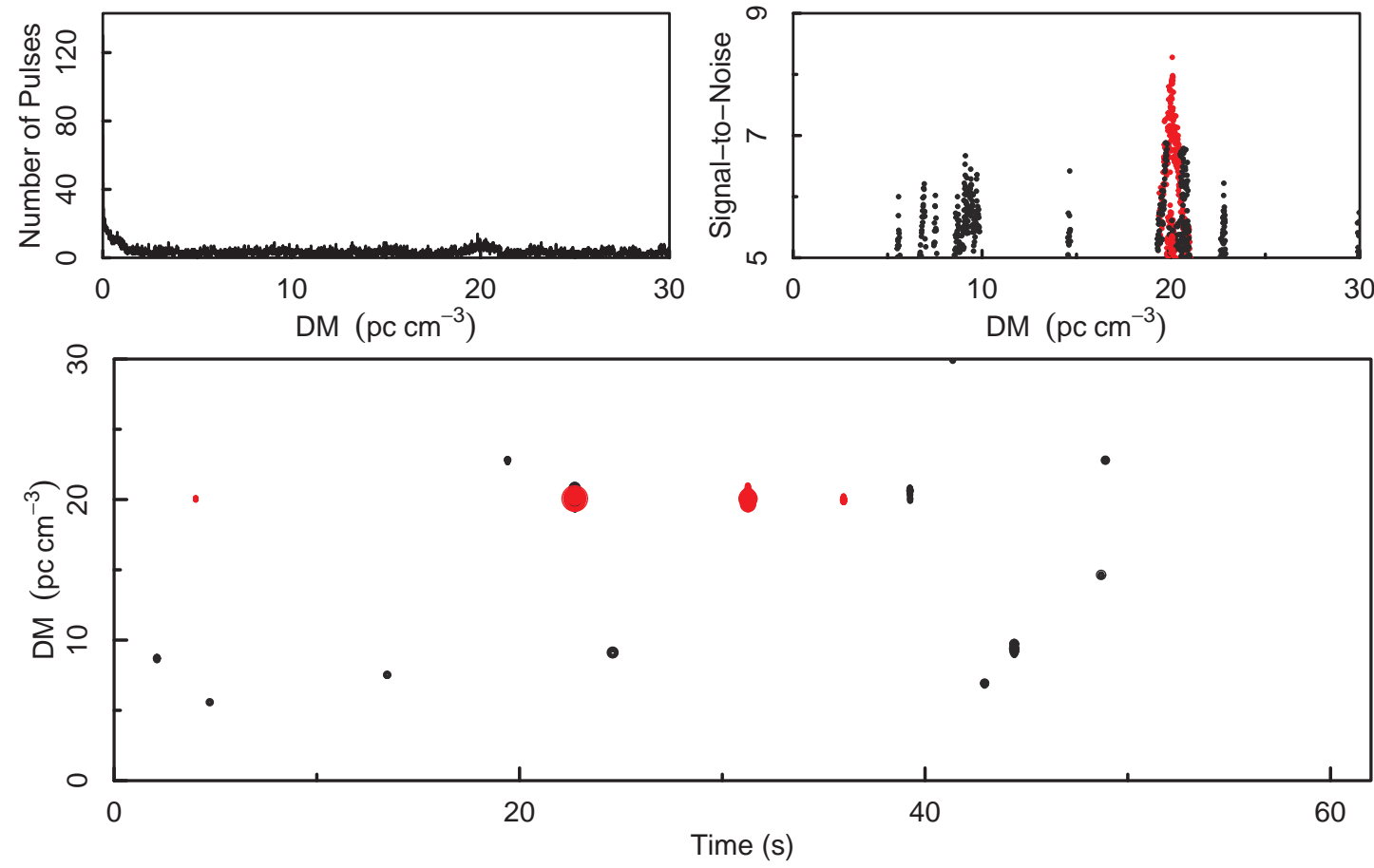

Figure 6.5: Single-pulse search diagnostic plot of the discovery observation of pulsar J1354+24. Four pulse candidates with underlying period of $0.7794 \mathrm{~s}$ were observed (plotted in red). The brightest pulse candidate has peak $\mathrm{S} / \mathrm{N}$ of 7.93 is observed at $\mathrm{t} \sim$ $22.7 \mathrm{~s}$ in the beam. 
4bit_puppi_56943_Strip35_0409.0001.D221123+1123_0001 DM: 32.67 Period: 0.55756 s
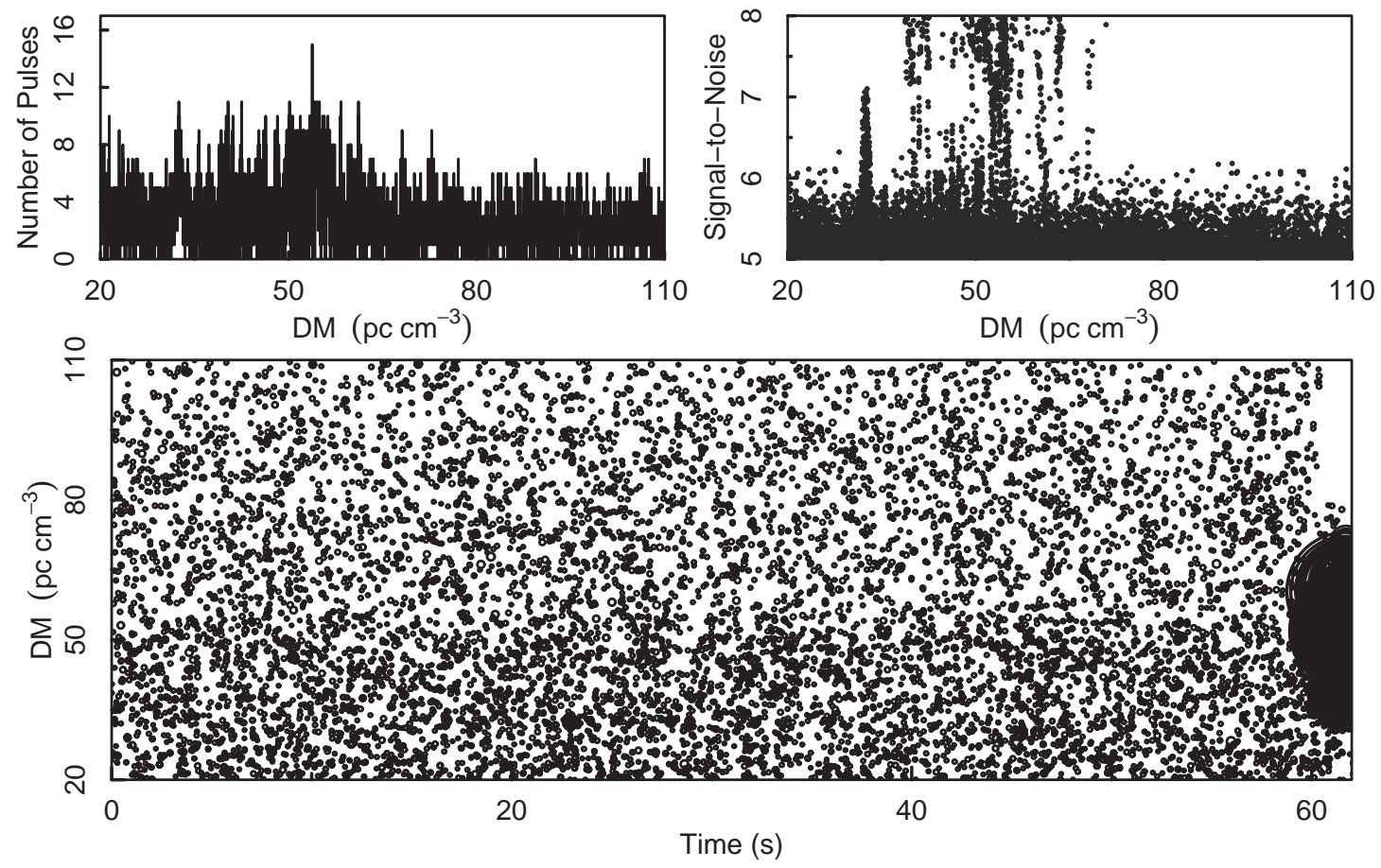

Pulse Candidates Identified by SPEGID
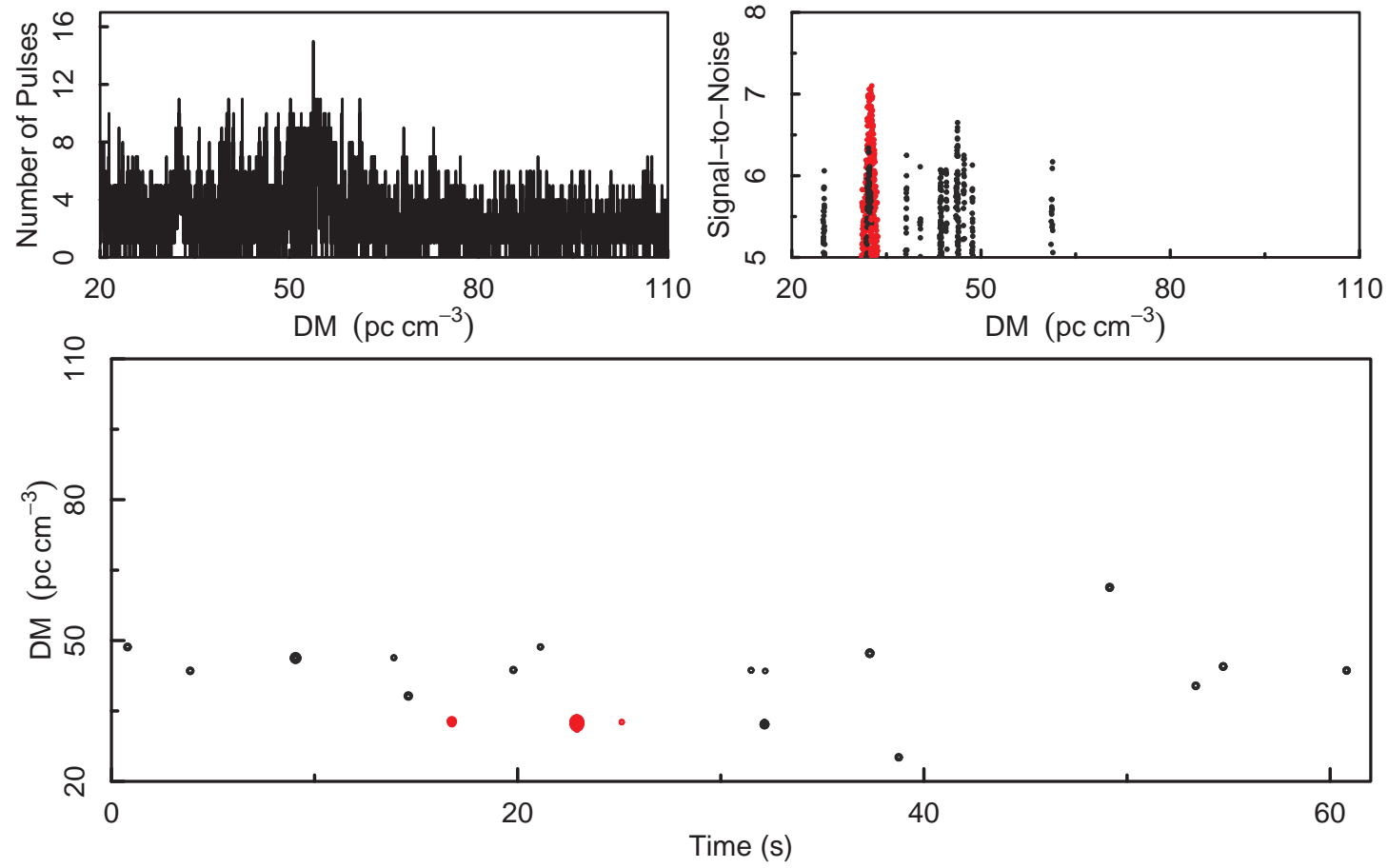

Figure 6.6: Single-pulse search diagnostic plot of the discovery observation of pulsar J2211+11. Three pulse candidates with underlying period of $0.55756 \mathrm{~s}$ were observed (plotted in red). The brightest pulse candidate has peak $\mathrm{S} / \mathrm{N}$ of 7.1 is observed at $\mathrm{t} \sim$ $25.1 \mathrm{~s}$ in the beam. 
4bit_puppi_58402_Strip35_0381.0001.D011012+1121_0001 DM: 14.86 Period: $0.43215 \mathrm{~s}$
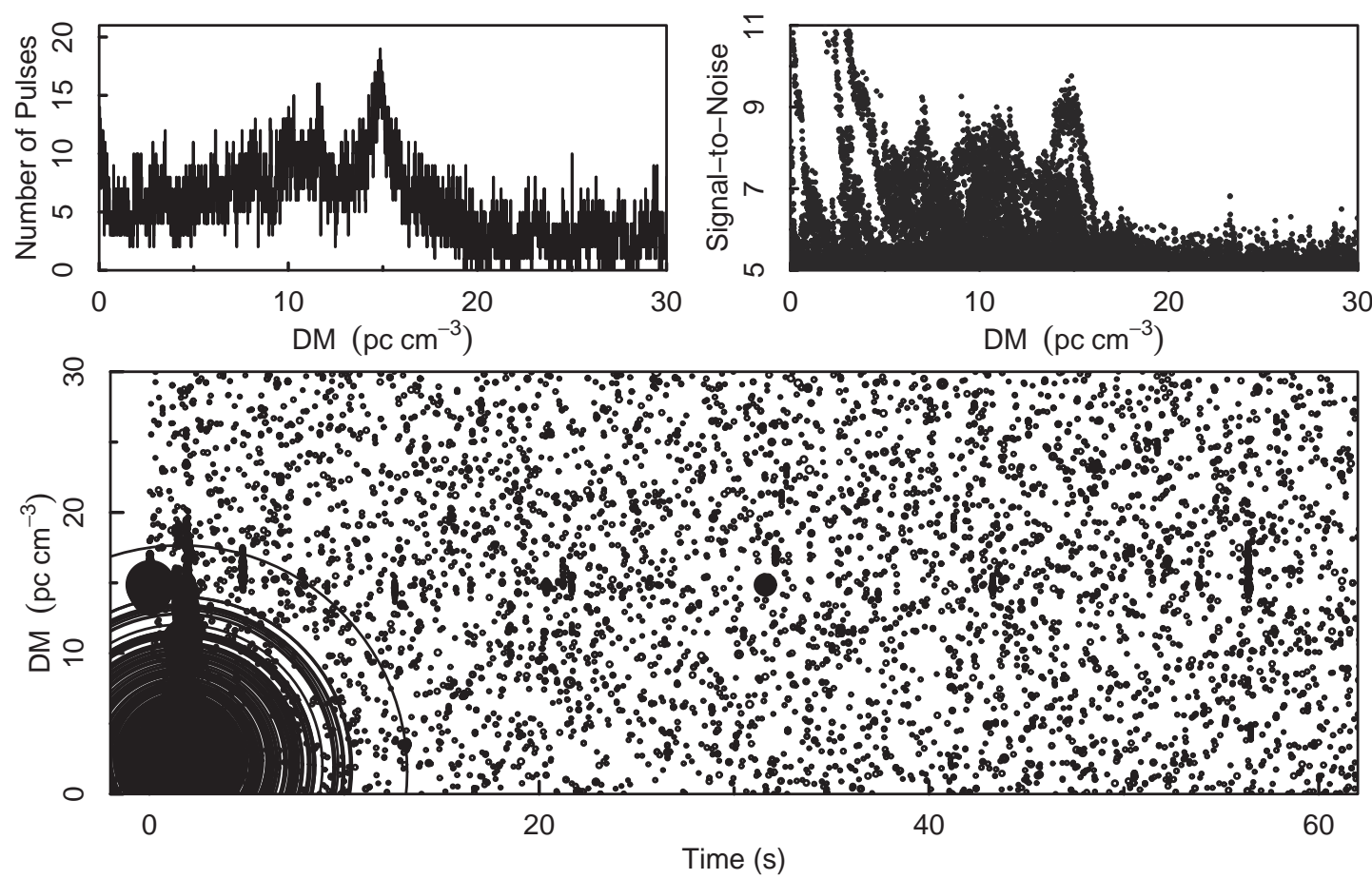

Pulse Candidates Identified by SPEGID
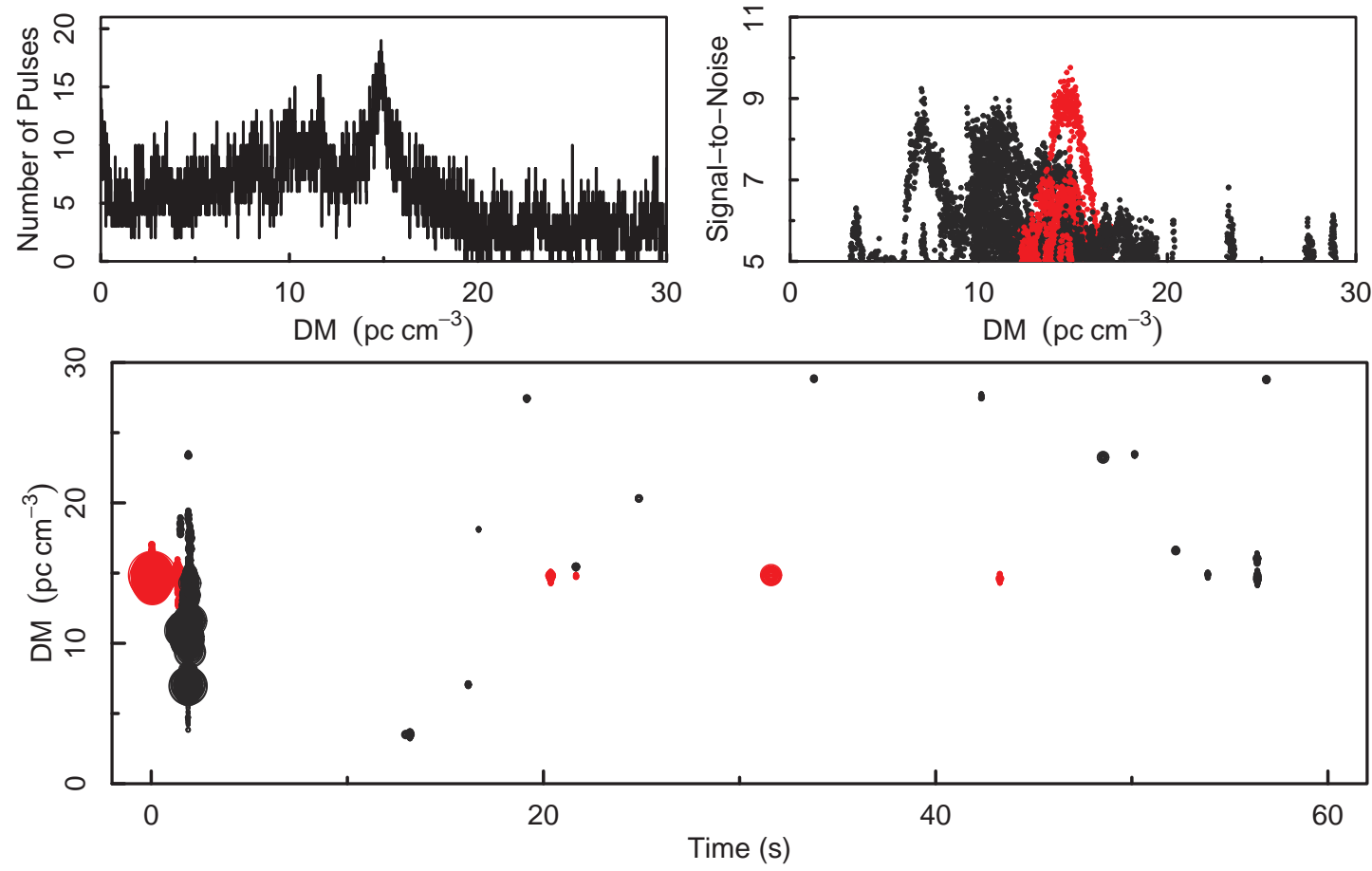

Figure 6.7: Single-pulse search diagnostic plot of the discovery observation of pulsar J0110+11. Eight pulses candidates (plotted in red) with underlying period of $0.43215 \mathrm{~s}$ were observed. The brightest pulse candidate has peak $\mathrm{S} / \mathrm{N}$ of 9.76 is observed at $\mathrm{t} \sim$ $0.05 \mathrm{~s}$ in the beam. It was missed by Clusterrank most likely because of the strong RFI contamination observed in the beam. The calculated period agrees with the periodicity found in the re-observation. 
4bit_puppi_58226_Strip958_0152.0001.D203043+0155_0001 DM: 50.82 Period: 1.6916 s
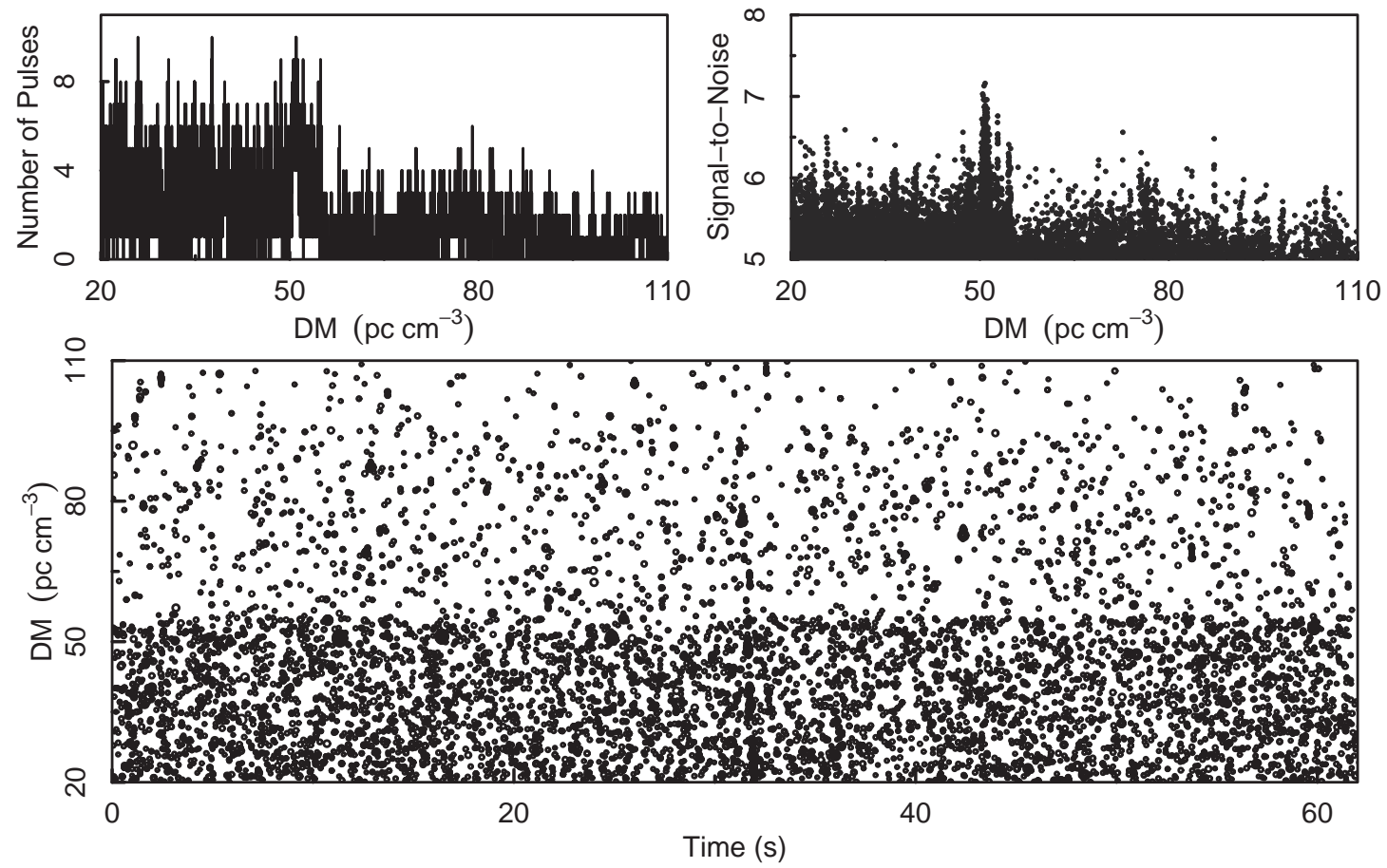

Pulse Candidates Identified by SPEGID
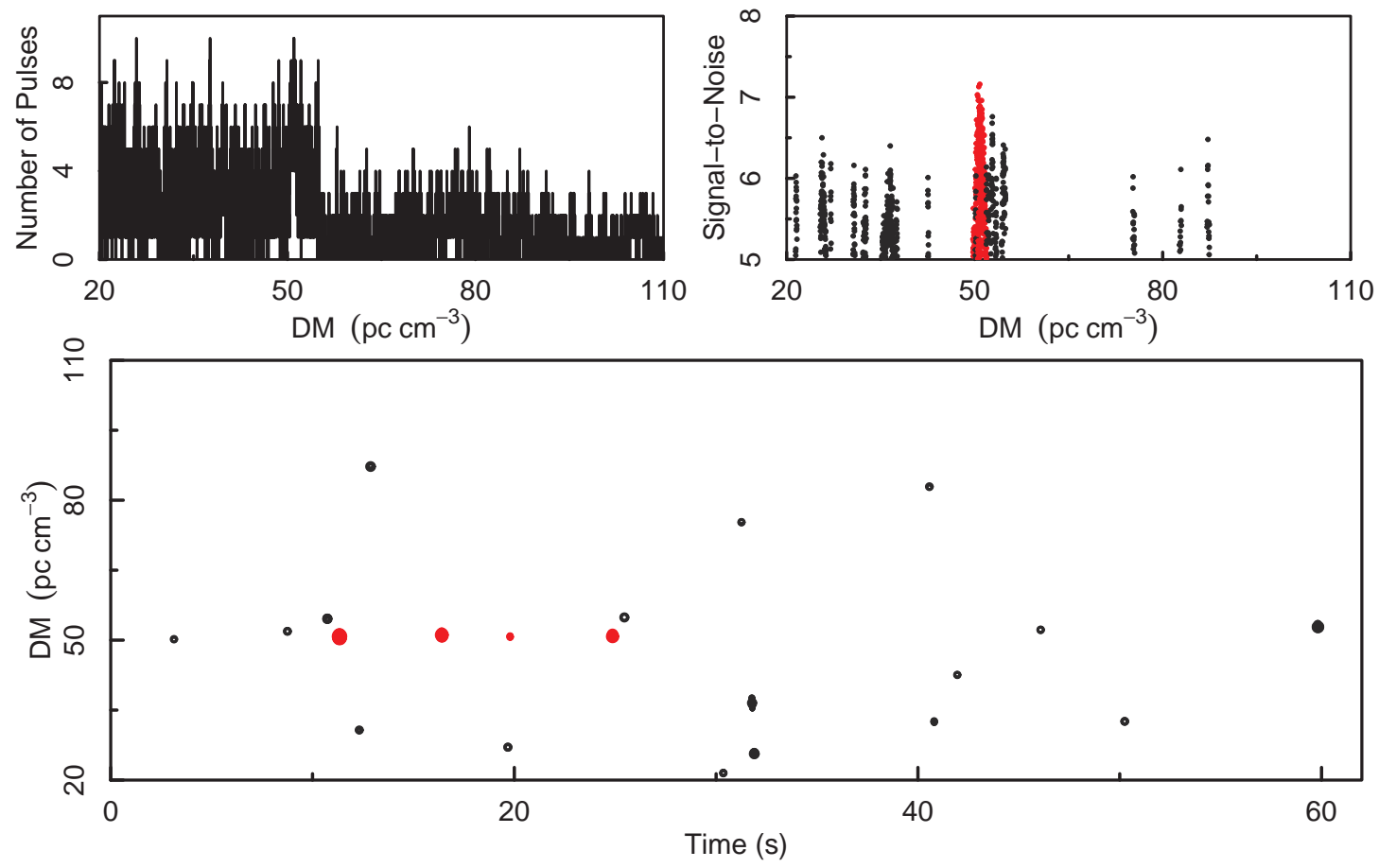

Figure 6.8: Single-pulse search diagnostic plot of the observation of pulsar J2027+02. Four pulse candidates with underlying period of $1.6916 \mathrm{~s}$ were observed (plotted in red). The brightest pulse candidate has peak $\mathrm{S} / \mathrm{N}$ of 7.16 is observed at $\mathrm{t} \sim 11.3 \mathrm{~s}$ in the beam. This pulsar was later discovered by FAST telescope in August 2019. 


\section{Chapter 7}

\section{Pulsar Classification Across Surveys}

In chapters 4 and 5, we investigated within-survey single pulse candidate selection, i.e., both the training data (on which the models are built) and the test data are from the same pulsar survey. Traditional machine learning assumes that the training data have the same (marginal and conditional) distribution as the test data. However, this is not necessarily true for different pulsar surveys because they often operate at different frequencies. If there are significant differences between radio data collected in different surveys, when a model that is trained on data from one survey (i.e., source survey) is used for single pulse classification in a different survey (i.e., target survey), its performance may degrade notably. Consequently, in order to classify pulsars with good performance, one course of action is to create a fully labeled benchmark data set for each pulsar survey, which is the current state-of-the-practice (e.g., [30, 79]). However, creating a labeled benchmark data set is a tedious and time-consuming process. Even more, at the early stage of a given survey, there may not be many detected pulsars that can be included into the training set. Without enough training data, the generalization error of supervised machine learning can be unsatisfactorily large.

In this chapter, we study how pulsar classification models perform when they are tested on data from another survey. We investigate two transfer learning techniques: trAdaBoost, an instance-based transfer learning method, and fine-tuning SPEGnet, a parameter-based transfer learning method ${ }^{1}$. The two benchmark data sets are GBTDrift and PALFA-extended as described in Chapter 5 . Note that since our two benchmark data

\footnotetext{
${ }^{1}$ The work presented in this chapter was published in [80].
} 
sets are very large (in terms of the number of SPEGs), feature-based transfer learning techniques, which are based on matrix computations, can easily result in out-of-memory error and therefore are not practical [71].

\subsection{Cross-survey Prediction without Transfer Learning}

Our results in Chapter 5 have shown that: (i) to achieve satisfactory prediction results, at least 20 representative pulsars should be included in the benchmark data set; (ii) more data usually improve the prediction. Because creating a fully labeled benchmark data set is a time-consuming task and it may not even be possible at the beginning of a new survey, it is natural to consider using labeled benchmark data from one survey to classify the unlabeled data collected in another survey.

In most machine learning problems, however, the classification performance of a model is affected when there are significant differences between the distributions of the training and testing data. In this section, we explore how well models trained on benchmark data from one survey perform when tested on benchmark data from another survey.

\subsubsection{Classifying GBTDrift Using Models Trained on PALFA-extended}

Although some degree of performance degradation is expected, the results in Table 7.1 show that models trained on PALFA-extended and tested on GBTDrift have much worse performance (measured by recall and $\mathrm{F}_{4}$ ) than the models trained and tested on GBTDrift (i.e., within survey prediction). In particular, the performance of RandomForest degrades the most (0.412 in recall and 0.401 in $\mathrm{F}_{4}$ measure), and the performance of AdaBoost degrades the least (0.244 in recall and 0.239 in $\mathrm{F}_{4}$ measure).

Table 7.1: Comparison of model performance between within-survey (trained and tested on GBTDrift, also shown in Table 5.3) and cross-survey (trained on PALFA-extended, tested on GBTDrift) predictions.

\begin{tabular}{|l|l|c|c|c|}
\hline Type & Metric & RandomForest & AdaBoost & SPEGnet \\
\hline \multirow{2}{*}{ Within } & Recall & $0.906 \pm 0.001$ & $0.985 \pm 0.001$ & $0.974 \pm 0.002$ \\
\cline { 2 - 5 } & $F_{4}$ & $0.902 \pm 0.001$ & $0.942 \pm 0.001$ & $0.934 \pm 0.001$ \\
\hline \multirow{2}{*}{ Across } & Recall & $0.494 \pm 0.002$ & $0.741 \pm 0.004$ & $0.621 \pm 0.004$ \\
\cline { 2 - 5 } & $F_{4}$ & $0.501 \pm 0.002$ & $0.703 \pm 0.004$ & $0.604 \pm 0.005$ \\
\hline
\end{tabular}




\subsubsection{Classifying PALFA-extended Using Models Trained on GBTDrift}

Similarly, when the models are trained on GBTDrift (i.e., source) and tested on PALFA-extended (i.e., target), their performance (measured by recall and $\mathrm{F}_{4}$ ) degrades significantly, as shown in Table 7.2. Specifically, RandomForest shows the highest degradation in recall (0.127), and the SPEGnt shows the highest degradation in terms of $\mathrm{F}_{4}$ measure $(0.253)$. It is worth mentioning that for cross-survey prediction, the high recall and low $\mathrm{F}_{4}$ measure of SPEGnet and AdaBoost indicates very high FPR, effectively rendering these models useless. Compared with when the PALFA-extended was used as the source and GBTDrift as the target data set, the degradation of RandomForest is relatively small.

Table 7.2: Comparison of model performance between within-survey (trained and tested on PALFA-extended, also shown in Table 5.4) and cross-survey (trained on GBTDrift, tested on PALFA-extended) predictions.

\begin{tabular}{|l|l|c|c|c|}
\hline Type & Metric & RandomForest & AdaBoost & SPEGnet \\
\hline \multirow{2}{*}{ Within } & Recall & $0.916 \pm 0.001$ & $0.945 \pm 0.012$ & $0.948 \pm 0.011$ \\
\cline { 2 - 5 } & $F_{4}$ & $0.890 \pm 0.001$ & $0.867 \pm 0.001$ & $0.877 \pm 0.001$ \\
\hline \multirow{2}{*}{ Across } & Recall & $0.789 \pm 0.009$ & $0.821 \pm 0.023$ & $0.909 \pm 0.010$ \\
\cline { 2 - 5 } & $F_{4}$ & $0.667 \pm 0.008$ & $0.648 \pm 0.019$ & $0.624 \pm 0.010$ \\
\hline
\end{tabular}

\subsection{Training on a Combined Data Set without Transfer Learning}

Although our results showed that models trained on benchmark data set from one survey performed poorly when they were tested on those from the other, they still performed much better than random guessing. This is because the astrophysical pulses share some common characteristics even when from different surveys [19]. Therefore, it follows that data from one survey contain information that can be used to improve the classification on another survey. In this section we present the results of training the models on a combined data set, but without transfer learning. Specifically, we experiment with combined data sets that contain the whole source data set and a limited amount of data available from the target data set. For example, when GBTDrift is treated as the target data set, we would include all 100 pulsars from PALFA-extended 
into the training data. These combined with pulsars from GBTDrift would increase the number of training pulsars from 5, 10 and 20 to 105, 110 and 120, respectively.

\subsubsection{Classifying GBTDrift Using Models Trained on a Combined Data Set}

The results presented in Figure 7.1 (a) show combining the PALFA-extended benchmark data set (source) with the limited amount of GBTDrift training data (with up to 20 pulsars) (target) has little impact on the $F_{4}$ measure of the RandomForest models, but significantly improves the $F_{4}$ measure of the AdaBoost models (Figure 7.1(b)). On the other hand, SPEGnet models trained on the combined data set have worse performace than when training only on the limited amount of target data (see Figure 7.1(c)). This is because the majority of the training data still consist of SPEGs from PALFA-extended; without transfer learning, the trained models would be similar to models trained only on PALFA-extended and therefore result in large generalization error when they are tested on GBTDrift.

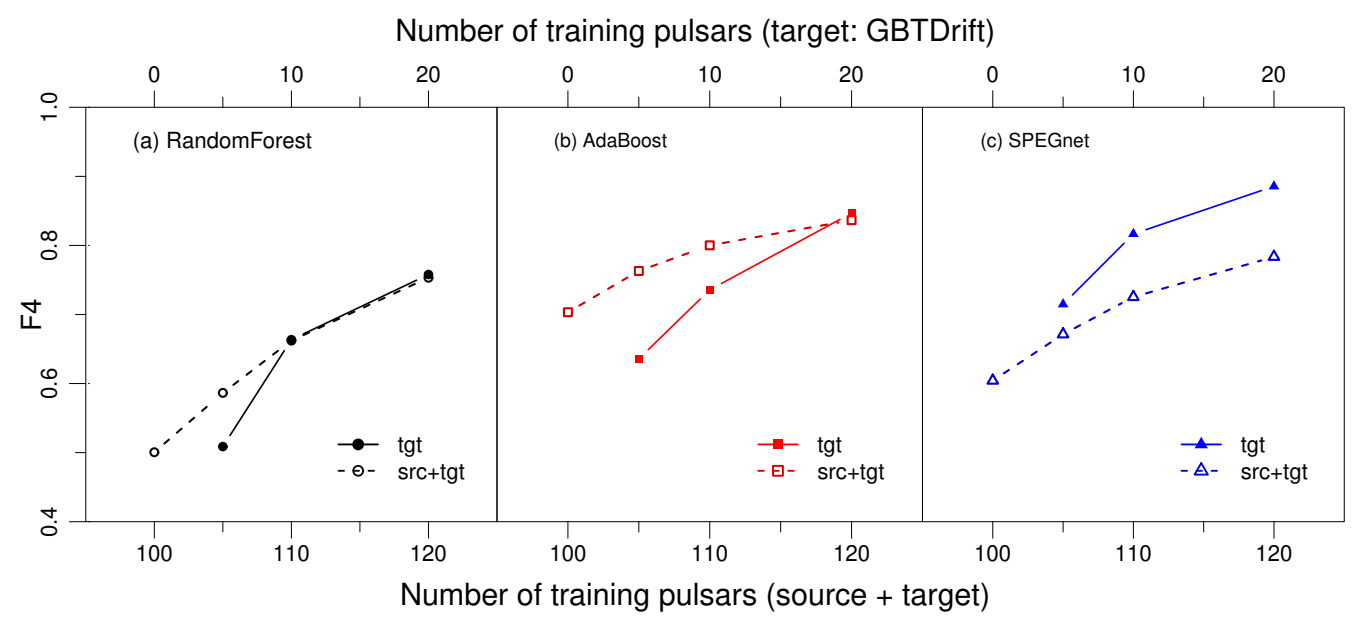

Figure 7.1: $F_{4}$ measure between models trained only on the target GBTDrift data and those trained on the combined source and target training data (i.e., the whole PALFA benchmark data set and up to 20 pulsars from the GBTDrift data). Note that one pulsar corresponds to tens, even hundreds, of training SPEGs. 


\subsubsection{Classifying PALFA-extended Using Models Trained on a Com- bined Data Set}

The results presented in Figure 7.2 show that ombining the GBTDrift benchmark data set (source) with the limited amount of PALFA-extended training data (with up to 20 pulsars) (target) improves the $F_{4}$ measure of the RandomForest and AdaBoost models (Figure 7.2 (a) and (b)), but does not improve the $F_{4}$ measure of the SPEGnet model.

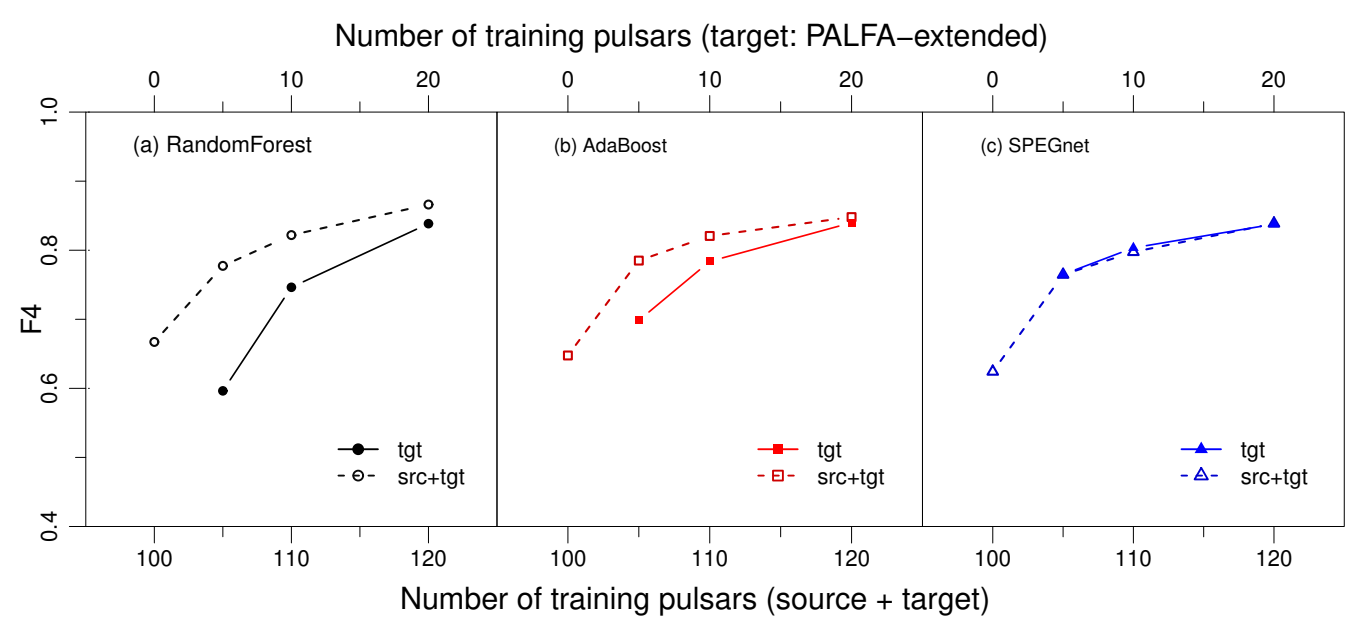

Figure 7.2: Comparison of $F_{4}$ measure between models trained on the target PALFA data and those trained on the combined source and target training data (i.e., the whole GBTDrift benchmark data set and up to 20 pulsars from the PALFA data). Note that one pulsar corresponds to tens, even hundreds of training SPEGs.

\subsection{Cross-survey Results with Transfer Learning}

Our results have shown that if measured by $\mathrm{F}_{4}$ measure, simply combining labeled data from another survey does not always improve the classification. In order to achieve consistent improvement, transfer learning is necessary. In this section, we use the models that performed the best on the combined training data set (i.e., AdaBoost for GBTDrift and RandomForest for PALFA-extended) and compare them with models generated by two different transfer learning techniques, namely trAdaBoost and fine-tuning of SPEGnet. SPEGnet models trained on target data only are also included to show the effect of fine-tuning more clearly. 


\subsection{1 trAdaBoost}

trAdaBoost is a classic instance-based transfer learning algorithm [20]. It assumes that the source and target domain share the same number of features and the same task, but have different distributions. Such distribution differences can lead to performance degradation. However, a part of the data from the source domain may be similar to data from the target domain, and therefore can be useful for learning in the target domain. trAdaBoost uses the AdaBoost strategy to assign higher weights to these similar data and lower weights to the rest of data in the source domain. This way, trAdaBoost combines useful data in the source domain with data in the target domain (where labeled data may be limited) without affecting its distribution. In our experiments, we used RandomForest as the base learner for trAdaBoost. As for class imbalance treatment, we found that trAdaBoost worked the best when the source data set was treated using RUS and the target data set was treated with SMOTE.

\subsubsection{Fine-tuning SPEGnet}

Training deep neural networks with random initialization requires a large amount of data and training time. Fortunately, fine-tuning pre-trained neural networks solves this problem. This is because concepts learned by the lower level layers are observed to be generic features that should be transferable to many tasks, while concepts learned by later layers become progressively more specific to the details of the classes that are contained in the original data set [111]. Therefore, we investigate if the best transferability of features is achieved by re-training the whole pre-trained SPEGnet model using the target training data, or if it is sufficient to just fine-tune one, two, or three higher-level layers. We found that the best way to fine-tune the pre-trained SPEGnet is to: (1) freeze the first three hidden layers and only fine-tune the last hidden layer for GBTDrift; (2) freeze the first five hidden layers and fine-tune the last two hidden layers for PALFA-extended. In addition, we found that the best class imbalance treatment for fine-tuning was to apply SMOTE to both the source and target data sets.

\subsubsection{Hyperparameter Tuning}

Like SPEG classification within a survey, for cross-survey prediction using transfer learning, the hyperparameters were tuned using nested 6 -fold cross-validation. The outer loop ran over all 6 folds with one fold reserved for testing, and the inner loop performed a standard 5-fold cross-validation in order to select the best model hyperparameters [67]. 


\subsubsection{Classifying GBTDrift Using Transfer Learning}

The classification results of GBTDrift by the transfer learning (trAdaBoost and fine-tuning SPEGnet) produced models are shown in Figure 7.3, with AdaBoost models trained on the combined training data set without transfer learning and SPEGnet trained on target data only used as the baseline models.

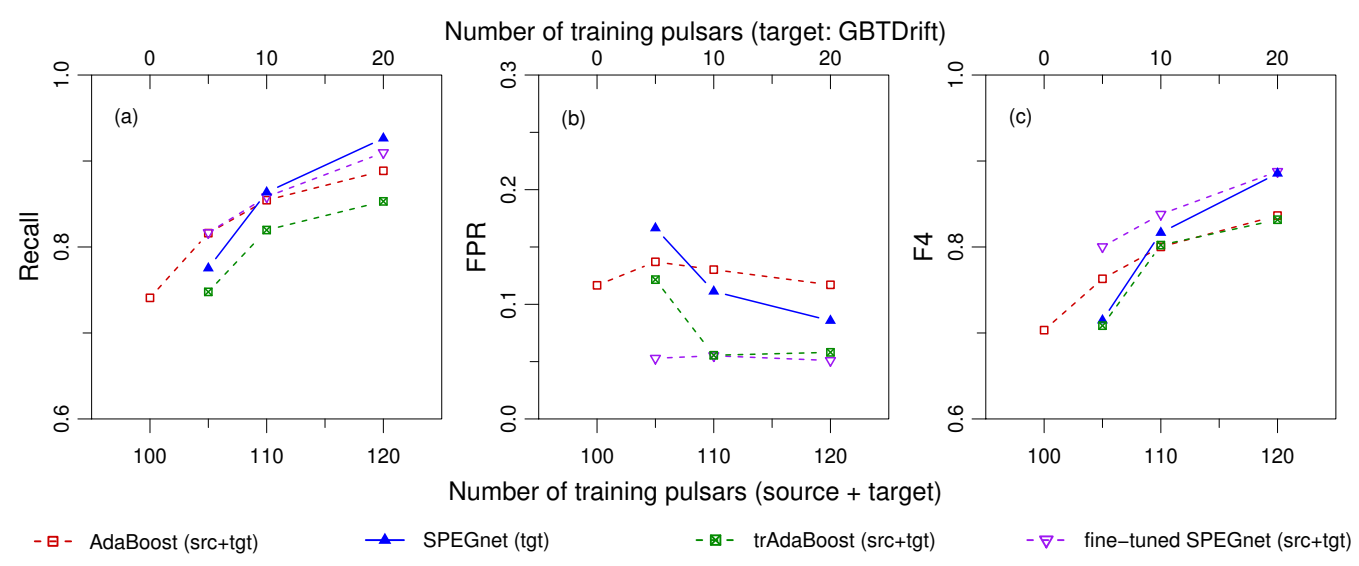

Figure 7.3: Comparison of transfer learning models (i.e., trAdaBoost and fine-tuning SPEGnet) with AdaBoost (trained on the combined data set without transfer learning) and SPEGnet models (trained on limited target data without transfer learning), when GBTDrift was used as target and PALFA-extended as source. Note that one pulsar corresponds to tens, even hundreds of training SPEGs.

Based on these results, the following observations are made:

- When the number of training pulsars from the target data is very limited $(\leq 10$ pulsars), fine-tuning SPEGnet and training AdaBoost on the combined data set without transfer learning have the highest recall. For more that 10 pulsars in the training data, SPEGnet model trained on target data only has the highest recall. trAdaBoost consistently results in the lowest recall.

- Both transfer learning techniques decrease the FPR (see Figure 7.3(b)). In contrast, AdaBoost trained on combined data set without transfer learning has the highest FPR.

- If the performance is measured by $\mathrm{F}_{4}$ measure, as shown in Figure 7.3(c), finetuned SPEGnet models consistently outperforms the other models. This indicates the related information from the source data set was extracted by SPEGnet and 
retained by freezing the lower layers. On the other hand, the improvement of finetuning SPEGnet becomes less significant as more pulsars from the target data set are included into the training data. Once there are 20 labeled pulsars from the target data set available, the difference of $\mathrm{F}_{4}$ measure between fine-tuned SPEGnet and SPEGnet becomes negligible, that is, transfer learning becomes unnecessary.

\subsubsection{Classifying PALFA-extended Using Transfer Learning}

The classification results of PALFA-extended by the transfer learning (trAdaBoost and fine-tuning SPEGnet) produced models are shown in Figure 7.4, with RandomForest models trained on the combined training data set without transfer learning and SPEGnet trained on target data only used as the baseline models.

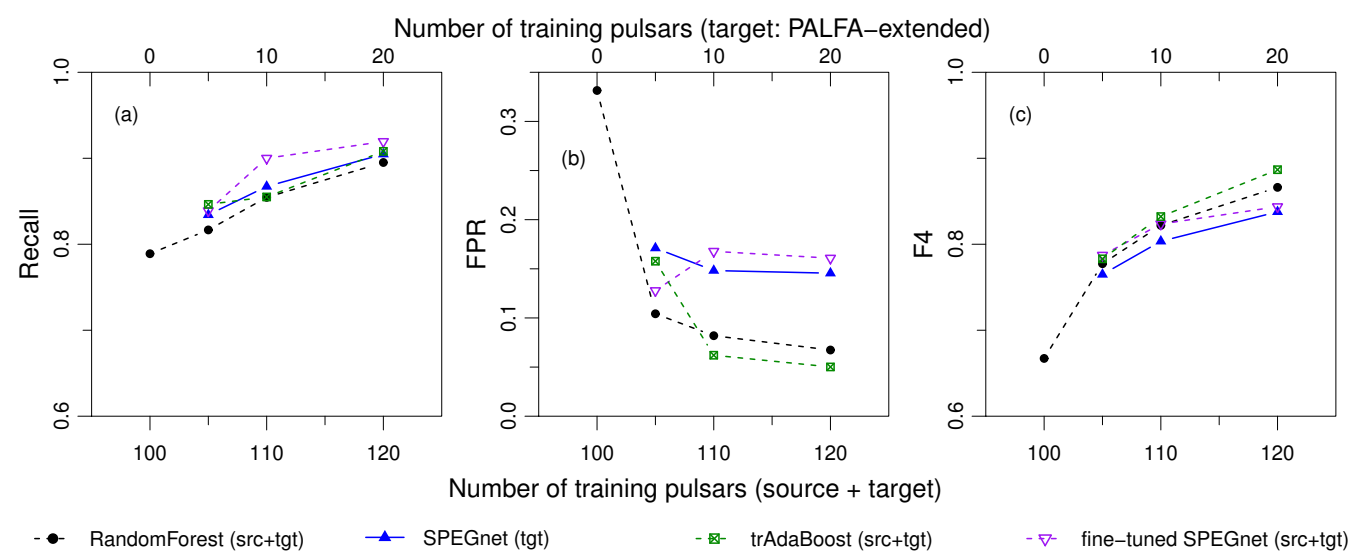

Figure 7.4: Comparison of transfer learning models trAdaBoost and fine-tuning SPEGnet with RandomForest (trained on the combined data set without transfer learning) and SPEGnet models (trained on limited target data without transfer learning), when PALFA-extended was used as target and GBTDrift as source. Note that one pulsar corresponds to tens, even hundreds of training SPEGs.

Based on these results, we find:

- Regardless of the size of training data, fine-tuned SPEGnet models constantly have the highest recall, followed by the SPEGnet models without transfer learning.

- However, fine-tuned SPEGnet models and SPEGnet models without transfer learning usually have the highest FPR as well. When there are at least 10 training pulsars from the target PALFA-extended data set, trAdaBoost has the lowest FPR. 
- If measured by the $\mathrm{F}_{4}$ measure, trAdaBoost models slightly outperform RandomForest models trained on the combined data set without transfer learning, and these two type of models outperform other models.

\subsection{Summary of the Results and Discussion}

From the results presented above it follows that there is no "best model" that consistently outperformed the other models. Rather, different models were the best for different cases, as summarized in Table 7.4. Specifically, for within-survey predictions, when the training data were limited (i.e., $\leq 20$ pulsars), SPEGnet models outperformed RandomForest and AdaBoost models, which shows that the new representations of the data, learned by the deep neural network from the hand-crafted features, improved the separation of astrophysical SPEGs from non-astrophysical SPEGs. However, when there was an adequate amount of training data (i.e., $>20$ pulsars) this advantage diminished and the best within-survey classification models (measured by the $\mathrm{F}_{4}$ measure) were RandomForest for the PALFA-extended benchmark data set and AdaBoost for the GBTDrift benchmark data set.

For cross-survey prediction with transfer learning, when the training data were limited (i.e., $\leq 20$ pulsars) trAdaBoost performed the best when GBTDrift was used as the source data set and PALFA-extended as the target data set, whereas fine-tuned SPEGnet models worked the best when PALFA-extended was used as the source data set and GBTDrift as the target data set. When sufficient amount of training data were available ( $>20$ pulsars) there was no need of using transfer learning because the models trained only on the target data sets worked well.

\begin{tabular}{|lll|lll|}
\hline \multicolumn{2}{|c|}{ Within-survey } & \multicolumn{3}{c|}{ Cross-surveys with transfer learning } \\
\hline $\begin{array}{l}\text { Source training } \\
\text { pulsars }\end{array}$ & $\begin{array}{l}\text { PALFA- } \\
\text { extended }\end{array}$ & GBTDrift & $\begin{array}{l}\text { Target training } \\
\text { pulsars }\end{array}$ & PALFA- \\
extended & GBTDrift \\
\hline 20 & SPEGnet & SPEGnet & $\leq 20$ & trAdaBoost & ft- \\
& & & & SPEGnet \\
& & & & Transfer learning not needed \\
\hline
\end{tabular}

The fact that there is no single best model is not surprising, given the "No Free Lunch" theorem [110]. That is, a model that explains a certain situation well may fail in another situation. Therefore, it is common in machine learning to try multiple models and find one that works best for a particular situation. This is especially true in supervised learning. Nonetheless, a closer examination of the differences between the 
two benchmark data sets helped us to understand the performance differences of models on these two data sets and sheds some light on how to further improve the models in future.

Specifically, the number of non-astrophysical SPEGs identified in a beam from the PALFA survey is significantly higher than those identified in a beam from the GBTDrift survey. Specifically, as shown in Table. 5.2, although the observation length of a PALFA beam is almost twice as long as a GBTDrift beam, the total number of identified SPEGs in the PALFA-extended benchmark is 4.7 times as many as those identified in the GBTDrift benchmark. In contrast, the total number of astrophysical SPEGs detected in the PALFA-extended benchmark is only 2.5 times as many as those detected in the GBTDrift benchmark.

Furthermore, because the smearing in time due to an incorrect DM is strongly frequency dependent, DM channels are more densely spaced for data observed at lower frequencies [89]. However, the $\mathrm{S} / \mathrm{N}$ will also fall off with $\mathrm{DM}$ more rapidly so that SPEGs of similar brightness at each frequency would have roughly the same number of points. In addition, because $\mathrm{S} / \mathrm{N}$ falls off more rapidly with $\mathrm{DM}$ at low frequencies, it is much easier to discriminate astrophysical signals from RFI at $350 \mathrm{MHz}$ than at $1.4 \mathrm{GHz}$. Such a difference may have a side effect on the transfer learning. For example, by removing features that have significantly different distributions between the two benchmarks (such as DM width), the results of transfer learning may be improved. On the other hand, removing the DM width feature may result in misclassification of very wide or very narrow pulses that look different from most pulses. Note that the feature selection for transfer learning [102] is out of the scope of this paper and will be explored in our future work.

Even though the PALFA survey is more sensitive to dim and distant pulsars [99], the number of labeled low $\mathrm{S} / \mathrm{N}$ pulsars included in the GBTDrift benchmark was actually higher than those in the PALFA-extended benchmark. This is because the low $\mathrm{S} / \mathrm{N}$ detections of pulsars in the GBTDrift survey could be more easily verified and labeled (without the need of follow-up observation) due to the drift-scan nature of the GBTDrift survey. In a drift-scan survey, the telescope remains fixed and the data are split into $\sim 140$ s pseudo-pointings, each overlapping the preceding one by $\sim 70 \mathrm{~s}[61]$. This increased the chance of pulsars being detected in multiple beams with varied flux density on different days, naturally augmenting the data and increasing the diversity of pulses, which resulted in better generalization of the training of models. In some cases, we were 
able to include a few low S/N pulsars with only one astrophysical SPEG detected, but we could not do this for the PALFA-extended benchmark as the PALFA survey covered regions of the sky by observing predetermined pointings, in which most pulsars are only detected in a single beam except for very bright pulsars [99].

To show the differences between the two benchmark data sets quantitatively, we explored the distribution differences of SPEGs with two important features: number of labeled astrophysical SPEGs observed within a beam (with astrophysical pulses observed) and the peakS/N of the brightest labeled astrophysical SPEG within a beam. As can be seen in Figure 7.5, the GBTDrift benchmark data set had a much higher portion of beams that contained fewer than four labeled astrophysical SPEGs than the PALFA-extended benchmark data set (i.e., 27.5\% compared to only 9.2\%) ${ }^{2}$. Furthermore, as shown in Figure 7.6, the GBTDrift benchmark data set had significantly more beams containing only dim labeled astrophysical SPEGs (with peak $S / N$ of the brightest labeled SPEG < 10.8) than the PALFA-extended benchmark data set (i.e., $50 \%$ versus $32.5 \%$ ).

The distribution of the number of labeled astrophysical SPEGs observed in a beam (with astrophysical pulses detected) and that of the peak $S / N$ of the brightest labeled astrophysical SPEG within the beam of the benchmark data sets indicate that in the PALFA-extended benchmark data set, low S/N pulsars with only a few astrophysical SPEGs were rare cases and formed a subconcept within the pulsar class as shown in Figure 2.10. (Note that low $\mathrm{S} / \mathrm{N}$ pulses are not necessarily from dim pulsars as the $\mathrm{S} / \mathrm{N}$ of detected astrophysical pulses also depends on how far away the telescope is pointed from the source.) As a result, models learned on PALFA-extended suffered more from the within-class imbalance. Specifically, AdaBoost, which is more sensitive to rare cases than SPEGnet and RandomForest, was more likely to generate small disjuncts in the learned decision boundaries (see Figure 2.10). Meanwhile, it is observed that small disjuncts oftentimes have higher error rate than large disjuncts [107]. Therefore, AdaBoost models consistently had higher FPR values. By contrast, because RandomForest is immune to overfitting, it produced models with much lower FPR although their Recall values were also lower. As the size of training data increased, the performance of all models increased, and RandomForest eventually surpassed the other two and became the best learner for the PALFA-extended benchmark. In contrast, because the GBTDrift benchmark contained more low S/N pulsars with only one or two astrophysical SPEGs, the problem

\footnotetext{
${ }^{2}$ Note that the intervals of histogram are left-closed (right open), which means that each break point belongs to the corresponding interval on its left side.
} 

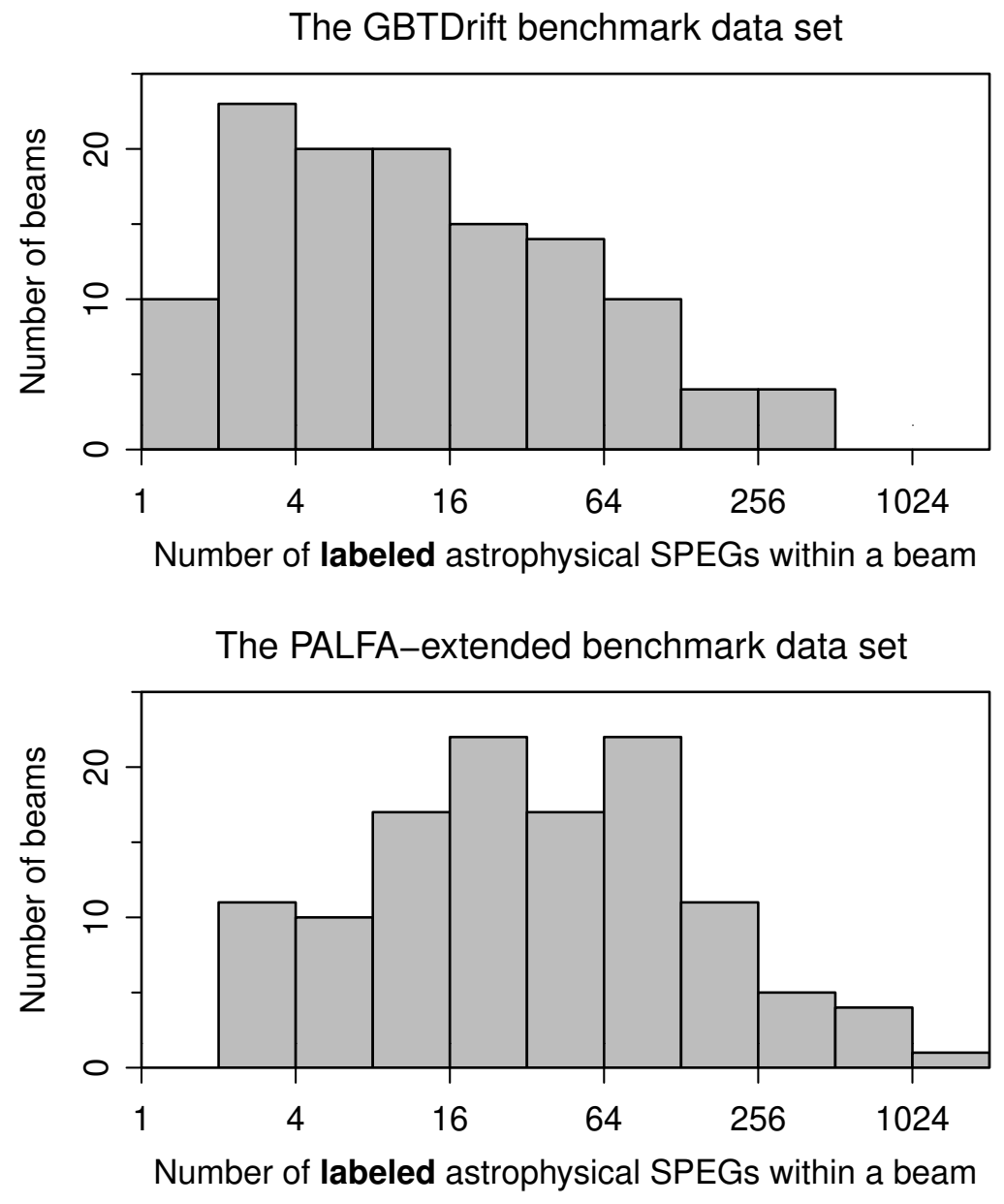

Figure 7.5: Histograms of the number of astrophysical SPEGs within in a beam from the GBTDrift and PALFA benchmark data sets. Note that the intervals of the histograms are left-closed (right open), i.e., the intervals of the histograms are: $[1,2),[2,4),[4,8)$, etc.

of overfitting was less severe, and AdaBoost was the best learner when the training data were abundant. Moreover, learners generally produced better models when they were trained on more evenly distributed GBTDrift benchmark.

These distribution differences also explain why the two transfer learning techniques performed differently when they were applied to the two benchmark data sets. When the PALFA-extended benchmark data set was considered as target, because the GBTDrift benchmark data set contained many low $\mathrm{S} / \mathrm{N}$ pulsars, it formed a good complement for PALFA-extended, and therefore the instance-based transfer learning method (trAdaBoost) outperformed the parameter-base transfer learning method (fine-tuning). In 

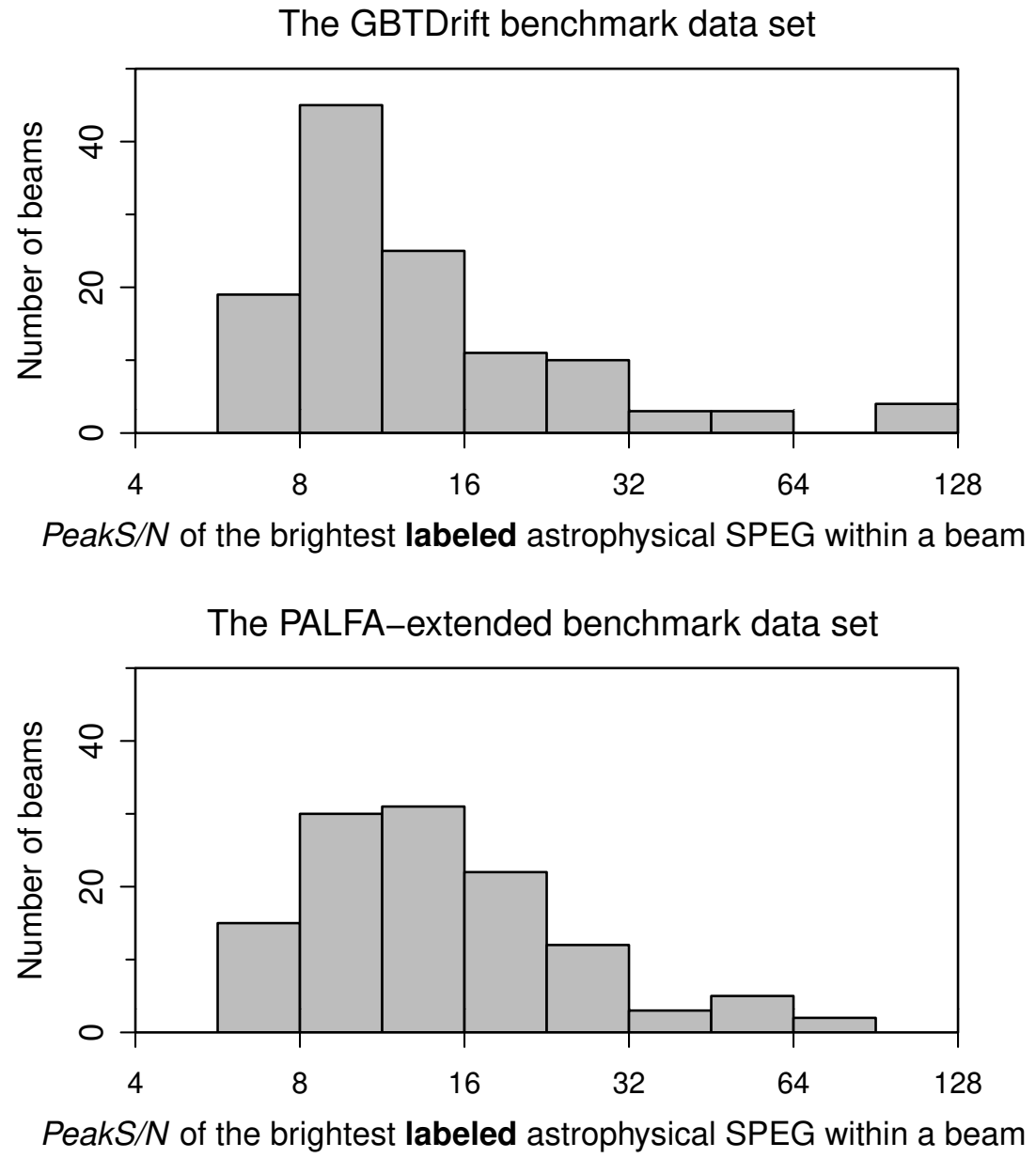

Figure 7.6: Histograms of the S/N of the brightest astrophysical SPEG within a beam from the GBTDrift and PALFA benchmark data sets. Note that the intervals of the histograms are left-closed (right open).

contrast, when the GBTDrift benchmark data set was considered as target, PALFAextended did not form a good complement. Consequently, as a parameter-based transfer learning technique, fine-tuning which extracted and retained the information from the source data set by freezing the lower layers, was less affected by the within-class imbalance problem present in the source data set compared to the instance-based method.

In summary, because in the PALFA survey only the predetermined pointings in the sky were observed, it could not be easily verified whether those detected dim signals without repetitions were of astrophysical origin or not. Therefore, when the PALFA-extended benchmark data set was created, only a few low S/N pulsars could be included. Thus, the majority of the pulsars in the benchmark data set were high $\mathrm{S} / \mathrm{N}$ pulsars, with many 
SPEGs detected. Therefore, the models trained on PALFA-extended benchmark data were not good at detecting low S/N pulsars with only a few SPEGs (i.e, rare cases). Furthermore, the lack of low S/N pulsars in the training data also caused SPEGnet and AdaBoost to produce small disjuncts, which usually have higher error rate than large disjuncts, hence causing the problem of overfitting. Consequently, RandomForest produced the best models because it is not as susceptible to overfitting. In contrast, due to the larger beam size, repeated scanning of the same sky region, and low RFI contamination of the GBTDrift survey, many low $\mathrm{S} / \mathrm{N}$ detections of known pulsars could be verified fairly easily because there were additional similar signals detected at the same position in other beams on multiple days. This resulted in the more balanced distribution of the GBTDrift benchmark and helped solve the problem of recognizing low S/N pulsars. Consequently, SPEGnet and AdaBoost performed very well on the GBTDrift benchmark and both outperformed RandomForest. In addition, our results showed that although the GBTDrift and PALFA surveys differ significantly from each other as they adopt different observing frequencies, telescopes, etc., transfer learning that combined the limited number of training instances from the target data set with the training instances from the other survey led to successful cross-survey pulsar classification.

\subsection{Conclusion}

The main implications of our finding to future studies focused on pulsar detection include: In this chapter, we investigated pulsar classification across surveys. We found when GBTDrift data were used as the target data set, the fine-tuned SPEGnet model had the highest $\mathrm{F}_{4}$ measure. On the other hand, trAdaBoost had the highest $\mathrm{F}_{4}$ measure when PALFA-extended data were used as the target data set. Our results shows the within-class imbalance had a more severe impact on instance-based transfer learning than parameter-based transfer learning. 


\section{Chapter 8}

\section{Detection of Rare Signals Using a Novel Hybrid Recommender System}

As shown in Chapter 7, pulsar classification models trained on the GBTDrift benchmark data set performed well because the benchmark data set included a variety of training pulsars with different characteristics. On the other hand, due to the lack of similar examples in the training data, pulsar classification models trained on the PALFAextended benchmark data set performed poorly on low $\mathrm{S} / \mathrm{N}$ pulsars with only several astrophysical SPEGs and pulsars with very low DM. In this chapter, we propose a hybrid recommender system that is designed to return similar rare cases in the data based on inferred user interest ${ }^{1}$.

Previous studies have found that some astrophysical signals are more difficult to find than others, in both manual and automated approaches. Examples include low $\mathrm{S} / \mathrm{N}$ pulsars [64, 79], Rotating Radio Transients (RRATs) [68], and Fast Radio Bursts (FRBs) [58]. RRATs and FRBs emit astrophysical signals that share many characteristics with pulsar signals, but their pulses are much less frequent and may have originated from other types of astrophysical objects or phenomena. Compared to bright pulsar signals, the number of these rare signals is even more limited. Therefore, machine learning approaches oftentimes rely on pulses from known pulsars as a proxy class in order to find these types of astrophysical signals [34, 80].

Ideally, a machine learning algorithm that is specifically designed for detection of

\footnotetext{
${ }^{1}$ The work presented in this chapter was published in [81].
} 
rare cases can be more efficient than standard supervised and semi-supervised learning. Furthermore, once a rare case is found, the question about whether similar candidates exist in the data set arises naturally. Furthermore, one common scenario at the stage of manual inspection is that once a rare candidate is found (whether it is pulsar, RRAT or FRB), a question about whether similar candidates exist in the database naturally arises. Therefore, we hypothesise that a recommender system that helps users find similar cases will suit this purpose well. Furthermore, the goal of pulsar searching is not only to separate pulsar signals from non-pulsar signals (i.e., classification), but also to identify credible candidates that are worthy of further investigation (e.g., re-observation by a radio telescope to collects more data for these candidates). A recommender system is well suited for addressing the latter goal. Today, recommender systems are successfully used to help users find items that may be of interest to them, such as movies, music, electronics, academic papers, and houses [2]. Yet, to the best of our knowledge recommender systems have not been applied to the pulsar candidate selection problem.

\subsection{Example of Rare Cases in Pulsar Searches}

Most pulsar signals are fairly dim when they reach Earth, which causes the detectability of pulsars to be very sensitive to the variations in telescope gain within the power pattern of a single beam or across the beams of a multibeam receiver $[23,16]$. In other words, when the pulsar is even slightly off the center of the beam, the strength of its signals will fall quickly and eventually become undetectable. Consequently, the number of detected low $\mathrm{S} / \mathrm{N}$ pulsars is extremely low because these pulsars can be easily missed in both manual and automated pulsar searches.

An example of a low $\mathrm{S} / \mathrm{N}$ pulsar is shown in Figure 8.1. As can seen, the pulsar signals are very dim and they share many characteristic of noise, which causes the problem of class overlapping. Such low S/N detection candidates have to be re-observed by the telescope for a longer period to collect more data before they are confirmed to be astrophysical sources. Additionally, the numbers of other astrophysical signals, such as pulsars with very narrow pulses, RRATs, and FRBs, are also limited [24]. Due to the small numbers of these rare cases (i.e., the within-class imbalance), it is more difficult to learn and to generalize from such signals. In this chapter, we focus on detection of two types of rare cases: low $\mathrm{S} / \mathrm{N}$ pulsars with only several pulses observed and FRBs with only one pulse observed. For this study, we use the PALFA-extend benchmark data set that was derived from the PALFA survey $[23,80]$. Because the initial benchmark data 
Chapter 8. Detection of Rare Signals Using a Novel Hybrid Recommender System 133
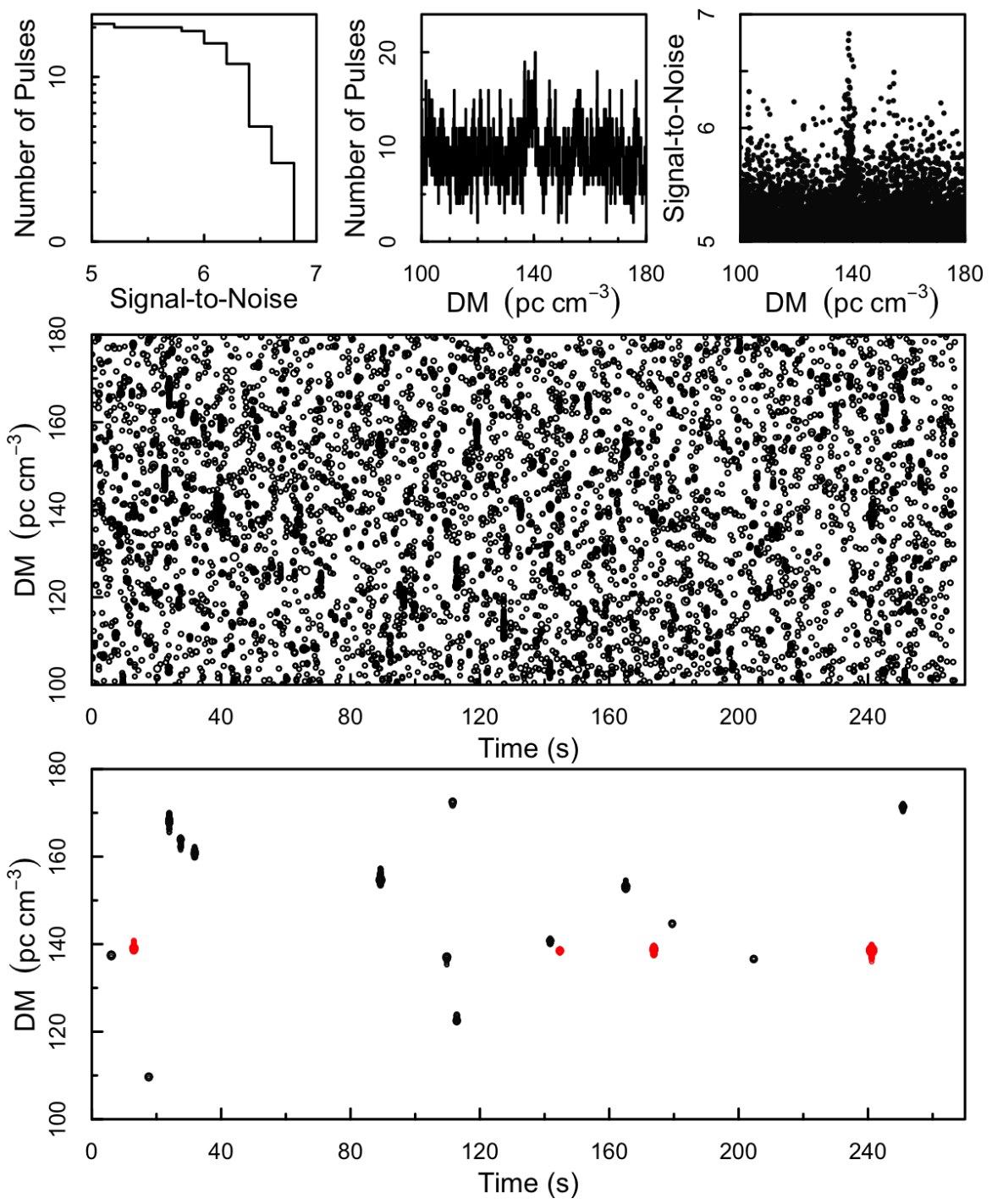

Figure 8.1: Detection of a low S/N pulsar J1957+2831 in the PALFA survey on MJD 57024. The bottom DM versus time subplot only contains pulse candidates identified by our SPEGID algorithm [79]. The pulse candidates plotted in red are confirmed as they have similar DM and underlying periodicity as pulsar J1957+2831.

set only contains several low $\mathrm{S} / \mathrm{N}$ pulsars and no FRBs, we simulated these two types of rare cases by removing some astrophysical pulses detected in the corresponding beams and created two benchmark data sets for our experiments - one with additional low $\mathrm{S} / \mathrm{N}$ pulsars and another with FRBs. (For details see section 8.3.) For each SPEG (i.e., pulse candidate) in the two benchmark data sets, we extracted the features listed in Table 8.1. 
Table 8.1: List of features extracted for each SPEG and used by the recommender system.

\begin{tabular}{|c|c|c|c|}
\hline & Feature & Description & $\begin{array}{l}\text { Data } \\
\text { Type }\end{array}$ \\
\hline 1 & ClusterDensity & Density of clusters in the DM versus time space of this particular beam. & Numerical \\
\hline 2 & PeakS/N & Maximum $\mathrm{S} / \mathrm{N}$ of the SPEG. & Numerical \\
\hline 3 & PulseWidth & $\begin{array}{l}\text { Width of the brightest single-pulse event within the SPEG, obtained from } \\
\text { matched filtering. }\end{array}$ & Numerical \\
\hline 4 & CenterDM & $\begin{array}{l}\text { For a regular SPEG, the DM of the peak } \mathrm{S} / \mathrm{N} \text {; for a clipped SPEG, the } \\
\text { determined central DM. }\end{array}$ & Numerical \\
\hline 5 & DMWidth & SPEG's width in DM (maximum DM of the SPEG minus minimum DM). & Numerical \\
\hline 6 & TimeWidth & $\begin{array}{l}\text { SPEG's width in time (maximum time of the SPEG minus minimum } \\
\text { time). }\end{array}$ & Numerical \\
\hline 7 & Clipped & Boolean value representing whether the SPEG is clipped. & Boolean \\
\hline 8 & $S I_{\mathrm{DM}}$ & $\begin{array}{l}\text { Numerical value measuring the symmetry of the SPEG by DM, SI stands } \\
\text { for Symmetry Index. }\end{array}$ & Numerical \\
\hline 9 & $S I_{\mathrm{S} / \mathrm{N}}$ & Numerical value measuring the symmetry of the SPEG by S/N. & Numerical \\
\hline 10 & PeakScore & Peak score of the SPEG. & Numerical \\
\hline 11 & SPEGRank & Rank of the SPEG by PeakS/N within the beam. & Ordinal \\
\hline 12 & WidthRatio & Ratio of TimeWidth over PulseWidth. & Numerical \\
\hline 13 & SizeRatio & $\begin{array}{l}\text { Ratio of number of single-pulse events in the SPEG over number of DM } \\
\text { channels in which they are detected. }\end{array}$ & Numerical \\
\hline 14 & GroupMaxS/N & Peak S/N of brightest SPEG in the group. & Numerical \\
\hline 15 & GroupRank & Rank of SPEG group by GroupMaxSNR within the beam. & Ordinal \\
\hline 16 & GroupMedianS/N & Median of the peak S/N of all SPEGs in the group. & Numerical \\
\hline 17 & BrRecurTimes & Number of bright SPEGs in the group. & Numerical \\
\hline 18 & RecurTimes & Total number of SPEGs in the group. & Numerical \\
\hline 19 & $S P E G s_{\mathrm{RFI}}$ & Number of SPEGs appearing at close time and lower DM. & Numerical \\
\hline 20 & $S P E G s_{\mathrm{BrRFI}}$ & Number of brighter SPEGs appearing at close time and lower DM. & Numerical \\
\hline
\end{tabular}

\subsection{Our Proposed Hybrid Recommender Systems}

In this chapter we propose a recommender system that, given any SPEG query (i.e., target), is able to retrieve a list of similar SPEGs from the data set, with the most similar SPEGs ranked at the top of the list. As each SPEG is represented as a vector of its features, in order to retrieve relevant results, it is essential to define a proper similarity measure which considers the varied importance of different features. A commonly used similarity function between the target $\bar{T}$ and a candidate $\bar{X}$ is calculated as the weighted sum of individual feature similarities in the following way [2]: 


$$
f(\bar{T}, \bar{X})=\frac{\sum_{i \in S} w_{i} \cdot \operatorname{Sim}\left(t_{i}, x_{i}\right)}{\Sigma_{i \in S} w_{i}}
$$

where $w_{i}$ is the weight of the $i$ th feature which represents its relative importance, and $\operatorname{Sim}\left(t_{i}, x_{i}\right)$ is the similarity metric between the $i$ th feature values of the specified target and candidate. Although this equation has been widely used to measure similarity between items in case-based recommender systems, when it is used for rare case detection, both the similarity metric and the weights should be modified, which is the focus of the work presented in this chapter. Inspired by [13], we propose to calculate the feature importance in our rare case detection approach as a product of two factors: the dependency measure between the feature and the class label, and the outlyingness of the feature. Furthermore, we experiment with two different dependency coefficients (DC) and explore if one works better than the other for our data sets.

As shown in Figure 8.2, our proposed feature-weighting method consists of the following steps:

- Compute the similarity metric between feature values of the target and each candidate.

- Compute the dependence coefficient (DC) for each feature in two different ways:

- Option 1: Mutual Information (MI) [94];

- Option 2: Correlation coefficient $\phi_{k}[4]$.

- Compute the outlier score of the target values.

- Compute each feature weight as a product of dependence coefficient and the outlier score.

- Compute the weighted similarity between the target and candidates and sort the candidates based on their similarity with the target.

Because we use target SPEG as an example to state our requirements (i.e., casebased method) and use labelled benchmark to learn the dependency between features and classes (i.e., content-based method), our system constitutes a hybrid recommender system. In the following subsections, we present the proposed calculation for each of the steps of our approach. 
Chapter 8. Detection of Rare Signals Using a Novel Hybrid Recommender System 136

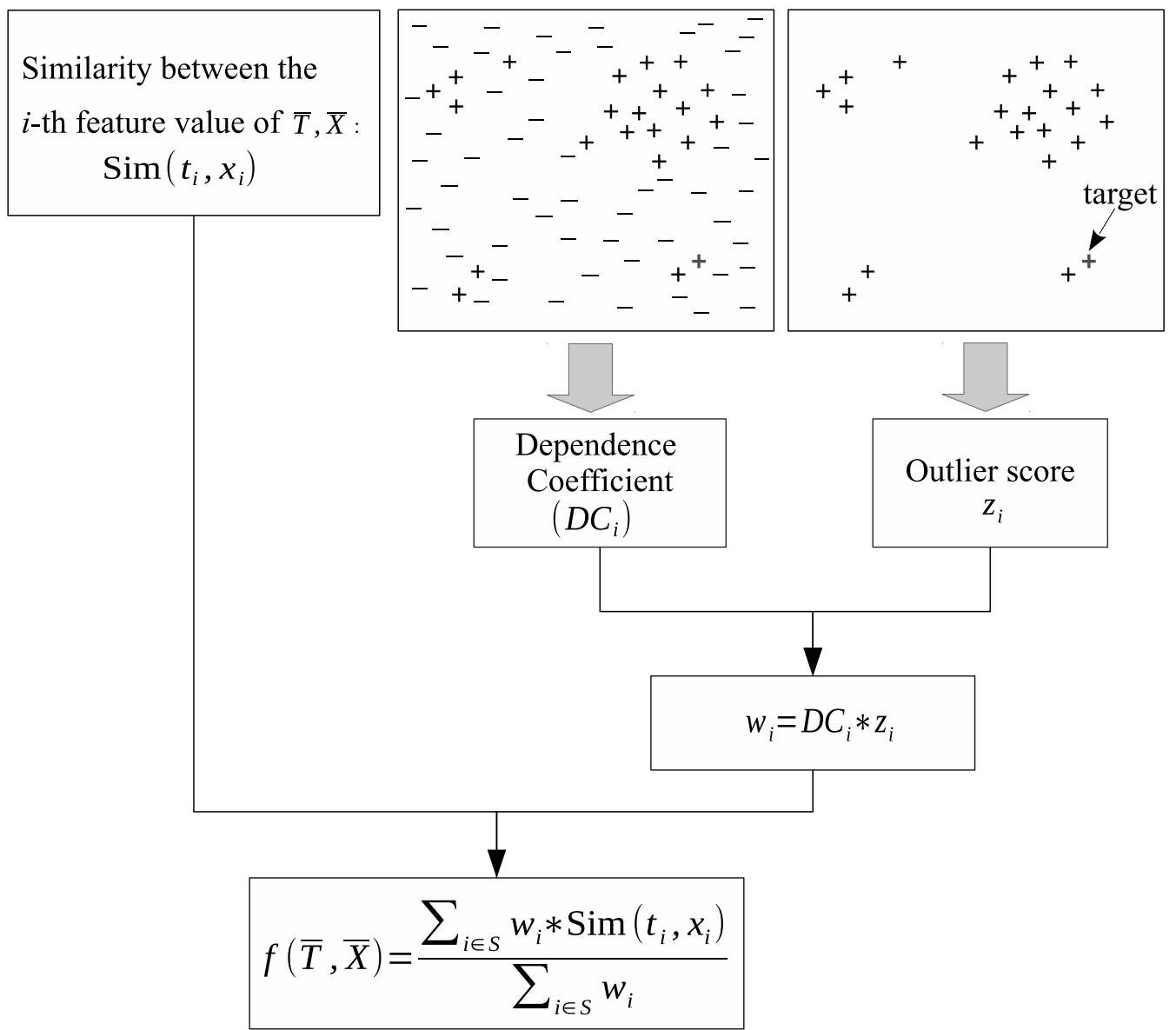

Figure 8.2: Proposed approach that calculates the weighted similarity between the target $\bar{T}$ and the candidate $\bar{X}$. Note that when we calculate the outlier score of a feature, only positively labeled SPEGs are considered.

\subsubsection{Similarity Metric Between Feature Values}

In the pulsar candidate selection problem, every candidate is represented as a vector of features, which can be Boolean, ordinal, or numerical (see Table 8.1). As a result, we define similarity metrics that work for features of different data types.

For Boolean or categorical features, we use the overlap measure [88] to calculate the similarity metric between two values:

$$
\operatorname{Sim}\left(t_{i}, x_{i}\right)= \begin{cases}1 & \text { if } t_{i}=x_{i} \\ 0 & \text { otherwise }\end{cases}
$$

where $t_{i}$ and $x_{i}$ represent the values of feature $i$ for the target and the compared candidate, respectively. 
For numerical features, we calculate the similarity between the two values as follows [2]:

$$
\operatorname{Sim}\left(t_{i}, x_{i}\right)=1-\frac{\left|t_{i}-x_{i}\right|}{\max _{i}-\min _{i}}
$$

where $\max _{i}$ and $\min _{i}$ represent the maximum and minimum values of the feature $i$ respectively. Specifically, we use Equation 8.3 to compute the similarity metric for features with uniformly distributed values. For features with heavily skewed right tail (i.e., features with extremely large values, such as features 19 and 20), we replace the maximum value $\max _{i}$ in Equation 8.3 with the $95 \%$ quantile.

For features that have symmetric distribution with a central peak, the similarity metric could be computed as:

$$
\operatorname{Sim}\left(t_{i}, x_{i}\right)=\max \left\{0,1-\frac{\left|t_{i}-x_{i}\right|}{3 \cdot \sigma_{i}}\right\}
$$

where $\sigma_{i}$ represents the standard deviation. However, since both the mean and the standard deviation are very sensitive to even one aberrant value (i.e., their breakdown value is both 1 [95]), we replace $\sigma$ in Equation 8.4 with MAD given by

$$
\mathrm{MAD}=1.483 \underset{i=1, \ldots, n}{\operatorname{median}}\left|x_{i}-\underset{j=1, \ldots, n}{\operatorname{median}}\left(x_{j}\right)\right|
$$

and instead of Equation 8.4 use the following similarity metric:

$$
\operatorname{Sim}\left(t_{i}, x_{i}\right)=\max \left\{0,1-\frac{\left|t_{i}-x_{i}\right|}{3 \cdot \mathrm{MAD}}\right\}
$$

In cases when the distribution of feature values is asymmetrical, an asymmetric reward may be appropriate. Because more SPEGs detected at similar DM often indicates larger probability of these SPEGs originating from astrophysical sources, for features 17 and 18 we propose to use an asymmetric function to reflect our preference for larger values:

$$
\operatorname{Sim}\left(t_{i}, x_{i}\right)=1-\frac{\left|t_{i}-x_{i}\right|}{\max _{i}-\min _{i}}+\alpha_{i} \cdot I\left(x_{i}>t_{i}\right) \cdot \frac{\left|t_{i}-x_{i}\right|}{\max _{i}-\min _{i}}
$$

where $\alpha_{i}=0.5$ to slightly lessen the penalty on larger values.

Note that before computing the similarity metric we apply log transformation to all features (except features 7, 10, 19 and 20) due to the following reasons: (i) to reduce the skewness of the distribution; (ii) for pulse candidates, if the feature value is small, even a moderate deviation should be considered significant; but for a large feature value, only a large deviation would be considered significant. Then, we calculate the similarity metric between feature values using Equation 8.2 for feature 7; Equation 8.3 for features 10, 13, 15, 19, and 20; Equation 8.6 for features 1-6, 8, 9, 11, 12, 14, and 16; and Equation 8.7 for features 17 and 18 . 


\subsubsection{Weighting of Features in our Hybrid Recommender System}

As shown in Figure 8.2, weighting of features in our hybrid recommender system is computed as a product of the dependence coefficient and the outlier score. Because SPEG features are of various data types, a proper dependence coefficient must be measured between different types of variables (numerical, ordinal and categorical). We propose to use two different dependence coefficients, namely Mutual Information (MI) and Correlation Coefficient $\phi_{k}$ to calculate the dependence between each feature and the class label. Using an outlier score $z$ for each feature has potential to improve user's interest inference because when the goal is to find similar rare cases among large number of candidates, the rarity of target feature values may contain information related to user's interest. Next, we describe the proposed ways to calculate the two different dependence coefficients in Sections 8.2.3 and 8.2.3, and the outlier score in Section 8.2.4.

\subsubsection{Calculation of Dependence Coefficient}

\section{Mutual Information (MI)}

For a random variable $X$, the entropy (denoted as $H(X)$ ), which is used to quantify the uncertainty in its distribution, is defined as:

$$
H(X)=-\sum_{x} p(x) \log p(x)
$$

where $0 \leq p(x) \leq 1$ for any event $x$. If $x$ is an impossible event, $p(x)=0$.

MI is used to measures the degree of relatedness between two variables $X$ and $Y$ and can be computed from the statistics of the $(x, y)$ pairs. MI between two discrete variables can be calculated using the following equation:

$$
\begin{aligned}
I(X, Y) & =H(Y)-H(Y \mid X) \\
& =\sum_{x} \sum_{y} p(x, y) \log \frac{p(x, y)}{p(x) p(y)} .
\end{aligned}
$$

For two continuous variables or variables of mixed data types, we propose to compute MI using the nearest-neighbors non-binning estimator [94], which is more robust than grouping the continuous variable into discrete "bins" and using Equation 8.9. Specifically, we compute the MI between discrete and continuous variables as follows. First, for each data point $i$, a number $I_{i}$ is calculated based on its nearest neighbor in the continuous variable $Y$ :

$$
I_{i}=\Psi(N)-\Psi\left(N_{x_{i}}\right)+\Psi(k)-\Psi\left(m_{i}\right)
$$


where and $\Psi(\cdot)$ is the digamma function, $N$ is the total number of data points, $x_{i}$ is the value of the discrete variable $X$, hence $N_{x_{i}}$ is the number of data points having the value of $x_{i}, \mathrm{k}$ is the parameter that represents the number of nearest neighbors, and $m_{i}$ is the number of data points in the full data set that fall within the neighborhood. MI is then estimated by averaging $I_{i}$ over all data points:

$$
I(X, Y)=\left\langle I_{i}\right\rangle=\Psi(N)-\left\langle\Psi\left(N_{x}\right)\right\rangle+\Psi(k)-\langle\Psi(m)\rangle
$$

\section{Correlation Coefficient $\phi_{k}$}

Although Pearson's correlation coefficient is commonly used to measure the correlation between paired variables, it only works for numerical variables. To calculate correlations between variables of mixed types (e.g., categorical, ordinal and numerical), recently a new correlation coefficient $\phi_{k}$ was proposed [4]. To calculate $\phi_{k}$, a contingency table of two variables is first created, and the Pearson's $\chi^{2}$ value is interpreted as coming from a bivariate normal distribution $\left(f_{\text {b.n. }}\right)$ with correlation parameter $\phi_{k}$. Consequently, $\phi_{k}$ is calculated by inverting the $\chi^{2}$ contingency test statistic. Note that binning is needed when the variable is continuous. First, the $\chi^{2}$ contingency test statistic is calculated as follows:

$$
\chi_{\text {b.n. }}(\rho, N, r, k)=N \sum_{i, j}^{k, r} \frac{\left(F_{i j}(\rho=\rho)-F_{i j}(\rho=0)\right)^{2}}{F_{i j}(\rho=0)}
$$

where $\rho$ is the correlation coefficient of the bivariate normal distribution, $N$ is the total number of data points, $r$ and $k$ are the number of rows and columns of the contingency table, respectively, and $F_{i j}(\rho)$ is the integral of the bivariate normal distribution over the area of bin $i j$ :

$$
F_{i j}(\rho)=\int_{\text {area }_{i j}} f_{\text {b.n. }}(x, y \mid \rho) \mathrm{d} x \mathrm{~d} y .
$$

To account for statistical noise, a sample-specific pedestal is defined as

$$
\chi_{\text {ped }}^{2}=n_{\text {sdof }}+c \cdot \sqrt{2 n_{\text {sdof }}}
$$

where $n_{\text {sdof }}$ is the the effective number of degrees of freedom of the bivariate sample:

$$
n_{\text {sdof }}=(r-1)(k-1)-n_{\text {empty }}(\text { expected }) .
$$

$\chi_{\max }^{2}$ is the maximum possible $\chi^{2}$ value of the contingency test:

$$
\chi_{\max }^{2}=N \min (r-1, k-1)
$$


Equation 8.12 is then scaled to ensure it equals $\chi_{\text {ped }}^{2}$ for $\rho=0$ and $\chi_{\max }^{2}$ for $\rho=1$ :

$$
\chi_{\text {b.n. }}(\rho, N, r, k)=\chi_{\text {ped }}^{2}+\left\{\frac{\chi_{\text {max }}^{2}(N, r, k)-\chi_{\text {ped }}^{2}}{\chi_{\text {b.n. }}(1, N, r, k)}\right\} \cdot \chi_{\text {b.n. }}^{2}(\rho, N, r, k)
$$

The correlation coefficient $\phi_{k}$ is defined as the solution for $\rho$. $\phi_{k}$ differs from the Pearson correlation coefficient in the following ways: (i) $\phi_{k}$ has a range of $[0,1]$ and hence it cannot distinguish positive and negative association values; (ii) $\phi_{k}$ is capable recognizing nonlinear dependency. When the input data are from a bivariate normal distribution and the variables are uniformly binned, $\phi_{k}$ reverts to the Pearson correlation coefficient.

\subsubsection{Improving User's Interest Inference Using an Outlier Score}

When a specific target is selected, its characteristics captured by its feature vector to a large extent reflect user's interest. This combined with dependence coefficients that assign larger weights to more important features can theoretically help us retrieve similar items. Nevertheless, when the target is a rare case and the number of candidates is large, the rarity of the feature values may be an indicator of user interest. In other words, assigning larger weights to rare feature values can be beneficial for finding similar rare cases. Therefore, we propose to treat rare feature values of the target as outliers among the positive class and use the outlier score of the features to improve user's interest inference. Note that only positive examples are used to calculate the outlier score, which is different from the calculation of similarity metric in Section 8.2.1 where both positive and negative training examples are used.

For numerical variables, we use $z$-score to represent the outlyingness of their values. Specifically, we use the median and the median of all absolute deviations from the median (MAD) as robust measures of location and scale, respectively [95]:

$$
z_{i}=\left(x_{i}-\underset{j=1, \ldots, n}{\operatorname{median}}\left(x_{j}\right)\right) / \mathrm{MAD}
$$

For a numerical variable that is normally distributed, its outlyingness can be measured by first grouping the values into intervals and then treating the variable as a categorical variable. For categorical variables, the notion of density is defined as the fraction of data points with the same values out of all data points [88]:

$$
\operatorname{density}\left(X_{i}\right)=\operatorname{freq}\left(x_{i}\right) / n
$$


where $\operatorname{freq}\left(x_{i}\right)$ is the number of data points with value $x_{i}$ for the particular attribute $i$. In our study, we use equation (8.20) to represent the outlyingness of a categorical variable:

$$
z\left(X_{i}\right)=\log \left(\frac{\operatorname{density}\left(\operatorname{mode}\left(X_{i}\right)\right)}{\operatorname{density}\left(X_{i}\right)}\right)
$$

In summary, for features $2-6,8,9,12,14$, and 16-18 we first apply the log transformation and then calculate the outlier score using Equation 8.18; for the Boolean feature 7 and ordinal features 11 and 15, as well as the numerical features 1, 10, 13, and 19, we first bin the values and then use the Equation 8.20 to calculate their outlier score. Feature 20 was not considered because most of its values were 0 . We also set the minimum value of the outlier score to 1, as shown in Equation 8.21:

$$
z_{j}=\max \left(\left|z_{j}\right|, 1\right)
$$

\subsection{Experiments}

In this study, we use low $\mathrm{S} / \mathrm{N}$ pulsars and FRBs as target queries to test our hybrid recommender system's ability to detect rare cases. Due to their limited number, such pulsars are hard to find using supervised machine learning [80]. We conduct our experiments on two benchmarks (one for low $\mathrm{S} / \mathrm{N}$ pulsars and another for FRBs), in each case using three-fold cross validation, with two folds used as training data and the third fold as test data. Each fold consists of 40 beams with pulsars and 400 beams without pulsars (see Table 5.2). As each beam contains tens or even hundreds of SPEGs, in each fold there are about 80,000 SPEGs.

Due to the lack of low $\mathrm{S} / \mathrm{N}$ pulsars with only a few pulses detected and the lack of FRBs in the initial benchmark data, we simulated them by manipulating the real astrophysical pulses in the beams. Specifically, each simulated low S/N pulsar was limited to have three to six astrophysical pulses with $p e a k S / N$ between $6 \sigma$ and $7 \sigma$, which was archived by removing the brighter pulses. On the other side, each simulated FRB only had one astrophysical pulse with peak $S / N$ no less that $8 \sigma$, which was achieved by removing the remaining pulses. We simulated three low $\mathrm{S} / \mathrm{N}$ pulsars (or FRBs) within three beams in the training data (two folds) as target examples. We also simulated three low $\mathrm{S} / \mathrm{N}$ pulsars (or FRBs) in the test fold as rare cases to be found. In the test fold, we removed all beams containing pulsar signals except for the three beams containing simulated rare signals (because other pulsars can be easily found using supervised learning), which reduced the number of SPEGs to about 75,000. Our recommender system sorts 
the SPEGs in the test data in descending order, based on their similarity with the target SPEG. We implemented our hybrid recommender system in Python and have made both the data and the code available online ${ }^{2}$.

Because we use three-fold cross validation, the total number of rare cases (i.e., low $\mathrm{S} / \mathrm{N}$ pulsars or FRBs) in the training data and test data is nine. To avoid bias due to the selection of simulated pulsars, we randomly selected nine beams with pulsars to be used for simulation of low $\mathrm{S} / \mathrm{N}$ pulsars (or FRBs) and repeated the experiments thirty times. The results reported in Section 8.4 are averages over the thirty runs.

We experimented with five different feature-weighting schemes for detection of either low S/N pulsars or FRBs: (i) uniform weighting of features which serves the baseline; (ii) weighting of features using MI only; (iii) weighting of features using the product of MI and the outlier score $(\mathrm{MI} \cdot z)$; (iv) weighting of features using the correlation coefficient $\phi_{k}$ only; (v) weighting of features using the product of the correlation coefficient $\phi_{k}$ and the outlier score $\left(\phi_{k} \cdot z\right)$.

\subsection{Results}

\subsubsection{Detection of Low S/N Pulsars}

The results of using different feature-weighting schemes for detection of low $\mathrm{S} / \mathrm{N}$ pulsars are shown in Figure 8.3. As can be seen, weighting schemes were able to rank pulsar signals in the test data at the top of the list containing 75,000 SPEGs. Overall, weighting of features using MI, MI $\cdot z$ and $\phi_{k}$ all consistently outperformed uniform weighting at different levels of recall, hence required fewer SPEGs to be inspected. This shows assigning larger weights to more important features increased the similarity between pulsar signals. Furthermore, MI $\cdot z$ outperformed the other feature-weighting methods at most levels of recall, which shows that using rarity of feature values generally improved user's interest inference. Specifically, when combining MI and outlier score $z$, on average our system was able to retrieve 5 out of 9 low $\mathrm{S} / \mathrm{N}$ pulsars within the top 12 (out of 75,000 ) most similar candidates to each target example, and to retrieve 8 out of 9 low S/N pulsars within the top 144 (out of 75,000 ) most similar candidates of each target example. Even with uniform weighting, the recommender system was able to find the pulsars when the target pulsars and the test pulsars were very similar (e.g., to retrieve 5 out of 9 low $\mathrm{S} / \mathrm{N}$ pulsars within the top 24 most similar candidates), which proved the effectiveness

\footnotetext{
${ }^{2}$ https://github.com/dipangwvu/rare_case_detection
} 


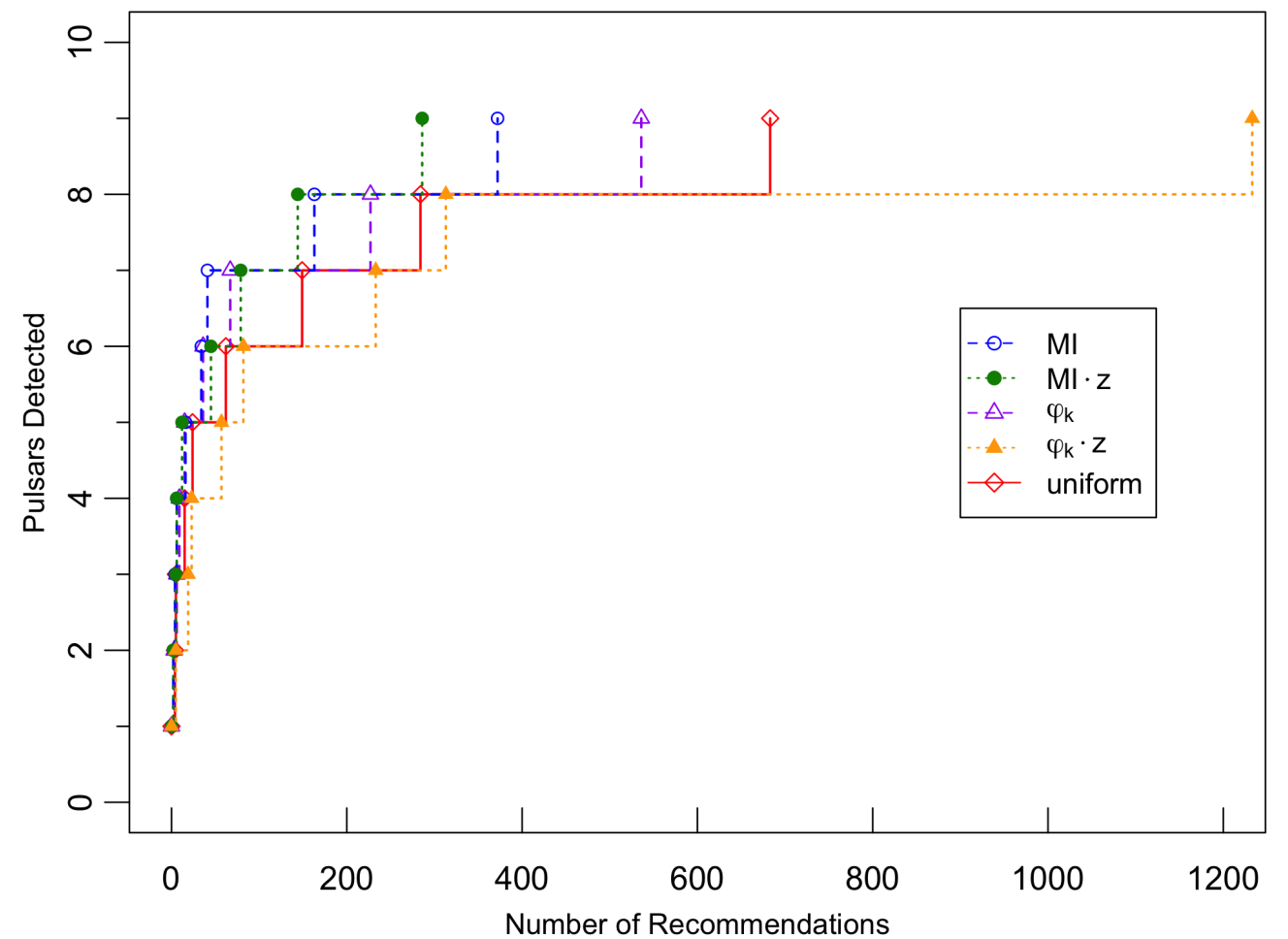

Figure 8.3: Number of low S/N pulsars detected versus number of recommendations.

of the proposed similarity metric (see Section 8.2.1). This agrees with a previous study that showed that learning in the dissimilarity space could result in better performance than learning in the feature space [38]. We also calculated the normalized discounted cumulative gain (NDCG), which is used as a measure of ranking quality. We assigned a relevance of 0 or 1 to each item and used a recommendation list size of 30 items, which is ten times the number of simulated pulsars in the test data. Consistent with the observations made using Figure 8.3, the weighting scheme (MI $z$ ) had the highest NDCG score; its low value of 0.22 illustrates the difficulties of rare cases detection when class overlapping is present.

Table 8.2 shows the most important features identified by different featureweighting schemes. The MI-based weighting schemes identified the following features as the most important: GroupMaxS/N, GroupMedian, RecurTimes, BrRecurTimes, ClusterDensity and CenteredDM. The combination of the first four features correctly captured the characteristics of our target - pulsars that consisted of several low S/N pulses. Furthermore, such candidates are more likely to be astrophysical in beams that contain less noise and RFI signals, therefore ClusterDensity is another highly ranked feature. This is likely why MI-based schemes outperform the other weighting schemes. 
Table 8.2: Ranking of features for detection of low $\mathrm{S} / \mathrm{N}$ pulsars. This rank is based on feature weights and is given in parentheses if that feature weight accounts for $<5 \%$ of the total weight.

\begin{tabular}{lcccc}
\hline & \multicolumn{4}{c}{ Method } \\
Feature & MI & MI $\cdot \mathrm{z}$ & $\phi_{k}$ & $\phi_{k} \cdot \mathrm{z}$ \\
\hline ClusterDensity & 5 & 5 & 7 & $(9)$ \\
GroupMaxS/N & 1 & 1 & 1 & 2 \\
GroupRank & $(13)$ & $(8)$ & 5 & 1 \\
GroupMedianS/N & 2 & 3 & 10 & $(11)$ \\
BrRecurTimes & 4 & 4 & 2 & 5 \\
RecurTimes & 3 & 2 & 3 & 6 \\
PeakS/N & $(14)$ & $(15)$ & 6 & $(8)$ \\
CenteredDM & 6 & 6 & 4 & $(7)$ \\
PeakScore & $(10)$ & $(7)$ & 9 & 4 \\
SPEGRank & $(18)$ & $(17)$ & $(12)$ & 3 \\
\hline
\end{tabular}

Compared with MI, MI $\cdot z$ only slightly altered the order of the top ranked features. At moderate level of recall, using MI only turned out to be the best strategy. This shows that using outlier score of feature values to infer user interest may not always be desired. For example, when the target pulsar had a very high DM, weighting of features using MI $\cdot z$ shifted many RFI SPEGs with high peak DM to the top of the recommendation list, which resulted in lower ranking for real test pulsars with low DM. Since knowledgebased recommender systems are highly customizable, this can be avoided by asking the user to manually select which features to be considered as outerliers when calculating the $z$ score. The recommendations can be further improved by applying critiques to filter SPEGs using specific constraints. For example, because we know bright pulsars with multiple pulses can be easily detected with supervised learning, when the target SPEG has low peak $S / N$, we can simply filter all SPEGs with GroupMax $S / N$ greater than $7 \sigma$ and/or RecurTimes greater than 6. This would filter bright SPEGs originating from RFI and therefore significantly reduce the number of false positives.

\subsubsection{Detection of FRBs}

In this subsection, we present the results of FRB detection. As can be seen in Figure 8.4, all feature-weighting schemes were able to rank the FRB signals at the top 


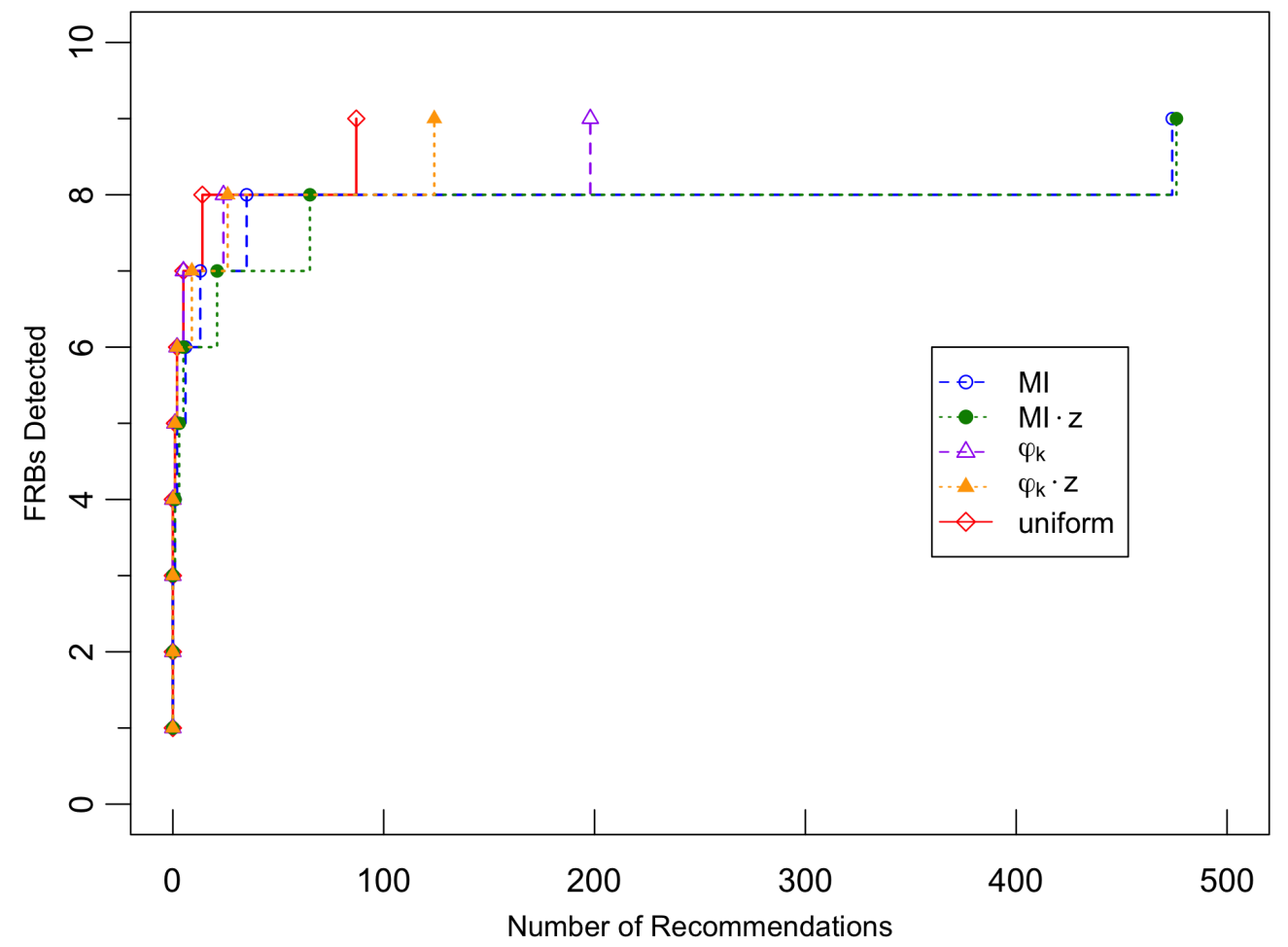

Figure 8.4: Number of FRBs detected versus number of recommendations.

of the list containing 75,000 SPEGs. Compared with detection of low S/N pulsars, there are two major differences: (i) The uniform weighting was the best strategy, and $\phi_{k}$-based methods outperformed MI-based methods, especially at high level of recall when there was obvious difference between the target and the candidate FRBs. (ii) FRBs were ranked much higher among all the candidates, thus much less recommendations needed to be inspected in order to find all the FRBs in the test data than to find all the low $\mathrm{S} / \mathrm{N}$ pulsars. Specifically, the best weighting scheme was able to retrieve 8 out of 9 FRBs on average within the top 14 (out of 75,000) most similar candidates to the target example, compared to inspecting the top 144 most similar candidates for detecting 8 out of 9 low $\mathrm{S} / \mathrm{N}$ pulsars.

Furthermore, using the same recommendation list size (i.e., 30 items), the best weighting scheme had an NDCG score of 0.61 , which is much higher than the NDCG score for low $\mathrm{S} / \mathrm{N}$ pulsars. These results indicate that the difference between FRBs and non-astrophysical signals is more distinguishable than the difference between low $\mathrm{S} / \mathrm{N}$ pulsar signals and non-astrophysical signals, which was expected as FRBs have higher $\mathrm{S} / \mathrm{N}$ and are more noticeable even in manual inspection.

Table 8.3 shows the most important features for detection of FRBs, identified by dif- 
Table 8.3: Ranking of features for detection of FRBs. This rank is based on feature weights and is given in parentheses if the feature weight accounts for $<5 \%$ of the total weight.

\begin{tabular}{lcccc}
\hline & \multicolumn{4}{c}{ Method } \\
Feature & MI & $\mathrm{MI} \cdot \mathrm{z}$ & $\phi_{k}$ & $\phi_{k} \cdot \mathrm{z}$ \\
\hline ClusterDensity & 5 & 5 & 7 & $(8)$ \\
GroupMaxS/N & 1 & 3 & 1 & 3 \\
GroupRank & $(13)$ & $(12)$ & 4 & $(4)$ \\
GroupMedianS/N & 2 & 4 & 10 & $(10)$ \\
BrRecurTimes & 4 & 2 & 2 & 1 \\
RecurTimes & 3 & 1 & 3 & 2 \\
PeakS/N & $(14)$ & $(15)$ & 6 & $(7)$ \\
CenteredDM & 6 & 6 & 5 & $(5)$ \\
PeakScore & $(10)$ & $(9)$ & 9 & $(6)$ \\
\hline
\end{tabular}

ferent feature-weighting schemes. As can be seen, the best weighting scheme among the four, $\phi_{k} \cdot \mathrm{z}$ assigns highest weights to BrRecurTimes, RecurTimes, and GroupMaxS/N and all other features account for less than 5 percent of the total weight. This finding agrees with the fact that FRBs are non-repetitive, bright pulses and likely explains why $\phi_{k} \cdot \mathrm{z}$ turned out to be the best among the four weighting schemes. The fact that the uniform weighting outperformed the all four weighting schemes indicates that FRBs have distinct features that make them easily distinguishable from noise and RFI signals. This finding supports using pulsar as proxy class for training supervised learning models to detect FRB signals in the DM versus time domain.

\subsection{Conclusion}

We proposed a novel hybrid recommender system that was designed to find rare cares in the data. Note that nearest neighborhood-based methods had been used for classification of small clusters in computational linguistics [103] and in case-based reasoning systems [49]; however, in order to apply it to detect extremely rare cases that are represented by features of different data types in the presence of class overlapping, the calculation of similarity between items had to be modified accordingly.

We proposed a similarity metric that not only accommodates features of different 
data types but also accounted for different distributions of features' values. We computed the weight of each feature as a product of its association with the class label and the outlyingness of its value. Specifically, we considered two different dependence coefficients (i.e., Mutual Information and Correlation Coefficient $\phi_{k}$ ) applicable to different data types and explore which suited our data better. Furthermore, we used the outlyingness of feature values of the target to improve the user's interest inference. We applied the proposed hybrid recommender system on detection of two types of rare cases that existed in the pulsar candidate selection: pulsars with low $\mathrm{S} / \mathrm{N}$ and FRBs.

Based on the results presented in this chapter, the main findings can be summarized as follows: (1) The proposed hybrid recommender system successfully detected pulsar signals and FRBs that were similar to the target rare cases. (2) In the case of low $\mathrm{S} / \mathrm{N}$ pulsars, as there was class overlapping in the data, treating rare feature values as outliers and increasing their weights in the similarity function helped to improve user's interest inference, hence reduced the number of pulsar candidates that required manual inspection. (3) Detection of FRBs is much easier than detection of low S/N pulsars as FRBs are less affected by the class overlapping problem. As a result, uniform weighting of features performed the best for detection of FRBs, followed by using the product of $\phi_{k}$ and outlier score. 


\title{
Chapter 9
}

\section{Conclusion and Future Work}

\author{
"Acquire new knowledge whilst thinking over the old, and you may become \\ a teacher of others."
}

- Confucius, Analects

\subsection{Look-back}

Detection of radio pulsar signals using machine learning is an interesting yet difficult problem. On one hand, it shares many properties with other real-world problems including class imbalance, large volumes of data, etc. On the other hand, it has some distinctive characteristics which separate it from other problems. The term pulsar candidate selection is used because in most cases a discovery can only be claimed after similar signals are detected in follow-up observations, or the same signal can be identified in different domains (e.g., both the time domain and the frequency domain). Moreover, because data collection is expensive, high recall is strongly preferred over high precision. Furthermore, pulsar searching using machine learning is an imbalance learning problem, yet to a certain extent, rare pulsar signals are often of greater interest than "common" pulsar signals as the former could further improve our understanding of pulsar astronomy.

In a little over a decade, pulsar searching using machine learning was consistently improved and achieved many successes. For a long time astronomers were more interested in periodicity searches, therefore machine learning was also firstly applied to periodicity searching data. Selection of credible candidates in the periodicity searches (or singlepulse searches in the frequency domain) is essentially an image classification problem as the domain expert has to decide whether each diagnostic plot represents a pulsar candidate or not. As a result, many general image classification algorithms (from feature 
engineering to deep learning) can be used for candidate selection (with necessary modifications). In contrast, single-pulse searches in the time domain are more difficult because a pulse candidate must first be separated from RFI and other pulse candidates before a decision can be made. Additionally, the high sampling rate in the time domain becomes the biggest obstacle that prevents the application of many computer vision techniques to single-pulse search candidate selection. (Another lesser obstacle is different DM spacing in different DM ranges.) In the most extreme case, a single pulse candidate can be viewed as an object made up of five pixels in an image with a resolution of 10,000 $\times$ $1,000,000$. Although downsampling in the time domain seems plausible, it will result in the loss of time resolution that can be important for finding dim periodic signals, whereas downsampling in the DM domain may result in the loss of narrow pulses completely. As a result, in this dissertation, we developed techniques that were different from computer vision methods to automatically identify and classify single-pulse candidates.

\subsection{Main Contributions}

We developed a two stage approach, namely SPEGID, that can identify and classify pulse candidates in the time domain. Specifically, we used DBSCAN clustering to group single-pulse events in the DM versus time domain and then merged the clusters based on the expected DM and time span of astrophysical pulses. This way our approach could identify pulse candidates consisting of as little as five single-pulse events, pulse candidates consisting of hundreds of single-pulse events, or pulse candidates that were clipped during the data processing step. Furthermore, we developed a peak-scoring algorithm that could identify the expected peak in the $\mathrm{S} / \mathrm{N}$ versus $\mathrm{DM}$ curve and this algorithm proved to work for narrow pulses and wide pulses, pulses with single or multiple components, and was able to filter RFI with flat $\mathrm{S} / \mathrm{N}$ versus DM curve at the same time. We then grouped the pulse candidates that were detected at close DM and extracted features from each pulse candidate and the candidate groups.

For the classification stage, we tested a wide range machine learning algorithms including decision tree learner, rule learner, SVM, ensemble learners and deep neural networks. Our models achieved high recall and low false positive rate. We further proposed a new algorithm to identify possible periodicity among single-pulse search candidates detected at close DM, and dim pulse candidates without underlying periodicity were then eliminated from further investigation. By re-analyzing the GBTDrift survey and AO327 survey data, we discovered eight pulsars that were missed in previous analysis. 
We investigated pulsar candidate classification across surveys for the first time and showed that the performance of cross-survey classification could be improved using existing transfer learning techniques. We also found that without transfer learning, a benchmark with 20 representative pulsars could achieve good results and adding more labeled pulsars into the benchmark improved the model slightly. Finally, we developed a hybrid recommender system to detect rare pulsar signals. Using simulated low $\mathrm{S} / \mathrm{N}$ pulsars and FRBs as examples, we showed that our recommender system was able to identify the most important features and detect similar rare signals in the data. In addition, using NDCG score as the performance metric, the results showed FRBs were easier to detect than low $\mathrm{S} / \mathrm{N}$ pulsars, most likely because the number of candidates increases significantly at low $\mathrm{S} / \mathrm{N}$ level.

\subsection{Future Work}

One direction of future research is to improve the computing efficiency of the algorithms. Normally single-pulse search algorithms presented in this dissertation were designed to work offline. When the number of observed beams are large (e.g., tens of thousands) it can take days for SPEGID to identify all the candidates. Big data technologies such as Spark have been successfully used for classification of pulsar candidates [27]. These technologies allow us to link a set of computers over a network into a cluster that is not limited by the number of processor cores, memory or other hardware. With efficient resource management and job scheduling/monitoring, cluster computing has many advantages such as high availability, load balancing and scaling capabilities, and performance improvements. The SPEGID algorithms can be similarly modified to run on a computing cluster and make real-time single-pulse searching possible. Moreover, because pairwise comparison is needed, it takes time for the hybrid recommender system to return the candidates that are most similar to the target signal among millions of candidates. Therefore, improving the computing speed using big data technology would benefit the hybrid recommender system as well.

Secondly, even with machine learning, the number of predicted pulse candidates is fairly large, especially at low $\mathrm{S} / \mathrm{N}$ level. Therefore, as part of future work we plan to develop techniques that can further reduce the number of false positives efficiently. For example, we will use astrophysical information about a source's sky position or other attributes to form a prior probability distribution for single-pulse properties such as DM and/or pulse width. 


\section{List of Publications}

1. Di Pang, Katerina Goseva-Popstojanova, Maura McLaughlin. "Hybrid Recommender System for Detection of Rare Cases Applied to Pulsar Candidate Selection”, 2021 IEEE 8th International Conference on Data Science and Advanced Analytics (DSAA), 1-10, 2021.

2. Di Pang, Katerina Goseva-Popstojanova, Maura McLaughlin. "Detection of Radio Pulsars in Single-pulse Searches Within and Across Surveys", Publications of the Astronomical Society of the Pacific, 132(1015), 2020.

3. Di Pang, Katerina Goseva-Popstojanova, Thomas Devine, Maura McLaughlin. "A Novel Single pulse Search Approach to Detection of Dispersed Radio Pulses Using Clustering and Supervised Machine Learning", Monthly Notices of the Royal Astronomical Society, 480(3):3302-3323, 2018.

4. Thomas R Devine, Katerina Goseva-Popstojanova, Di Pang. "Scalable Solutions for Automated Single Pulse Identification and Classification in Radio Astronomy", Proceedings of the 47th International Conference on Parallel Processing, 1-11, 2018. 


\section{Bibliography}

[1] S. Ackermann, K. Schawinski, C. Zhang, A. K. Weigel, and M. Dennis Turp, "Using transfer learning to detect galaxy mergers," Monthly Notices of the Royal Astronomical Society, vol. 479, no. 1, pp. 415-425, 2018.

[2] C. C. Aggarwal, Recommender Systems. Springer, 2016.

[3] R. A. Armstrong, "When to use the bonferroni correction," Ophthalmic and Physiological Optics, vol. 34, no. 5, pp. 502-508, 2014. [Online]. Available: https://onlinelibrary.wiley.com/doi/abs/10.1111/opo.12131

[4] M. Baak, R. Koopman, H. Snoek, and S. Klous, "A new correlation coefficient between categorical, ordinal and interval variables with Pearson characteristics," Computational Statistics and Data Analysis, vol. 152, 2020.

[5] S. D. Bates, M. Bailes, B. R. Barsdell, N. D. R. Bhat, M. Burgay, S. BurkeSpolaor, D. J. Champion, P. Coster, N. D'Amico, A. Jameson, S. Johnston, M. J. Keith, M. Kramer, L. Levin, A. Lyne, S. Milia, C. Ng, C. Nietner, A. Possenti, B. Stappers, D. Thornton, and W. van Straten, "The high time resolution universe pulsar survey - VI. An artificial neural network and timing of 75 pulsars," Monthly Notices of the Royal Astronomical Society, vol. 427, no. 2, pp. 1052-1065, 122012.

[6] S. Bethapudi and S. Desai, "Separation of pulsar signals from noise using supervised machine learning algorithms," Astronomy and Computing, vol. 23, pp. 15-26, 2018.

[7] H. Blumer, M. A. McLaughlin, J. Stewart, K. Williamson, D. R. Lorimer, S. A. Heatherly, J. K. Swiggum, R. S. Lynch, C. Zabriskie, N. Lewandowska, A. Roy, and S. Au, "The pulsar search collaboratory: Current status and future prospects," American Journal of Physics, vol. 88, no. 1, pp. 31-38, Jan. 2020.

[8] J. Boyles, R. S. Lynch, S. M. Ransom, I. H. Stairs, D. R. Lorimer, M. A. McLaughlin, J. W. T. Hessels, V. M. Kaspi, V. I. Kondratiev, A. Archibald, A. Berndsen, R. F. Cardoso, A. Cherry, C. R. Epstein, C. Karako-Argaman, C. A. McPhee, T. Pennucci, M. S. E. Roberts, K. Stovall, and J. van Leeuwen, "The green bank telescope $350 \mathrm{mhz}$ drift-scan survey. i. survey observations and the discovery of 13 pulsars," The Astrophysical Journal, vol. 763, no. 2, p. 80, jan 2013.

[9] L. Breiman, "Random forests," Machine Learning, vol. 45, no. 1, pp. 5-32, Oct 2001. 
[10] D. Bridge, M. H. Göker, L. McGinty, and B. Smyth, "Case-based recommender systems," The Knowledge Engineering Review, vol. 20, no. 3, pp. 315-320, 2006.

[11] S. Burke-Spolaor and M. Bailes, "The millisecond radio sky: Transients from a blind single-pulse search," Monthly Notices of the Royal Astronomical Society, vol. 402, no. 2, pp. 855-866, 2010.

[12] S. Burke-Spolaor, M. Bailes, R. Ekers, J.-P. Macquart, and F. C. III, "Radio bursts with extragalactic spectral characteristics show terrestrial origins," The Astrophysical Journal, vol. 727, no. 1, p. 18, dec 2010.

[13] J. Castro, R. M. Rodriguez, and M. J. Barranco, "Weighting of features in contentbased filtering with entropy and dependence measures," International Journal of Computational Intelligence Systems, vol. 7, no. 1, pp. 80-89, 2014.

[14] N. V. Chawla, K. W. Bowyer, L. O. Hall, and W. P. Kegelmeyer, "Smote: synthetic minority over-sampling technique," Journal of artificial intelligence research, vol. 16, no. 1, pp. 321-357, 2002.

[15] W. W. Cohen, "Fast effective rule induction," in Twelfth International Conference on Machine Learning. Morgan Kaufmann, 1995, pp. 115-123.

[16] J. J. Condon and S. M. Ransom, Essential Radio Astronomy. Princeton University Press, 2016.

[17] L. Connor and J. van Leeuwen, "Applying deep learning to fast radio burst classification," The Astronomical Journal, vol. 156, no. 6, p. 256, nov 2018.

[18] J. M. Cordes, P. C. C. Freire, D. R. Lorimer, F. Camilo, D. J. Champion, D. J. Nice, R. Ramachandran, J. W. T. Hessels, W. Vlemmings, J. van Leeuwen, S. M. Ransom, N. D. R. Bhat, Z. Arzoumanian, M. A. McLaughlin, V. M. Kaspi, L. Kasian, J. S. Deneva, B. Reid, S. Chatterjee, J. L. Han, D. C. Backer, I. H. Stairs, A. A. Deshpande, and C. Faucher-Giguere, "Arecibo pulsar survey using alfa. I. Survey strategy and first discoveries," The Astrophysical Journal, vol. 637, no. 1, pp. 446-455, 2006.

[19] J. M. Cordes and M. A. McLaughlin, "Searches for fast radio transients," The Astrophysical Journal, vol. 596, pp. 1142-1154, 2003.

[20] W. Dai, Q. Yang, G.-R. Xue, and Y. Yu, "Boosting for transfer learning," in Proceedings of the 24th international conference on Machine learning - ICML 'O\%. New York, NY: Association for Computing Machinery, 2007, pp. 193-200.

[21] S. Das, S. Datta, and B. B. Chaudhuri, "Handling data irregularities in classification: Foundations, trends, and future challenges," Pattern Recognition, vol. 81, pp. 674-693, 2018.

[22] J. Davis and M. Goadrich, "The relationship between precision-recall and ROC curves," in ICML '06: Proceedings of the 23rd international conference on Machine learning, W. Cohen and A. Moore, Eds., vol. 148. New York, NY: Association for Computing Machinery, 2006, pp. 233-240. 
[23] J. S. Deneva, J. M. Cordes, M. A. McLaughlin, D. J. Nice, D. R. Lorimer, F. Crawford, N. D. R. Bhat, F. Camilo, D. J. Champion, P. C. C. Freire, S. Edel, V. I. Kondratiev, J. W. T. Hessels, F. A. Jenet, L. Kasian, V. M. Kaspi, M. Kramer, P. Lazarus, S. M. Ransom, I. H. Stairs, B. W. Stappers, J. van Leeuwen, A. Brazier, A. Venkataraman, J. A. Zollweg, and S. Bogdanov, "Arecibo pulsar survey using alfa: Probing radio pulsar intermittency and transients," The Astrophysical Journal, vol. 703, no. 2, pp. 2259-2274, 2009.

[24] J. S. Deneva, K. Stovall, M. A. McLaughlin, M. Bagchi, S. D. Bates, P. C. C. Freire, J. G. Martinez, F. Jenet, and N. Garver-Daniels, "New discoveries from the Arecibo $327 \mathrm{MHz}$ drift pulsar survey radio transient search," The Astrophysical Journal, vol. 821, no. 1, p. 10, 2016.

[25] J. S. Deneva, K. Stovall, M. A. McLaughlin, S. D. Bates, P. C. Freire, J. G. Martinez, F. Jenet, and M. Bagchi, "Goals, strategies and first discoveries of ao327, the arecibo all-sky $327 \mathrm{MHz}$ drift pulsar survey," Astrophysical Journal, vol. 775, no. $1,2013$.

[26] T. R. Devine, K. Goseva-Popstojanova, and M. McLaughlin, "Detection of dispersed radio pulses: a machine learning approach to candidate identification and classification," Monthly Notices of the Royal Astronomical Society, vol. 459, pp. 1519-1532, Jun. 2016.

[27] T. R. Devine, K. Goseva-popstojanova, and D. Pang, "Scalable solutions for automated single pulse identification and classification in radio astronomy," in ICPP 2018 Proceedings of the 47 th International Conference on Parallel Processing. New York, NY: Association for Computing Machinery, 2018.

[28] T. R. Devine, "Searching for needles in the cosmic haystack," Ph.D. dissertation, West Virginia University, 2020.

[29] P. Domingos, "A few useful things to know about machine learning," Communications of the ACM, vol. 55, no. 10, p. 78, 2012.

[30] R. P. Eatough, N. Molkenthin, M. Kramer, A. Noutsos, M. J. Keith, B. W. Stappers, and A. G. Lyne, "Selection of radio pulsar candidates using artificial neural networks," Monthly Notices of the Royal Astronomical Society, vol. 407, no. 4, pp. 2443-2450, 2010.

[31] A. Estabrooks, T. Jo, and N. Japkowicz, "A multiple resampling method for learning from imbalanced data sets," Computational Intelligence, vol. 20, pp. 18-36, 2004 .

[32] M. Ester, H. P. Kriegel, J. Sander, and X. Xu, "A density-based algorithm for discovering clusters in large spatial databases with noise," Second International Conference on Knowledge Discovery and Data Mining, pp. 226-231, 1996.

[33] A. J. Faulkner, I. H. Stairs, M. Kramer, A. G. Lyne, G. Hobbs, A. Possenti, D. R. Lorimer, R. N. Manchester, M. A. McLaughlin, N. D'Amico, F. Camilo, and M. Burgay, "The Parkes multibeam pulsar survey - V. Finding binary and millisecond pulsars," Monthly Notices of the Royal Astronomical Society, vol. 355, no. 1, pp. 147-158, 112004. 
[34] G. Foster, A. Karastergiou, G. Golpayegani, M. Surnis, D. R. Lorimer, J. Chennamangalam, M. McLaughlin, W. Armour, J. Cobb, D. H. E. MacMahon, X. Pei, K. Rajwade, A. P. V. Siemion, D. Werthimer, and C. J. Williams, "ALFABURST: a commensal search for fast radio bursts with Arecibo," Monthly Notices of the Royal Astronomical Society, vol. 474, no. 3, pp. 3847-3856, 112017.

[35] E. Frank, M. A. Hall, and I. H. Witten, The WEKA workbench. Online appendix for "Data Mining: Practical Machine Learning Tools and Techniques". Morgan Kaufmann, Fourth Edition, 2016.

[36] E. Frank and I. H. Witten, "Generating accurate rule sets without global optimization," in Fifteenth International Conference on Machine Learning, J. Shavlik, Ed. Morgan Kaufmann, 1998, pp. 144-151.

[37] Y. Freund and R. E. Schapire, "A decision-theoretic generalization of on-line learning and an application to boosting," J. Comput. Syst. Sci., vol. 55, no. 1, pp. 119-139, Aug. 1997.

[38] V. García, J. S. Sánchez, H. J. Ochoa Domínguez, and L. Cleofas-Sánchez, "Dissimilarity-based learning from imbalanced data with small disjuncts and noise," in Pattern Recognition and Image Analysis, R. Paredes, J. S. Cardoso, and X. M. Pardo, Eds. Cham: Springer International Publishing, 2015, pp. 370-378.

[39] M. Ghifary, W. B. Kleijn, and M. Zhang, "Domain adaptive neural networks for object recognition," in PRICAI 2014: Trends in Artificial Intelligence, 2014, pp. 898-904.

[40] D. Gómez and A. Rojas, "An empirical overview of the no free lunch theorem and its effect on real-world machine learning classification," Neural Computation, vol. 28, no. 1, pp. 216-228, 2016.

[41] I. Goodfellow, Y. Bengio, and A. Courville, Deep Learning. MIT Press, 2016, http://www.deeplearningbook.org.

[42] R. Gopalan, Ruonan Li, and R. Chellappa, "Domain adaptation for object recognition: An unsupervised approach," in 2011 International Conference on Computer Vision, Nov 2011, pp. 999-1006.

[43] P. Guo, F. Duan, P. Wang, Y. Yao, Q. Yin, X. Xin, D. Li, L. Qian, S. Wang, Z. Pan, and L. Zhang, "Pulsar candidate classification using generative adversary networks," Mnras, vol. 5439, pp. 5424-5439, 2019.

[44] K. D. Gupta, R. Pampana, R. Vilalta, E. E. Ishida, and R. S. De Souza, "Automated supernova ia classification using adaptive learning techniques," in 2016 IEEE Symposium Series on Computational Intelligence (SSCI). Piscataway, NJ: IEEE, 2016, pp. 1-8.

[45] J. Han, M. Kamber, and J. Pei, Data Mining: Concepts and Techniques, 3rd ed. San Francisco, CA, USA: Morgan Kaufmann Publishers Inc., 2011.

[46] M. Hassaballah and A. I. Awad, Deep Learning in Computer Vision: Principles and Applications, 1st ed. Boca Raton, FL, USA: CRC Press, 2020. 
[47] H. He and Y. Ma, Introduction. John Wiley \& Sons, Ltd, 2013, pp. 1-12.

[48] A. Hewish, S. J. Bell, J. D. H. Pilkington, P. F. Scott, and R. A. Collins, "Observation of a rapidly pulsating radio source," Nature, vol. 217, pp. 709-713, Feb. 1968.

[49] D. Kar, S. Chakraborti, and B. Ravindran, "Feature weighting and confidence based prediction for case based reasoning systems," in Case-Based Reasoning Research and Development, B. D. Agudo and I. Watson, Eds. Berlin, Heidelberg: Springer Berlin Heidelberg, 2012, pp. 211-225.

[50] C. Karako-Argaman, V. M. Kaspi, R. S. Lynch, J. W. T. Hessels, V. I. Kondratiev, M. A. McLaughlin, S. M. Ransom, A. M. Archibald, J. Boyles, F. A. Jenet, D. L. Kaplan, L. Levin, D. R. Lorimer, E. C. Madsen, M. S. E. Roberts, X. Siemens, I. H. Stairs, K. Stovall, J. K. Swiggum, and J. van Leeuwen, "Discovery and followup of rotating radio transients with the Green Bank and Lofar telescopes," The Astrophysical Journal, vol. 809, no. 1, p. 67, 2015.

[51] E. Kasuya, "On the use of $\mathrm{r}$ and $\mathrm{r}$ squared in correlation and regression," Ecological Research, vol. 34, no. 1, pp. 235-236, 2019. [Online]. Available: https://esj-journals.onlinelibrary.wiley.com/doi/abs/10.1111/1440-1703.1011

[52] E. F. Keane, D. A. Ludovici, R. P. Eatough, M. Kramer, A. G. Lyne, M. A. McLaughlin, and B. W. Stappers, "Further searches for rotating radio transients in the parkes multi-beam pulsar survey," Monthly Notices of the Royal Astronomical Society, vol. 401, no. 2, pp. 1057-1068, 2010.

[53] M. J. Keith, R. P. Eatough, A. G. Lyne, M. Kramer, A. Possenti, F. Camilo, and R. N. Manchester, "Discovery of 28 pulsars using new techniques for sorting pulsar candidates," Monthly Notices of the Royal Astronomical Society, vol. 395, no. 2, pp. 837-846, 2009.

[54] A. Krizhevsky, I. Sutskever, and G. E. Hinton, "Imagenet classification with deep convolutional neural networks," in Advances in Neural Information Processing Systems, F. Pereira, C. J. C. Burges, L. Bottou, and K. Q. Weinberger, Eds., vol. 25. Curran Associates, Inc., 2012.

[55] L. La, Q. Guo, Q. Cao, and Y. Wang, "Transfer learning with reasonable boosting strategy," Neural Computing and Applications, vol. 24, no. 3, pp. 807-816, Mar 2014.

[56] M. Long, J. Wang, G. Ding, J. Sun, and P. S. Yu, "Transfer joint matching for unsupervised domain adaptation," in 2014 IEEE Conference on Computer Vision and Pattern Recognition, June 2014, pp. 1410-1417.

[57] M. Long, Y. Cao, J. Wang, and M. Jordan, "Learning transferable features with deep adaptation networks," in Proceedings of the 32nd International Conference on Machine Learning, ser. Proceedings of Machine Learning Research, F. Bach and D. Blei, Eds., vol. 37. Lille, France: PMLR, 07-09 Jul 2015, pp. 97-105. 
[58] D. R. Lorimer, M. Bailes, M. A. McLaughlin, D. J. Narkevic, and F. Crawford, "A bright millisecond radio burst of extragalactic origin." Science, vol. 318, no. 5851, pp. $777-80,2007$.

[59] D. R. Lorimer and M. Kramer, Handbook of Pulsar Astronomy. Cambridge University Press, Cambridge, Dec. 2004.

[60] R. Lynch, "From the telescope to the collaboratory: Processing gbt data," Tutorial Materials, pp. 1-12, 2013.

[61] R. S. Lynch, J. Boyles, S. M. Ransom, I. H. Stairs, D. R. Lorimer, M. A. McLaughlin, J. W. T. Hessels, V. M. Kaspi, V. I. Kondratiev, A. M. Archibald, A. Berndsen, R. F. Cardoso, A. Cherry, C. R. Epstein, C. Karako-Argaman, C. A. McPhee, T. Pennucci, M. S. E. Roberts, K. Stovall, and J. van Leeuwen, "The green bank telescope $350 \mathrm{mhz}$ drift-scan survey ii: Data analysis and the timing of the 10 new pulsars, including a relativistic binary," The Astrophysical Journal, vol. 763, no. 2, p. 81, jan 2013.

[62] R. J. Lyon, J. M. Brooke, J. D. Knowles, and B. W. Stappers, "A study on classification in imbalanced and partially-labelled data streams," in 2013 IEEE International Conference on Systems, Man, and Cybernetics. Piscataway, NJ: IEEE, 2013, pp. 1506-1511.

[63] R. J. Lyon, B. W. Stappers, S. Cooper, J. M. Brooke, and J. D. Knowles, "Fifty years of pulsar candidate selection: from simple filters to a new principled real-time classification approach," Monthly Notices of the Royal Astronomical Society, vol. 459, no. 1, pp. 1104-1123, 042016.

[64] R. J. Lyon, "Why are pulsars hard to find?" Ph.D. dissertation, The University of Manchester, 2016.

[65] R. N. Manchester, G. B. Hobbs, A. Teoh, and M. Hobbs, "The Australia Telescope National Facility Pulsar Catalogue," The Astrophysical Journal, vol. 129, pp. 19932006, Apr. 2005.

[66] R. Manchester, A. Lyne, F. Camilo, J. Bell, V. Kaspi, N. D’Amico, N. McKay, F. Crawford, I. Stairs, A. Possenti, M. Kramer, and D. Sheppard, "The Parkes multi-beam pulsar survey - I. Observing and data analysis systems, discovery and timing of 100 pulsars," Monthly Notices of the Royal Astronomical Society, vol. 328, no. 1, pp. 17-35, 112001.

[67] A. J. Masino, M. C. Harris, D. Forsyth, S. Ostapenko, L. Srinivasan, C. P. Bonafide, F. Balamuth, M. Schmatz, and R. W. Grundmeier, "Machine learning models for early sepsis recognition in the neonatal intensive care unit using readily available electronic health record data," PLOS ONE, vol. 14, no. 2, pp. 1-23, 022019.

[68] M. A. McLaughlin, A. G. Lyne, D. R. Lorimer, M. Kramer, A. J. Faulkner, R. N. Manchester, J. M. Cordes, F. Camilo, A. Possenti, I. H. Stairs, G. Hobbs, N. D'Amico, M. Burgay, and J. T. O'Brien, "Transient radio bursts from rotating neutron stars," Nature, vol. 439, no. 7078, pp. 817-820, 2006. 
[69] R. V. Meteren and M. V. Someren, "Using content-based filtering for recommendation," ECML/MLNET Workshop on Machine Learning and the New Information Age, pp. 47-56, 2000.

[70] D. Michilli, J. W. Hessels, R. J. Lyon, C. M. Tan, C. Bassa, S. Cooper, V. I. Kondratiev, S. Sanidas, B. W. Stappers, and J. van Leeuwen, "Single-pulse classifier for the LOFAR tied-array all-sky survey," Monthly Notices of the Royal Astronomical Society, vol. 480, no. 3, pp. 3457-3467, 2018.

[71] P. Mignone, G. Pio, S. Džeroski, and M. Ceci, "Multi-task learning for the simultaneous reconstruction of the human and mouse gene regulatory networks," Scientific Reports, vol. 10, no. 1, dec 2020.

[72] T. M. Mitchell, Machine Learning, 1st ed. New York, NY, USA: McGraw-Hill, Inc., 1997.

[73] V. Morello, E. D. Barr, M. Bailes, C. M. Flynn, E. F. Keane, and W. van Straten, "SPINN: A straightforward machine learning solution to the pulsar candidate selection problem," Monthly Notices of the Royal Astronomical Society, vol. 443, no. 2, pp. 1651-1662, 2014.

[74] K. P. Murphy, Machine Learning: A Probabilistic Perspective. The MIT Press, 2012.

[75] N. Nikolaou, N. Edakunni, M. Kull, P. Flach, and G. Brown, "Cost-sensitive boosting algorithms: Do we really need them?" Machine Learning, vol. 104, no. 2, pp. 359-384, Sep 2016.

[76] A. Ouaknine, "Review of deep learning algorithms for object detection." Feburary 2018, [Online]. [Online]. Available: https://medium.com/zylapp/ review-of-deep-learning-algorithms-for-object-detection-c1f3d437b852

[77] S. J. Pan, I. W. Tsang, J. T. Kwok, and Q. Yang, "Domain adaptation via transfer component analysis," IEEE Transactions on Neural Networks, vol. 22, no. 2, pp. 199-210, Feb 2011.

[78] S. J. Pan and Q. Yang, "A survey on transfer learning," IEEE Transactions on Knowledge and Data Engineering, vol. 22, no. 10, pp. 1345-1359, 2010.

[79] D. Pang, K. Goseva-Popstojanova, T. Devine, and M. McLaughlin, "A novel singlepulse search approach to detection of dispersed radio pulses using clustering and supervised machine learning," Monthly Notices of the Royal Astronomical Society, vol. 480, no. 3, pp. 3302-3323, 082018.

[80] D. Pang, K. Goseva-popstojanova, and M. Mclaughlin, "Detection of radio pulsars in single-pulse searches within and across surveys," Publications of the Astronomical Society of the Pacific, vol. 132, no. 1015, p. 94502, 2020.

[81] D. Pang, K. Goseva-Popstojanova, and M. McLaughlin, "Hybrid recommender system for detection of rare cases applied to pulsar candidate selection," in 2021 IEEE 8th International Conference on Data Science and Advanced Analytics (DSAA), 2021, pp. 1-10. 
[82] M. J. Pazzani, "A framework for collaborative, content-based and demographic filtering," Artificial Intelligence Review, vol. 13, no. 5, pp. 393-408, 1999.

[83] F. Pedregosa, G. Varoquaux, A. Gramfort, V. Michel, B. Thirion, O. Grisel, M. Blondel, P. Prettenhofer, R. Weiss, V. Dubourg, J. Vanderplas, A. Passos, D. Cournapeau, M. Brucher, M. Perrot, and É. Duchesnay, "Scikit-learn: machine learning in Python," Journal of Machine Learning Research, vol. 12, pp. 2825$2830,2011$.

[84] E. Petroff, E. F. Keane, E. D. Barr, J. E. Reynolds, J. Sarkissian, P. G. Edwards, J. Stevens, C. Brem, A. Jameson, S. Burke-Spolaor, S. Johnston, N. D. R. Bhat, P. C. S. Kudale, and S. Bhandari, "Identifying the source of perytons at the Parkes radio telescope," Monthly Notices of the Royal Astronomical Society, vol. 451, no. 4, pp. 3933-3940, 062015.

[85] H. N. A. Pham and E. Triantaphyllou, The impact of overfitting and overgeneralization on the classification accuracy in data mining. Boston, MA: Springer US, 2008, pp. 391-431.

[86] J. C. Platt, "Advances in kernel methods," B. Schölkopf, C. J. C. Burges, and A. J. Smola, Eds. Cambridge, MA, USA: MIT Press, 1999, ch. Fast training of support vector machines using sequential minimal optimization, pp. 185-208.

[87] J. R. Quinlan, C4.5: Programs for Machine Learning. San Francisco, CA, USA: Morgan Kaufmann Publishers Inc., 1993.

[88] N. N. R. Ranga Suri, N. Murty M, and G. Athithan, Computational preliminaries. Cham: Springer International Publishing, 2019, pp. 53-68.

[89] S. M. Ransom, "New search techniques for binary pulsars," Ph.D. dissertation, Harvard Univ., 2001.

[90] E. M. Rasmussen, "Clustering algorithms," in Information Retrieval: Data Structures and Algorithms, W. B. Frakes and R. A. Baeza-Yates, Eds. Prentice-Hall, 1992, pp. 419-442.

[91] F. Ricci, L. Rokach, and B. Shapira, Recommender systems handbook. Springer, 2015.

[92] R. Rosen, S. Heatherly, M. A. McLaughlin, R. Lynch, V. I. Kondratiev, J. R. Boyles, M. Wilson, D. R. Lorimer, and S. Ransom, "The pulsar search collaboratory," Astronomy Education Review, vol. 9, no. 1, p. 010106, Jan. 2010.

[93] R. Rosen, J. Swiggum, M. A. McLaughlin, D. R. Lorimer, M. Yun, S. A. Heatherly, J. Boyles, R. Lynch, V. I. Kondratiev, S. Scoles, S. M. Ransom, M. L. Moniot, A. Cottrill, M. Weaver, A. Snider, C. Thompson, M. Raycraft, J. Dudenhoefer, L. Allphin, J. Thorley, B. Meadows, G. Marchiny, A. Liska, A. M. O'Dwyer, B. Butler, S. Bloxton, H. Mabry, H. Abate, J. Boothe, S. Pritt, J. Alberth, A. Green, R. J. Crowley, A. Agee, S. Nagley, N. Sargent, E. Hinson, K. Smith, R. McNeely, H. Quigley, A. Pennington, S. Chen, T. Maynard, L. Loope, N. Bielski, J. R. McGough, J. C. Gural, S. Colvin, S. Tso, Z. Ewen, M. Zhang, N. Ciccarella, 
B. Bukowski, C. B. Novotny, J. Gore, K. Sarver, S. Johnson, H. Cunningham, D. Collins, D. Gardner, A. Monteleone, J. Hall, R. Schweinhagen, J. Ayers, S. Jay, B. Uosseph, D. Dunkum, J. Pal, S. Dydiw, M. Sterling, and E. Phan, "The pulsar search collaboratory: Discovery and timing of five new pulsars," The Astrophysical Journal, vol. 768, no. 1, p. 85, apr 2013.

[94] B. C. Ross, "Mutual information between discrete and continuous data sets," PLoS ONE, vol. 9, no. 2, 2014.

[95] P. J. Rousseeuw and M. Hubert, "Robust statistics for outlier detection," Wiley Interdisciplinary Reviews: Data Mining and Knowledge Discovery, vol. 1, no. 1, pp. 73-79, 2011.

[96] T. Ryan Hoens and N. V. Chawla, Imbalanced datasets: From sampling to classifiers. John Wiley \& Sons, Ltd, 2013, pp. 43-59.

[97] I. Schwab, A. Kobsa, and I. Koychev, "Learning user interests through positive examples using content analysis and collaborative filtering," User Modeling and User-adapted Interaction - UMUAI, vol. 14, no. 5, pp. 469-475, 2004.

[98] F. Sultana, A. Sufian, and P. Dutta, A review of object detection models based on convolutional neural network. Singapore: Springer Singapore, 2020, pp. 1-16.

[99] J. K. Swiggum, D. R. Lorimer, M. A. McLaughlin, S. D. Bates, D. J. Champion, S. M. Ransom, P. Lazarus, A. Brazier, J. W. T. Hessels, D. J. Nice, J. Ellis, T. R. Senty, B. Allen, N. D. R. Bhat, S. Bogdanov, F. Camilo, S. Chatterjee, J. M. Cordes, F. Crawford, J. S. Deneva, P. C. C. Freire, F. A. Jenet, C. KarakoArgaman, V. M. Kaspi, B. Knispel, K. J. Lee, J. van Leeuwen, R. Lynch, A. G. Lyne, P. Scholz, X. Siemens, I. H. Stairs, B. W. Stappers, K. Stovall, A. Venkataraman, and W. W. Zhu, "Arecibo pulsar survey using alfa. III. Precursor survey and population synthesis," The Astrophysical Journal, vol. 787, no. 2, p. 137, 2014.

[100] C. Tan, F. Sun, T. Kong, W. Zhang, C. Yang, and C. Liu, "A survey on deep transfer learning," in Artificial Neural Networks and Machine Learning - ICANN 2018, V. Kůrková, Y. Manolopoulos, B. Hammer, L. Iliadis, and I. Maglogiannis, Eds. Cham: Springer International Publishing, 2018, pp. 270-279.

[101] E. Tzeng, J. Hoffman, N. Zhang, K. Saenko, and T. Darrell, "Deep domain confusion: Maximizing for domain invariance," Computing Research Repository, 2014.

[102] S. Uguroglu and J. Carbonell, "Feature selection for transfer learning," in $\mathrm{Ma}$ chine Learning and Knowledge Discovery in Databases, D. Gunopulos, T. Hofmann, D. Malerba, and M. Vazirgiannis, Eds. Berlin, Heidelberg: Springer Berlin Heidelberg, 2011, pp. 430-442.

[103] A. van den Bosch, T. Weijters, H. J. V. D. Herik, and W. Daelemans, "When small disjuncts abound, try lazy learning: A case study," in BENELEARN-97: proceedings of the seventh Belgian-Dutch Conference on Machine Learning. Tilburg, Netherlands: Tilburg University, 1997, pp. 109-118. 
[104] K. L. Wagstaff, B. Tang, D. R. Thompson, S. Khudikyan, J. Wyngaard, A. T. Deller, D. Palaniswamy, S. J. Tingay, and R. B. Wayth, "A machine learning classifier for fast radio burst detection at the VLBA," Publications of the Astronomical Society of the Pacific, vol. 128, no. 966, p. 084503, jun 2016.

[105] G. Weiss, "Mining with rarity: A unifying framework," SIGKDD Explorations Newsletter, vol. 6, no. 1, pp. 7-19, Jun. 2004.

[106] G. M. Weiss, Foundations of imbalanced learning. John Wiley \& Sons, Ltd, 2013, ch. 2 , pp. 13-41.

[107] G. Weiss, "Learning with rare cases and small disjuncts," in Machine Learning Proceedings 1995, A. Prieditis and S. Russell, Eds. San Francisco, CA: Morgan Kaufmann, 1995, pp. $558-565$.

[108] K. Weiss, T. M. Khoshgoftaar, and D. D. Wang, "A survey of transfer learning," Journal of Big Data, vol. 3, no. 1, 2016.

[109] I. H. Witten, E. Frank, and M. A. Hall, Data Mining: Practical Machine Learning Tools and Techniques, 3rd ed. San Francisco, CA, USA: Morgan Kaufmann Publishers Inc., 2011.

[110] D. H. Wolpert, "The lack of a priori distinctions between learning algorithms," Neural Computation, vol. 8, no. 7, pp. 1341-1390, 1996.

[111] J. Yosinski, J. Clune, Y. Bengio, and H. Lipson, "How transferable are features in deep neural networks?" in Advances in Neural Information Processing Systems 27. Red Hook, NY: Curran Associates, Inc., 2014, pp. 3320-3328.

[112] M. D. Zeiler and R. Fergus, "Visualizing and understanding convolutional networks," in Computer Vision - ECCV 2014, D. Fleet, T. Pajdla, B. Schiele, and T. Tuytelaars, Eds. Cham: Springer International Publishing, 2014, pp. 818-833.

[113] Z.-H. Zhou, Ensemble learning. Singapore: Springer Singapore, 2021, pp. 181-210.

[114] W. W. Zhu, A. Berndsen, E. C. Madsen, M. Tan, I. H. Stairs, A. Brazier, P. Lazarus, R. Lynch, P. Scholz, K. Stovall, S. M. Ransom, S. Banaszak, C. M. Biwer, S. Cohen, L. P. Dartez, J. Flanigan, G. Lunsford, J. G. Martinez, A. Mata, M. Rohr, A. Walker, B. Allen, N. D. R. Bhat, S. Bogdanov, F. Camilo, S. Chatterjee, J. M. Cordes, F. Crawford, J. S. Deneva, G. Desvignes, R. D. Ferdman, P. C. C. Freire, J. W. T. Hessels, F. A. Jenet, D. L. Kaplan, V. M. Kaspi, B. Knispel, K. J. Lee, J. van Leeuwen, A. G. Lyne, M. A. McLaughlin, X. Siemens, L. G. Spitler, and A. Venkataraman, "Searching for pulsars using image pattern recognition," The Astrophysical Journal, vol. 781, no. 2, p. 117, 2014. 